Н. Н. Веричев, С. Н. Веричев, С. И. Герасимов, В. И. Ерофеев

\title{
ХАОС, СИНХРОНИЗАЦИЯ И СТРУКТУРЫ В ДИНАМИКЕ РОТАТОРОВ
}

Библиотека журнала "Вестник научно-техническо го развития"

The library of the Journal "Bulletin of Science and Technical Development" (http://www.vntr.ru)

Представлено авторами

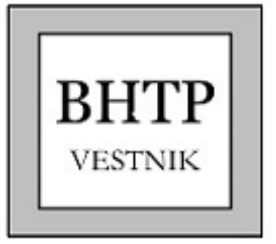

MOCKBA

2017 
ФГУП «Российский федеральный ядерный центр Всероссийский научно-исследовательский институт экспериментальной физики»

\author{
Н. Н. Веричев, С. Н. Веричев, \\ С. И. Герасимов, В. И. Ерофеев
}

\title{
ХАОС, СИНХРОНИЗАЦИЯ И СТРУКТУРЫ В ДИНАМИКЕ РОТАТОРОВ
}


ББК 22.161.6

X 19

\author{
Веричев, Н. Н., Веричев, С. Н., Герасимов, С. И., Ерофе- \\ Х 19 ев, В. И. Хаос, синхронизация и структуры в динамике ротато- \\ ров / Н. Н. Веричев, С. Н. Веричев, С. И. Герасимов, В. И. Ерофеев. - \\ Саров: РФЯЦ-ВНИИЭФ, 2016. - 267 с., ил.
}

ISBN 978-5-9515-0324-4

Монография посвящена динамике систем с цилиндрическим фазовым пространством. Данный класс моделей охватывает механические, квантово-механические, радиотехнические и многие другие системы из различных областей естествознания и технических приложений. Рассматриваются автономные и неавтономные системы с одной, полутора, двумя и более степенями свободы. Исследования и изложение материала проводятся в традициях школы нелинейных колебаний академика А. А. Андронова: в контексте фазового пространства моделей с привлечением методов качественной теории дифференциальных уравнений, теории бифуркаций и качественно-численных методов. Исследуются качественные картины характеристик вращения ротаторов и резонансные характеристики колебательных систем. Показана неопределенность и нестабильность этих характеристик в областях существования странных аттракторов, среди которых аттракторы Лоренца, Фейгенбаума, Шильникова и др. Рассматриваются кластерные структуры в однородных и упорядоченно неоднородных решетках динамических систем. Дана классификация кластерных структур и доказана ограниченность числа их типов для цепочки и кольца. Рассматриваются вопросы устойчивости кластерных структур.

Издание предназначено для студентов вузов и аспирантов, а также специалистов, работающих в области машиностроения.

УДК 517.938

ББК 22.161.6 


\section{ОГЛАВЛЕНИЕ}

Предисловие .......................................... 5

ГЛава 1. АВТОНОМНЫЕ И НЕАВТОНОМНЫЕ

СИСТЕМЫ С ОДНОЙ СТЕПЕНЬЮ СВОБОДЫ ............ 8

1.1. Динамика автономного ротатора и физические системы ......................................... 8

1.2. Синхронизация и хаотические вращения

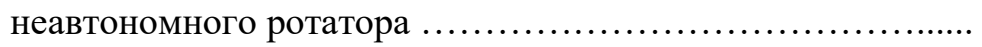

Глава 2. АВТОНОМНЫЕ И НЕАВТОНОМНЫЕ СИСТЕМЫ С ПОЛУТОРА СТЕПЕНЯМИ СВОБОДЫ ..... 39

2.1. Динамика ротатора с апериодической нагрузкой ........ 39

2.2. Синхронизация и динамический хаос в системе неавтономного ротатора с апериодической нагрузкой ...... 51

\section{Глава 3. АВТОНОМНЫЕ СИСТЕМЫ С ДВУМЯ} СТЕПЕНЯМИ СВОБОДЫ ................................... 69

3.1. Динамика ротатора с колебательной нагрузкой ......... 69

3.2. Хаотическая динамика простого вибрационного механизма ............................................. 78

3.3. Динамика связанных ротаторов ...................... 94

Глава 4. КОЛЕБАНИЯ ВАЛОВ ............................... 107

4.1. Нелинейный резонанс изгибных колебаний гибкого ротора в системе с источником возбуждения ограниченной мощности ................................ 107

4.2. Гашение изгибных колебаний вала модуляцией частоты вращения двигателя .............................. 121

4.3. Хаотические крутильные колебания вала в системе с источником возбуждения ограниченной мощности ......... 131

\section{Глава 5. СИНХРОНИЗАЦИЯ В ОДНОРОДНЫХ}

5.1. Синхронизация в решетках динамических систем.

Общие сведения 
5.2. Синхронизация вращений в цепочке

и кольце диффузионно-связанных автономных

и неавтономных ротаторов ............................. 161

5.3. Регулярная и хаотическая синхронизация

в однородной цепочке динамических систем

«ротатор - осциллятор» ............................. 170

ГЛава 6. ФИЗИКА, СУЩЕСТВОВАНИЕ, СИНТЕЗ

И УСТОЙЧИВОСТЬ КЛАСТЕРНЫХ СТРУКТУР ......... 178

6.1. Физика кластерных структур ...................... 179

6.2. Синтез и общие свойства схем кластерных структур ..... 195

6.3. К-осцилляторы цепочки и полнота типов

ее кластерных структур .............................. 204

6.4. К-осцилляторы кольца и синтез

кластерных структур ............................... 218

6.5. Устойчивость кластерных структур ................. 232

Приложение І. Алгоритмы преобразования систем

связанных ротаторов к стандартной форме ................ 244

Приложение II. Вычисление собственных

значений матриц .................................... 257

Список литературы ..................................... 260 


\section{ПРЕДИСЛОВИЕ}

Системы физических маятников $[1,2]$ и системы сверхпроводящих переходов [3-5], системы связанных электрических машин [6] и вибрационные механизмы [7-9], системы фазовой синхронизации $[10,11]$ - далеко не полный набор систем из различных областей естествознания и технических приложений. Все они обладают общим свойством: некоторые физические переменные, определяющие динамическое состояние этих систем, являются пространственно-периодическими, иначе говоря, угловыми, фазовыми переменными. Наконец, главное: динамика названных систем описывается сходственными математическими моделями, заданными в цилиндрическом фазовом пространстве, - динамическими системами связанных ротаторов вида

$$
\begin{gathered}
I \ddot{\varphi}_{k}+\delta_{k}\left(1+F_{1 k}\left(\varphi_{k}\right)\right) \dot{\varphi}_{k}+\sigma_{k} F_{2 k}\left(\varphi_{k}\right)=\gamma_{k}+F_{k}(\boldsymbol{\varphi}, \dot{\boldsymbol{\varphi}}, \psi, z), \\
\dot{\mathbf{z}}=\mathbf{A z}+\mathbf{Z}(\mathbf{z}, \boldsymbol{\varphi}, \dot{\boldsymbol{\varphi}}, \psi), \\
\dot{\psi}=\omega_{0} .
\end{gathered}
$$

Здесь $k=\overline{1, n} ; \mathbf{A}-(m \times m)$ постоянная устойчивая матрица; $I, \delta_{k}$, $\sigma_{k}, \gamma_{k}, \omega_{0}$ - постоянные, безразмерные положительные параметры. Переменные $\varphi, \psi$ являются фазовыми (угловыми), причем $\varphi_{k}=\varphi_{k}+2 \pi(\bmod 2 \pi), \quad \psi=\psi+2 \pi(\bmod 2 \pi), \quad z \in R^{m}$. Дифференцирование ведется по безразмерному времени $\tau$. Функции $F_{1 k}\left(\varphi_{k}\right)$, $F_{2 k}\left(\varphi_{k}\right)$ с нулевым средним $\left\langle F_{1 k}\left(\varphi_{k}\right)\right\rangle_{\varphi_{k}}=0,\left\langle F_{2 k}\left(\varphi_{k}\right)\right\rangle_{\varphi_{k}}=0$, а также функции связей $F_{k}(\boldsymbol{\varphi}, \dot{\boldsymbol{\varphi}}, \psi, z), \mathbf{Z}(\boldsymbol{\varphi}, \dot{\boldsymbol{\varphi}}, \psi)$ являются периодическими по фазовым переменным. Система (П1) задана в цилиндрическом фазовом пространстве $G(\boldsymbol{\varphi}, \dot{\boldsymbol{\varphi}}, \psi, z)==T^{n+1} \times R^{n+m}$.

Единство математических моделей позволяет рассматривать не отдельные физические системы, а конкретные модели из класса (П1) как самостоятельные математические объекты с последующей интерпретацией результатов на различные физические 
сис-темы. С другой стороны, трансляция динамики моделей на их фазовые пространства позволяет не только адекватно описывать их нелинейные свойства, но и использовать весь арсенал методов качественной теории дифференциальных уравнений, теории бифуркаций и качественно-численных методов исследования. В данном контексте проводятся исследованиядинамики систем класса (П1) и дается изложение материала. Эта идея, ставшая методом современной науки, принадлежит А. А. Андронову, воплотившему еe в середине прошлого века в книге «Теория колебаний». Много лет спустя, по ставшей правилом случайности, она вернулась в отечественную науку из-за рубежа в виде «нового открытия» с нелепым названием «синергетика».

Монография состоит из шести глав и построена по принципу «от простого к сложному». В первой главе дан обзор свойств модели с одной степенью свободы и описываемых этой моделью физических систем. Вводятся основные понятия, конкретизируется предмет исследования и дается апробация методов.

Вторая глава посвящена динамике систем с полутора степенями свободы. Этими моделями описывается работа некоторых приборов сверхпроводниковой электроники, электродвигателей, работающих на разбалансированную апериодическую нагрузку, и других систем. Исследованы странные аттракторы, приводящие к хаотизации вращений ротаторов.

В третьей главе исследуются динамические свойства моделей с двумя степенями свободы. Представлены качественные картины характеристик вращения ротаторов, а также резонансных характеристик колебательных систем, выступающих в качестве нагрузок. Указывается на существование областей нестабильности этих характеристик, обусловленных странными аттракторами Лоренца и Фейгенбаума. Дается интерпретация результатов на физические системы.

Четвертая глава посвящена колебаниям валов, представляемых в рамках моделей из класса (П1). Показано, что кроме классического эффекта Зоммерфельда типичной стороной динамики валов является хаотизация крутильных колебаний. Изложен метод гашения изгибных колебаний валов путем управления частотой вращения исполнительного механизма.

В пятой главе рассматриваются вопросы синхронизации в решетках динамических систем, представляемых цепочкой, двумерной 
и трехмерной решеткой, а также кольцом идентичных связанных систем. Представлены условия локальной и глобальной устойчивости изохронной синхронизации.

Шестая глава посвящена кластерной динамике цепочки и кольца динамических систем. Вводятся необходимые понятия и определения, а также набор эквивалентных преобразований и правила синтеза схем кластерных структур. Дается классификация кластерных структур, доказываются теоремы о числе типов кластерных структур в цепочке и кольце. Приводятся результаты исследования устойчивости кластерных структур в решетках данного вида.

Работа выполнена при поддержке Российского научного фонда, проект № 14-19-01637. 


\section{Глава 1}

\section{АВТОНОМНЫЕ И НЕАВТОНОМНЫЕ СИСТЕМЫ С ОДНОЙ СТЕПЕНЬЮ СВОБОДЫ}

\section{1. Динамика автономного ротатора и физические системы}

Простейшей моделью из класса (П1) является автономный ротатор - дифференциальное уравнение вида

$$
I \ddot{\varphi}+\left(1+F_{1}(\varphi)\right) \dot{\varphi}+F_{2}(\varphi)=\gamma .
$$

Динамические свойства этого уравнения с различными выражениями нелинейных функций исследовались в многочисленных работах и достаточно хорошо известны $[1,2,12$ и др.]. Приведем их для случая $F_{1}(\varphi)=\varepsilon \cos \varphi, F_{2}(\varphi)=\sin \varphi,|\varepsilon| \leq 1$.

На рис. 1.1 показаны бифуркационная диаграмма (рис. 1.1,a) и фазовые портреты уравнения (1.1) на развертке фазового цилиндpa $(\varphi, \dot{\varphi})$ для каждой из областей параметров (все точки фазового портрета на левой и правой границах развертки отождествляются!). На бифуркационной диаграмме: $\lambda=I^{-1 / 2}, \lambda^{*}(\gamma)$ - бифуркационная кривая (Трикоми) петли сепаратрисы седла (седло-узла при $\lambda>\lambda_{0}$ ), при пересечении которой слева направо от петли рождается предельный цикл, а в обратном направлении цикл «влипает» в петлю. Бифуркационная линия $\gamma=1$ соответствует слиянию состояний равновесия с последующим их исчезновением, если пересечение прямой происходит слева направо, или рождению - при обратном ходе параметра.

На фазовых портретах (рис. 1.1,б-г): $O_{1}$ - устойчивое состояние равновесия типа фокус (узел), $O_{2}-$ состояние равновесия типа седло, $L$ - устойчивый предельный цикл, рождающийся от петли сепаратрисы седла (седло-узла) при прямом ходе параметра $\gamma$ (слева направо) и «влипающий» в эту петлю в обратном направлении. Цикл смещается вверх по цилиндру (увеличивается скорость вращения) при увеличении параметра $\gamma$. Состояния равновесия сближаются по мере приближения этого параметра к прямой $\gamma=1$ слева. 


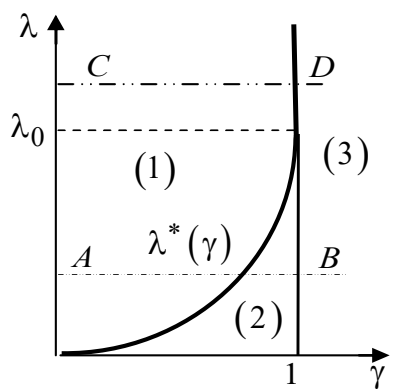

a

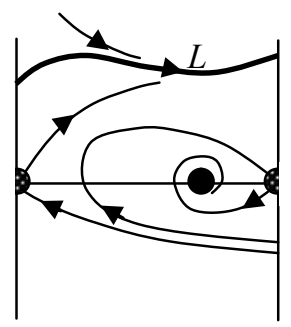

B

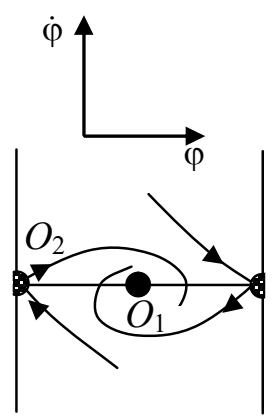

6

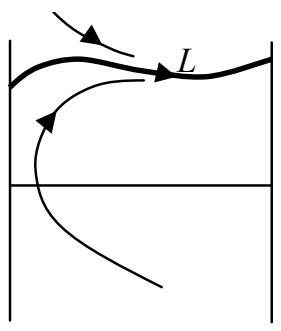

$\Gamma$

Рис. 1.1. Бифуркационная диаграмма и фазовые портреты автономного ротатора

Область параметров (1) на рис. 1.1,а соответствует глобальной устойчивости состояния равновесия $O_{1}$ на рис. 1.1,б. В этом случае ротатор (считай - маятник) из любого начального состояния с течением времени приходит в данное состояние равновесия (для маятника - нижнее). Для параметров из области (2) (рис. 1.1,a) наряду с устойчивым состоянием равновесия существует вращательный предельный цикл (рис. 1.1,в). В этом случае при одних начальных условиях ротатор приходит в состояние равновесия, при других выходит на режим стационарных вращений. Для параметров из области (3) (рис. 1.1,a) предельный цикл устойчив глобально (рис. 1.1,г). Для этих параметров ротатор из любого начального состояния с течением времени выходит на режим стационарного вращения. Как уже говорилось, при переходе из области (1) в область (2) предельный цикл рождается от петли сепаратрисы седла $\mathrm{O}_{2}$, а при переходе из области (1) в область (3) - от петли седло- 
узла. Бифуркационные значения параметров, соответствующие петле, лежат на кривой Трикоми $\lambda=\lambda^{*}(\gamma)$. Частота вращения цикла возрастает от нуля при увеличении вращающего момента $\gamma$. Это соответствует тому, что на фазовом цилиндре цикл движется вверх. Прямая $\gamma=1$ соответствует сложному состоянию равновесия, образующемуся от слияния седла $O_{2}$ и фокуса (узла) $O_{1}$. Сложное равновесие исчезает при переходе параметров через данную прямую в область (3). Фазовые портреты для бифуркационных значений параметра опущены. Для полноты картины можно добавить вторую часть бифуркационной диаграммы (см. рис. 1.1), симметричную относительно оси ординат. Для той части диаграммы будут иметь место те же самые бифуркации, но происходящие на нижней части фазового цилиндра. Это следует из инвариантности уравнения (1.1) к замене $\gamma \rightarrow-\gamma, \varphi \rightarrow-\varphi$.

Введем в рассмотрение характеристику вращения ротатора как функцию, представляющую «интегральную» информацию о его динамике при взаимодействии со своей нагрузкой, а также другими ротаторами связанной системы.

Определение. Характеристикой вращения (XB) ротатора будем называть функцию $\Omega=\lim _{T \rightarrow \infty} \frac{1}{T} \int_{0}^{T} \dot{\varphi}(\tau, \tau) d \tau$, заданную над пространством параметров системы (П1) и пространством ее начальных условий.

Как видим, ХВ представляет собой зависимость средней частоты вращения ротатора от параметров системы и ее начальных условий.

Очевидно, что любое решение системы (П1) для производной от фазы - мгновенной частоты вращения - может быть записано в виде $\dot{\varphi}\left(\tau, \tau_{0}\right)=\tilde{\dot{\varphi}}\left(\tau, \tau_{0}\right)+\dot{\varphi}^{*}\left(\tau, \tau_{0}\right)$, где $\tilde{\dot{\varphi}}-$ решение, соответствующее переходному процессу к финальному решению $\dot{\varphi}^{*}$. То есть $\quad \dot{\varphi}^{*}\left(\tau, \tau_{0}\right)=\lim _{\tau \rightarrow \infty} \dot{\varphi}\left(\tau, \tau_{0}\right), \quad \lim _{\tau \rightarrow \infty} \tilde{\dot{\varphi}}\left(\tau, \tau_{0}\right)=0 \quad$ (величина $\left|\tilde{\dot{\varphi}}\left(\tau, \tau_{0}\right)\right|$ имеет смысл уклонения решений $\dot{\varphi}\left(\tau, \tau_{0}\right)$ и $\left.\dot{\varphi}^{*}\left(\tau, \tau_{0}\right)\right)$. В силу последних соотношений получаем 


$$
\Omega=\lim _{T \rightarrow \infty} \frac{1}{T} \int_{0}^{T} \dot{\varphi}^{*}\left(\tau, \tau_{0}\right) d \tau .
$$

Решения $\dot{\varphi}^{*}\left(\tau, \tau_{0}\right)$ могут соответствовать состояниям равновесия, предельным циклам, инвариантным торам и странным аттракторам системы (П1).

Из определения видно, что исследование и построение ХВ для различных параметров системы сводится к исследованию фазового пространства связанной системы и пространства ее параметров, точнее, к классической задаче теории колебаний о разбиении пространства параметров на области, соответствующие качественно различным структурам траекторий в фазовом пространстве системы.

Для автономного ротатора решения (1.1) $\varphi^{*}\left(\tau, \tau_{0}\right)$ соответствуют состояниям равновесия и предельным циклам. На основе изложенной выше информации о динамике модели (по фазовым портретам, см. рис. 1.1) построим качественные картины ХВ автономного ротатора при изменяющемся параметре $\gamma$. Из соображений удобства и по традиции будем изображать обратную функцию $\gamma=\gamma(\Omega)$.

Допустим, что параметр $\lambda$ соответствует точке $C$, а параметр $\gamma$ изменяется вдоль $C D$ от нуля. В начале пути в системе существует глобально асимптотически устойчивое (ГАУ) состояние равновесия (см. рис. 1.1,б), и это имеет место во всем интервале $0 \leq \gamma \leq 1$. При этом $\Omega=0$, что определяет так называемую нулевую ветвь ХВ. При $\gamma=1+0$ состояние равновесия исчезает, а от петли сепаратрисы седло-узла рождается предельный цикл с предельно малой частотой $\Omega=0+0$ (см. рис. 1.1,г). Маятник начинает вращаться. Частота вращения увеличивается от нуля по мере увеличения момента $\gamma$ : предельный цикл движется вверх по фазовому цилиндру. Предельному циклу соответствует вращательная ветвь $L$ характеристики вращения. Биссектриса $\gamma=\Omega$ является асимптотой для этой кривой, что видно непосредственно из уравнения (1.1): $\dot{\varphi} \rightarrow \gamma$, если $\gamma \rightarrow \infty$. В силу инвариантности уравнения к замене $\varphi \rightarrow-\varphi, \gamma \rightarrow-\gamma$ ту же картину получаем 
в нижней полуплоскости $(\Omega, \gamma)$. В целом качественная картина ХВ имеет вид, изображенный на рис. 1.2,a.

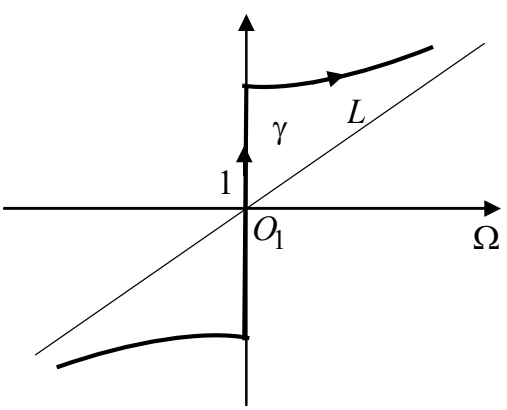

a

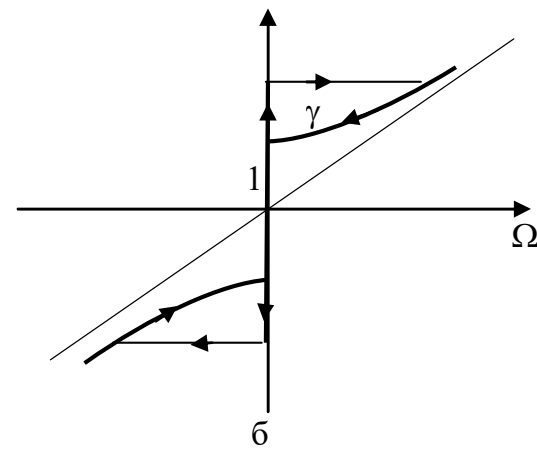

б

Рис. 1.2. ХВ при изменении $\gamma$ по $C D$ (а) и по $A B($ (б).

Допустим теперь, что параметр $\lambda$ соответствует точке $A$, а параметр $\gamma$ изменяется вдоль прямой $A B$. Как и ранее, в этом случае $\mathrm{XB}$ имеет нулевую ветвь, определяемую состоянием равновесия, которое существует в интервале $0 \leq \gamma \leq 1$. Качественное отличие в том, что при увеличении параметра в данном интервале в системе рождается устойчивый вращательный цикл (кривая Трикоми) и наряду с равновесием появляется возможность вращений. При этом к моменту исчезновения состояния равновесия предельный цикл, двигаясь вверх по фазовому цилиндру, приобретает вполне определенную частоту вращения $\Omega>0$. Это приводит к тому, что при $\gamma=1+0$ происходит скачок частоты вращения с нулевой ветви на вращательную ветвь ХВ. При дальнейшем увеличении $\gamma$ поведение вращательной ветви ХВ аналогично описанному выше случаю. Другое дело - при уменьшении параметра $\gamma$, когда ротатор находится на предельном цикле и вплоть до исчезновения последнего «не замечает» рождения состояния равновесия. Частота вращения цикла уменьшается и становится нулевой при $\gamma=\lambda^{*}$, в момент «влипания» цикла в петлю сепаратрисы седла. При этом вращательная ветвь примыкает к нулевой ветви ХВ. На картине имеется выраженная гистерезисная петля. Полная картина гистерезисной ХВ с учетом симметричной части бифуркаци- 
онной диаграммы изображена на рис. 1.2,б. По бифуркационной диаграмме видно, что данными двумя типами исчерпываются все возможные типы качественных картин ХВ автономного ротатора.

Для подробного исследования вращательных движений ротаторов далее будут использоваться методы малого параметра [13-16], а также метод точечных отображений [17], тесно связанный с методом усреднения. Метод усреднения будет применяться на основе специальных преобразований систем из класса (П1), изложенных в Приложении I.

Рассмотрим подробно вращательные движения ротатора, начиная с демонстрации алгоритма преобразований уравнения ротатора к так называемой стандартной форме.

Задача: преобразовать уравнение (1.1) к эквивалентной системе вида

$$
\begin{aligned}
& \dot{\xi}=\mu \Xi(\xi, \varphi), \\
& \dot{\varphi}=\omega+\mu \Phi(\xi, \varphi),
\end{aligned}
$$

где $\xi$ - некоторая новая переменная, $\mu=I^{-1}, \omega$ - параметр, подлежащий определению, $\Xi(\xi, \varphi)$ и $\Phi(\xi, \varphi)$ - ограниченные по $\varphi$ (периодические) функции, которые также подлежат определению.

Решение: подставляем второе уравнение системы (1.2) в уравнение (1.1), получаем

$$
\frac{\partial \Phi}{\partial \varphi}(\omega+\mu \Phi)+\frac{\partial \Phi}{\partial \xi} \dot{\xi}+\left(1+F_{1}(\varphi)\right)(\omega+\mu \Phi)+F_{2}(\varphi)=\gamma
$$

Из этого уравнения выбираем параметр $\omega$ и неизвестные функции, учитывая естественное условие ограниченности искомых функций по фазовой переменной ф и требуемый вид системы (1.2). Из условия ограниченности получаем $\omega=\gamma$. С учетом вида второго уравнения системы (1.2) получаем уравнение, определяющее функцию $\Phi(\xi, \varphi)$ (отделяем члены уравнения, не содержащие сомножитель $\mu)$ :

$$
\frac{\partial \Phi}{\partial \varphi} \omega+\omega F_{1}(\varphi)+F_{2}(\varphi)=0
$$


Для уравнения (1.4) наиболее простое решение имеет вид:

$$
\Phi(\varphi, \xi)=-\int F_{1}(\varphi) d \varphi-\frac{1}{\omega} \int F_{2}(\varphi) d \varphi+\xi .
$$

При этом оставшиеся члены (1.3) определяют уравнение для функции $\Xi(\xi, \varphi)$ :

$$
\Xi(\xi, \varphi)=-\Phi \frac{\partial \Phi}{\partial \varphi}-\left(1+F_{1}(\varphi)\right) \Phi
$$

В случае функций $F_{1}(\varphi)=\varepsilon \cos \varphi, F_{2}(\varphi)=\sin \varphi$ получаем

$$
\begin{aligned}
& \dot{\xi}=\mu\left(-\varepsilon \sin \varphi+\frac{1}{\omega} \cos \varphi+\xi\right)\left(1-\frac{1}{\omega} \sin \varphi\right), \\
& \dot{\varphi}=\omega+\mu\left(-\varepsilon \sin \varphi+\frac{1}{\omega} \cos \varphi+\xi\right) .
\end{aligned}
$$

Заметим, что система (1.5) эквивалентна (!) уравнению (1.1). С другой стороны, если $\mu$ - малый параметр (как правило, уровень малости параметра в асимптотических методах не обсуждается, хотя и существуют работы по его оценке), то система (1.5) имеет «стандартную форму системы с быстровращающейся фазой» $(\varphi)$. Техника усреднения таких систем разработана во всех подробностях [18], и мы на ней останавливаться не будем. Усредняя (1.5) до членов порядка $\mu^{3}$, получаем усредненную систему вида

$$
\begin{aligned}
& \dot{\xi}=-\mu\left(\frac{\varepsilon}{2 \omega}+\xi\right)+\mu^{2}\left(\frac{\varepsilon^{2}}{2 \omega}+\frac{\varepsilon}{2 \omega^{2}} \xi\right), \\
& \dot{\varphi}=\omega+\mu \xi-\mu^{2}\left(\frac{\varepsilon^{2}}{2 \omega}+\frac{1}{2 \omega^{3}}\right) .
\end{aligned}
$$

В уравнениях (1.6) за усредненными переменными сохранены прежние обозначения.

Известно, что состоянию равновесия системы (1.6) соответствует предельный цикл системы (1.2) и, соответственно, уравнения (1.1) вместе с условиями устойчивости. Как видим, состояние равновесия единственно и устойчиво, его координата 
$\xi_{0}=\frac{-\frac{\varepsilon}{2 \omega}(1-\mu \varepsilon)}{1-\frac{\mu \varepsilon}{2 \omega^{2}}}$. Подставляя это выражение во второе уравнение (1.6), получаем выражение для вращательной ветви ХВ в явном виде (точность $\mu^{3}$ ):

$$
\Omega=\gamma-\frac{\mu \varepsilon}{2 \gamma}-\frac{\mu^{2}}{4 \gamma^{3}}\left(\varepsilon^{2}+2\right) .
$$

На рис. 1.3 представлен аналитический результат (1.7) в сравнении с результатом численного эксперимента [5]. Видно, что даже при немалых значениях параметра $\mu(\mu=1 / 2)$ имеется хорошее качественное и количественное сходство (известное «чудо малого параметра»).

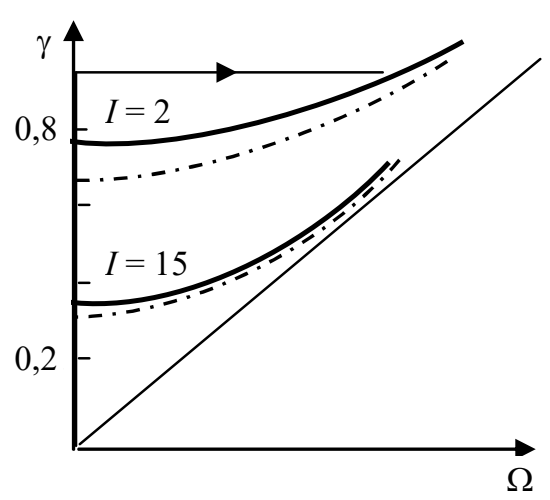

Рис. 1.3. Характеристика вращения: сплошная линия - численный эксперимент, пунктирная - аналитический результат

Замечание. Далее нас будут интересовать качественные особенности динамики систем, поэтому мы не будем проводить операции усреднений до порядка выше первого. Правые части усредненных систем первого приближения находятся просто как средние соответствующих правых частей на периоде быстрой фазы. Такие укороченные системы содержат все интересующие нас качественные особенности исходных систем. Обоснованием этому является 
введенное А. А. Андроновым $[1,19]$ понятие грубости (структурной устойчивости) динамических систем.

Обратимся к примерам физических систем, динамика которых моделируется уравнением ротатора.

Пример 1: маятник Фруда [20], рис. 1.4. Муфта маятника насажена на вал, вращающийся с постоянной угловой скоростью $\Omega$. Между муфтой и валом имеется вязкое трение. Момент силы трения, действующий на маятник, $\delta(\Omega-\dot{\varphi})$, момент силы тяжести равен $m g l \sin \varphi, m l^{2}$ - момент инерции маятника. Все вместе дает уравнение движения маятника:

$$
m l^{2} \ddot{\varphi}+\delta \dot{\varphi}+m g l \sin \varphi=\delta \Omega .
$$

В результате преобразования времени и переобозначения параметров это уравнение сводится к уравнению (1.1). В данном случае характеристика вращения имеет буквальный смысл зависимости угловой скорости вращения маятника от внешнего движущего момента, пропорционального скорости вращения вала. По существу, обо всех особенностях динамики этой системы уже сказано выше.

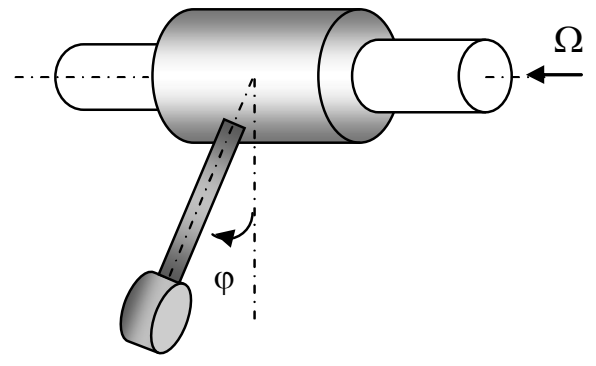

Рис. 1.4. Маятник Фруда

Пример 2: синхронная электрическая машина. Как правило, эта физическая система описывается шестимерной моделью [6,21]. Однако в силу различия в порядках величин скоростей изменения электрических и механических процессов движение ее ротора может описываться динамической системой вида [12]

$$
I \ddot{\varphi}+(\alpha+\beta \cos 2 \varphi-\delta \sin \varphi) \dot{\varphi}+\sin \varphi=T .
$$


Здесь $I$ - момент инерции ротора; $\alpha, \beta, \delta$ - коэффициент вязкого трения и коэффициенты электрического демпфирования соответственно; $T$ - постоянный движущий момент со стороны нагрузки и магнитного поля; $\varphi$ - угол между направлениями магнитных полей статора и ротора. Как видно, это уравнение представляет ротатор (1.1).

Пример 3: система фазовой синхронизащии [10, 11], рис. 1.5.

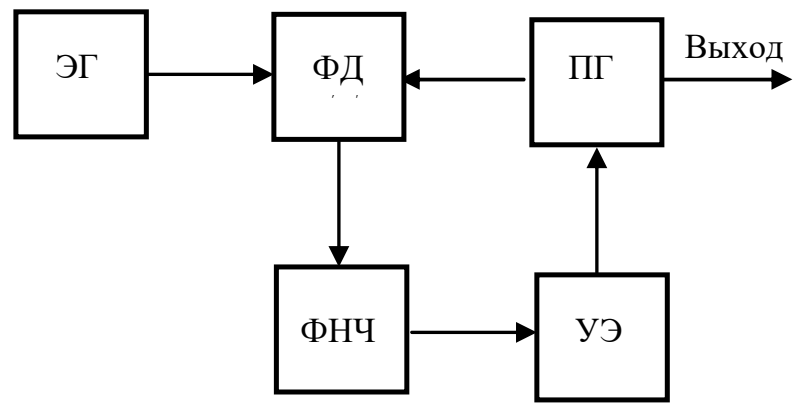

Рис. 1.5. Блок-схема системы фазовой синхронизации: ЭГ - эталонный генератор, ФД - фазовый детектор, ПГ - подстраиваемый генератор, ФНЧ - фильтр нижних частот, УЭ - управляющий элемент

При синусоидальной характеристике фазового детектора и коэффициенте передачи фильтра нижних частот $K(p)$, где $p$ - оператор дифференцирования, общее уравнение этой системы имеет вид:

$$
p \varphi+\Omega_{\mathrm{y}} K(p) \sin \varphi=\Omega_{\mathrm{H}} .
$$

Здесь $\Omega_{\mathrm{y}}, \Omega_{\mathrm{H}}$ - полоса удержания и начальная расстройка частот подстраиваемого и эталонного генераторов, а $\varphi-$ их разность фаз. В случае фильтра первого порядка вида $K(p)=\left(T_{1} p+1\right) /(T p+1)$, где $T_{1}, T$ - постоянные фильтра, это уравнение элементарными преобразованиями сводится к уравнению (1.1).

Пример 4: сверхпроводящий джозефсоновский переход (контакт) [3-5]. В 1962 г. Б. Джозефсон теоретически предсказал существование двух эффектов в динамическом поведении соединения двух сверхпроводников [3] (рис. 1.6). Первый заключается 
в том, что через такое соединение возможно протекание сверхтока $I_{s}$, не вызывающего падения напряжения на переходе.

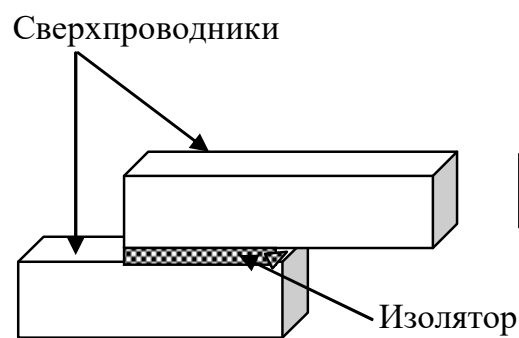

a
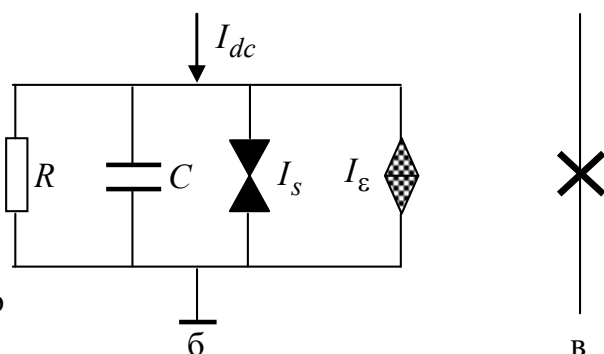

B

Рис. 1.6. Сверхпроводящий переход (a); электрическая схема резистивной модели (б); схемное обозначение (в).

Второй эффект состоит в том, что если напряжение на переходе вызывается какими-либо причинами, то его значение $V$ связано с частотой электромагнитного поля в пространстве перехода фундаментальными константами: $\omega=2 \mathrm{eV} / \mathrm{h}$ (соотношение Джозефсона), где $e-$ заряд электрона, $h$ - постоянная Планка. В простейшем случае $I_{s}=I_{c} \sin \varphi$, где $\varphi-$ разность фаз квантово-механических волновых функций параметра порядка сверхпроводников [3]. Динамическая (резистивная) модель перехода введена в [22]. Согласно этой модели эквивалентная радиотехническая схема перехода с заданным внешним током $I$ представляет параллельное соединение емкости, сопротивления и «генератора» сверхтока (см. рис. 1.6,б). Джозефсоном указана еще одна компонента тока $I_{\varepsilon}=\varepsilon V \cos \varphi$, текущего через контакт. В данной модели выражение для полного тока контакта с учетом соотношения Джозефсона в размерных параметрах и времени имеет вид:

$$
\frac{h}{2 e} C \ddot{\varphi}+\frac{h}{2 e R}(1+\varepsilon \cos \varphi) \dot{\varphi}+I_{c} \sin \varphi=I_{d c},
$$

где $I_{d c}-$ постоянный ток внешнего источника, считающийся заданным. В результате введения безразмерного времени $\Omega_{d} t=\tau$, 
где $\Omega_{d}=\frac{2 \pi I_{c} R}{\Phi_{0}}-$ джозефсоновская частота, $\Phi_{0}=h / 2 e-$ квант магнитного потока, это уравнение сводится к уравнению (1.1). В безразмерном времени производная $\dot{\varphi}$ имеет смысл напряжения с постоянной составляющей $\langle\dot{\varphi}\rangle_{t}=\omega$, а параметр $\gamma$ - смысл постоянного тока, текущего через контакт. Таким образом, введенная выше характеристика вращения ротатора в случае сверхпроводящего перехода имеет смысл его вольтамперной характеристики (BAX). Соответственно, нулевая ветвь ХВ - сверхпроводящая ветвь ВАХ, вращательная ветвь - резистивная ветвь ВАХ. В данном случае прослеживается полная механическая и квантовомеханическая аналогия.

\section{2. Синхронизация и хаотические вращения неавтономного ротатора}

Неавтономным ротатором называем динамическую систему дифференциальные уравнения вида

$$
\begin{aligned}
& I \ddot{\varphi}+\left(1+F_{1}(\varphi)\right) \dot{\varphi}+F_{2}(\varphi)=\gamma+f(\psi), \\
& \dot{\psi}=\omega_{0},
\end{aligned}
$$

где $f(\psi)$ - внешнее воздействие с нулевым средним $\langle f(\psi)\rangle_{\psi}=0$. Явное время «спрятано» добавлением второго уравнения. Примерами физических систем, моделируемых уравнениями (1.8), являются: маятник Фруда, вал которого испытывает вращательноколебательное движение; сверхпроводящий переход, находящийся в СВЧ-поле; система фазовой синхронизации с аддитивной помехой и многие другие системы. По числу приложений динамическую систему (1.8) можно отнести к числу базовых моделей нелинейной динамики (теории нелинейных колебаний).

Визуальная простота модели обманчива. Ее аналитическое исследование затруднено сложностью фазового пространства (в общем случае - значений параметров), связанной с существованием гомоклинических структур, порождающих хаотические (странные) аттракторы. По этой причине традиционные аналитические методы 
в данном случае во многом теряют свою эффективность. Аналитические результаты исследования уравнений (1.8) немногочисленны; основная их часть получена качественно-численными методами и касается главным образом динамики систем фазовой синхронизации на границе области захвата синхронизации. Ниже сделаем попытку внести посильный вклад в копилку аналитических результатов, касающихся вращательных движений модели. В числе прочего в рамках метода усреднения укажем конкретные области параметров, для которых вращения ротатора приобретают хаотический характер. Исследование проводим в квазилинейном случае $I^{-1}=\mu<<1$.

Как правило, при гармоническом воздействии на квазилинейную систему «сильными» являются только целые, гармонические резонансы на частотах $n \omega_{0}, n$ - целое. В смысле усреднительной процедуры резонанс называем «сильным», если он «виден» в усредненной системе первого приближения. Субгармонические резонансы в этом смысле являются «слабыми». Известно также, что среди гармонических резонансов наиболее значимым является главный (первый) резонанс $n=1$, называемый простой синхронизацией. В других случаях синхронизация называется кратной.

1. Исследуем простую принудительную синхронизацию вращений ротатора. Рассматриваем случай «умеренных» амплитуд внешнего воздействия и решаем задачу преобразования системы (1.8) к эквивалентной системе в стандартной форме:

$$
\begin{aligned}
& \dot{\xi}=\mu \Xi(\xi, \varphi, \psi), \\
& \dot{\varphi}=\omega_{0}+\mu \Phi(\xi, \varphi, \psi), \\
& \dot{\psi}=\omega_{0} .
\end{aligned}
$$

В отличие от автономного случая, когда «порождающая» частота ротатора (частота ротатора при $\mu=0$ ) находилась в процессе решения задачи, здесь она определена рассматриваемым резонансом. Остается задача о функциях $\Phi(\xi, \varphi, \psi)$ и $\Xi(\xi, \varphi, \psi)$.

Подставляем второе уравнение системы (1.9) в уравнение (1.8) и получаем 


$$
\begin{gathered}
\frac{\partial \Phi}{\partial \varphi}\left(\omega_{0}+\mu \Phi\right)+\frac{\partial \Phi}{\partial \psi} \omega_{0}+\frac{\partial \Phi}{\partial \xi} \dot{\xi}+ \\
+\left(1+F_{1}(\varphi)\right)\left(\omega_{0}+\mu \Phi\right)+F_{2}(\varphi)=\gamma+f(\psi) .
\end{gathered}
$$

Полагаем, что $\gamma-\omega_{0}=\mu \Delta$. Это условие соответствует зоне главного резонанса по параметру $\gamma$ (близость частот). Отделяя непропорциональные $\mu$ члены уравнения (1.10), получаем уравнение, определяющее функцию $\Phi(\xi, \varphi, \psi)$ :

$$
\frac{\partial \Phi}{\partial \varphi} \omega_{0}+\frac{\partial \Phi}{\partial \psi} \omega_{0}=-\omega_{0} F_{1}(\varphi)-F_{2}(\varphi)+f(\psi) .
$$

Это уравнение имеет решение:

$$
\Phi(\varphi, \xi)=-\int F_{1}(\varphi) d \varphi-\frac{1}{\omega_{0}} \int F_{2}(\varphi) d \varphi+\frac{1}{\omega_{0}} \int f(\psi) d \psi+\xi .
$$

Оставшиеся члены в уравнении (1.10) определяют функцию $\Xi(\xi, \varphi, \psi)$ :

$$
\Xi(\xi, \varphi, \psi)=\Delta-\Phi\left(1+F_{1}(\varphi)+\frac{\partial \Phi}{\partial \varphi}\right) .
$$

Искомые функции найдены. После введения фазовой расстройки $\eta=\varphi-\psi$ система (1.8) приобретает искомый стандартный вид системы с одной быстровращающейся фазой:

$$
\begin{aligned}
& \dot{\xi}=\mu \Xi(\xi, \varphi, \eta), \\
& \dot{\eta}=\mu \Phi(\xi, \varphi, \eta), \\
& \dot{\varphi}=\omega_{0}+\mu \Phi(\xi, \varphi, \eta) .
\end{aligned}
$$

Исследуем динамику системы (1.12) с конкретным видом функций: $F_{1}(\varphi)=\varepsilon \cos \varphi, F_{2}(\varphi)=\sin \varphi, f(\psi)=A \sin \psi$. В этом случае

$$
\Phi(\varphi, \xi)=-\varepsilon \sin \varphi+\frac{1}{\omega_{0}} \cos \varphi-\frac{A}{\omega_{0}} \cos \psi+\xi, \psi=\varphi-\eta,
$$




$$
\Xi(\xi, \varphi, \psi)=\Delta-\left(-\varepsilon \sin \varphi+\frac{1}{\omega_{0}} \cos \varphi-\frac{A}{\omega_{0}} \cos \psi+\xi\right)\left(1-\frac{1}{\omega_{0}} \sin \varphi\right) .
$$

Проводя усреднение системы (1.12), получаем укороченную систему вида

$$
\begin{aligned}
& \dot{\xi}=\mu\left(-\xi+\Delta-\frac{\varepsilon}{2 \omega_{0}}-\frac{A}{2 \omega_{0}^{2}} \sin \eta\right), \\
& \dot{\eta}=\mu \xi \\
& \dot{\varphi}=\omega_{0}+\mu \xi .
\end{aligned}
$$

Первые два уравнения (1.13) не зависят от третьего и относительно переменной $\eta$ при замене времени $\mu \sqrt{\frac{A}{2 \omega_{0}^{2}}} \tau=\tau_{n}$ сводятся к виду уже известного нам уравнения автономного ротатора (!):

$$
\ddot{\eta}+\lambda^{r} \dot{\eta}+\sin \eta=\gamma^{r}
$$

где $\lambda^{r}=\sqrt{\frac{2 \omega_{0}^{2}}{A}}, \gamma^{r}=\left(\Delta-\frac{\varepsilon}{2 \omega_{0}}\right) \frac{2 \omega_{0}^{2}}{A}$.

Заметим, что подобная ситуация будет повторяться и далее в этом особенность проводимых преобразований систем. А именно: если имеется некоторая автономная система, то в неавтономном случае усредненная система будет сводиться к виду этой автономной системы. Остается лишь адекватно интерпретировать свойства усредненной системы на исходную систему.

Таким образом, имеется возможность использования ранее полученных результатов при условии ответа на два вопроса. Первый: какова связь траекторий (1.14) с траекториями системы (1.8)? Второй: все ли бифуркации уравнения (1.14) можно однозначно интерпретировать на соответствующие бифуркации системы (1.8)? Для ответа на эти вопросы следует обратиться к понятию грубости динамических систем, к сведениям из теории бифуркаций о гомоклинической траектории седла и бифуркациям двумерного тора $[23,24]$, а также к смыслу процедуры усреднения как замены переменных [14, 18$]$.

Не вдаваясь в подробности, можно утверждать, что если Г траектория усредненной системы с ненулевыми характеристиче- 
скими показателями, достаточно отделенными от мнимой оси, то она соответствует траектории $\Gamma \times S^{1}$ исходной системы вместе с характером устойчивости. То есть грубому состоянию равновесия усредненной системы будет соответствовать предельный цикл исходной, а предельному циклу будет соответствовать двумерный тор [17] с квазипериодической обмоткой (квазипериодические движения системы) или с субгармоническим резонансным циклом. Однако имеется топологическая эквивалентность фазового портрета усредненной системы и портрета дискретных траекторий в пространстве отображения Пуанкаре секущей плоскости $\varphi(\bmod 2 \pi)=$ const в себя через период быстрой фазы, построенного для исходной системы. Таким образом, области параметров (1), (2), (3) на рис. 1.1 практически целиком можно переносить на искомую бифуркационную диаграмму в плоскости параметров $\left(\gamma^{r}, \lambda^{r}\right)$ : $\lambda \rightarrow \lambda^{r}, \gamma \rightarrow \gamma^{r}$ (рис. 1.7,a).

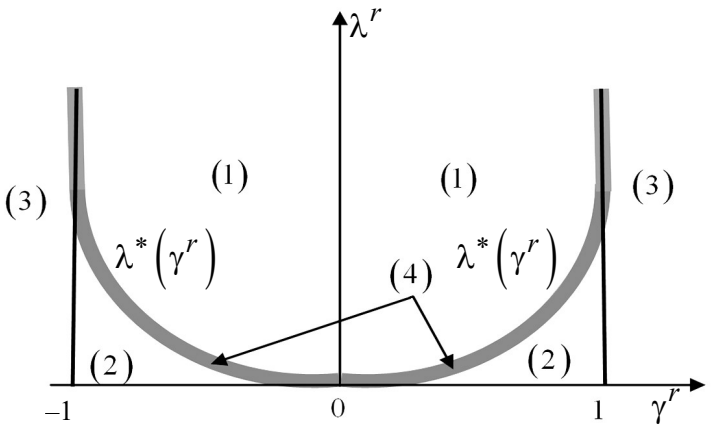

a

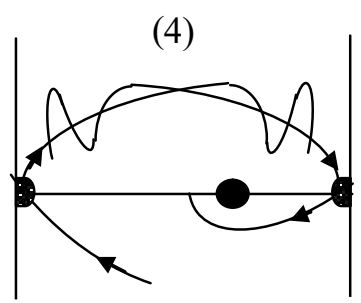

б

Рис. 1.7. Бифуркационная диаграмма для параметров в зоне главного резонанса (a); гомоклиническая структура для области (4) (б).

То же самое относится и к изображенным на рис. 1.1 фазовым портретам, на которых следует сделать переобозначения $\varphi \rightarrow \eta$, $\dot{\varphi} \rightarrow \xi$, а траектории считать траекториями точечного отображения $(\mathrm{TO}):(\xi, \eta)_{\varphi} \rightarrow(\bar{\xi}, \bar{\eta})_{\varphi+2 \pi}$ для системы (1.12), эквивалентной уравнению (1.8). В таком случае состояния равновесия прошлой систе- 
мы становятся неподвижными точками ТО - предельными циклами системы (1.12), а предельные циклы - двумерными торами.

Таким образом, в области параметров (1) на рис. 1.7,а существуют две неподвижные точки: одна из них устойчивая - устойчивый предельный цикл, соответствующий синхронизации ротатора внешней силой, а вторая является седлом. Эта область является областью захвата синхронизации, когда синхронизм вращений наступает при любом начальном условии. В области параметров (2), кроме названных неподвижных точек, существует инвариантный тор. Движения системы на этом торе могут быть как квазипериодическими (биения), так и периодическими (при «шевелении» параметра) с периодом вращения, находящемся в дробном соотношении с периодом внешней силы (субгармонический резонанс). В данном случае метод усреднения подобные мелкие детали не учитывает. Однако поскольку бифуркации систем на двумерном торе полностью изучены, то сказанное имеет силу утверждения. Интервал существования областей (1) и (2), выражаемый неравенством $\left|\gamma^{r}\right| \leq 1$, т. е. интервал существования устойчивой неподвижной точки ТО, является интервалом (полосой) удержания синхронизации. В области параметров (3) единственным притягивающим множеством является двумерный тор. В зависимости от параметров системы на этом торе имеется или квазипериодическая обмотка - режим биений ротатора, или предельный цикл, соответствующий субгармонической синхронизации. Надо сказать, что такие резонансы в общем случае «мало живучи» при изменении параметров системы. В общем случае картина поведения системы при изменении параметра в области (3) состоит из попеременной смены режимов биений субгармоническими резонансами с различным отношением периодов к периоду внешнего воздействия.

Как видим, на рис. 1.7,а вместо кривой Трикоми - петли сепаратрисы седла - на ее месте располагается область (4), изображенная в виде узкой полосы (толстая кривая Трикоми). Поясним. Как известно, петля сепаратрисы седла в фазовом пространстве образуется от слияния («слипания») входящего и выходящего многообразий - сепаратрис седла. Это является единственной возможностью поведения для фазовых траекторий. В отличие от фазовых траекторий пересечение траекторий точечного отображения не запрещено. При этом пересечение входящего и выходящего многообразий седла 
(рис. $1.7,6)$ является случаем общего положения. С другой стороны, пересечение, возникшее однажды, влечет за собой бесконечное число точек пересечения. Это так, потому что точка пересечения принадлежит и тому, и другому многообразиям одновременно, а, следовательно, она и в последующих итерациях ТО должна принадлежать обоим многообразиям. То есть каждая точка пересечения является прообразом другой точки, и последняя тоже является точкой пересечения инвариантных многообразий. Входящее и выходящее многообразия осциллируют, пересекая друг друга в бесконечном числе точек с шагом осцилляций, стремящимся к нулю по мере приближения к седловой точке с точки пересечения. Точки пересечения многообразий называются гомоклиническими точками. Множество гомоклинических точек составляет две последовательности: одна последовательность стремится к седлу при $t \rightarrow+\infty$, а вторая стремится к нему же при $t \rightarrow-\infty$. Совокупность гомоклинических точек является следом фазовой траектории, двоякоасимптотической к седлу, - гомоклинической кривой Пуанкаре. Доказано, что в окрестности гомоклинических кривых содержится счетное множество седловых предельных циклов [24], которые имеют свои гомоклинические кривые, и т.д. Гомоклиническая структура как совокупность гомоклинических кривых и бесконечного множества седловых предельных циклов в рассматриваемом случае является притягивающей для соседних траекторий и, тем самым, представляет собой хаотический аттрактор. Надо сказать, что в подобных структурах могут быть и устойчивые циклы, но они имеют настолько малые области притяжения, что естественные шумы, которые всегда присутствуют в реальной системе, препятствуют их реализации. Другими словами, в реальных условиях гомоклинические структуры являются «настоящими» странными аттракторами. Вторым механизмом возникновения динамического хаоса в рассматриваемой системе является бифуркация разрушения двумерного тора $[23,24]$, существующего в области (3) при приближении параметров к области (4). Если в автономном случае для параметров на кривой Трикоми цикл «влипал» в петлю сепаратрисы седла, то для тора это невозможно: тор сначала теряет гладкость, а затем разрушается с дальнейшим образованием на его месте хаотического аттрактора. При изменении параметров в области (4) описанные аттракторы объединяются. 
Таким образом, область параметров (4) соответствует хаотической динамике ротатора. Область достаточно узкая, ее ширина $\sim \mu$, и она стягивается к кривой Трикоми при уменьшении малого параметра. Заметим, что существование петель сепаратрис седел в усредненной системе является практически гарантией существования гомоклинических структур в исходной системе и может играть роль критерия.

Построим фрагмент характеристики вращения неавтономного ротатора в зоне главного резонанса, заметив, что из определения ХВ и принципа усреднения следует $\left\langle\dot{\varphi}^{*}\right\rangle_{t}=\omega_{0}+\left\langle\dot{\eta}^{*}\right\rangle_{t}$, а из обозначения параметров $\gamma^{r} \sim \Delta \sim \gamma$. Рассматриваем те значения $\lambda^{r}=$ const, для которых соответствующая прямая на рис. 1.7,a пересекает области (1), (4), (2), (3) при увеличении параметра $\gamma^{r}$ от нуля. Полную картину ХВ получаем, пользуясь центральной симметрией этой кривой (первое приближение) в рассматриваемой зоне.

Допустим, что параметр $\gamma^{r}$ находится в области (1). В режиме синхронизации вращений ротатора $\left\langle\dot{\eta}^{*}\right\rangle_{t}=0$, а значит, $\left\langle\dot{\varphi}^{*}\right\rangle_{t}=\omega_{0}$. В режиме синхронизации ротатор «держится» до тех пор, пока не исчезнет устойчивая неподвижная точка на линии $\gamma^{r}=1$. Таким образом, на $\mathrm{XB}$, на частоте внешнего воздействия $\Omega=\omega_{0}$, имеется вертикальный участок - резонансная ступень, величина которой определяется неравенством $\left|\gamma^{r}\right| \leq 1$, или в раскрытом виде $\omega_{0}-\mu \frac{A}{2 \omega_{0}^{2}} \leq \gamma \leq \omega_{0}+\mu \frac{A}{2 \omega_{0}^{2}}$. Середина ступени находится на вращательной ветке ХВ автономного ротатора (рис. 1.8).

При значении $\left|\gamma^{r}\right|=1+0$ (при переходе параметров из области (2) в область (3)) предельный цикл исчезает и ротатор скачком переходит из режима синхронизации в режим квазипериодических биений, соответствующий тору $T^{2}$. В этом случае $\left\langle\dot{\eta}^{*}\right\rangle_{t} \neq 0$, при- 
чем $\left\langle\dot{\eta}^{*}\right\rangle_{t}$ возрастает с увеличением $\gamma$ и убывает с уменьшением этого параметра. В обоих случаях на торе может возникать последовательность субгармонических резонансов, отражающихся на ХВ в виде ступенек небольшой величины (на рис. 1.8 они не показаны). При вхождении параметра $\gamma$ в область (4) тор теряет гладкость, разрушается, и на его месте образуется странный аттрактор [23]. Далее к этому аттрактору подмешивается гомоклиническая структура седловой неподвижной точки главного резонанса. Происходит всплеск интенсивности хаотических вращений. При дальнейшем уменьшении параметра странный аттрактор теряет свойство притяжения и ротатор скачком возвращается на резонансную ступень. Слева от резонансной ступени динамика ротатора претерпевает те же самые бифуркации и поведение ХВ аналогично. Исключая участки, определяемые странными аттракторами, ХВ в зоне синхронизации похожа на ХВ автономного ротатора. При движении системы на хаотическом аттракторе структура ХВ является сложной. Подробности ее поведения в таких случаях обсудим в конце раздела.

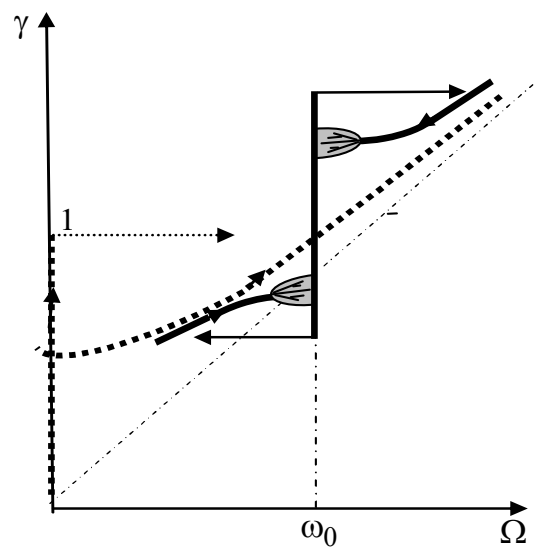

Рис. 1.8. ХВ неавтономного ( - ) ротатора в зоне главного резонанса.

2. Рассматриваем динамику ротатора при больших амплитудах внешнего воздействия $A \sim \mu^{-1}>>1$. В данном случае нас будет интересовать динамика системы в зоне произвольного гармонического резонанса. Этот случай технически сложнее предыдущего. Для 
сведения уравнения (1.8) к стандартной форме требуется предварительное преобразование.

Для простоты выкладок положим $\varepsilon=0$ и выполним замену переменных: $\varphi=b \sin \left(\psi+\psi_{0}\right)+\theta, \quad \psi+\psi_{0}=\psi_{n} \quad$ (далее индекс $n$ опускаем), $\quad \psi_{0}=\operatorname{arctg} \frac{1}{\omega_{0} I}, \quad b=\frac{A}{\omega_{0} \sqrt{\omega_{0}^{2} I^{2}+1}} \approx \frac{A}{\omega_{0}^{2} I}$. В результате

сводится к эквивалентному уравнению вида

$$
I \ddot{\theta}+\dot{\theta}+\sin (\theta+b \sin \psi)=\gamma .
$$

Известно, что

$$
\begin{aligned}
\sin (\theta+ & b \sin \psi)=J_{0} \sin \theta+\sum_{m=1}^{\infty} J_{2 m}\{\sin (2 m \psi+\theta)-\sin (2 m \psi-\theta)\}+ \\
& +\sum_{k=0}^{\infty} J_{2 k+1}\{\sin ((2 k+1) \psi+\theta)+\sin ((2 k+1) \psi-\theta)\},
\end{aligned}
$$

где $J_{l}=J_{l}(b)$ - функции Бесселя первого рода с целочисленным аргументом. Преобразуя теперь уравнение (1.15) по уже известному правилу, получаем эквивалентную систему в стандартной форме

$$
\begin{aligned}
& \dot{\xi}=\mu^{1 / 2}\left(\gamma-n \omega_{0} \pm J_{n}(b) \sin \eta\right)-\mu \xi-\mu \xi \frac{\partial \Phi}{\partial \theta}-\mu^{3 / 2}\left(1+\frac{\partial \Phi}{\partial \theta}\right), \\
& \dot{\theta}=n \omega_{0}+\mu^{1 / 2} \xi+\mu \Phi(\theta, \psi), \\
& \dot{\psi}=\omega_{0},
\end{aligned}
$$

где знак «+» для четных чисел $n$, а «-» для нечетных. При этом уравнение, определяющее функцию $\Phi$, имеет вид

$$
\begin{gathered}
n \omega_{0} \frac{\partial \Phi}{\partial \theta}+\omega_{0} \frac{\partial \Phi}{\partial \psi}=-J_{0} \sin \theta- \\
-\sum_{m=1}^{\infty} J_{2 m}\left\{\sin (2 m \psi+\theta)-\delta_{n, 2 m} \sin (2 m \psi-\theta)\right\}- \\
-\sum_{k=0}^{\infty} J_{2 k+1}\left\{\sin ((2 k+1) \psi+\theta)+\delta_{n, 2 k+1} \sin ((2 k+1) \psi-\theta)\right\},
\end{gathered}
$$

где $\delta_{n, l}=1$, если $n \neq l$, и $\delta_{n, l}=0$, если $n=l$. 
Решение (1.17) не представляет особого труда. После введения фазовой расстройки $\eta=\theta-n \psi$ и усреднения системы (1.16) до членов $\sim \mu$ получаем следующую усредненную систему:

$$
\begin{aligned}
& \dot{\xi}=\mu^{1 / 2}\left(\gamma-n \omega_{0} \pm J_{n}(b) \sin \eta\right)-\mu \xi, \\
& \dot{\eta}=\mu^{1 / 2} \xi, \\
& \dot{\theta}=n \omega_{0}+\mu^{1 / 2} \xi .
\end{aligned}
$$

Первые два уравнения этой системы при замене времени $\sqrt{\mu\left|J_{n}(b)\right|} \tau=\tau_{n}$ сводятся к знакомому нам уравнению

$$
\ddot{\eta}+\lambda_{n}^{r} \dot{\eta} \pm \sin \eta=\gamma_{n}^{r},
$$

где $\lambda_{n}^{r}=\sqrt{\frac{\mu}{\left|J_{n}(b)\right|}}, \gamma_{n}^{r}=\frac{\gamma-n \omega_{0}}{\left|J_{n}(b)\right|}$.

Заметим, что знаки перед функцией не имеют принципиального значения. При необходимости от знака «-» освобождаемся преобразованием $\eta \rightarrow \eta+\pi$. В (1.18) содержится интересный факт: если в этом уравнении «забыть», что $A$ является «большим» параметром, то аргумент функции Бесселя достаточно мал и $J_{1}\left(\frac{A}{\omega_{0}^{2} I}\right) \approx \frac{A}{2 \omega_{0}^{2} I}$. При этом уравнение (1.18) переходит в уравнение (1.14). Это можно отнести к очередному «чуду малого параметра», когда результат, полученный при одних условиях, остается справедливым и при других.

Проведем анализ уравнения (1.18). В общем случае при больших амплитудах внешнего воздействия существенными являются все гармонические резонансы квазилинейного ротатора. Величина резонансных ступеней равна $2\left|J_{n}(b)\right|$. Без учета странных аттракторов (области (4)) качественная картина XВ в окрестности каждого из этих резонансов подобна ХВ автономного ротатора - с гистерезисной петлей или без нее. Соседние резонансные ступени на ХВ могут перекрываться. Величина перекрытия соседних резонансов $\delta \gamma$ определяется неравенством $(n+1) \omega_{0}-\left|J_{n+1}(b)\right|<\delta \gamma<n \omega_{0}+\left|J_{n}(b)\right|$. 
В этом интервале тот или другой резонансы реализуются в зависимости от начальных условий системы. Из неравенства и свойств функций Бесселя [25] следует, что перекрытие резонансов отсутствует при $\omega_{0}>1,2$, а если $\omega_{0}$ достаточно мало, то перекрытие существует не только для соседних резонансов. На рис. 1.9 приведены графики некоторых функций Бесселя.

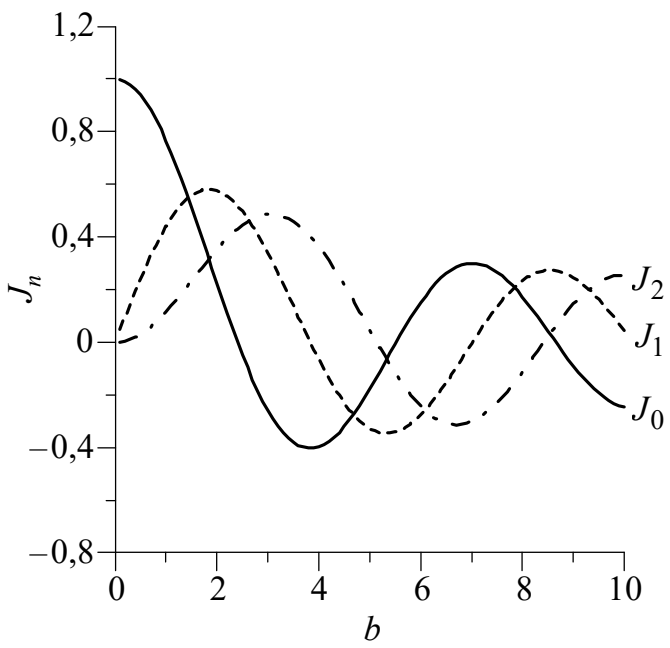

Рис. 1.9. Графики функций Бесселя.

Из свойств этих функций следует, что гистерезисные свойства $\mathrm{XB}$, сильно выраженные для небольших чисел $n$, исчезают с возрастанием номера резонанса. Переход к безгистерезисным ХВ может быть немонотонным. Кроме того, при значениях амплитуд, соответствующих корням уравнения $J_{n}(b)=0$, резонанс с соответствующим номером $n$ отсутствует вовсе. Например, для главного резонанса так будет при значениях $A=v \omega_{0}^{2} I, \quad v \approx 3,9 ; 7,0 ; 10,2 ; \ldots$. Для второго резонанса $v \approx 5,2 ; 8,4 ; \ldots$. Таким образом, изменяя параметр $b$, мы можем получить длинную галерею ХВ. На рис. 1.10 изображен пример качественной картины ХВ неавтономного ротатора для больших амплитуд внешнего воздействия (субгармонические резонансы не показаны). 


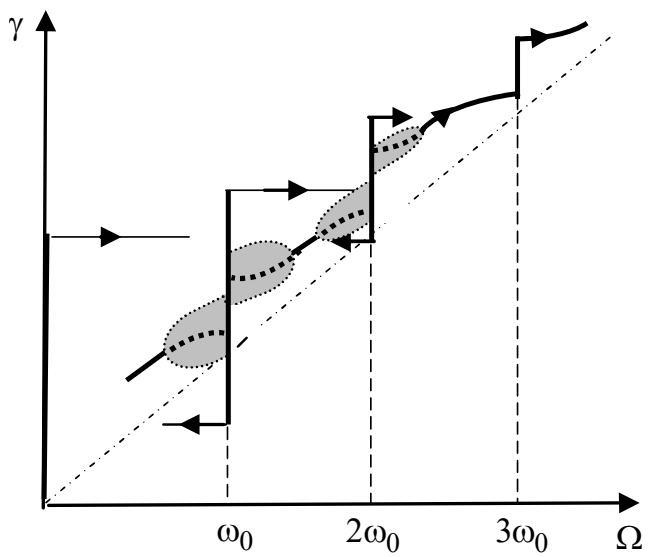

Рис. 1.10. ХВ неавтономного ротатора «чертова лестница» резонансов.

В рамках метода усреднения мы имеем общую картину динамических процессов. Это априорное знание превращает дальнейшее экспериментальное или численное исследование динамики системы в конструктивную работу; без знания общей картины гарантировано уподобление исследователя слепому котенку и утопление в море кажущихся бессвязанными фактов. В частности, при использовании полученной информации в качестве «затравочной» численное исследование может состоять в уточнении деталей самих процессов и их бифуркаций, в уточнении границ необходимых динамических режимов в пространстве параметров, в продолжении результатов на немалые значения параметра и т. д.

Численный эксперимент проводился для функций $F_{1}(\varphi) \equiv$ $\equiv 0, F_{2}(\varphi)=\sin \varphi, \quad f(\psi)=A \sin \psi \quad$ при $\quad$ значениях параметров $\mu=0,2, \quad A=3,5, \quad \omega_{0}=0,75$. Параметр $\gamma$ изменялся в интервале $[1,1177 ; 1,8248]$, захватывая две резонансные зоны. Для удобства система (1.8) записывалась в виде

$$
\begin{aligned}
& \dot{\varphi}=\gamma+x, \\
& \dot{x}=\mu(-x-\sin \varphi+A \sin \psi), \\
& \dot{\psi}=\omega_{0} .
\end{aligned}
$$


В галерее на рис. 1.11, кроме проекций траекторий системы (1.19) на развертку фазового цилиндра (сплошные линии), изображены также траектории точечного отображения Пуанкаре $(\varphi, \xi)_{\psi=\psi_{0}} \rightarrow(\bar{\varphi}, \bar{\xi})_{\psi=\psi_{0}+2 \pi}$, построенного для этой системы (точечные кривые).

Начиная с $\gamma=1,8248$ движемся вниз по этому параметру. Физически это соответствует уменьшению движущего момента маятника. При данном значении параметра движения маятника - квазипериодические вращения, которым соответствует эргодический тор. Динамический режим реализуется при любых начальных условиях (ГАУ). На рис. 1.11,а показан отрезок квазипериодической обмотки тора, а на рис. 1.11,б - инвариантная кривая точечного отображения (сравните с рис. 1.1,г). При уменьшении параметра и входе в серую зону (справа от второй ступени на рис. 1.10) на торе возникает субгармонический резонанс с числом вращения 11/5 (устойчивый и неустойчивый предельные циклы, рис. 1.11,в). Величина соответствующей резонансной ступени крайне мала (по этой причине подобные резонансы не «отлавливаются» методом усреднения). Далее циклы сближаются и исчезают. Образуется новый тор. В пространстве отображения Пуанкаре сценарий слияния циклов почти тот же самый, что и сценарий слияния состояний равновесия с рождением цикла от петли сепаратрисы седло-узла в фазовом пространстве автономного маятника. Разница в том, что перед слиянием циклов старый тор (как многообразие) теряет гладкость. При потере гладкости возможно рождение хаотического аттрактора. В нашем случае этот эффект настолько слаб, что фактически незаметен. По крайней мере, интервал его существования по параметру $\gamma$ крайне мал. На рис. 1.11,г изображена проекция этого тора, а на рис. 1.11,д - инвариантная кривая отображения. Темные места на рисунке - сгущения траекторий - являются «наследством» исчезнувшего цикла. Далее на образовавшемся торе возникает новый дробный резонанс, например, с числом вращения 15/7, который исчезает по тому же сценарию. Ситуация повторяется до тех пор, пока ротатор не совершает скачок с одного из таких дробных резонансов (в момент его исчезновения) на резонанс с числом вращения 2, т. е. в режим гармонической синхронизации (рис. $1.11, \mathrm{e})$. Скачок частоты вращения из субгармонического резо- 
нанса происходит вследствие крайне малой области притяжения предельного множества траекторий, образующегося после исчезновения предельного цикла. Малое вторжение в вычислительный процесс (изменение значения параметра в четвертом знаке) приводит к выходу системы из устойчивого состояния и скачкообразному переходу в режим гармонической синхронизации. Маложивущие по параметру динамические режимы не представляют физического интереса. В эксперименте величина второй гармонической ступени равна $\delta_{2}(\gamma)=0,32$. Для сравнения: $b=\frac{A}{\omega_{0} \sqrt{\omega_{0}^{2} I^{2}+1}}, \quad \delta_{2}(\gamma)=$ $=2\left|J_{2}(b)\right|=2 J_{2}(1,2024)=0,3198$.

При $\gamma=1,35$ ротатор скачком с резонансной ступени переходит в режим хаотических вращений. На картине ХВ (см. рис. 1.10) скачок происходит в левую половину серой зоны при второй ступени.

К этому моменту хаотический аттрактор является уже сложившимся. На рис. 1.11,ж изображен небольшой отрезок траектории, в противном случае оказался бы «замазанным» весь квадрат. В отображении Пуанкаре (рис. 1.11,3) наблюдается тор со «складкой и отростками» - признак хаотичности движения. Для уточнения характера бифуркаций, приводящих к хаосу, параметр $\gamma$ с малым шагом был увеличен. При этом было обнаружено, что хаотический аттрактор развивается по известному сценарию: субгармонический резонанс на торе $\rightarrow$ потеря тором гладкости $\rightarrow$ удвоение периода устойчивого резонансного цикла $\rightarrow$ странный аттрактор [23]. Его иногда называется хаотическим тор-аттрактором [26].

При дальнейшем уменьшении параметра $\gamma$ на хаотическом аттракторе (внутренняя бифуркация) рождается устойчивый предельный цикл - субгармонический резонанс с числом вращения 5/3 (рис. $1.11, и)$. Этот резонанс испытывает каскад удвоений периода с образованием аттрактора Фейгенбаума. На рис. 1.11,р изображен небольшой отрезок траектории, а на рис. 1.11,с - отображение Пуанкаре. В нижней части справа имеется неоднозначная ветвь. Далее к этому аттрактору подмешивается хаотический аттрактор, связанный с гомоклиническими траекториями седлового резонансного цикла (1/1). Происходит всплеск хаотичности движения ротатора (рис. $1.11, \mathrm{T,y})$. Далее происходит скачок частоты вращения ротато- 
ра на значение $\Omega=\omega_{0}=0,75$ - скачок в режим простой синхронизации. На рис. 1.11,ф зафиксирован первый резонанс со скачком при $\gamma=1,1177$.
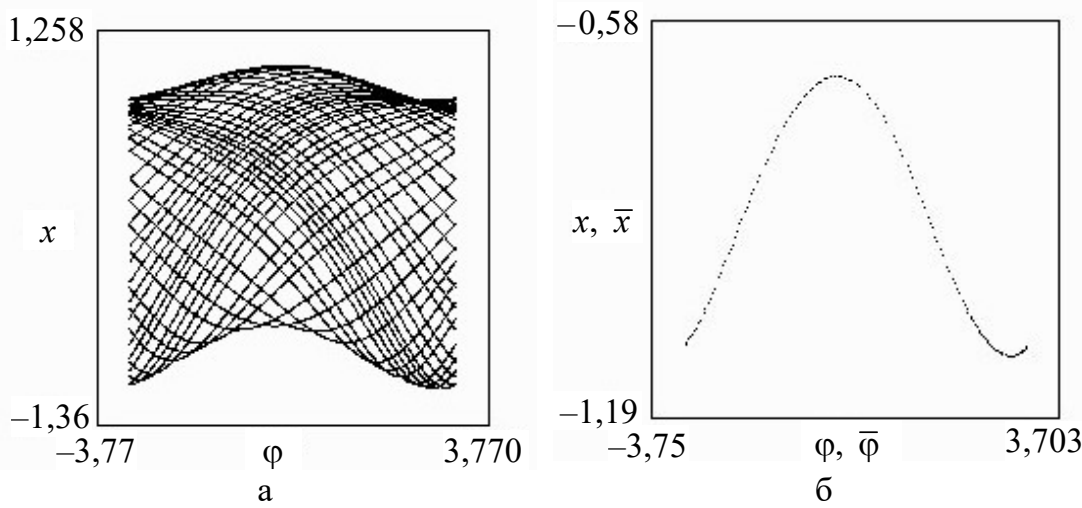

$$
\gamma=1,8248
$$
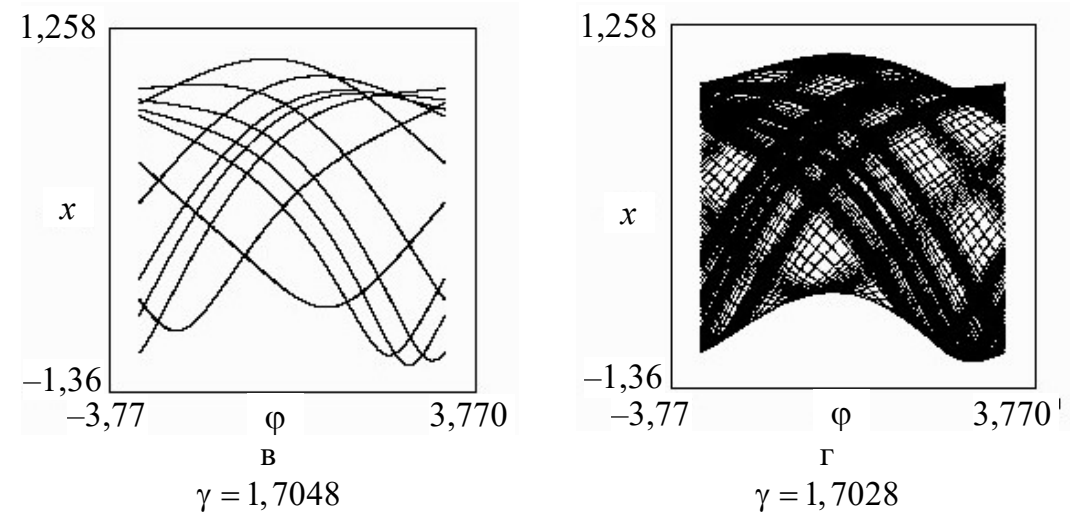

Рис. 1.11. Галерея фазовых портретов и портретов точечного отображения (см. также с. 35-37). 

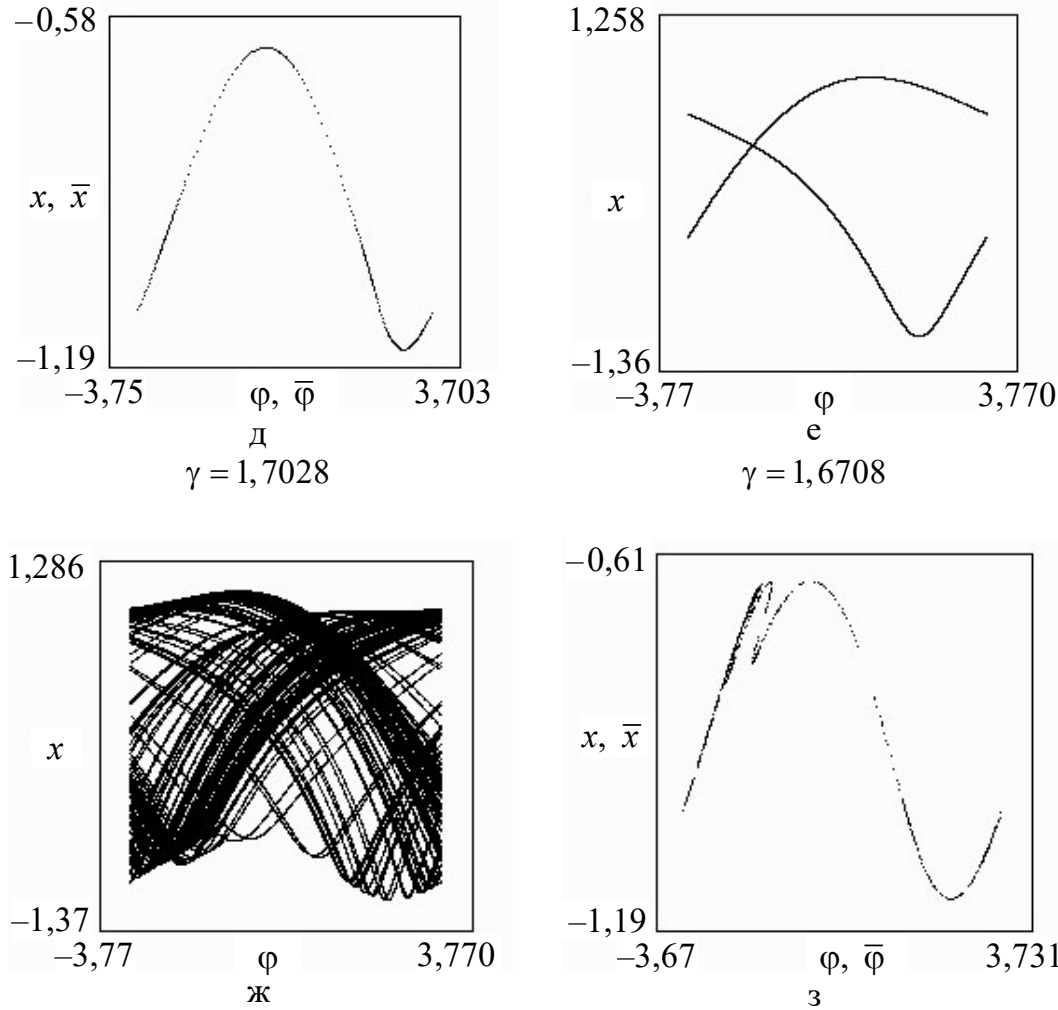

$$
\gamma=1,3518
$$

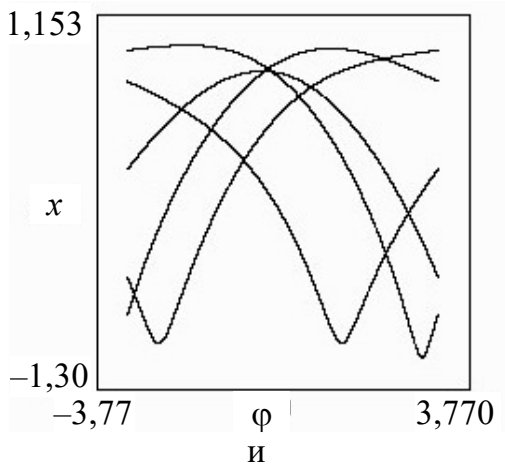

$\gamma=1,3128$

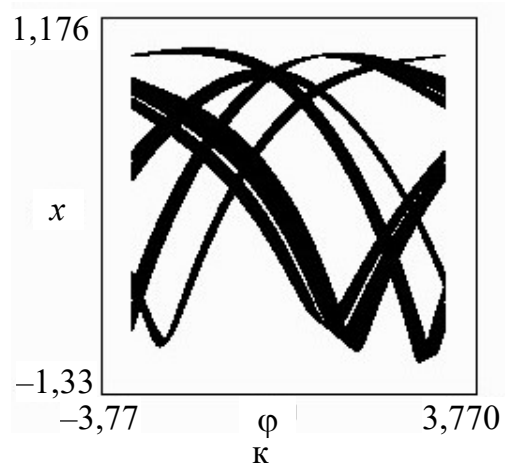

$\gamma=1,3058$

Рис. 1.11. Продолжение. 

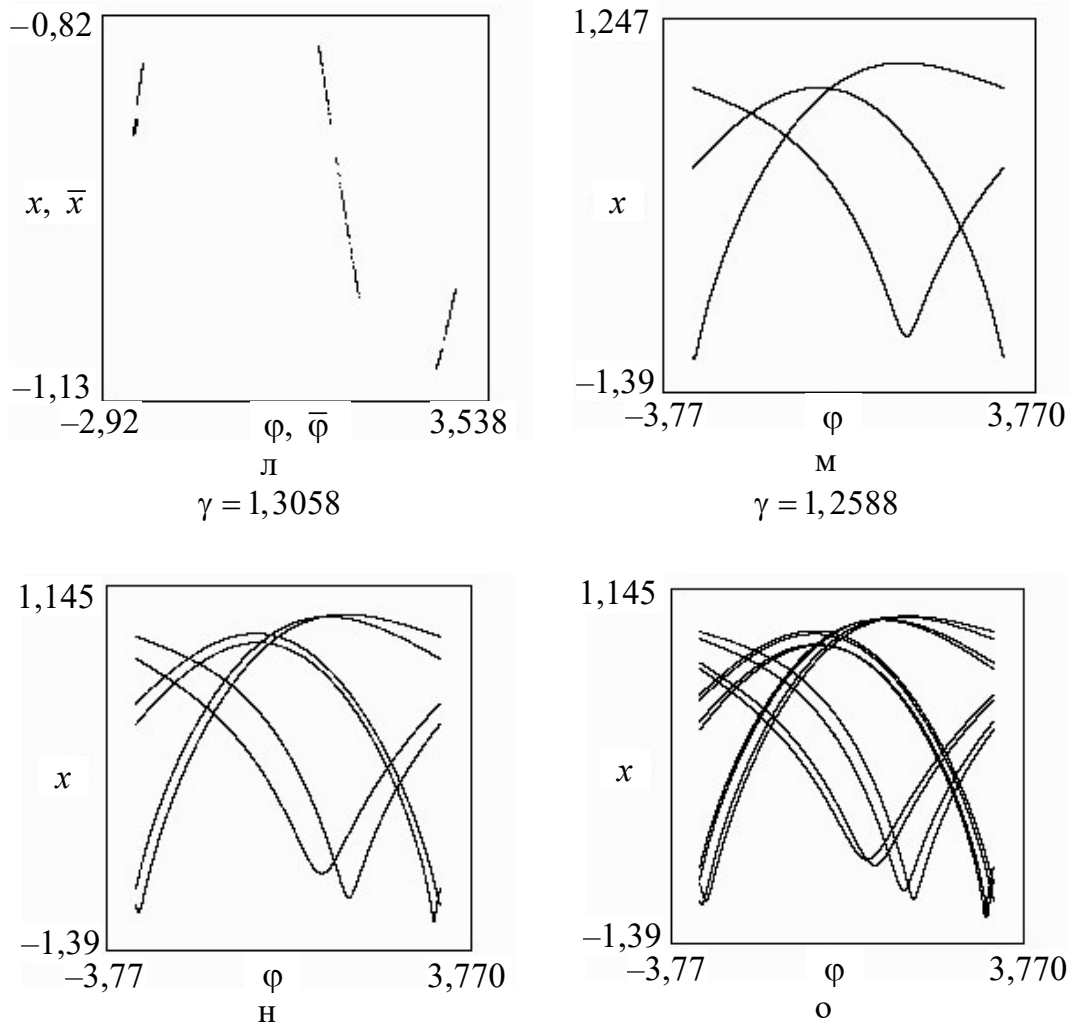

$\gamma=1,2447$

$\gamma=1,2387$

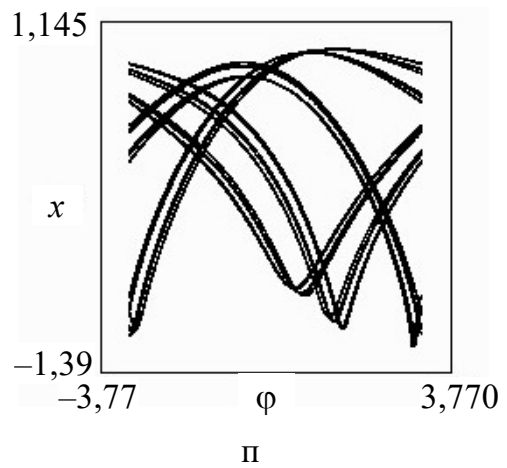

$\gamma=1,2377$

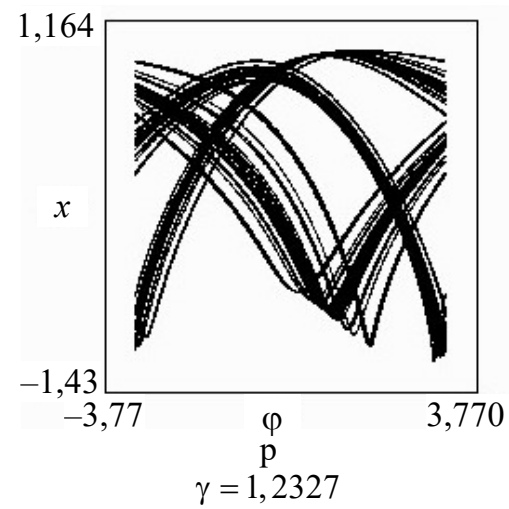

Рис. 1.11. Продолжение. 

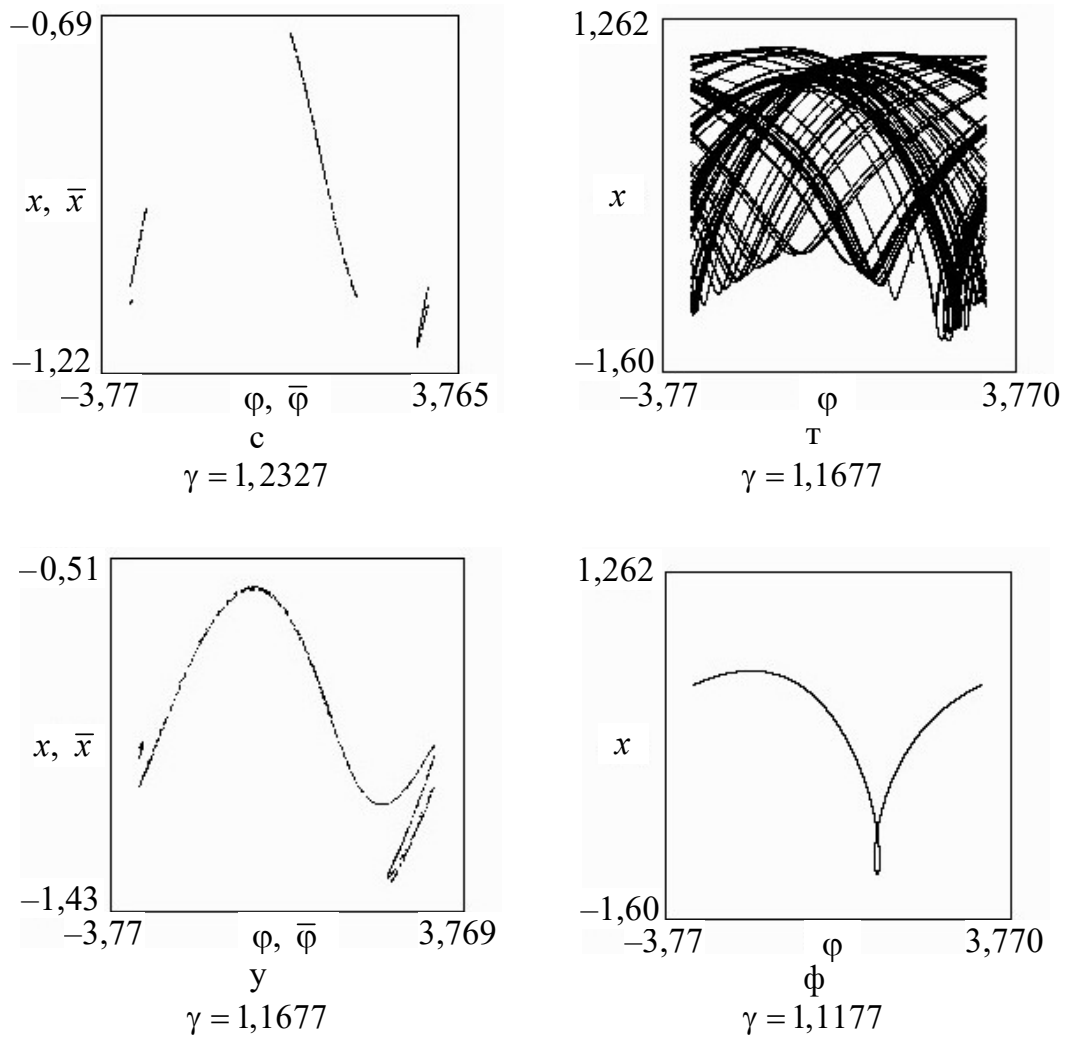

Рис. 1.11. Окончание.

Эффект рассеяния ХВ. При движении ротатора на странном аттракторе (см. рис. $1.11, \mathrm{T,y)}$ его средняя скорость $\left\langle\dot{\varphi}^{*}\left(t, t_{0}\right)\right\rangle_{t}=$ $=\omega_{0}+\left\langle\dot{\eta}^{*}\left(t, t_{0}\right)\right\rangle_{t}$ сильно зависит от начальных условий, т. е. тех фазовых координат, при которых ротатор входит в режим хаотических вращений, что, в принципе, определяется всей динамической предысторией ротатора. Естественно, что все это невозможно контролировать в любом реальном эксперименте. Поэтому при неоднократном повторении эксперимента прохождение режима хаотических вращений будет осуществляться по разным траекториям, имеющим разные средние. В результате получим неоднозначную $\mathrm{XB}$ с бесконечным множеством ветвей, имеющей форму веника. 
При этом возврат (скачок) в режим синхронизации будет происходить при различных значениях параметра $\gamma$. Эффект будет усиливаться конечностью времени усреднения и присутствием естественных шумов. Данный эффект, обнаруженный при исследовании динамики систем со сверхпроводящими переходами, устойчиво наблюдается в реальном эксперименте [27]. 


\section{АВТОНОМНЫЕ И НЕАВТОНОМНЫЕ СИСТЕМЫ С ПОЛУТОРА СТЕПЕНЯМИ СВОБОДЫ}

Интенсивные вибрации несбалансированных роторов являются причиной не только преждевременного износа дорогостоящего оборудования, но и возможных аварий с катастрофическими последствиями. Документальные свидетельства последствий таких аварий представлены в [28] и производят сильное впечатление. Кроме естественной разбалансировки роторов причинами повышенных вибраций могут стать и неверные инженерные решения. В частности, может случиться так, что внедренные дополнительные устройства виброгашения вместо положительного эффекта (и вопреки интуиции), напротив, приводят к усилению неустойчивости вращения роторов. Вообще говоря, устойчивость/неустойчивость систем - это область формального знания, и интуиция может оказаться плохим советчиком в решении связанных с устойчивостью проблем. Итог таков: нет другого пути, кроме как пройти по цепочке изучения динамики моделей с повышающимся числом степеней свободы, начиная с малого числа.

\section{1. Динамика ротатора с апериодической нагрузкой}

На рис. 2.1 приведена схема маятника Фруда (сечение), в муфту которого вложена другая муфта. Между ними существует вязкое трение. Вязкое трение существует также между муфтами и валом. Вал вращается с постоянной угловой скоростью. Момент силы трения, действующий на маятник со стороны вала, $\delta_{1}(\Omega-\dot{\varphi})$. Момент силы трения со стороны вложенной муфты $\delta_{2}(\omega-\dot{\varphi})$, где $\omega-$ ее мгновенная угловая частота. Момент силы тяжести равен $m g l \sin \varphi$. Момент силы трения, действующий на вложенную муфту со стороны вала, $\delta_{3}(\Omega-\omega)$, а со стороны муфты маятника 
$\delta_{2}(\dot{\varphi}-\omega)$. Параметры $\delta_{1}, \delta_{2}, \delta_{3}$ будем считать постоянными. Уравнения движения системы имеют вид

$$
\begin{aligned}
& I_{1} \ddot{\varphi}=\delta_{1}(\Omega-\dot{\varphi})+\delta_{2}(\omega-\dot{\varphi})-m g l \sin \varphi, \\
& I_{2} \dot{\omega}=\delta_{3}(\Omega-\omega)-\delta_{2}(\omega-\dot{\varphi}),
\end{aligned}
$$

где $I_{1}, I_{2}$ - приведенный момент инерции маятника и момент инерции вложенной муфты соответственно.

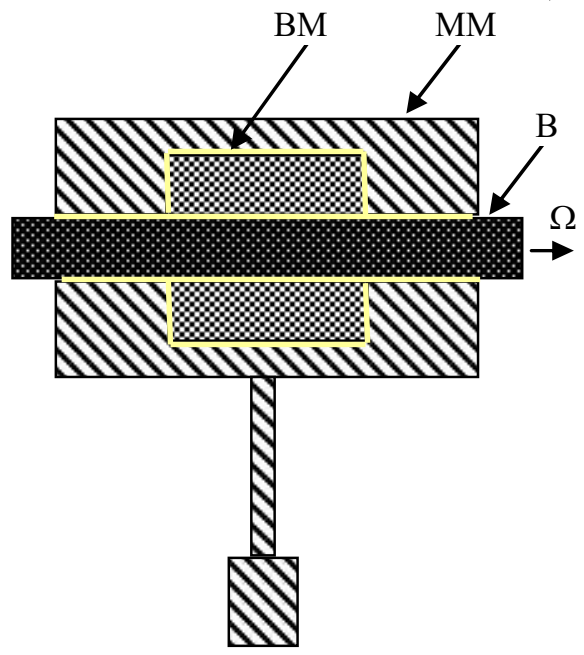

Рис. 2.1. Схема маятника Фруда (сечения): ММ - муфта маятника, ВМ - вложенная муфта, В - вал.

Эти уравнения преобразованием переменной $J=\frac{\delta_{2}}{m g l}(\omega-\dot{\varphi})-$ $-\frac{\delta_{2} \delta_{3}}{m g l\left(\delta_{2}+\delta_{3}\right)} \Omega$ и времени $\frac{m g l}{\delta_{1}} \tau=\tau_{n}$ приводятся к системе вида

$$
\begin{aligned}
& I \ddot{\varphi}+\dot{\varphi}+\sin \varphi=\gamma+J, \\
& \dot{J}+h J=a \dot{\varphi}+b(\sin \varphi-\gamma),
\end{aligned}
$$


где $I=\frac{I_{1} m g l}{\delta_{1}^{2}}, \gamma=\frac{\Omega}{m g l}\left(\frac{\delta_{1} \delta_{2}+\delta_{1} \delta_{3}+\delta_{2} \delta_{3}}{\delta_{2}+\delta_{3}}\right), \quad h=\frac{\delta_{1}}{m g l}\left(\frac{\delta_{1}+\delta_{2}}{I_{2}}+\frac{\delta_{2}}{I_{1}}\right)$, $a=\frac{\delta_{2}}{m g l}\left(\frac{\delta_{1}}{I_{1}}-\frac{\delta_{3}}{I_{2}}\right), \quad b=\frac{\delta_{1} \delta_{2}}{I_{1} m g l}$.

С целью популяризации модели (2.1) рассмотрим еще одну систему, схематично изображенную на рис. 2.2. Асинхронный электродвигатель ограниченной мощности нагружен жестким валом с жестко посаженным на него несбалансированным диском. На вал посажена инерционная муфта. Между валом и муфтой имеется вязкое трение. Вдоль вала муфта не перемещается.

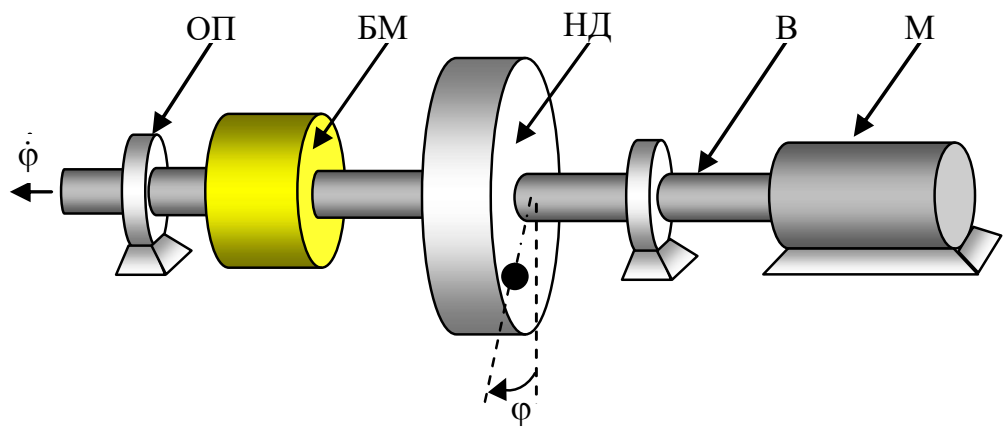

Рис. 2.2. Асинхронный мотор с несбалансированным диском и балансирующей муфтой: М - асинхронный мотор, В - вал, НД - несбалансированный диск, БМ - балансирующая муфта,

ОП - опорный подшипник.

Стабилизирующие свойства инерционной муфты физически вроде бы прозрачны: при отклонении рабочей частоты вверх от номинала муфта поглощает энергию двигателя, а при отклонении вниз часть ее кинетической энергии передается валу, т. е. инерционная муфта играет роль интегрирующего (усредняющего) звена или, иначе говоря, частотного фильтра (в радиотехнической терминологии).

Будем считать, что движущий момент двигателя является линейной функцией вида $M_{d}=M_{0}-\lambda \dot{\varphi}$, где $M_{0}=$ const, $\lambda \dot{\varphi}-$ приведенный момент сил трения. Моменты сил, действующих на ротор двигателя: $m g \varepsilon \sin \varphi$ - момент силы тяжести массы дисбаланса 
$(\varepsilon-$ эксцентриситет), $\delta(\omega-\dot{\varphi})-$ момент сил вязкого трения со стороны муфты. Момент силы, действующей на муфту со стороны вала, $\delta(\omega-\dot{\varphi})$. При этом уравнения движения системы имеют вид

$$
\begin{aligned}
& I_{1} \ddot{\varphi}=M_{0}-\lambda \dot{\varphi}+\delta(\omega-\dot{\varphi})-m g \varepsilon \sin \varphi, \\
& I_{2} \dot{\omega}=-\delta(\omega-\dot{\varphi}),
\end{aligned}
$$

где $I_{1}, I_{2}$ - приведенный момент инерции ротора двигателя и момент инерции муфты соответственно; $m$ - дисбаланс; $\varepsilon-$ эксцентриситет; $\lambda, \delta$ - коэффициенты вязкого трения.

В результате преобразования переменной $\frac{\delta}{m g \varepsilon} \omega=J$ и времени $\frac{m g \varepsilon}{\lambda+\delta} \tau=\tau_{n}$ уравнения (2.2) сводятся к системе (2.1) с параметрами: $I=\frac{I_{1} m g \varepsilon}{(\lambda+\delta)^{2}}, \quad \gamma=\frac{M_{0}}{m g \varepsilon}, h=\frac{\delta(\lambda+\delta)}{I_{2} m g \varepsilon}, a=\frac{\delta^{2}}{I_{2} m g \varepsilon}$.

Рассмотрим еще одну систему. На рис. 2.3, а изображена конструкция сверхпроводящего квантового интерферометра Р-СКВИД (RSQUID), применяющегося для измерения сверхмалых магнитных полей [5]. На рис. 2.3,б приведена его эквивалентная электрическая схема.

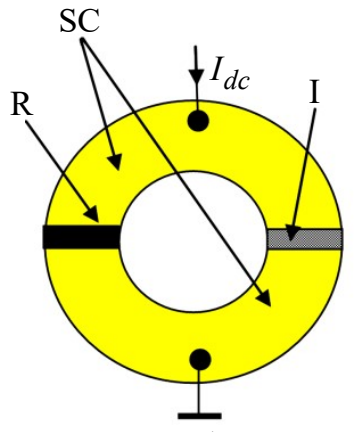

a

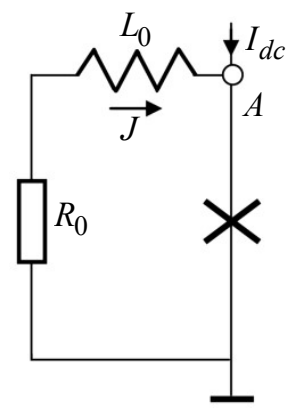

B

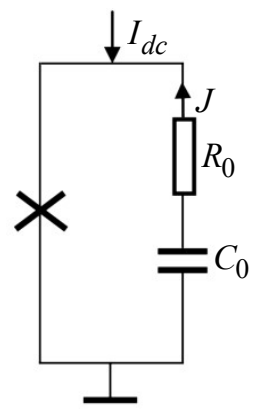

Рис. 2.3. Сверхпроводящий квантовый интерферометр Р-СКВИД:

$\mathrm{a}$ - конструкция ( $\mathrm{R}$ - резистор, $\mathrm{SC}$ - сверхпроводники, I - изолятор); б - эквивалентная электрическая схема; в - сверхпроводящий переход, нагруженный $R C$-цепочкой. 
Динамическую модель системы получаем из законов Кирхгоффа и резистивной модели перехода. В безразмерных переменных параметрах и времени она имеет следующий вид:

$$
\begin{aligned}
& C \ddot{\varphi}+\dot{\varphi}+\sin \varphi=\gamma+J, \\
& j+h J=a \dot{\varphi},
\end{aligned}
$$

где $\frac{I_{d c}}{I_{c}}=\gamma, \frac{r}{l}=h,-\frac{1}{l}=a ; \quad r, l-$ безразмерные сопротивление и индуктивность кольца, $C$ - емкость перехода.

Первое уравнение получается из уравнения для токов узла $A$. Второе уравнение отражает равенство напряжений на $R L$-цепи и сверхпроводящем переходе. Легко видеть, что это та же самая модель.

Можно убедиться, что динамика схемы на рис. 2.3,в также описывается моделью (2.1).

Как видим, уравнения перечисленных систем с точностью до обозначений и физического смысла переменных представляются одной и той же моделью, поэтому она заслуживает внимания.

Обратимся к динамическим свойствам системы (2.1). Система имеет два состояния равновесия $O\left(\varphi_{0}, \dot{\varphi}_{0}, J_{0}\right): O_{1}(\arcsin \gamma, 0,0)$ и $O_{2}(\pi-\arcsin \gamma, 0,0)$. Состояние равновесия $O_{1}$ устойчиво во всей области существования $|\gamma|<1$ и является либо узлом, либо фокусом в зависимости от значений параметров системы. Состояние равновесия $\mathrm{O}_{2}$ в зависимости от параметров является либо седло-узлом, либо седло-фокусом. $\operatorname{dim} W^{s}=2, \operatorname{dim} W^{u}=1$, где $W^{s}, W^{u}$ - устойчивое и неустойчивое многообразия состояния равновесия $\mathrm{O}_{2}$.

Периодические траектории колебательного типа (ограниченные по $\varphi)$ в фазовом пространстве системы $G(\varphi, \dot{\varphi}, J)$ отсутствуют. В частности, при выполнении неравенства $I b(h-a)+b-a>0$ для рассматриваемых физических систем этот факт устанавливается при помощи периодической функции Ляпунова: 


$$
\begin{aligned}
V & =\frac{I b(h-a)+b-a}{2} I \dot{\varphi}^{2}+\frac{1}{2} J^{2}+b I \dot{\varphi} J+ \\
& +\left(b+b h I-a-b^{2} I\right) \int_{\varphi_{0}}^{\varphi}(\sin \varphi-\gamma) d \varphi .
\end{aligned}
$$

Ее производная, вычисленная вдоль траекторий системы (2.1), $\dot{V}=-(I b(h-a)+b-a) \dot{\varphi}^{2}-(h-b) J^{2} \leq 0 \quad$ является отрицательной во всем фазовом пространстве.

Таким образом, в рассматриваемой динамической системе не могут существовать какие-либо стационарные движения колебательного типа (колебательные предельные циклы).

Перекладывая изложенную информацию на приведенные выше физические системы, скажем, что при вариации начальных условий маятник Фруда или несбалансированный диск (во второй системе) со временем оказываются или в состоянии равновесия, или во вращательном движении. Соответственно, джозефсоновский переход со временем приходит или в сверхпроводящее состояние без какихлибо дополнительных осцилляций электромагнитного поля, или в режим генерации.

Рассмотрим структуры вращательных траекторий подробнее. Исключением переменной $J$ и преобразованием времени система (2.1) сводится к эквивалентному уравнению третьего порядка

$$
\beta \dddot{\varphi}+\ddot{\varphi}+(\alpha \cos \varphi+\lambda) \dot{\varphi}+\sin \varphi=\gamma,
$$

где $\beta=\sqrt{\frac{I^{2} h}{(1+h I)^{3}}}, \alpha=\frac{1}{\sqrt{h(1+h I)}}, \lambda=\frac{h-a}{\sqrt{h(1+h I)}}$.

Уравнение (2.3) известно из теории систем синхронизации [29]. Его свойства принципиально зависят от соотношения $\alpha / \beta$. Нетрудно выяснить, что в интересующих нас системах всегда выполняется неравенство $\alpha>\beta$. При этом условии возможны два случая: в первом динамика системы является регулярной для всех значений параметров, при этом бифуркационная диаграмма в плоскости $(\gamma, \lambda), \alpha=$ const, $\beta=$ const, имеет вид, изображенный на рис. 2.4; во втором случае на бифуркационной диаграмме имеется область 
параметров, для которых система имеет хаотическую динамику (рис. 2.5).

Обсудим первый случай. На бифуркационной диаграмме кривая $\lambda=\lambda^{0}(\gamma)$ соответствует двойному (кратному) вращательному предельному циклу, рождающемуся от «сгущения» фазовых траекторий. В момент рождения цикл имеет частоту вращения $\Omega>0$. При переходе параметров из области (1) в область (4) двойной цикл разделяется на два: устойчивый и неустойчивый. Двумерный аналог фазового портрета уравнения (2.3) на развертке фазового цилиндра изображен на рис. 2.4,б. При увеличении параметра $\gamma$ устойчивый цикл движется вверх по цилиндру, а неустойчивый вниз. Для параметров на кривой Трикоми $\lambda=\lambda^{*}(\gamma)$ и $\lambda<\lambda_{0}$ неустойчивый цикл «влипает» в петлю сепаратрисы $O_{2}$ и исчезает. При обратном движении параметра $\gamma$ устойчивый цикл движется вниз. На кривой Трикоми от петли рождается неустойчивый цикл, который далее движется навстречу устойчивому. На кривой $\lambda=\lambda^{0}(\gamma)$ циклы «слипаются» и исчезают. В области (1) состояние равновесия $O_{1}$ устойчиво глобально. При значениях $\lambda_{0}<\lambda<\lambda_{*}$ от петли сепаратрисы седлового состояния равновесия рождается устойчивый предельный цикл с нулевой частотой вращения, которая

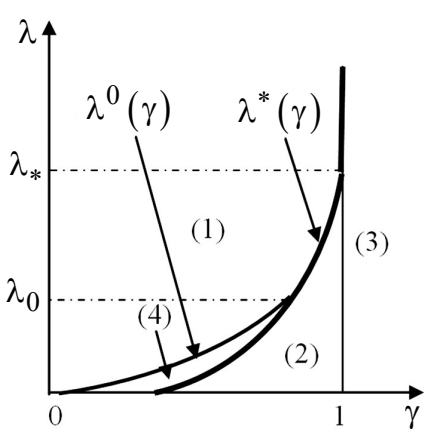

a

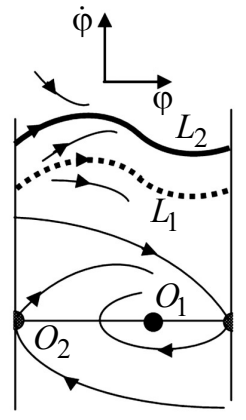

6

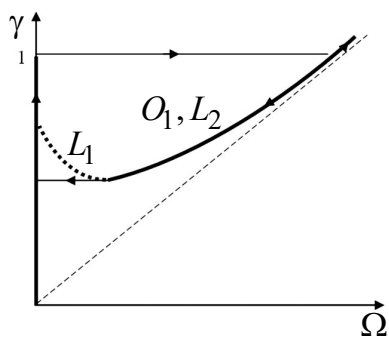

B

Рис. 2.4. Бифуркационная диаграмма в случае регулярной динамики (a); двумерный аналог фазового портрета системы для области параметров (4) (б); качественная картина ХВ ротатора при $\lambda<\lambda_{0}$ (в). 
затем увеличивается с ростом $\gamma$. Двумерные аналоги фазовых портретов уравнения (2.3) для областей параметров (1), (2) и (3) изображены на рис. 1.1,б-г соответственно. Как всегда, качественные картины ХВ ротатора строим, используя фазовые портреты, бифуркации и поведение предельных траекторий системы. Параметр $\gamma$ изменяем вдоль прямой $\lambda=$ const. При любом $\lambda=$ const из интервала $\lambda>\lambda_{*} \mathrm{XB}$ качественно совпадает с XВ автономного ротатора (рис. 2.4,a). Качественное совпадение ХВ имеется и для любого $\lambda$ из интервала $\lambda_{0}<\lambda<\lambda_{*}$ (рис. 2.4,б). При значениях параметра $\lambda$ из интервала $\lambda<\lambda_{0}$ получаем качественную картину XB, изображенную на рис. 2.4,в. Поясним: параметр $\gamma$ изменяем квазистатически, начиная с малых значений; если он находится в области (1), то при любом начальном условии ротатор приходит в устойчивое состояние равновесия $O_{1}$ (см. рис. 1.1, a) и находится в нем вплоть до его исчезновения при $\gamma=1+0$, «не замечая» происходящих бифуркаций и перестроек фазовых портретов. Этим реализуется нулевая ступень. При значении $\gamma=1+0$ ротатор скачком переходит во вращательный режим (см. рис. 1.1,г). Далее частота цикла увеличивается, а вращательная ветвь ХВ возрастает, приближаясь к асимптоте - биссектрисе угла. При квазистатическом изменении параметра $\gamma$ вниз ротатор «находится» на предельном цикле, до времени «не замечая» перестроек окружающего пространства, в частности того, что при значении $\gamma$, соответствующем кривой Трикоми, рождается «убийца» вращений - неустойчивый предельный цикл, который движется навстречу устойчивому. При значении $\gamma$ на кривой $\lambda^{0}(\gamma)$ циклы сближаются и исчезают. Ротатор скачком возвращается на нулевую ступень.

Обсудим второй случай. Здесь на бифуркационной диаграмме добавилась «серая» область (5) (см. рис. 2.5,a), соответствующая хаотической динамике системы. Поясним ситуацию. Допустим, что параметры $\gamma, \lambda$ изменяются вдоль кривой Трикоми в сторону ее возрастания. Тогда для $\lambda<\lambda_{1}$ от петли сепаратрисы $O_{2}$ рождается единственный неустойчивый цикл (переход из (2) в (4)). При этом так называемая седловая величина $\sigma_{s}>0$ [30]. Для $\lambda>\lambda_{2}$ в петлю влипает устойчивый цикл (переход из (2) в (1)), при этом $\sigma_{s}<0$, 
т. е. имеет место смена знака седловой величины. Кроме того, при движении вдоль кривой на двумерном устойчивом многообразии $W^{S}$ состояния равновесия $O_{2}$ имеет место бифуркация рождения фокуса из узла, и в момент образования петли седло-фокусная величина $\sigma_{s f}>0$. Таким образом, приходим к выводу о выполнении условий теоремы Л. П. Шильникова о существовании в окрестности петли седло-фокуса счетного множества периодических движений седлового типа - «спирального» хаотического аттрактора Шильникова (подробнее см. [30]). Следует заметить, что кроме петли седло-фокуса при изменении параметров в области (5) могут наблюдаться и другие бифуркации, в частности цепочки удвоений периода предельного цикла с последующим рождением аттрактора Фейгенбаума, а также объединение хаотических аттракторов («кризис аттракторов» или «перемежаемость хаосов»). На подробностях остановимся при обсуждении результатов численного эксперимента.
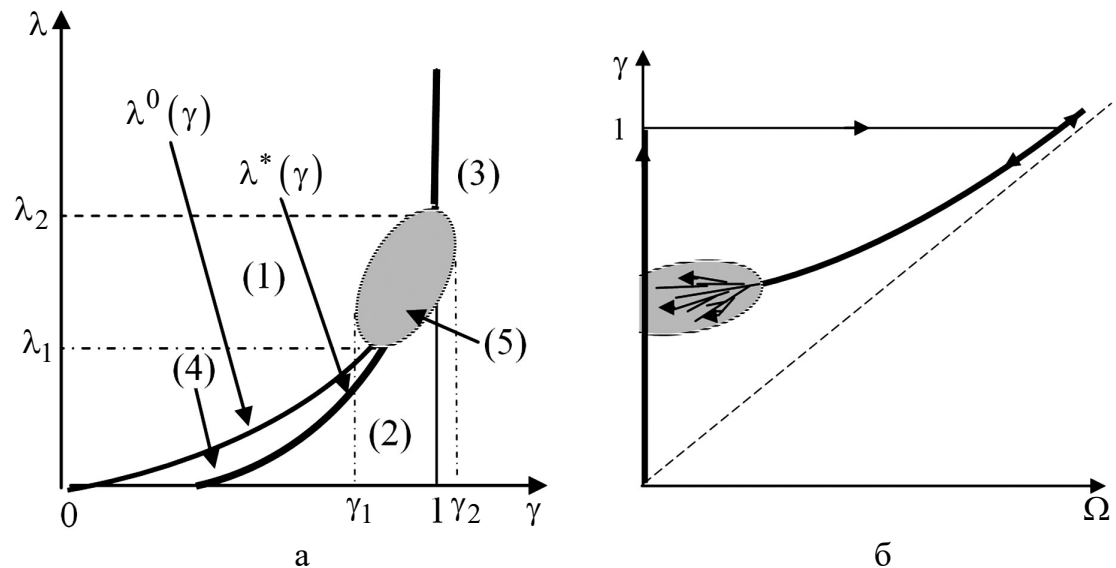

6

Рис. 2.5. Бифуркационная диаграмма в случае существования динамического хаоса (a); ХВ ротатора с эффектом рассеяния,

$$
\lambda_{1}<\lambda<\lambda_{2} \text { (б). }
$$

Численный эксперимент. Опишем динамику системы при изменении параметров в серой зоне. Уменьшаем постоянную составляющую движущего момента ротатора - параметра $\gamma$ - при посто- 
янстве других параметров системы: $\mu=0,08, \quad h=0,05524$, $a=-0,0809, b=0,021$. При этих параметрах $\lambda=0,567$.

На рис. 2.6 представлены фазовые портреты и портреты точечного отображения $(x, J)_{\varphi=\varphi_{0}} \rightarrow(\bar{x}, \bar{J})_{\varphi=\varphi_{0}+2 \pi}$, построенные для эквивалентной динамической системы:

$$
\begin{aligned}
& \dot{\varphi}=\gamma+x, \\
& \dot{x}=\mu(-x-\sin \varphi+J), \\
& \dot{J}=-h J+a(\gamma+x)+b(\sin \varphi-\gamma) .
\end{aligned}
$$

При $\gamma=1,29$ (и $\gamma>1,29$ ) ротатор совершает периодические вращения (рис. 2.6,a). При $\gamma=1,282$ предельный цикл совершает первую бифуркацию удвоения периода (рис. 2.6,б). Далее следует каскад удвоений (рис. 2.6,в-е), заканчивающийся аттрактором Фейгенбаума (рис. 2.6,ж). На рис. 2.6,3 изображена кривая отображения, соответствующая этому странному аттрактору. Вид кривой подсказывает, что подходящим выбором координат это отображение преобразуется к классическому отображению параболы. При уменьшении $\gamma$ на хаотическом аттракторе рождается устойчивый вращательно-колебательный предельный цикл утроенного периода (главный период $2 \pi$ ). Характерной особенностью этого цикла является поворот фазы на двумерном устойчивом многообразии. Движение на цикле происходит так, как если бы в фазовом пространстве системы существовало упоминавшееся выше состояние равновесия седло-фокус с двумерным устойчивым и одномерным неустойчивым многообразиями. Можно сказать, что этот предельный цикл является «наследством» аттрактора Шильникова. При уменьшении $\gamma$ этот предельный цикл теряет устойчивость, и вместо него рождается устойчивый пятикратный цикл. Далее ситуация повторяется, что в конечном итоге приводит к хаотическому движению ротатора на объединенном хаотическом аттракторе Шильникова - Фейгенбаума. При объединении аттракторов происходит всплеск значений характеристик хаотического движения (максимального ляпуновского показателя, размерности, пьедестала спектра и др.) по отношению к соответствующим характеристикам движения на отдельном аттракторе. Отображение Пуанкаре, соответствующее движению на объединенном хаотическом аттракторе, показано на рис. 2.6,м. 
При дальнейшем уменьшении $\gamma$ на хаотическом аттракторе рождается устойчивый предельный цикл двойного периода (рис. 2.6,н), а затем ротатор из режима вращений скачком переходит в состояние равновесия. Так происходит в случае параметров, заданных в эксперименте. Для других параметров вырождение хаоса происходит через бифуркацию петли сепаратрисы седло-фокуса. Заметим, что проследить бифуркационный сценарий достаточно трудно в силу узости соответствующей области параметров. Заметим также, что описанный нами механизм хаотизации динамических процессов устойчиво наблюдается в динамике квантовых интерферометров [5].

Рассмотренная система - один из примеров того, насколько может усложниться динамика автономного ротатора при подключении к нему, казалось бы, безобидных элементов вроде балансирующей муфты.

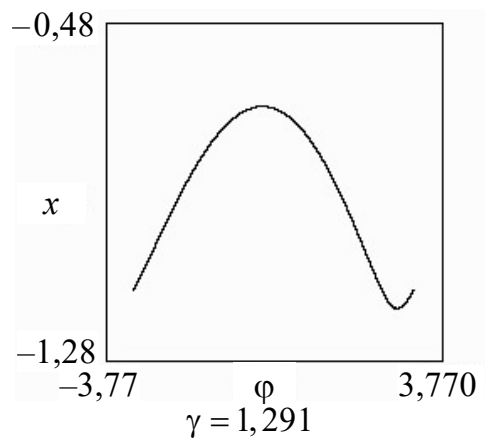

a

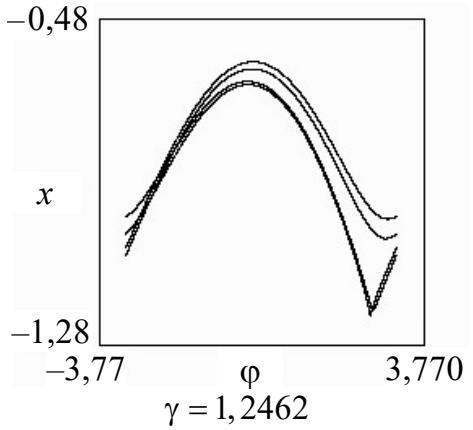

B

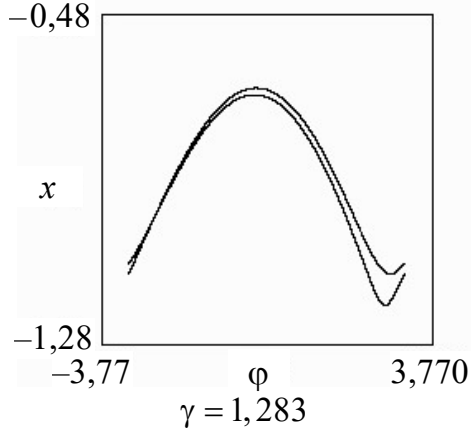

6

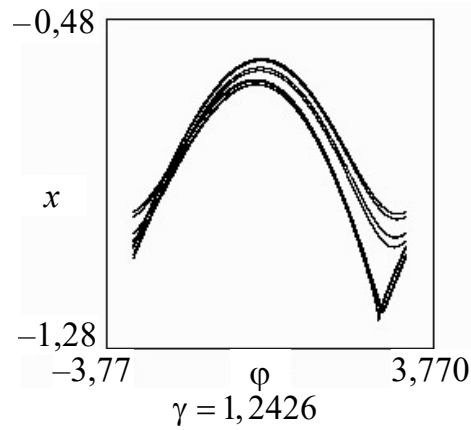

$\Gamma$

Рис. 2.6. Галерея фазовых портретов и портретов точечного отображения (см. также с. 50-51). 

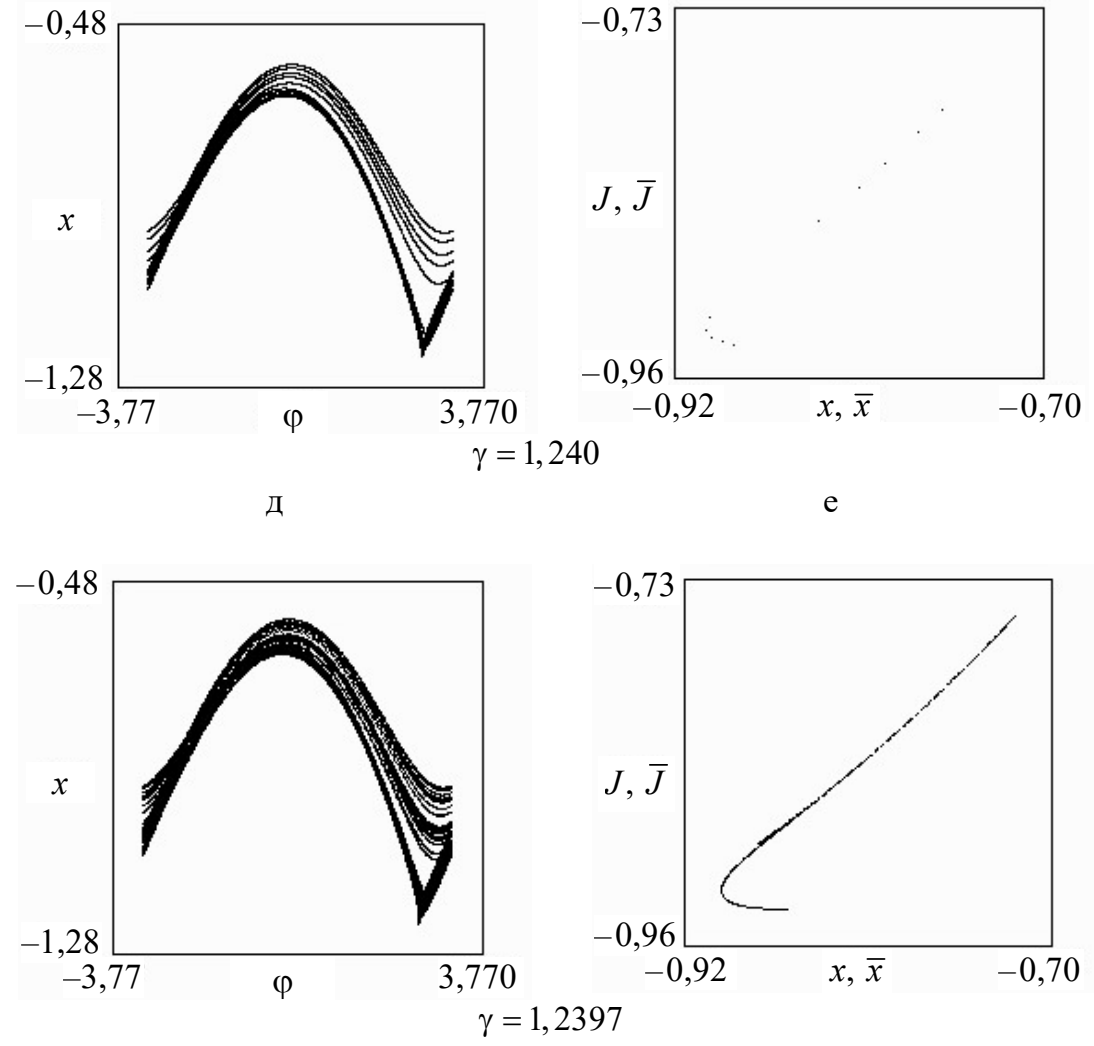

ж

3
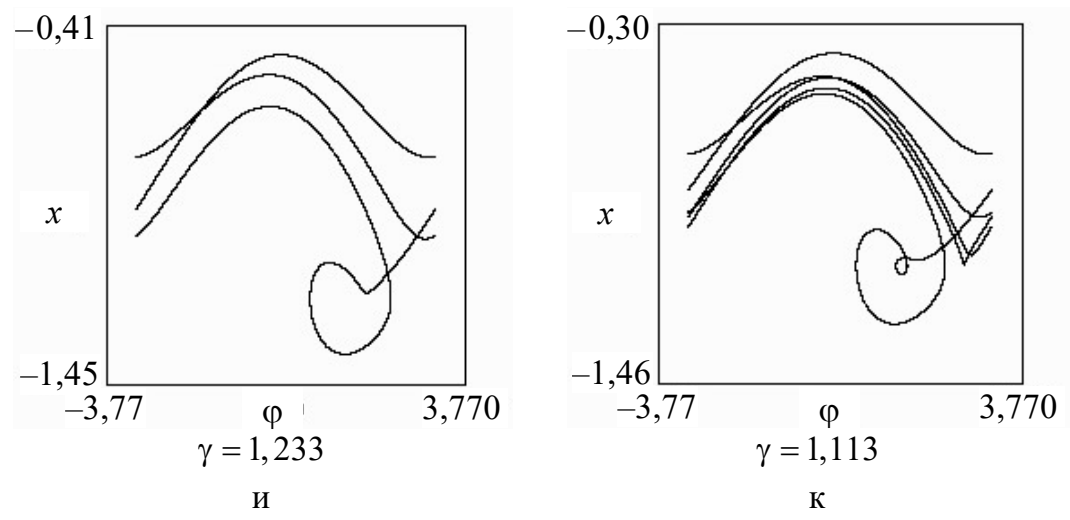

Рис. 2.6. Продолжение. 


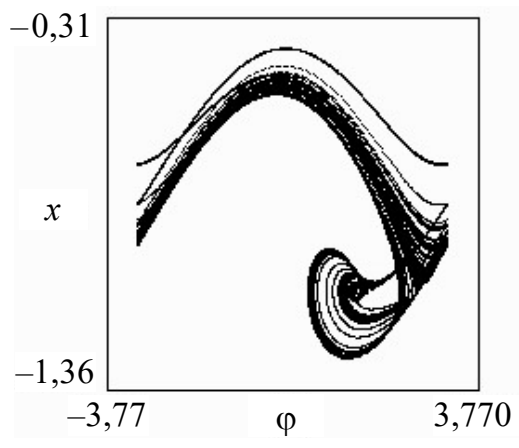

$\gamma=1,1095$

л

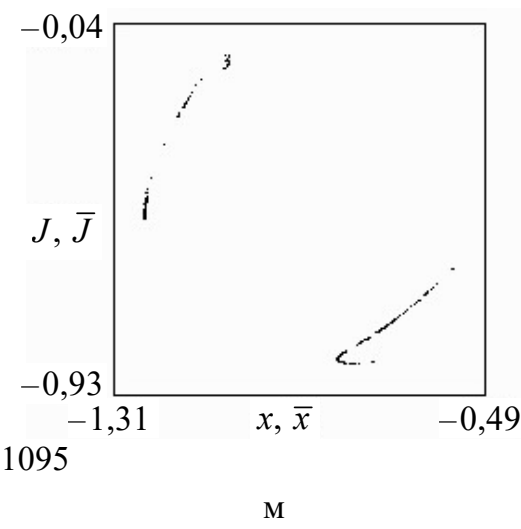

M

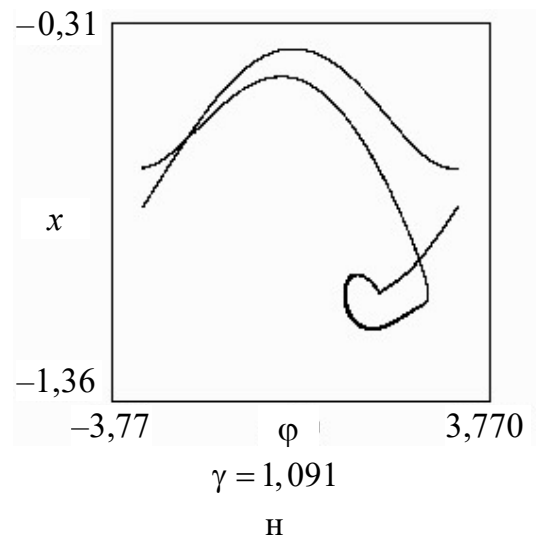

Рис. 2.6. Окончание.

\section{2. Синхронизация и динамический хаос в системе неавтономного ротатора с апериодической нагрузкой}

Снова обратимся к маятнику Фруда с балансирующей муфтой (см. рис. 2.1,a). Будем считать теперь, что вал маятника вращается по закону $\Omega(t)=\Omega_{0}+\Omega_{s} \sin \psi, \dot{\psi}=\varpi_{0}$, при этом уравнения движения системы имеют вид: 


$$
\begin{aligned}
& I_{1} \ddot{\varphi}=\delta_{1}\left(\Omega_{0}-\dot{\varphi}\right)+\delta_{2}(\omega-\dot{\varphi})-m g l \sin \varphi+\delta_{1} \Omega_{s} \sin \psi, \\
& I_{2} \dot{\omega}=\delta_{3}\left(\Omega_{0}-\omega\right)-\delta_{2}(\omega-\dot{\varphi})+\delta_{3} \Omega_{s} \sin \psi, \\
& \dot{\psi}=\varpi_{0} .
\end{aligned}
$$

Выполним цепочку преобразований уравнений (2.4), сводя их к виду, аналогичному автономному случаю. Во-первых, удалим периодическую функцию из второго уравнения заменой $\omega=A_{s} \sin \psi+A_{c} \cos \psi+y, \quad$ где $\quad A_{s}=\frac{\delta_{3}\left(\delta_{2}+\delta_{3}\right)}{\left(I_{2} \varpi_{0}\right)^{2}+\left(\delta_{2}+\delta_{3}\right)^{2}} \Omega_{s}$, $A_{c}=-\frac{I_{2} \delta_{3} \varpi_{0}}{\left(I_{2} \varpi_{0}\right)^{2}+\left(\delta_{2}+\delta_{3}\right)^{2}} \Omega_{s}$. В результате получим следующую систему:

$$
\begin{aligned}
& I_{1} \ddot{\varphi}+\delta_{1} \dot{\varphi}+m g l \sin \varphi=\delta_{1} \Omega_{0}+\delta_{2}(y-\dot{\varphi}) y+B \sin \left(\psi+\psi_{0}\right), \\
& I_{2}(y-\dot{\varphi})+\left(\delta_{2}+\delta_{3}\right)(y-\dot{\varphi})=\delta_{3} \Omega_{0}-\delta_{3} \dot{\varphi}-I_{2} \ddot{\varphi}, \\
& \dot{\psi}=\varpi_{0},
\end{aligned}
$$

где $B=\sqrt{\left(\delta_{2} A_{s}+\delta_{1} \Omega_{s}\right)^{2}+\left(\delta_{2} A_{c}\right)^{2}}, \quad \psi_{0}=\operatorname{arctg} \frac{\delta_{2} A_{c}}{\delta_{2} A_{s}+\delta_{1} \Omega_{s}}$.

Далее, как и в автономном случае, преобразуем в (2.5) время $\frac{m g l}{\delta_{1}} \tau=\tau_{n} \quad$ и $\quad$ переменные $\quad J=\frac{\delta_{2}}{m g l}(y-\dot{\varphi})-\frac{\delta_{2} \delta_{3}}{m g l\left(\delta_{2}+\delta_{3}\right)} \Omega_{0}$, $\psi+\psi_{0}=\psi_{n}$. В результате модель приобретает окончательный вид:

$$
\begin{aligned}
& I \ddot{\varphi}+\dot{\varphi}+\sin \varphi=\gamma+J+A \sin \psi, \\
& \dot{J}+h J=a \dot{\varphi}+b(\sin \varphi-\gamma-A \sin \psi), \\
& \dot{\psi}=\omega_{0},
\end{aligned}
$$

где $A=\frac{B}{m g l}, \quad \omega_{0}=\frac{\delta_{1}}{m g l} \varpi_{0}$, а другие параметры те же, что и в автономном случае (2.1). Индекс «n» в уравнениях опущен.

Рассматриваем динамику системы (2.6) в различных асимптотических случаях значений параметра $I$. 
Асимптотика $I<<1$. В этом случае, согласно $[15,16]$, сингулярно возмущенная система (2.6) имеет в фазовом пространстве $G(\varphi, \psi, \dot{\varphi}, J)$ устойчивую интегральную поверхность «медленных движений». Интегральные кривые на этой поверхности являются траекториями неавтономного ротатора. Его параметры близки $(\sim I)$ к тем, что получаются из системы (2.6) при $I=0$. В результате преобразований порождающая система сводится к уравнению неавтономного ротатора $(1.8)$ с функциями $F_{1}(\varphi)=\varepsilon \cos \varphi$, $F_{2}(\varphi)=\sin \varphi$. При этом роль параметров $I, \varepsilon, \gamma, A, \omega_{0}$ уравнения (1.8) играют комбинации параметров системы (2.6): $\frac{h-b}{(h-a)^{2}}, \frac{1}{(h-a)}$, $\gamma, \frac{\sqrt{\omega_{0}^{2}+(h-b)^{2}}}{h-b} A, \frac{h-a}{h-b} \omega_{0}$ соответственно. Считаем, что этот случай достаточно исследован в разделе 1.2 .

Асимптотика $I^{-1}=\mu<<1$. В этом случае преобразуем уравнения (2.6) к системе в стандартной форме с особенностями, изложенными в Приложении I. Рассматриваем область параметров

$$
D_{\mu}=\left\{I^{-1}=\mu, h=\mu h^{*}, a=\mu a^{*}, b=\mu b^{*}, \frac{h-a}{h-b}\left(\frac{h-b}{h-a} \gamma-\omega_{0}\right)=\mu \Delta\right\} .
$$

Физически данная область соответствует зоне простой принудительной синхронизации квазилинейного ротатора с апериодической нагрузкой, имеющей малое затухание.

Выполним в (2.6) замену переменных вида

$$
\begin{aligned}
& \dot{\varphi}=\omega_{0}+\mu \Phi(\varphi, \psi, \xi), \\
& J=\frac{a-b}{h-b} \omega_{0}+\mu \mathrm{Y}(\varphi, \psi)+\mu y .
\end{aligned}
$$

Уравнения для функций $\Phi(\varphi, \psi, \xi), \mathrm{Y}(\varphi, \psi)$ и их решения, а также константа второго уравнения находятся по той же схеме, что и в разделах 1.2, 2.1.

В области $D_{\mu}$ замена переменных (2.7) сводит систему (2.6) к стандартной форме: 


$$
\begin{aligned}
& \dot{\xi}=\mu\left(y+\mathrm{Y}-\Phi \frac{\partial \Phi}{\partial \varphi}-\Phi+\Delta\right), \\
& \dot{y}=\mu\left(-h^{*} y-h^{*} Y-\Phi \frac{\partial \mathrm{Y}}{\partial \varphi}+a^{*} \Phi-b^{*} \Delta\right), \\
& \dot{\eta}=\mu \Phi(\xi, \varphi, \eta), \\
& \dot{\varphi}=\omega_{0}+\mu \Phi(\xi, \varphi, \eta),
\end{aligned}
$$

где $\Phi=\frac{1}{\omega_{0}} \cos \varphi-\frac{A}{\omega_{0}} \cos \psi+\xi, \quad Y=\frac{b^{*}}{\omega_{0}} \cos \varphi+\frac{A b^{*}}{\omega_{0}} \cos \psi, \eta=\varphi-\psi-$ фазовая расстройка, $\Delta$ - расстройка частот.

Усредняя систему (2.8) по быстрой фазе, получаем укороченную систему вида

$$
\begin{aligned}
& \dot{\xi}=\mu\left(-\xi+y-\frac{A}{2 \omega_{0}^{2}} \sin \eta+\Delta\right), \\
& \dot{y}=\mu\left(-h^{*} y+a^{*} \xi+b^{*}\left(\frac{A}{2 \omega_{0}^{2}} \sin \eta-\Delta\right)\right), \\
& \dot{\eta}=\mu \xi, \\
& \dot{\varphi}=\omega_{0}+\mu \xi .
\end{aligned}
$$

В системе (2.9) за усредненными переменными сохранены старые обозначения. В свою очередь, преобразованием времени $\mu \frac{2 \omega_{0}^{2}}{A} \tau=\tau_{n}$ и переменной $y=\frac{A}{2 \omega_{0}^{2}} x$ первые три уравнения (2.9) сводятся к следующей эквивалентной системе:

$$
\begin{aligned}
& I_{r} \ddot{\eta}+\dot{\eta}+\sin \eta=\gamma^{r}+x, \\
& \dot{x}+h_{r} x=a_{r} \dot{\eta}+b_{r}\left(\sin \eta-\gamma^{r}\right),
\end{aligned}
$$

где $I_{r}=\frac{A}{2 \omega_{0}^{2}}, \gamma^{r}=\Delta I_{r}^{-1}, h_{r}=h^{*} I_{r}^{-1}, a_{r}=a^{*} I_{r}^{-1}, b_{r}=b^{*} I_{r}^{-1}$. 
Как видим, система (2.10) с точностью до обозначений переменных и параметров совпадает с автономной системой (2.6). Связь с параметрами уравнения третьего порядка вида (2.3) осуществляется по формулам:

$$
\begin{aligned}
& \beta=\sqrt{\frac{A}{2 \omega_{0}^{2}} \frac{\left(h^{*}-b^{*}\right)}{\left(1+h^{*}\right)^{3}}}, \alpha=\sqrt{\frac{A}{2 \omega_{0}^{2}} \frac{1}{\left(h^{*}-b^{*}\right)\left(1+h^{*}\right)}}, \\
& \lambda^{r}=\sqrt{\frac{2 \omega_{0}^{2}}{A}} \frac{h^{*}-a^{*}}{\sqrt{\left(h^{*}-b^{*}\right)\left(1+h^{*}\right)}}, \gamma^{r}=\left(\frac{A}{2 \omega_{0}^{2}}\right)^{-1} \Delta .
\end{aligned}
$$

На рис. 2.7 показаны бифуркационные диаграммы параметров системы (2.10) как аналоги соответствующих диаграмм для автономной системы, изображенных на рис. 2.4, 2.5. На обеих диаграммах область параметров (1) соответствует существованию устойчивого и седлового предельных циклов, причем устойчивый предельный цикл устойчив глобально и соответствует синхронизации вращений ротатора. Эта область называется областью захвата синхронизации: ротатор при любом начальном условии оказывается в режиме синхронизации. Область (2) соответствует существованию устойчивого и седлового предельных циклов, а также устойчивого инвариантного тора $T^{2}$. Для параметров из этой области в зависимости от начальных условий возможен как режим синхронизации, так и режим устойчивых стационарных биений. Область (3) соответствует существованию глобально устойчивого инвариантного тора. Для параметров из этой области режим простой синхронизации невозможен. Заметим, что во всех случаях мы говорим о торах как об инвариантных многообразиях, не упоминая о структурах траекторий на этих торах. На этих многообразиях может быть как квазипериодическая обмотка, так и предельные циклы, соответствующие субгармоническим резонансам. Если при некоторых условиях в системе реализуется тор, то это может соответствовать в одном случае режиму квазипериодических биений, а при малом изменении параметров - режиму субгармонической синхронизации. Однако можно утверждать, что чем меньше параметр $\mu$, тем меньше вероятность существования субгармонического 
резонанса. Для области параметров (4) существуют устойчивый и седловой предельные циклы, а также устойчивый и неустойчивый торы. В отличие от рис. 2.4, 2.5 на рис. 2.7 существуют новые области (6) и (7). Область (6) (толстая кривая Трикоми) соответствует хаотическим аттракторам, связанным с разрушением двумерного инвариантного тора, а также с существованием грубых гомоклинических кривых седлового резонансного предельного цикла. Область (7) (толстая кривая двойного цикла), вероятно, также соответствует хаотическим аттракторам. При переходе параметров из области (4) в область (1) происходит слияние устойчивого и неустойчивого торов. Бифуркация слияния торов не изучена. Вероятно, что перед слиянием торы теряют гладкость. Как правило, это приводит к рождению хаотических аттракторов. Названные области являются «серыми» в том смысле, что их границы не определены, конкретные бифуркации, ведущие к хаотизации динамических процессов, неизвестны. То и другое уточняется в порядке численного эксперимента.

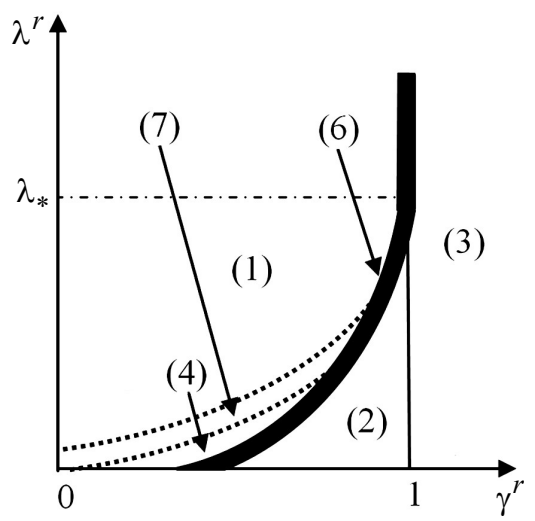

$\mathrm{a}$

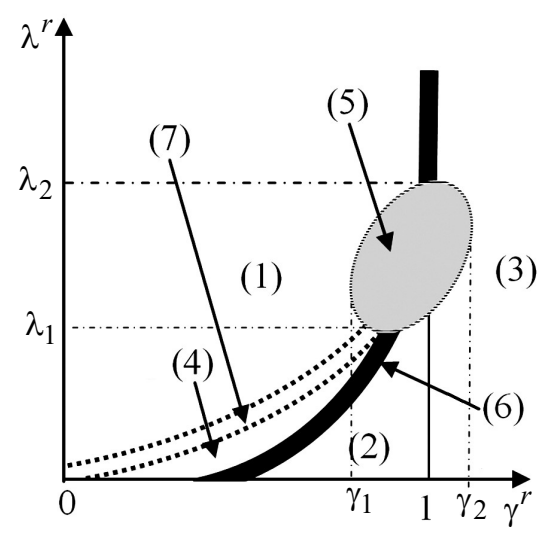

6

Рис. 2.7. Бифуркационные диаграммы параметров системы (2.10) в зоне главного резонанса.

Обсудим вопрос о соответствии хаотических аттракторов исходной системы (2.8) и усредненной системы (2.10). Ясно, что если $A$ - странный аттрактор усредненной системы, то аттрактор исходной системы не является формальным произведением $A \times S^{1}$ в силу негрубости аттрактора $A$ : гомоклинические траектории не имеют 
однозначной интерпретации. С другой стороны, при уменьшении параметра $\mu$ области параметров (6) и (7) на рис. 2.7 стягиваются к линиям, аналогичным бифуркационным кривым на рис. 2.4, 2.5, а область (5) сжимается к соответствующей ей области на рис. 2.5. Следовательно, сближаются и соответствующие бифуркационные кривые в этих областях, в частности кривые удвоения периода цикла в системе (2.4) и кривые удвоений одного из периодов тора в системе (2.6). Другими словами, можно утверждать, что для любого $\mu=\mu_{0}$ хаотический аттрактор исходной системы содержит $N\left(\mu_{0}\right)$ торов седлового типа («костяк» хаотического аттрактора), каждый из которых соответствует предельному циклу усредненной системы, причем $\lim _{\mu_{0} \rightarrow 0} N\left(\mu_{0}\right)=\infty$. По этой причине в дальнейшем будем говорить, что хаотический аттрактор имеет тот же самый тип, что и аттрактор усредненной системы, помня, однако, что это соответствие не совсем точно. В указанном смысле бифуркации торов в системе (2.6) должны происходить по тому же сценарию, что и бифуркации предельных циклов в усредненной системе (2.10). Картины фазовых траекторий системы (2.1) и картины траекторий точечных отображений для системы (2.6) (на соответствующих плоскостях) должны содержать одинаковые качественные особенности.

Замечание. Уравнения (2.10) инвариантны к преобразованию $\left(\eta, x, \gamma^{r}\right) \rightarrow\left(-\eta,-x,-\gamma^{r}\right)$. Это значит, что к бифуркационным диаграммам, изображенным на рис. 2.7, следует добавить их отражения относительно оси $\lambda^{r}$. В результате получится полная картина бифуркаций динамических режимов при приближении к резонансной ступени как слева, так и справа. Считаем, что это проделано. В таком случае, если параметр $\gamma^{r}$ движется из области (3) в область (1) (см. рис. 2.7), то это соответствует движению к резонансу справа, а если он движется так же, но в левой части диаграммы, то это соответствует движению к резонансу слева.

Численный эксперимент. При проведении численного эксперимента нас будут интересовать бифуркации динамических режимов ротатора при переходе от режима биений к режиму синхронизации, т. е. при переходе параметра $\gamma$ (при постоянстве всех других параметров) из области (3) в область (1) (см. рис. 2.7). 
При переходе параметра $\gamma$ через область (6) характер бифуркаций тот же, что и для неавтономного ротатора (см. раздел 1.2), поэтому данный случай рассматривать не будем. Остановимся на случае перехода из (3) в (1) через область (5) как наиболее интересном для иллюстрации наших теоретических результатов. Наряду с исследованием фазовых портретов в эксперименте исследовались структуры траекторий точечного отображения $(\varphi, x, J)_{\psi=\psi_{0}} \rightarrow(\bar{\varphi}, \bar{x}, \bar{J})_{\psi=\psi_{0}+2 \pi}$, строившегося для эквивалентной системы (2.6) вида

$$
\begin{aligned}
& \dot{\varphi}=\gamma+x, \\
& \dot{x}=\mu(-x-\sin \varphi+J+A \sin \psi), \\
& \dot{J}=-h J+a(\gamma+x)+b(\sin \varphi-\gamma-A \sin \psi), \\
& \dot{\psi}=\omega_{0} .
\end{aligned}
$$

Эксперимент 1. Значения параметров: $\mu=h=0,006$, $a=-0,006, \quad b=0,003, \quad A=5, \omega_{0}=0,5$. При этих параметрах $\lambda^{r}=0,632$. Галерея фазовых портретов и соответствующих им точечных отображений представлена на рис. 2.8. Движение параметра $\gamma$ к резонансной ступени происходит справа.

При $\gamma=2,0892$ ротатор находится в режиме квазипериодических биений (рис. 2.8,a). Левый рисунок ряда - тор с квазипериодической обмоткой на развертке фазового цилиндра, средний и правый рисунки - траектория точечного отображения этого тора. Дискретность траектории слабо выражена из-за большой плотности точек. При уменьшении $\gamma$ происходит бифуркация удвоения периода тора (рис. 2.8,б). Далее тор, не испытывая дальнейших удвоений, изменяет конфигурацию (рис. 2.8,в). Затем следует обратная бифуркация удвоения (рис. $2.8, \Gamma)$. Получившийся тор теряет устойчивость, и ротатор скачком входит в режим синхронизации (рис. 2.8,д). На этом рисунке изображена траектория предельного цикла на развертке цилиндра.

При $\gamma=1,9412$ ротатор скачком из режима синхронизации переходит в режим квазипериодических биений (срыв с нижнего конца резонансной ступени, рис. 2.8,e) - тор удвоенного периода (сравните с рис. 2.8,в). Если при этом параметр $\gamma$ увеличивать, то 
этот тор испытает обратную бифуркацию удвоения аналогично переходу от рис. 2.8 ,в к рис. 2.8 ,г. Далее последует потеря устойчивости этого тора и совершится скачок слева на резонансную ступень. Некоторое визуальное различие случаев на рис. 2.8 , е и 2.8 ,в связано с выбором секущей плоскости. При переходе от рис. 2.8,e к рис. 2.8,3 тор изменяет конфигурацию, а затем испытывает обратную бифуркацию удвоения (рис. 2.8,и).

Таким образом, в данном случае динамика ротатора при переходе в режим синхронизации является регулярной. Обратим внимание на одинаковые бифуркационные сценарии перехода в режим синхронизации как слева, так и справа от резонансной ступени. Это было декларировано выше как следствие инвариантности усредненной системы к соответствующим преобразованиям.
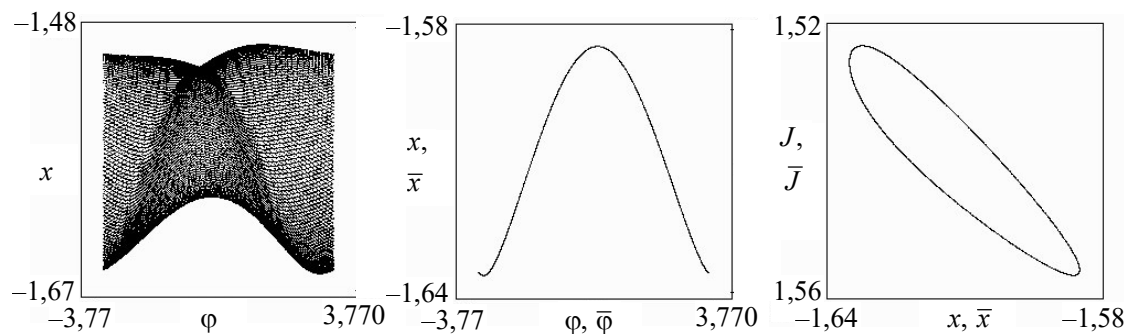

$\gamma=2,0892$

a
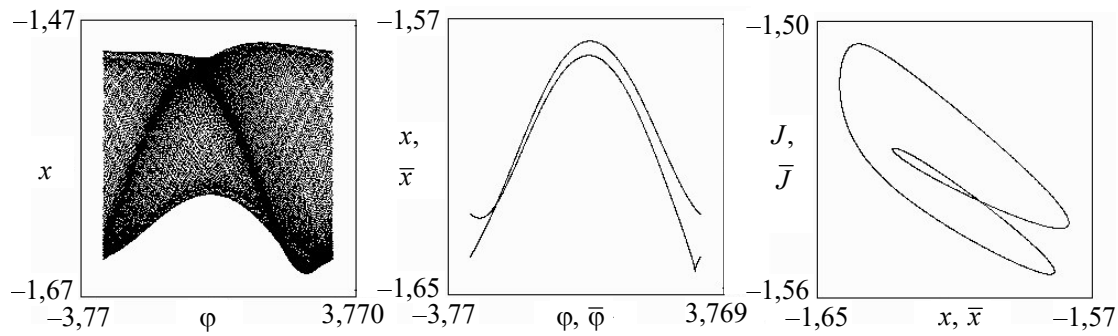

$$
\gamma=2,0842
$$

6

Рис. 2.8. Галерея фазовых портретов и портретов точечного отображения в эксперименте 1 (см. также с. 60-62). 

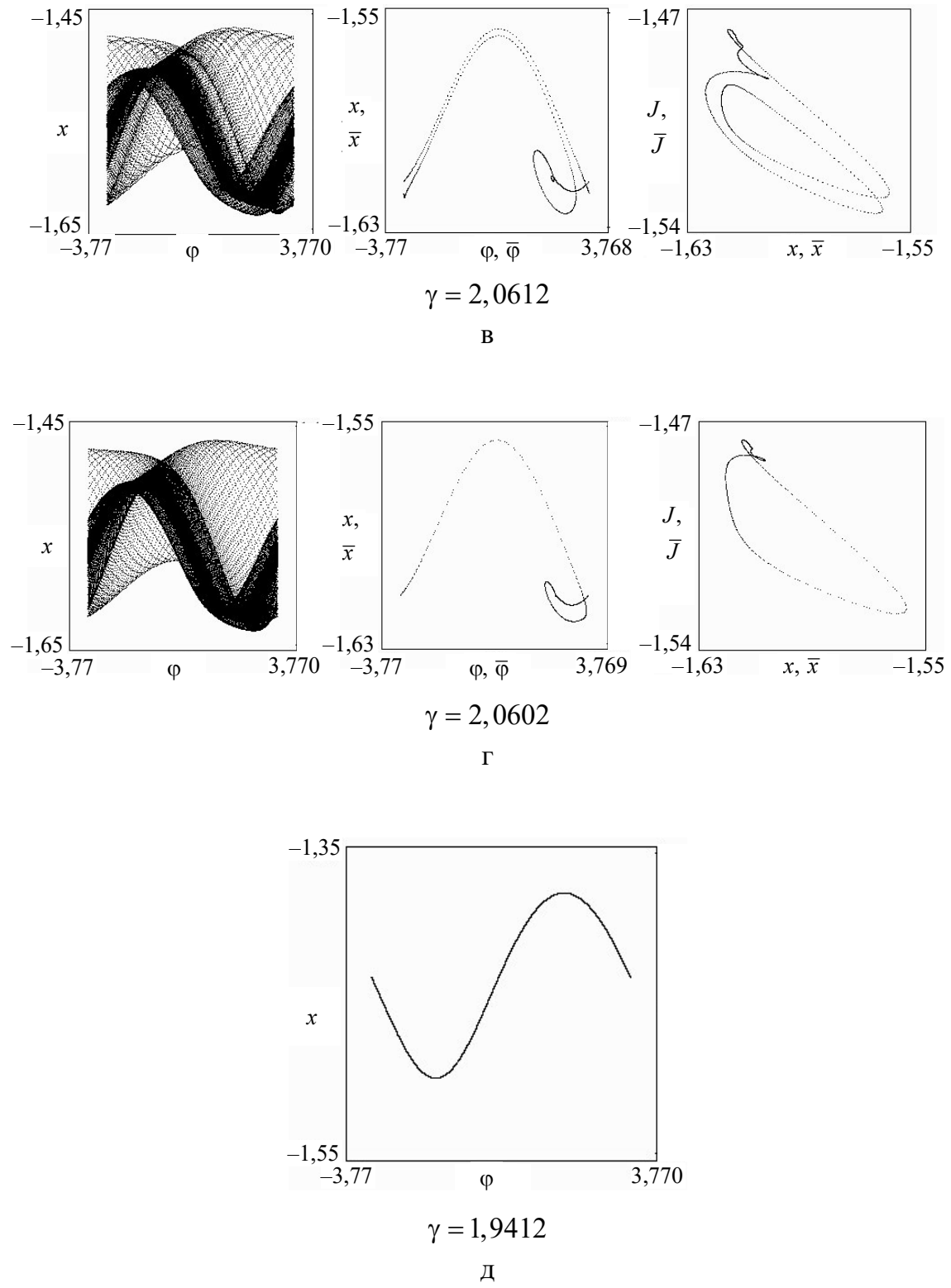

Рис. 2.8. Продолжение. 

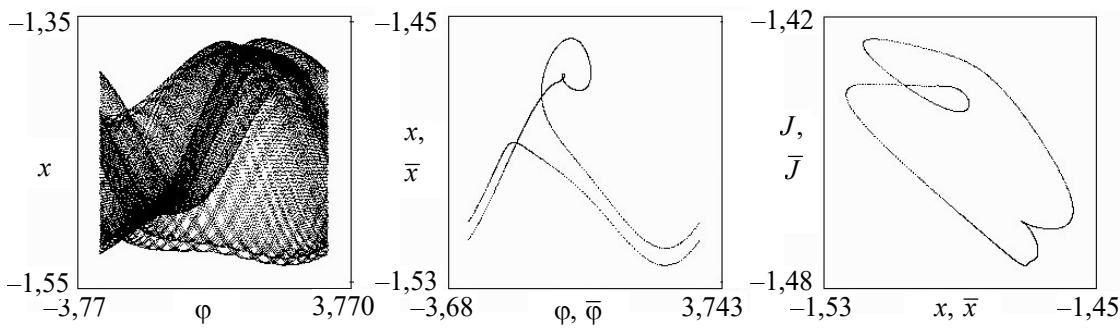

$$
\begin{gathered}
\gamma=1,9362 \\
\text { e }
\end{gathered}
$$
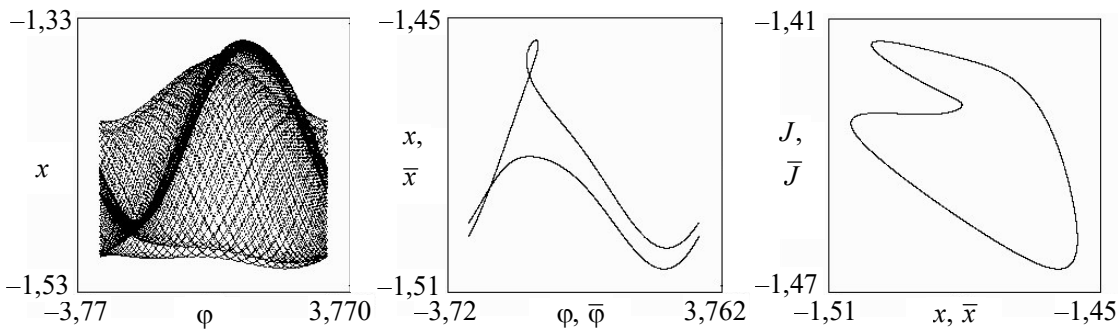

$$
\begin{gathered}
\gamma=1,9182 \\
\text { ж }
\end{gathered}
$$
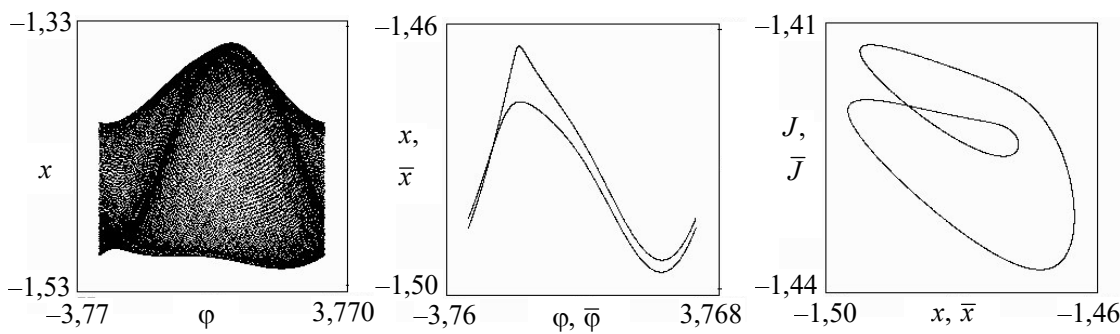

$$
\gamma=1,9152
$$

3

Рис. 2.8. Продолжение. 

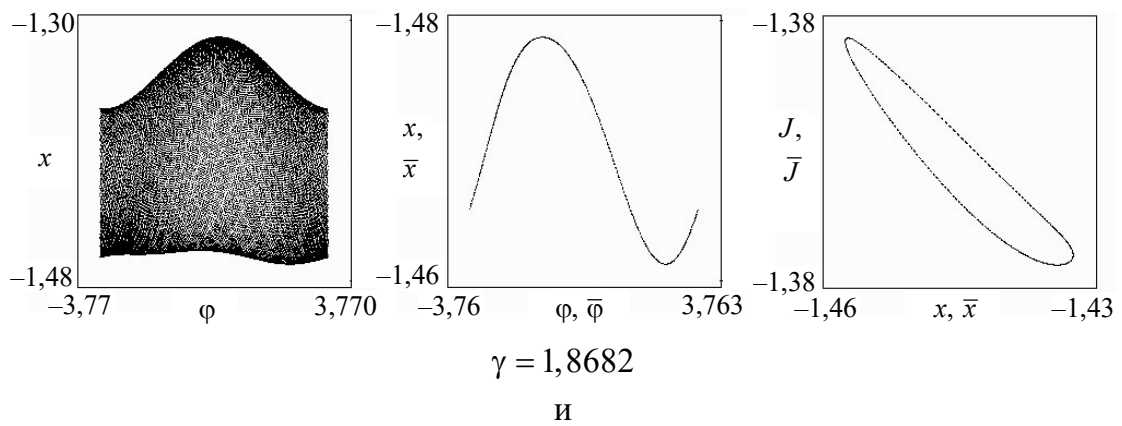

Рис. 2.8. Окончание.

Эксперимент 2. Значения параметров: $\mu=0,086, \quad h=0,006$, $a=-0,006, b=0,003, A=2,5, \omega_{0}=0,8$. В этом случае $\lambda^{r}=0,517$. Галерея фазовых портретов и соответствующих портретов точечных отображений представлена на рис. 2.9. Движение параметра $\gamma$ к резонансной ступени справа.

При $\gamma=3,6091$ имеется квазипериодическое движение ротатора (рис. 2.9,a). С уменьшением параметра тор испытывает две бифуркации удвоения периода, оставаясь при этом эргодическим и «гладким» (рис. 2.9,б,в) (сравните с рис. 2.6,б,в). После следующей бифуркации удвоения тор теряет гладкость (рис. 2.9,г). Цепочка удвоений приводит к рождению странного аттрактора Фейгенбаума (рис. 2.9,д) (сравните с рис. 2.6,ж). Далее к этому аттрактору, подобно автономному случаю, «подмешивается» аттрактор Шильникова. При уменьшении параметра на объединенном аттракторе рождается устойчивый тор с периодом, равным четырем (рис. 2.9,e). Далее тор испытывает обратную бифуркацию удвоений, оставаясь при этом «гладким» (рис. 2.9,ж-и). Наконец, тор одинарного периода теряет устойчивость, и ротатор скачком переходит в режим синхронизации (рис. 2.9,к, где изображена фазовая траектория). 

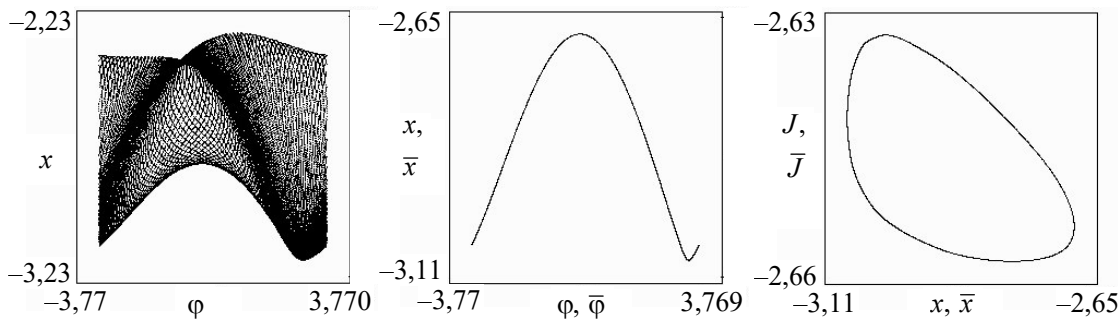

$$
\gamma=3,6091
$$

$\mathrm{a}$
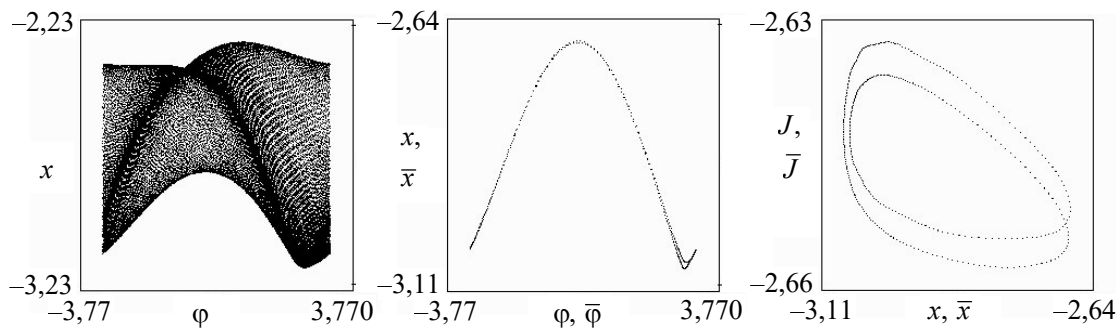

$$
\gamma=3,6051
$$

6
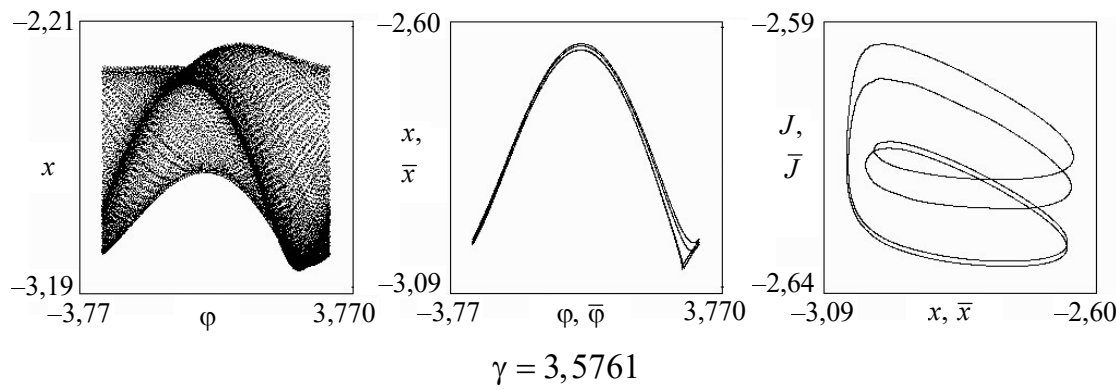

B

Рис. 2.9. Галерея фазовых портретов и портретов точечного отображения в эксперименте 2 (см. также с. 64-66). 

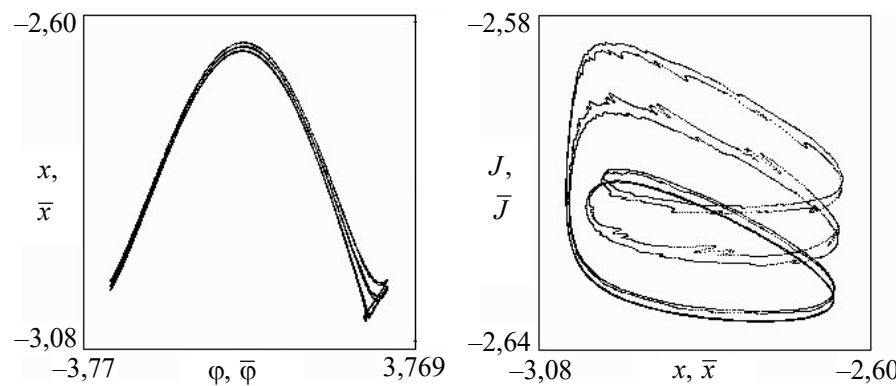

$$
\gamma=3,5741
$$

$\Gamma$
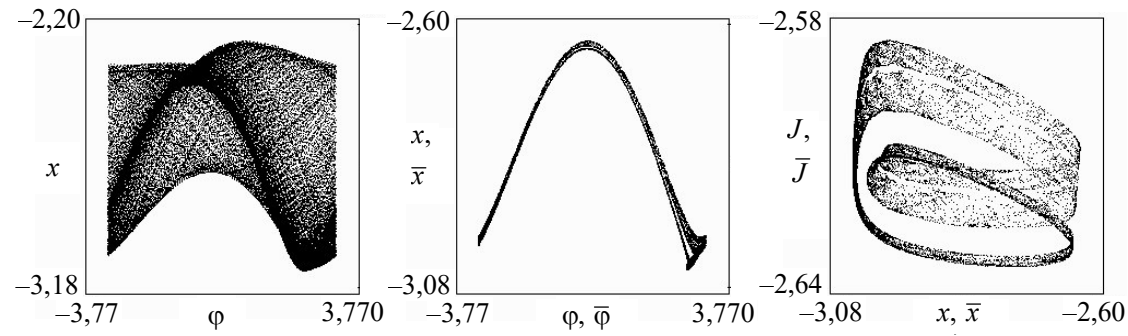

$$
\gamma=3,5731
$$

Д
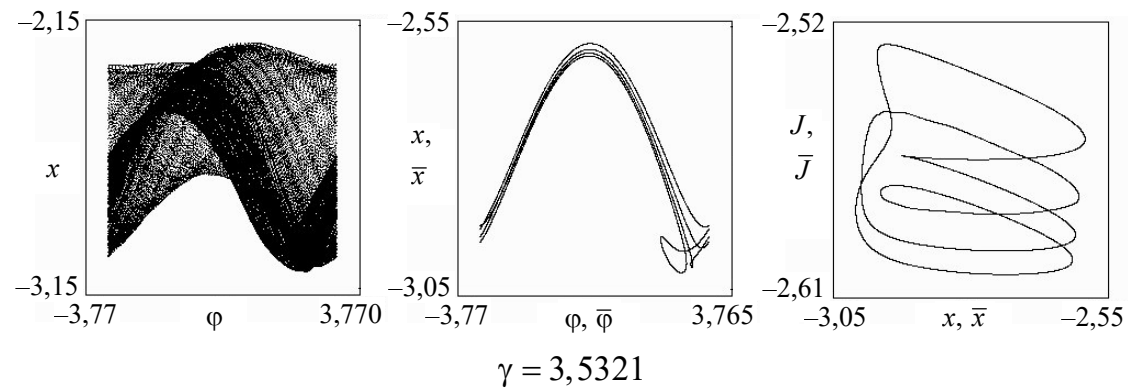

$\mathrm{e}$

Рис. 2.9. Продолжение. 

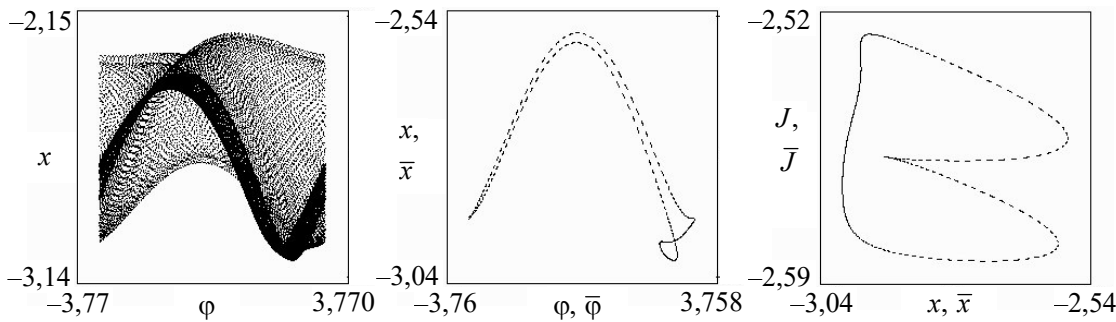

$$
\gamma=3,5251
$$

Ж
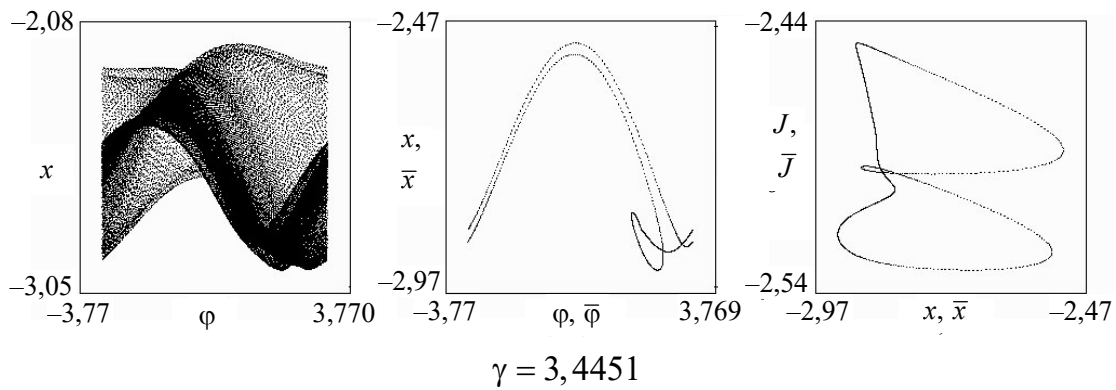

3
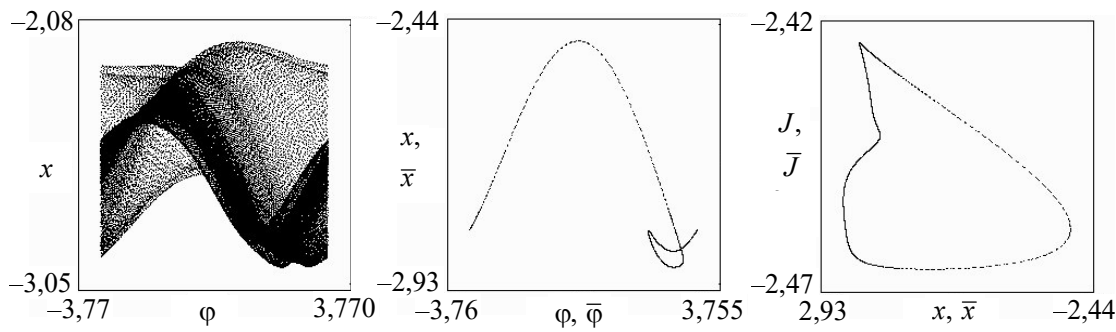

$$
\gamma=3,4091
$$

И

Рис. 2.9. Продолжение. 


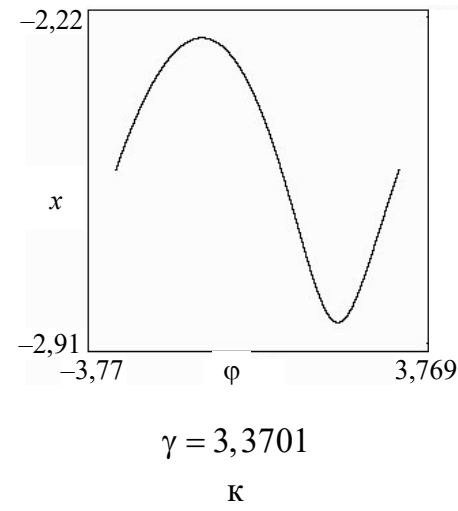

Рис. 2.9. Окончание.

Эксперимент 3. В предыдущем эксперименте тор по мере удвоений потерял гладкость. Возникает вопрос: является это правилом или нет? Выясним это. Значения параметров: $\mu=0,006$, $h=0,004, a=-0,006, \quad b=0, A=5, \omega_{0}=0,5$, при этом $\lambda^{r}=0,5$. Движемся к резонансу слева. При $\gamma=1,19534$ (рис. 2.10,a) имеется гладкий удвоенный тор. При увеличении параметра тор, не теряя гладкости, испытывает каскад удвоений (рис. 2.10,б,в). В результате получаем аттрактор Фейгенбаума (рис. 2.10,г) на базе удвоений гладкого тора (сравните с рис. 2.6,ж).
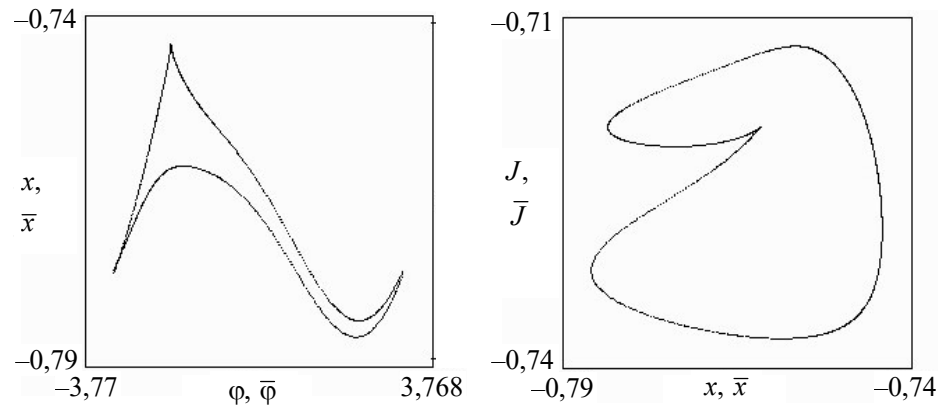

$$
\gamma=1,19534
$$

a

Рис. 2.10. Галерея фазовых портретов и портретов точечного отображения в эксперименте 3 (см. также с. 67). 


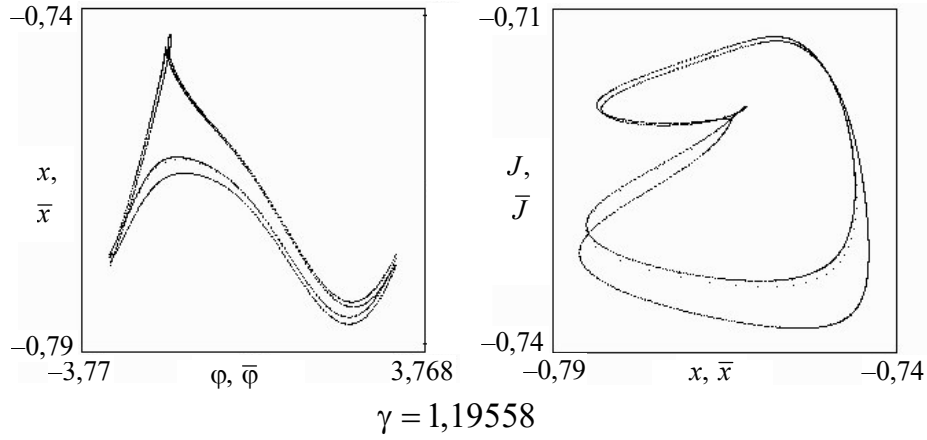

6
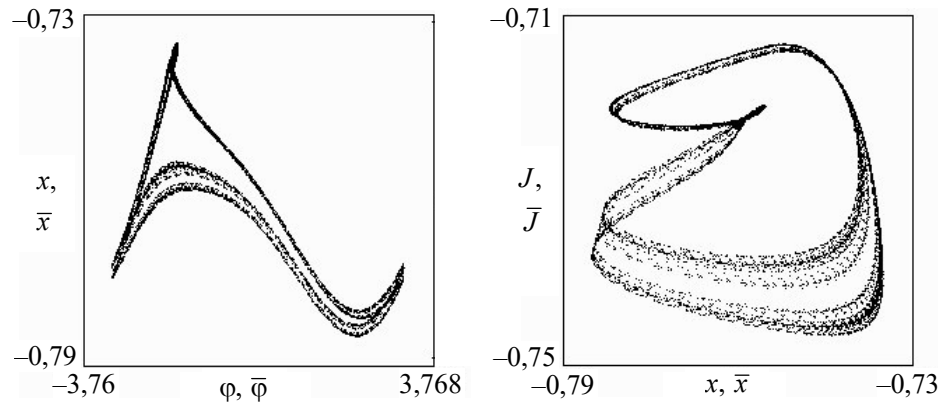

$$
\gamma=1,19563
$$

B
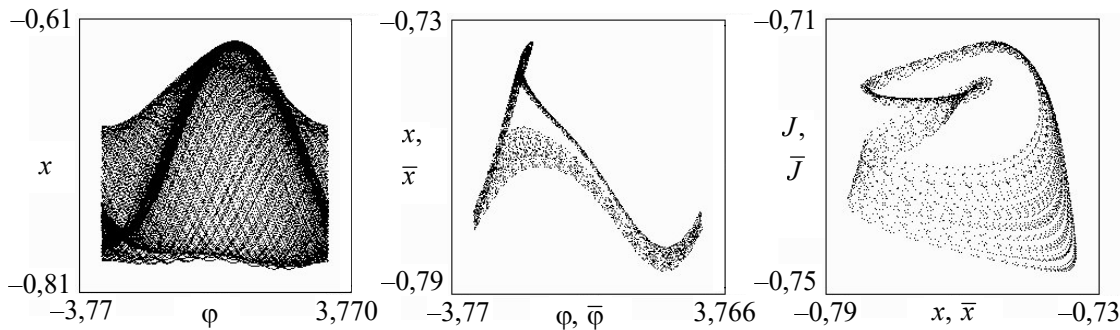

$$
\gamma=1,19567
$$

$\Gamma$

Рис. 2.10. Окончание.

В заключение обратим внимание на механизм возврата ротатора на нулевую резонансную ступень - из режима вращений в режим колебательных движений. На рис. 2.11,а показана траектория 
сложного вращательно-колебательного движения ротатора и соответствующая дискретная траектория точечного отображения. Эта траектория является предельным циклом (!) чрезвычайно большого периода. Темная часть соответствует сгущению траектории. При уменьшении $\gamma$ из сгущения траектории рождается кратный колебательный цикл. В момент бифуркации период вращательноколебательного предельного цикла становится бесконечным. Такая бифуркация называется «катастрофа голубого неба» [24, 31]. При дальнейшем уменьшении параметра кратный цикл распадается на два - устойчивый и неустойчивый, и далее ротатор движется на устойчивом колебательном цикле (рис. 2.11,б).

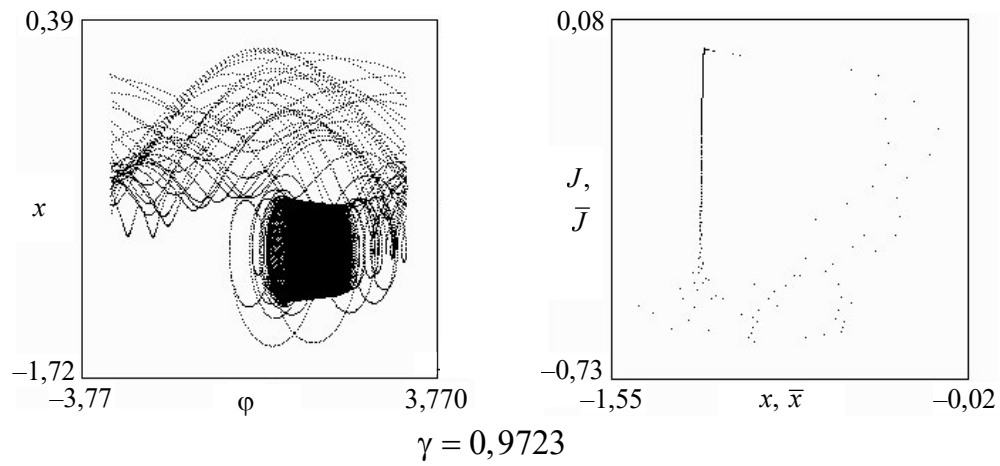

a

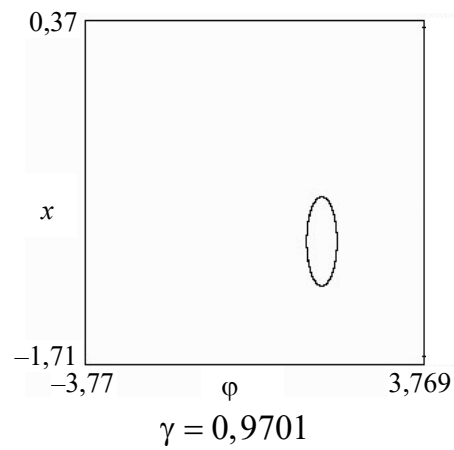

6

Рис. 2.11. Бифуркация «катастрофа голубого неба» и колебательный режим ротатора: $\mu=0,086, h=0,006, a=-0,006, b=0,003$,

$$
A=2,5, \omega_{0}=0,8 \text {. }
$$


Глава 3

\section{АВТОНОМНЫЕ СИСТЕМЫ С ДВУМЯ СТЕПЕНЯМИ СВОБОДЫ}

\section{1. Динамика ротатора с колебательной нагрузкой}

Рассмотрим систему, схема которой изображена на рис. 3.1. Здесь ротатором является асинхронный электродвигатель с жестким валом и жестко посаженным несбалансированным диском, а осциллятором - муфта, упруго связанная с диском и осциллирующая относительно оси вала. Она и представляет колебательную нагрузку. Предполагаем, что вал расположен горизонтально, муфта не смещается вдоль вала, а трение между муфтой и валом является вязким.

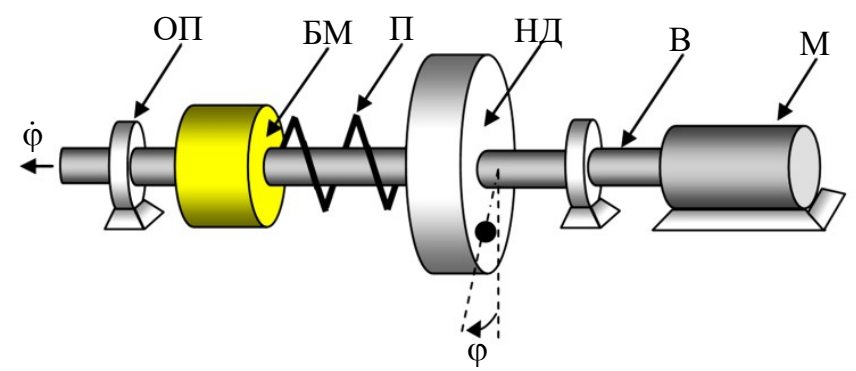

Рис. 3.1. Асинхронный мотор с несбалансированным диском, упруго связанным с инерционной муфтой: $\mathrm{M}$ - асинхронный мотор,

В - жесткий вал, НД - несбалансированный диск, П - пружина, БМ - муфта, ОП - опорный подшипник.

Построим динамическую модель системы, используя ее функцию Лагранжа:

$$
L=T+V=\frac{I_{1}}{2} \dot{\varphi}^{2}+\frac{I_{0}}{2} \dot{\vartheta}^{2}+m g e(1-\cos \varphi)+\frac{k}{2}(\varphi-\vartheta)^{2},
$$

где $\varphi, \vartheta$ - углы поворота диска и муфты; $I_{1}, I_{0}$ - моменты инерции диска и муфты; $k$ - крутильная жесткость пружины. 
Предполагаем, что движущий момент двигателя является линейной функцией скорости вращения $M_{d}=M_{0}-\lambda \dot{\varphi}$, а момент силы трения, действующей на муфту со стороны вала, $\delta_{0}(\dot{\varphi}-\dot{\theta})$. В таком случае уравнения движения системы имеют следующий вид:

$$
\begin{aligned}
& I_{1} \ddot{\varphi}+m g e \sin \varphi+k(\varphi-\vartheta)=M_{0}-\lambda \dot{\varphi}, \\
& I_{0} \ddot{\vartheta}+k(\vartheta-\varphi)=\delta_{0}(\dot{\varphi}-\dot{\vartheta}) .
\end{aligned}
$$

После введения новой переменной $\frac{k}{m g e}(\vartheta-\varphi)=J$ и времени $\frac{m g e}{\lambda} \tau=\tau_{n}$ модель принимает окончательный вид:

$$
\begin{aligned}
& I \ddot{\varphi}+\dot{\varphi}+\sin \varphi=\gamma+J, \\
& \ddot{J}+\delta \dot{J}+\omega_{0}^{2} J=b \ddot{\varphi},
\end{aligned}
$$

где $\quad I=\frac{I_{1} m g e}{\lambda^{2}}, \quad \gamma=\frac{M_{0}}{m g e}, \quad \delta=\delta_{0} I_{0}^{-1} \frac{\lambda}{m g e}, \quad \omega_{0}^{2}=k I_{0}^{-1}\left(\frac{\lambda}{m g e}\right)^{2}$, $b=-\frac{k}{m g e}$. Обратим внимание на то, что переменная $J$ имеет смысл угла закрутки муфты относительно вала.

Система (3.1) является случаем системы ротатор - осииллятор [32]. Проведем ее исследование для области параметров $D_{\mu}=\left\{I^{-1}=\mu \square 1, \delta=\mu h, \gamma-\omega_{0}=\mu \Delta\right\}$, физически соответствующей квазилинейному ротатору, резонансно взаимодействующему с высокодобротным осциллятором. Преобразование к стандартной форме проведем для эквивалентной системы вида

$$
\begin{aligned}
& I \ddot{\varphi}+\dot{\varphi}+\sin \varphi=\gamma+J, \\
& \dot{J}=W+b \dot{\varphi}-\mu h J, \\
& \dot{W}=-\omega_{0}^{2} J .
\end{aligned}
$$

В уравнениях осциллятора выполним модифицированную замену Ван дер Поля, а уравнение ротатора преобразуем уже известным способом: 


$$
\begin{aligned}
& J=\theta \sin \varphi+\eta \cos \varphi, \\
& W=(\theta \cos \varphi-\eta \sin \varphi) \omega_{0}-b \omega_{0}, \\
& \dot{\varphi}=\omega_{0}+\mu \Phi(\theta, \eta, \varphi, \xi) .
\end{aligned}
$$

Эта замена приводит уравнения осциллятора к эквивалентной системе:

$$
\begin{aligned}
& \dot{\theta}=\mu \Theta(\theta, \eta, \xi, \varphi), \\
& \dot{\eta}=\mu T(\theta, \eta, \xi, \varphi),
\end{aligned}
$$

где $\Theta=(\eta+b \sin \varphi) \Phi-h(\theta \sin \varphi+\eta \cos \varphi) \sin \varphi, \quad T=(-\theta+b \cos \varphi) \Phi-$ $-h J \cos \varphi$.

Подстановка последнего уравнения (3.3) в первое уравнение (3.2) дает уравнение

$$
\frac{\partial \Phi}{\partial \theta} \mu \Theta+\frac{\partial \Phi}{\partial \eta} \mu T+\frac{\partial \Phi}{\partial \varphi} \omega_{0}+\frac{\partial \Phi}{\partial \varphi} \mu \Phi+\mu \Phi+\dot{\xi}=\mu \Delta+(\theta-1) \sin \varphi+\eta \cos \varphi,
$$

из которого, в свою очередь, следуют уравнения, определяющие функции $\Phi(\varphi, \theta, \eta, \xi), \Xi(\varphi, \theta, \eta, \xi)$, а следом и сами эти функции:

$$
\begin{gathered}
\frac{\partial \Phi}{\partial \varphi} \omega_{0}=(\theta-1) \sin \varphi+\eta \cos \varphi, \Phi=\frac{1-\theta}{\omega_{0}} \cos \varphi+\frac{\eta}{\omega_{0}} \sin \varphi+\xi, \\
\Xi=\Delta-\left(\frac{\partial \Phi}{\partial \theta} \Theta+\frac{\partial \Phi}{\partial \eta} \mathrm{T}+\frac{\partial \Phi}{\partial \varphi} \Phi+\Phi\right) .
\end{gathered}
$$

Конечный результат преобразований - эквивалентная уравнениям (3.1) система в стандартной форме:

$$
\begin{aligned}
& \dot{\theta}=\mu \Theta(\theta, \eta, \xi, \varphi), \\
& \dot{\eta}=\mu T(\theta, \eta, \xi, \varphi), \\
& \dot{\xi}=\mu \Xi(\theta, \eta, \xi, \varphi), \\
& \dot{\varphi}=\omega_{0}+\mu \Phi(\theta, \eta, \xi, \varphi) .
\end{aligned}
$$

Усредняя систему (3.4) по быстрой фазе, получаем укороченную систему вида 


$$
\begin{aligned}
& \dot{\xi}=\mu\left(-\xi+b_{3} \eta+\Delta\right), \\
& \dot{\theta}=\mu\left(-b_{4} \theta+b_{5} \eta+\eta \xi\right), \\
& \dot{\eta}=\mu\left(-b_{4} \eta-b_{5} \theta-\theta \xi+b_{7}\right), \\
& \dot{\varphi}=\omega_{0}+\mu \xi,
\end{aligned}
$$

где $b_{3}=\frac{1}{2 \omega_{0}^{2}}, b_{4}=\frac{h}{2}, b_{5}=\frac{b}{2 \omega_{0}}, b_{7}=\frac{b}{2 \omega_{0}}$.

В свою очередь, заменой переменных и времени

$$
x=b_{4}^{-1}\left(\xi+b_{5}\right), y=\frac{b_{3}}{b_{4}} \eta-\Lambda, z=\frac{b_{3}}{b_{4}} \theta, \mu b_{4} \tau=\tau_{n}
$$

первые три уравнения (3.5) сводятся к системе вида

$$
\begin{aligned}
& \dot{x}=-\sigma(x-y)+\rho, \\
& \dot{y}=-y-x z, \\
& \dot{z}=-z+x y+\Lambda x,
\end{aligned}
$$

где $\sigma=\frac{1}{b_{4}}, \rho=\frac{\left(\Delta+\left(b_{4} b_{5}+b_{3} b_{7}\right) / b_{4}\right)}{b_{4}^{2}}, \Lambda=\frac{b_{3} b_{7}}{b_{4}^{2}}$.

Перечислим некоторые свойства усредненной системы (3.6).

1. Система является диссипативной. Свойство устанавливается при помощи квадратичной формы $V=\frac{1}{2}\left(x^{2}+(y+\Lambda)^{2}+(z-\sigma)^{2}\right)$, производная которой, вычисленная вдоль траекторий системы (3.6), имеет вид $\dot{V}=-\sigma x^{2}-(\rho-\sigma \Lambda) x-y^{2}-\Lambda y-z^{2}+\sigma z$. Очевидно, она является отрицательной вне некоторого шара $V \leq L^{2}$. Это значит, что состояния равновесия, предельные циклы и все другие предельные траектории (если таковые существуют) находятся в ограниченной области фазового пространства системы - в шаре.

2. В зависимости от параметров система имеет от одного до трех состояний равновесия, координаты которых

$$
x_{0}=\omega, y_{0}=-\frac{\Lambda \omega^{2}}{1+\omega^{2}}, z_{0}=\frac{\Lambda \omega}{1+\omega^{2}},
$$

a $\omega_{1,2,3}$ являются решениями уравнения 


$$
f=\omega+\frac{\Lambda \omega^{2}}{1+\omega^{2}}=\frac{\rho}{\sigma} .
$$

Заметим, что введенный выше параметр $\omega$ пропорционален расстройке частот ротатора и осциллятора. Далее кривую $f=f(\omega)$ будем называть кривой состояний равновесия.

3. Если система имеет одно состояние равновесия $O\left(x_{0}, y_{0}, z_{0}\right)$, то оно глобально асимптотически устойчиво (ГАУ). Свойство доказывается при помощи функции Ляпунова $V=\frac{1}{2}\left(m x_{1}^{2}+y_{1}^{2}+z_{1}^{2}\right)$, где $x_{1}=x-x_{0}, y_{1}=y-y_{0}, z_{1}=z-z_{0}$, производная которой по направлению векторного поля системы имеет вид $\dot{V}=-\left(\alpha_{1} x_{1}+y_{1}\right)^{2}-\left(\alpha_{2} x_{1}+z_{1}\right)^{2} \leq 0, \forall\left(x_{1}, y_{1}, z_{1}\right), \quad$ где $\quad \alpha_{1}=\frac{\sigma m-z_{0}}{2}$, $\alpha_{2}=\frac{-\left(y_{0}+\Lambda\right)}{2}$, а $m$ - положительный корень уравнения $\sigma^{2} m^{2}-$ $-2 \sigma\left(z_{0}+2\right) m+z_{0}^{2}+\left(y_{0}+\Lambda\right)^{2}=0$. Можно убедиться, что условия существования положительного корня этого уравнения и условия единственности состояния равновесия в системе (3.6) (условия взаимной однозначности функции $f$ ) совпадают.

Нетрудно выяснить, что условием взаимной однозначности функции $f$ является неравенство $|\Lambda| \leq \frac{8 \sqrt{3}}{9}$.

4. Устойчивость состояний равновесия. Характеристическое уравнение для произвольного состояния равновесия $O\left(x_{0}, y_{0}, z_{0}\right)$ системы имеет вид

$$
p^{3}+a_{0} p^{2}+a_{1} p+a_{2}=0
$$

где $\quad a_{0}=\sigma+2, \quad a_{1}=2 \sigma+1+\omega^{2}+\Lambda \sigma \frac{\omega}{1+\omega^{2}}, \quad a_{2}=\sigma\left(1+\omega^{2}\right) \times$ $\times\left(1+2 \Lambda \frac{\omega}{\left(1+\omega^{2}\right)^{2}}\right)$. Если $\omega=0 \quad(\rho=0)$, то состояние равновесия 
$O(0,0,0)$ является устойчивым. Если $\omega \neq 0$, то условия Гурвица эквивалентны неравенствам

$$
\begin{gathered}
a_{1}>0: f>(<) f_{1}, \\
a_{2}>0: f>(<) f_{2}, \\
a_{0} a_{1}-a_{2}>0: f>(<) f_{3} \text { при } \omega>0(\omega<0), \\
\text { где } f_{1}=-\omega \frac{\sigma+1+\omega^{2}}{\sigma}, \quad f_{2}=\frac{\omega\left(1-\omega^{2}\right)}{2}, \quad f_{3}=-\omega \frac{\sigma^{2}+4 \sigma+2+2 \omega^{2}}{\sigma^{2}} .
\end{gathered}
$$

Другими словами, условием устойчивости любого состояния равновесия системы является расположение соответствующей ему точки кривой состояний равновесий (3.7) выше (ниже) всех кривых $f_{1,2,3}$ при $\omega>0(\omega<0)$. В пространстве параметров $(\sigma, \Lambda, \rho)$ уравнения $f=f_{1,2,3}, f=\rho / \sigma$ определяют бифуркационные поверхности, на которых происходят изменения характера устойчивости состояний равновесия.

Нетрудно установить два простых свойства вспомогательных функций:

1) $f_{2}$ пересекает $f$ в начале координат и в точках экстремума;

2) кроме нуля, все кривые пересекаются между собой в двух точках $\omega_{1,2}= \pm \sqrt{\frac{3 \sigma+2}{\sigma-2}}$.

Приведенную выше информацию о свойствах системы будем использовать при рассмотрении характеристики вращения ротатора.

Характеристика вращения ротатора. Как всегда, наш интерес связан с качественными картинами XВ, а значит, с исследованием различных типов предельных движений ротатора. Точнее, интерес представляет поведение его скорости вращения в зависимости от постоянной составляющей движущего момента $(\gamma)$ при различных значениях других параметров.

По определению ХВ, а также в силу принципа усреднения и вида последнего уравнения (3.5) получаем $\langle\dot{\varphi}\rangle_{\tau}=\omega_{0}+\mu\langle\xi(\tau)\rangle_{\tau}$. Это уравнение определяет ХВ в резонансной зоне колебательной системы - в окрестности частоты $\omega_{0}$, поскольку $\gamma \sim \Delta \sim \rho$, 
a $\xi(t) \sim x(t)$ (в силу замен переменных и параметров). Получаем, что ХВ и кривая $\rho=\rho\left(\overline{x^{*}\left(t, t_{0}\right)}\right)$ имеют одинаковые качественные особенности: одна получается из другой простым пересчетом. Последнюю кривую и будем рассматривать в качестве ХВ. Здесь $x^{*}\left(t, t_{0}\right)$ - решения усредненной системы (3.6), соответствующие ее предельным траекториям: состояниям равновесия, предельным циклам, торам и странным аттракторам.

Рассмотрим подробно кривую состояний равновесия, которая является частью ХВ. Она взаимно однозначна и устойчива при $|\Lambda| \leq \frac{8 \sqrt{3}}{9}$ (рис. $\left.3.2, a\right)$. При $|\Lambda|>\frac{8 \sqrt{3}}{9}$ кривая проходит через начало координат и имеет два экстремума в правой полуплоскости (рис. $3.2,6$, в). Нетрудно убедиться, что если $\frac{d f}{d \omega}<0$, то и $a_{2}<0$, т. е. все точки падающего участка кривой (между экстремумами) неустойчивы и соответствуют состояниям равновесия типа седло или седло-фокус.

Устойчивость/неустойчивость других участков кривой состояний равновесия устанавливается по поведению вспомогательных функций. Как видим, кривая $f_{2}$ проходит через точки экстремума и определяет неустойчивость падающего участка. Для точек возрастающих участков неравенства (см. условия устойчивости) относительно функции $f$ и $f_{2}$ выполняются. Все сводится к взаимному расположению функций $f$ и $f_{1}$, а также $f$ и $f_{3}$. Для точек возрастающего участка в левой полуплоскости $(\omega<0) f_{1}>0, f_{3}>0$, a $f<0$, т. е. условия устойчивости выполняются. Для точек возрастающего участка в правой полуплоскости - наоборот: $f_{1}<0$, $f_{3}<0$, а $f>0$ и соответствующие неравенства также выполняются. Таким образом, все точки левого возрастающего участка кривой $f=f(\omega)$ соответствуют устойчивым состояниям равновесия. 

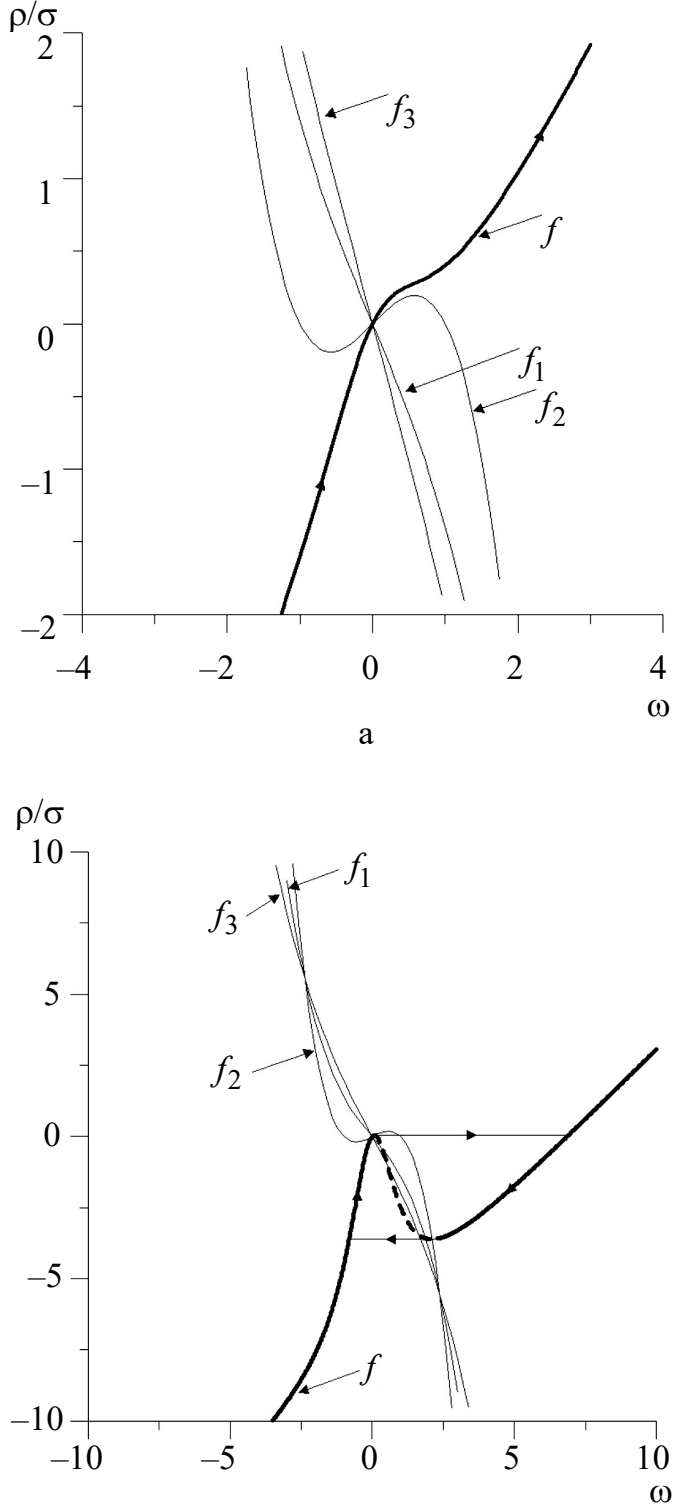

б

Рис. 3.2. Качественные картины ХВ ротатора: $\mathrm{a}-\sigma=5, \Lambda=-1,2$; $\sigma-\sigma=5, \Lambda=-7 ;$ в $-\sigma=5, \Lambda=-25$ (окончание рис. см. на с. 77) 


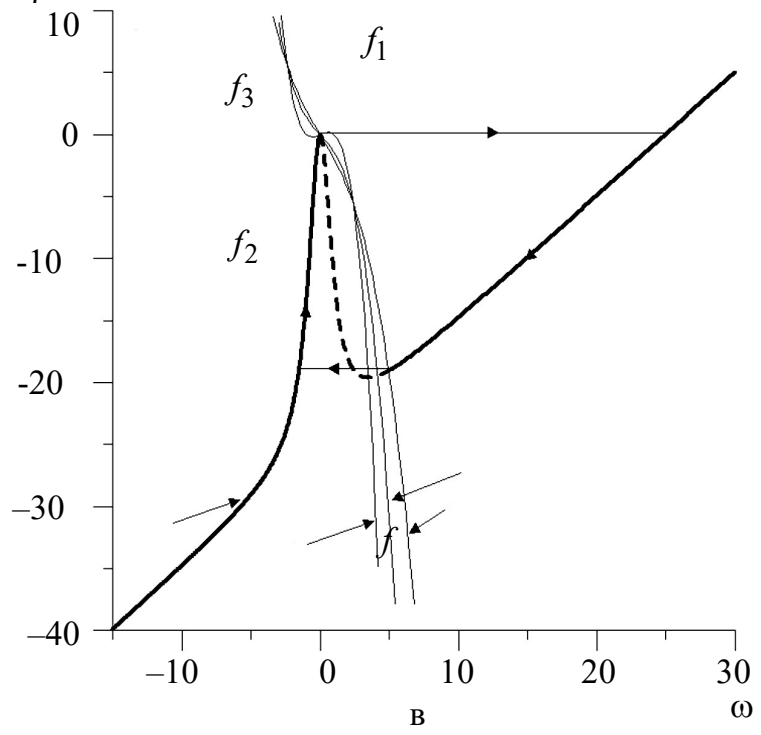

Рис. 3.2. Окончание.

Для правого возрастающего участка возможны два случая: 1) все его точки устойчивы (рис. 3.2,б); 2) часть возрастающего участка, примыкающая к минимуму, соответствует неустойчивым состояниям равновесия (рис. 3.2,в). Параметры того и другого случаев получаем из оценки расположения точки пересечения кривых $f_{1}, f_{2}, f_{3}$ относительно кривой $f=f(\omega)$. Если эта точка располагается ниже кривой, то условия устойчивости выполняются для всех точек возрастающего участка. Если эта точка располагается выше кривой, то часть возрастающего участка будет неустойчивой. Таким образом, неравенство $f\left(\omega_{1}\right)<f_{2}\left(\omega_{1}\right)$ определяет второй случай, а обратное неравенство - первый. В явном виде для параметров системы это неравенство выглядит как $\Lambda<-\frac{8 \sigma^{2}}{(\sigma-2)^{2}} \sqrt{\frac{\sigma-2}{3 \sigma+2}}$.

В системе имеется два типа бифуркаций состояний равновесия: 1) бифуркация слияния состояний равновесия - образование седлоузла и его исчезновение в точках экстремума функции $f=f(\omega)$; 2) обратная бифуркация Андронова - Хопфа для состояний равно- 
весия, соответствующих правой возрастающей ветви $f=f(\omega)$, когда неустойчивый предельный цикл, рождающийся от петли сепаратрисы седлового состояния равновесия «влипает» в устойчивое состояние равновесия, передавая ему свою неустойчивость.

Кроме состояний равновесия, других предельных множеств траекторий в системе (3.6) не обнаружено. Динамика рассматриваемой системы достаточно проста. Опишем ее поведение при изменении движущего момента двигателя. Допустим, что постоянный движущий момент двигателя изменяется квазистатически в сторону увеличения, а ХВ соответствует рис. 3.2,б. При этом частота вращения ротатора (ротора двигателя) плавно увеличивается, причем скорость изменения замедляется по мере приближения к собственной частоте колебательной системы, так как энергия, подводимая к двигателю, расходуется более на раскачку колебательной системы, чем на увеличение частоты вращения. Когда движущий момент достигает значения, соответствующего точке максимума кривой $f=f(\omega)$, частота ротатора скачком увеличивается до значения, соответствующего правой возрастающей ветви. При обратном изменении момента (торможении двигателя) скачок частоты вращения происходит с минимума кривой $f=f(\omega)$. Таким образом, в системе наблюдается классический эффект Зоммерфельда. Отличие случая, изображенного на рис. 3.2,в, только в том, что при торможении двигателя скачок частоты вращения осуществляется не с минимума $f=f(\omega)$, а из точки возрастающего участка этой кривой: имеется обрезанная гистерезисная петля.

\section{2. Хаотическая динамика простого вибрационного механизма}

Рассмотрим динамику простого вибрационного механизма, изображенного на рис. 3.3. Тело массой $m$ находится на круговой ленте. Оно связано с жесткой стенкой упругой связью, имеющей коэффициент упругости $c$, а также демпфером вязкого трения с коэффициентом $k$. На валу асинхронного электродвигателя перпен- 
дикулярно валу насажен кривошип радиуса $r_{1}$, который связан с телом упругой связью с коэффициентом $c_{1}$. Радиус кривошипа достаточно мал, и деформация упругой связи рассматривается только в горизонтальном направлении. Трение между телом и лентой является вязким с коэффициентом $q$. Ведущий шкив ленты разбаланси-

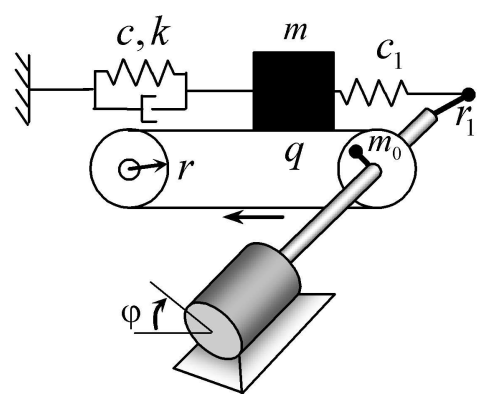

Рис. 3.3. Схема вибрационного механизма

рован грузом массы $m_{0}$, закрепленным на шкиве под углом $\varphi_{0}$ относительно кривошипа.

Модификации этой системы издавна используются для иллюстрации различного типа колебаний [1, 8, 33, 34]. Ниже, проведя аналитическое исследование системы в рамках разработанных приемов, мы расширим представления о ее динамике: продемонстрируем существование хаотических колебаний системы, а также механизмы рождения динамического хаоса [35].

При названных выше условиях уравнения движения системы имеют вид:

$$
\ddot{x}+\omega_{0}^{2} x=\frac{c_{1} r_{1}}{m} \sin \varphi+\frac{q r}{m} \dot{\varphi}-\frac{q+k}{m} \dot{x},
$$

$$
I \ddot{\varphi}=\widetilde{M_{d}}(\dot{\varphi})-r q(r \dot{\varphi}-\dot{x})+c_{1} r_{1}\left(x-r_{1} \sin \varphi\right) \cos \varphi-M_{0} \cos \left(\varphi+\varphi_{0}\right) \text {. }
$$

Здесь $\omega_{0}^{2}=\left(c+c_{1}\right) / m, I-$ приведенный момент инерции ротора, $\widetilde{M_{d}}(\dot{\varphi})$ - движущий момент двигателя, включая момент сил сопротивлений движению ротора, $M_{0}=m_{0} g e, e$-эксцентриситет.

Будем считать, что переменные, параметры и время в уравнениях (3.8) безразмерны. Рассматриваем систему при условиях на параметры: $I^{-1}=\mu \ll 1, \mu-$ малый параметр, $(q+k) / m=2 \mu h$ (диссипация в «колебательной» части системы достаточно мала), $c_{1} r_{1} / m=2 \mu \lambda \omega_{0}, \quad c_{1} r_{1}=2 \mu b \omega_{0}$. На другие комбинации параметров условия «малости» не распространяются. Как всегда, полагаем 
$\widetilde{M_{d}}(\dot{\varphi})=M_{d}-\delta \dot{\varphi}$, где $M_{d}-$ постоянная составляющая момента, $\delta$ - обобщенный коэффициент сопротивления.

В указанной области параметров динамическая система (3.8) является примером системы ротатора с колебательной нагрузкой. Рассматриваем зону параметров главного резонанса и действуем так же, как в разделе 3.1. В данном случае резонансная зона определена уравнением $M_{d}-\left(\delta+r^{2} q\right) \omega_{0}=\mu \Delta$.

Переход от системы (3.8) к эквивалентной системе в стандартной форме

$$
\begin{aligned}
& \dot{\theta}=\mu F_{1}(\theta, \eta, \varphi, \xi), \\
& \dot{\eta}=\mu F_{2}(\theta, \eta, \varphi, \xi), \\
& \dot{\xi}=\mu F_{3}(\theta, \eta, \varphi, \xi), \\
& \dot{\varphi}=\omega_{0}+\mu \Phi(\theta, \eta, \varphi, \xi)
\end{aligned}
$$

осуществляем через замену переменных:

$$
\begin{gathered}
x=q r / m \omega_{0}+\theta \sin \varphi+\eta \cos \varphi, \\
\dot{x}=(\theta \cos \varphi-\eta \sin \varphi) \omega_{0}, \\
\Phi=r q(\theta \sin \varphi+\eta \cos \varphi)-M_{0} / \omega_{0} \sin \left(\varphi+\varphi_{0}\right)+\xi .
\end{gathered}
$$

В системе (3.9)

$$
\begin{gathered}
F_{1}=\eta \Phi+1 / \omega_{0} X(.) \cos \varphi, \\
F_{2}=-\theta \Phi-1 / \omega_{0} X(.) \sin \varphi, \\
X(.)=2 \lambda \omega_{0} \sin \varphi+q r / m \Phi-2 h \omega_{0}(\theta \cos \varphi-\eta \sin \varphi), \\
F_{3}=-\left(\frac{\partial \Phi}{\partial \theta} F_{1}+\frac{\partial \Phi}{\partial \eta} F_{2}+\frac{\partial \Phi}{\partial \varphi} \Phi\right)-\left(\delta+q r^{2}\right) \Phi+ \\
+2 b \omega_{0}\left(\theta \sin \varphi+\eta \cos \varphi-r_{1} \sin \varphi\right) \cos \varphi .
\end{gathered}
$$

Усредняя уравнения (3.9) по быстрой фазе [36], получаем укороченную систему вида 


$$
\begin{aligned}
& \dot{\xi}=\mu\left(-b_{1} \xi+b_{2} \theta+b_{3} \eta+\Delta\right), \\
& \dot{\theta}=\mu\left(-b_{4} \theta+b_{5} \eta+\eta \xi+b_{6}\right), \\
& \dot{\eta}=\mu\left(-b_{4} \eta-b_{5} \theta-\theta \xi+b_{7}\right), \\
& \dot{\varphi}=\omega_{0}+\mu \xi,
\end{aligned}
$$

где $b_{1}=q r^{2}+\delta, \quad b_{2}=-\frac{M_{0} r q}{2 \omega_{0} \sin \varphi_{0}}, \quad b_{3}=b \omega_{0}+\frac{M_{0} r q}{2 \omega_{0} \cos \varphi_{0}}, \quad b_{4}=h$, $b_{5}=\frac{(q r)^{2}}{2 m \omega_{0}}, b_{6}=-\frac{M_{0} r q}{2 m \omega_{0}^{2} \sin \varphi_{0}}, b_{7}=\frac{M_{0} r q}{2 m \omega_{0}^{2} \cos \varphi_{0}-\lambda}$.

В свою очередь, первые три уравнения (3.10) заменой переменных и времени

$$
\begin{aligned}
& x=b_{4}^{-1}\left(\xi+b_{5}\right), \quad y=\left(b_{3} \eta+b_{2} \theta\right) / b_{1} b_{4}-\Lambda, \\
& z=\left(b_{3} \theta-b_{2} \eta\right) / b_{1} b_{4}+R, \mu b_{4} \tau=\tau_{n}
\end{aligned}
$$

сводятся к системе

$$
\begin{aligned}
& \dot{x}=-\sigma(x-y)+\rho, \\
& \dot{y}=-y+R x-x z, \\
& \dot{z}=-z+x y+\Lambda x,
\end{aligned}
$$

где

$$
\begin{gathered}
\sigma=b_{1} / b_{4}=\left(q r^{2}+\delta\right) / h, \quad \rho=\frac{\Delta+\left(b_{1} b_{4} b_{5}+b_{3} b_{7}+b_{2} b_{6}\right) / b_{4}}{b_{4}^{2}}, \\
R=\frac{b_{2} b_{7}-b_{3} b_{6}}{b_{1} b_{4}^{2}}=\frac{2 A \lambda}{\left(\delta+q r^{2}\right) h^{2}} \sin \varphi_{0}, \quad A=\frac{M_{0} q r}{2 \omega_{0}} \\
\Lambda=\frac{b_{3} b_{7}+b_{2} b_{6}}{b_{1} b_{4}^{2}}=\frac{\lambda\left(A^{2}-b^{2} \omega_{0}^{2}\right)}{b \omega_{0}\left(\delta+q r^{2}\right) h^{2}} .
\end{gathered}
$$

Как всегда, нас интересует исследование различных типов предельных движений в усредненной системе и связанные с ними качественные картины характеристики вращения в резонансной зоне параметров. В силу замен переменных и параметров $M_{d} \sim \Delta \sim \rho$, $\xi(t) \sim x(t)$ (эти параметры и переменные связаны линейно, как 
и в разделе 3.1$)$, т. е. и в этом случае картину ХВ мы можем заменить картиной зависимости $\rho=\rho\left(\overline{x^{*}\left(t, t_{0}\right)}\right)$, имеющей те же самые качественные особенности.

Приведем свойства системы (3.11), некоторые из них частично совпадают со свойствами системы из раздела 3.1 .

1. Система (3.11) является диссипативной. Это свойство устанавливается при помощи квадратичной формы $V=\frac{1}{2}\left(x^{2}+\right.$ $\left.+(y+\Lambda)^{2}+(z-\sigma-R)^{2}\right)$. Ее производная вдоль траекторий системы имеет вид $\dot{V}=-\sigma x^{2}-(\rho-\sigma \Lambda) x-y^{2}-\Lambda y-z^{2}+(\sigma+R) z$. Очевидно, что эта квадратичная форма отрицательна для некоторого шара $V \leq L^{2}$, т. е. все предельные множества фазовых траекторий системы (3.11) ограничены шаром диссипации.

2. Система (3.11) в зависимости значений от параметров имеет от одного до трех состояний равновесия, координаты которых

$$
x_{0}=\omega, y_{0}=\frac{R \omega-\Lambda \omega^{2}}{1+\omega^{2}}, z_{0}=\frac{R \omega^{2}+\Lambda \omega}{1+\omega^{2}},
$$

где $\omega_{1,2,3}$ являются решениями уравнения

$$
f=\omega+\frac{\Lambda \omega^{2}-R \omega}{1+\omega^{2}}, f=\rho / \sigma .
$$

Как и ранее, параметр $\omega$ имеет смысл расстройки частоты периодических вращений ротатора и собственной частоты осциллятора.

3. Если система (3.11) имеет одно состояние равновесия $O\left(x_{0}, y_{0}, z_{0}\right)$, то оно глобально асимптотически устойчиво. Свойство доказывается при помощи функции Ляпунова $V=\frac{1}{2}\left(m x_{1}^{2}+y_{1}^{2}+z_{1}^{2}\right)$, где $x_{1}=x-x_{0}, y_{1}=y-y_{0}, z_{1}=z-z_{0}$. Ее производная вдоль траекторий системы имеет вид $\dot{V}=-\left(\alpha_{1} x_{1}+y_{1}\right)^{2}-\left(\alpha_{2} x_{1}+z_{1}\right)^{2} \leq 0$, $\forall\left(x_{1}, y_{1}, z_{1}\right)$, где $\alpha_{1}=\frac{\sigma m+R-z_{0}}{2}, \alpha_{2}=-\frac{y_{0}+\Lambda}{2}$, а $m-$ положи- 
тельный корень уравнения $\sigma^{2} m^{2}+2 \sigma\left(R-z_{0}-2\right) m+\left(R-z_{0}\right)+$ $+\left(y_{0}+\Lambda\right)^{2}=0$. Нетрудно установить, что условия существования положительного корня этого уравнения и условия единственности состояния равновесия в системе (3.11) (условия взаимооднозначности функции $f$ ) совпадают.

4. Свойства состояний равновесия. Характеристическое уравнение для произвольного состояния равновесия $O\left(x_{0}, y_{0}, z_{0}\right)$ системы (3.11) имеет вид $p^{3}+a_{0} p^{2}+a_{1} p+a_{2}=0$, где $a_{0}=\sigma+2$, $a_{1}=2 \sigma+1+\omega^{2}+\sigma \frac{\Lambda \omega-R}{1+\omega^{2}}, \quad a_{2}=\sigma\left(1+\omega^{2}\right)\left(1+\frac{R \omega^{2}+2 \Lambda \omega-R}{\left(1+\omega^{2}\right)^{2}}\right)$. При $\omega=0 \quad(\rho=0)$ условием устойчивости состояния равновесия $O(0,0,0)$ является неравенство $R<1$. При $\omega \neq 0$ условия Гурвица эквивалентны неравенствам

$$
\begin{aligned}
& a_{1}>0: f>(<) f_{1}, a_{2}>0: f>(<) f_{2}, \\
& a_{0} a_{1}-a_{2}>0: f>(<) f_{3}, \omega>0(\omega<0),
\end{aligned}
$$

где $f_{1}=-\omega\left(\sigma+1+\omega^{2}\right) / \sigma, \quad f_{2}=-\omega\left(R-1+\omega^{2}\right) / 2, \quad f_{3}=-\omega\left(\sigma^{2}+\right.$ $\left.+4 \sigma-\sigma R+2+2 \omega^{2}\right) / \sigma^{2}$. Другими словами, условием устойчивости любого состояния равновесия системы является расположение соответствующей ему точки кривой (3.12) выше (ниже) всех кривых $f_{1,2,3}$ при $\omega>0(\omega<0)$. Уравнения $f=f_{1,2,3}, \quad f=\rho / \sigma$ определяют в пространстве параметров системы бифуркационные поверхности, для точек которых происходят изменения структуры фазовых траекторий на локальных многообразиях состояний равновесия, а также изменения их устойчивости.

Как и в разделе 3.1, имеется пара полезных свойств вспомогательных функций:

a) $f_{2}$ пересекает $f$ в начале координат и в точках экстремума; 
б) все кривые, кроме нуля, пересекаются между собой в двух точках $\omega_{1,2}= \pm \sqrt{\frac{3 \sigma-\sigma R+2}{\sigma-2}}$.

Этими свойствами мы воспользуемся при анализе устойчивости состояний равновесия.

5. Хаотические аттракторы.

5.1. Аттрактор Лоренца. При $\rho=\Lambda=0$ уравнения (3.11) являются известной системой Лоренца [37]. Эта ставшая классической система при определенных значениях параметров $\sigma=\sigma^{*}$, $R=R^{*}>R_{c}, R_{c}=\frac{\sigma^{2}+4 \sigma}{\sigma-2}$ имеет в фазовом пространстве $G(x, y, z)$ единственное притягивающее множество - странный аттрактор (Лоренца). Заметим, что в соответствии с п. 4 выражение для $R_{c}$ определяется из условия совпадения нулей функций $f$ и $f_{3}$. Это условие соответствует потере устойчивости состояний равновесия $O_{2,3}( \pm \sqrt{R-1}, \pm \sqrt{R-1}, R-1)$ от «влипания» в них седловых предельных циклов, родившихся ранее (по параметру $R$ ) от петли сепаратрисы седла $O_{1}(0,0,0)$. Если точнее, то странный аттрактор существует уже при $R^{*}<R<R_{c}$, где $R^{*}$ - значение параметра $R$, соответствующее петле сепаратрисы седла. В этом случае странный аттрактор и устойчивые состояния равновесия существуют совместно, имея непересекающиеся области притяжения. В этом интервале значений параметра $R$ в зависимости от начальных условий системы может реализоваться или странный аттрактор, или одно из двух устойчивых состояний равновесия [37].

«Деформация» и вырождение аттрактора Лоренца при ненулевых параметрах $\rho$ и $\Lambda$ исследовалась в численном эксперименте. При $\Lambda=0$ и увеличении параметра $\rho$ от нуля аттрактор теряет симметрию, так что изображающая (фазовая) точка проводит большее время в окрестности правого седло-фокуса (если рассматривать проекцию аттрактора на плоскость $(x, z))$. Затем, с рождением седлового предельного цикла, это состояние равновесия приобретает устойчивость. В этом случае в зависимости от начальных условий может быть реализовано как состояние равновесия, так 
и хаотический аттрактор. При дальнейшем увеличении $\rho$ седловой цикл «влипает» в петлю сепаратрисы седла, а состояние равновесия становится ГАУ. В силу инвариантности системы (3.11) относительно замены $(x, y, z) \mapsto(-x,-y, z), \rho \mapsto-\rho$ тот же сценарий вырождения лоренцевского динамического хаоса наблюдается при изменении параметра $\rho$ в отрицательную сторону с той лишь разницей, что в конечном итоге становится ГАУ правое состояние равновесия. Описанный сценарий имеет место и при ненулевых значениях параметра $\Lambda$, по крайней мере, для всех значений, для которых $|\Lambda|<3$. Асимметричные аттракторы Лоренца показаны на рис. 3.4,а,в. Заметим, что при выполнении неравенства $\rho \Lambda>0$ и небольших значениях $|\Lambda|$ существует $\rho$, при котором аттрактор имеет «визуальную» симметрию (рис. $3.4,6)$.

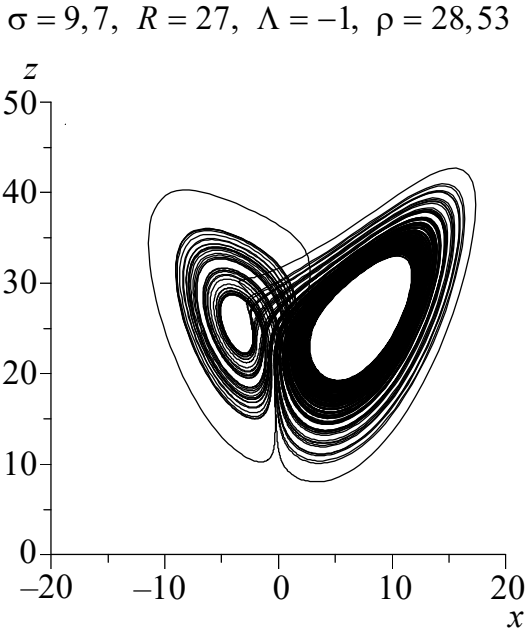

a

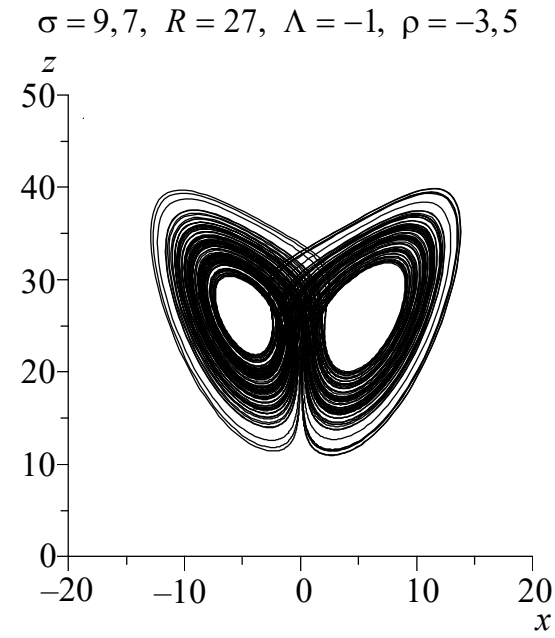

б

Рис. 3.4. Странный аттрактор, асимметричный «вправо» (a); симметричный аттрактор Лоренца (б); странный аттрактор, асимметричный «влево» (в) (окончание рис. см. на с. 86). 


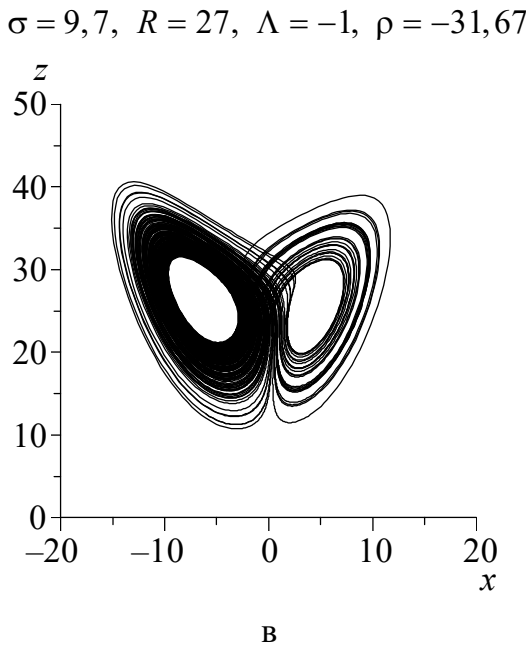

Рис. 3.4. Окончание.

5.2. Аттрактор Фейгенбаума и перемежаемость. Этот тип хаотических аттракторов обнаружен при условии $\rho \Lambda>0$ и достаточно больших абсолютных значениях параметра $\Lambda$. В эксперименте $|\Lambda| \geq 7$. Аттрактор Фейгенбаума [38] показан на рис. 3.5,а. Если $\Lambda \leq-7$ (в эксперименте), то при увеличении параметра $\rho$ левое состояние равновесия системы (3.11) теряет устойчивость с рождением устойчивого предельного цикла. Далее этот цикл претерпевает каскад бифуркаций удвоения периода. В некотором интервале значений $\rho$ родившийся хаотический аттрактор имеет область притяжения, изолированную от областей притяжения других предельных множеств. При дальнейшем увеличении $\rho$ область притяжения аттрактора пересекается с областью притяжения другого хаотического предельного множества (в эксперименте не уточнялось, какого: вероятно, это множество является некоторым «наследством» асимметричного аттрактора Лоренца). В результате имеется типичная картина перемежаемости [24] (рис. 3.5,б). На рисунке показан отрезок траектории только с одним выбросом из области притяжения аттрактора Фейгенбаума. В силу инвариантности системы (3.11) относительно преобразования $\quad(x, y, z) \mapsto(-x,-y, z), \quad \rho \mapsto-\rho$, 
$\Lambda \mapsto-\Lambda$ та же картина наблюдается в правой полуплоскости $(x, z)$ при положительных $\Lambda$ и уменьшении $\rho$.

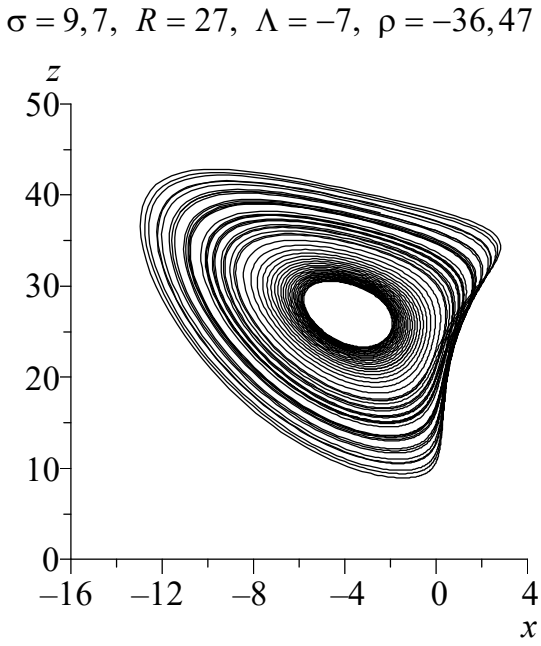

a $\sigma=9,7, R=27, \Lambda=-7, \rho=-36,27$

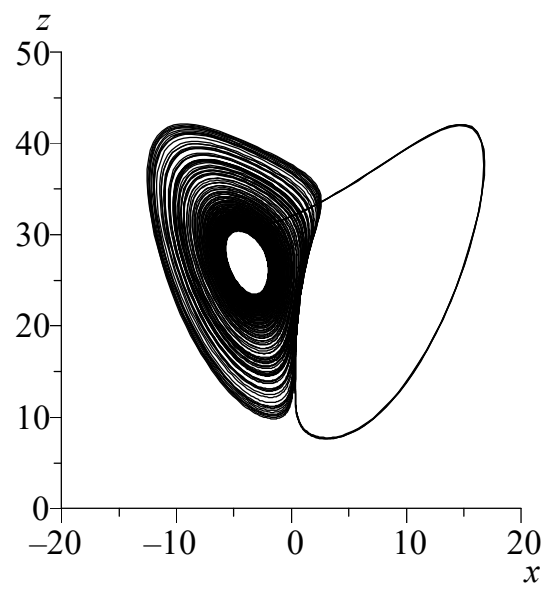

6

Рис. 3.5. Аттрактор Фейгенбаума (а), перемежаемость аттракторов (б).

При отрицательных значениях параметра $R$ и любых значениях остальных параметров существование хаотических аттракторов в системе (3.11) не обнаружено.

Численный эксперимент. Как всегда, численный эксперимент проводим для иллюстрации аналитических результатов и уточнения деталей.

В эксперименте исследовалось точечное отображение Пуанкаpe секущей гиперплоскости $\varphi=$ const в себя через период $2 \pi$, точнее, $(\theta, \eta, \xi)_{\varphi=\varphi_{0}} \rightarrow(\bar{\theta}, \bar{\eta}, \bar{\xi})_{\varphi=\varphi_{0}+2 \pi}$. Для вращательных движений секущая $\varphi=$ const является глобальной. Из соображений удобства отображение строилось для эквивалентной (3.8) системы вида 


$$
\begin{aligned}
& \dot{\theta}=\mu\left(\eta \xi+\left(2 \lambda \sin \varphi+\frac{q r}{m \omega_{0}} \xi-2 h(\theta \cos \varphi-\eta \sin \varphi)\right) \cos \varphi\right), \\
& \dot{\eta}=\mu\left(-\theta \xi-\left(2 \lambda \sin \varphi+\frac{q r}{m \omega_{0}} \xi-2 h(\theta \cos \varphi-\eta \sin \varphi)\right) \sin \varphi\right), \\
& \dot{\xi}=M_{d}-\left(\delta+q r^{2}\right) \omega_{0}-\mu\left(\delta+q r^{2}\right) \xi+r q \omega_{0}(\theta \cos \varphi-\eta \sin \varphi)+ \\
& +c_{1} r_{1}\left(\frac{r q}{m \omega_{0}}+\theta \sin \varphi+\eta \cos \varphi-r_{1} \sin \varphi\right) \cos \varphi-M_{0} \cos \left(\varphi+\varphi_{0}\right), \\
& \dot{\varphi}=\omega_{0}+\mu \xi .
\end{aligned}
$$

При переходе от системы (3.8) к системе (3.13) выполнена замена

$$
\begin{aligned}
& x=q r / m \omega_{0}+\theta \sin \varphi+\eta \cos \varphi, \\
& \dot{x}=(\theta \cos \varphi-\eta \sin \varphi) \omega_{0}, \quad \dot{\varphi}=\omega_{0}+\mu \xi,
\end{aligned}
$$

где $\mu=I^{-1}$.

Для сравнения аналитического и численного результатов значения параметров системы (3.13) выбирались так, чтобы их комбинации в качестве параметров усредненной системы были близки к тем, при которых изображены аттракторы на рис. 3.4, 3.5.

На рис. 3.6,a изображен асимметричный аттрактор Лоренца в пространстве отображения Пуанкаре. Некоторое «визуальное» различие с рис. 3.4,a связано с преобразованием координат. При указанных параметрах исходной системы параметры усредненной системы имеют значения $\sigma=9,9, \quad R=27,39, \Lambda=-0,87$. На рис. 3.6,б показан отрезок хаотической вращательной траектории на развертке фазового цилиндра, соответствующей этому хаотическому аттрактору.

На рис. 3.7,а показан аттрактор Фейгенбаума на базе удвоений периода инвариантного тора. При заданных параметрах системы параметры усредненной системы (3.11) имеют значения $\sigma=9,9$, $R=27,39, \Lambda=-7,061$. На рис. 3.7,б показан отрезок хаотической вращательной траектории на развертке фазового цилиндра. 

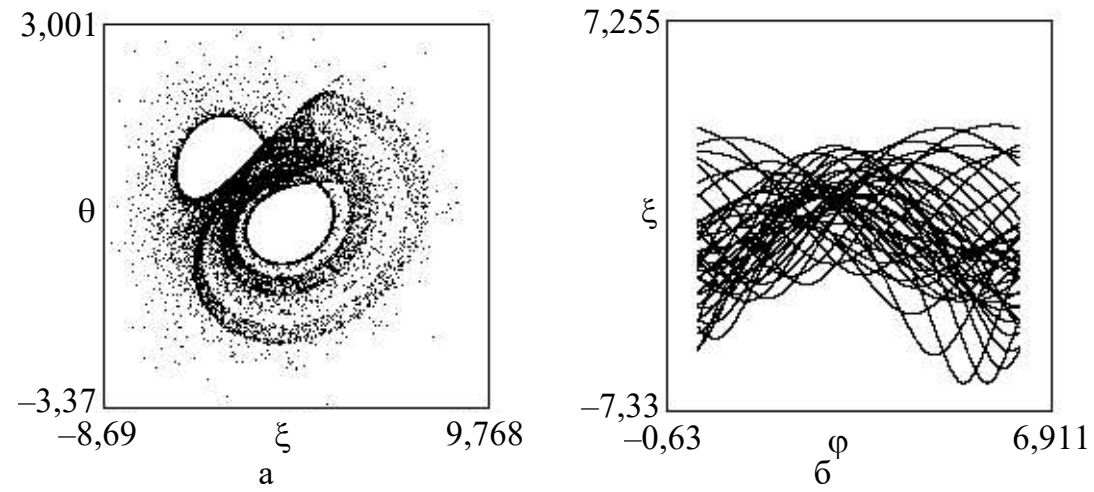

Рис. 3.6. Асимметричный «вправо» аттрактор Лоренца в пространстве точечного отображения (a); отрезок хаотической траектории в проекции на развертку фазового цилиндра (б).
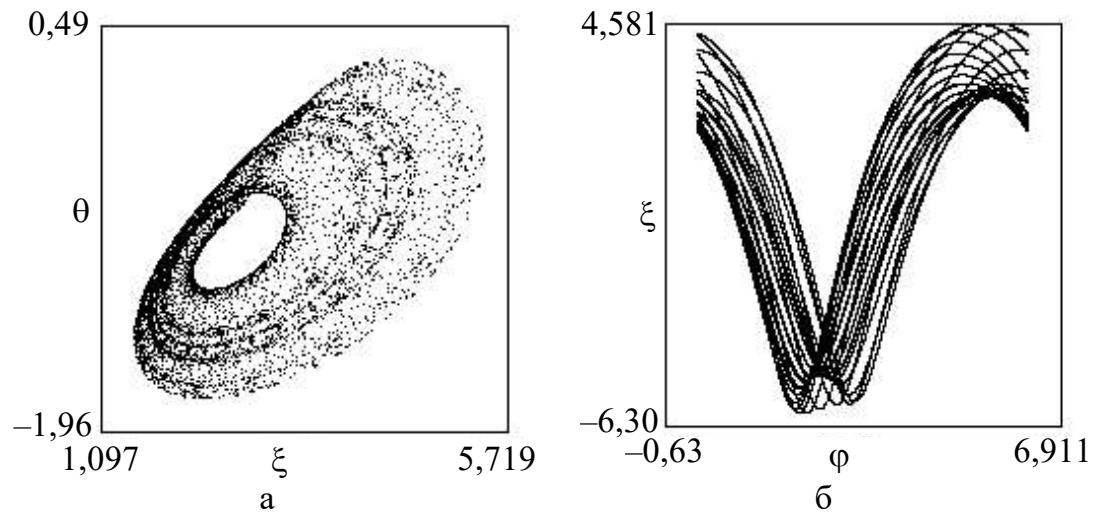

Рис. 3.7. Аттрактор Фейгенбаума в пространстве точечного отображения (а); отрезок фазовой траектории в проекции на развертку фазового цилиндра (б).

Обобщая полученную информацию, представим галерею качественных картин ХВ в резонансной зоне.

1) Допустим, что $R \leq 0$. В этом случае динамика системы достаточно проста. В системе (3.11) существуют только состояния равновесия - вращательные предельные циклы в системе (3.8). Состояния равновесия испытывают только один тип бифуркаций слияние состояний равновесия с образованием седло-узла и после- 
дующим его исчезновением. В этом случае при разных параметрах $\mathrm{XB}$ имеют или две или одну гистерезисных петли. Срыв частоты вращения ротатора происходит с ее экстремумов. На рис. 3.8 изображена двухпетлевая XВ (жирная линия). Пунктирные ее части соответствуют неустойчивым состояниям равновесия типа седло или седло-фокус, сплошные - устойчивым состояниям равновесия системы (3.11) (устойчивым предельным циклам системы (3.8)). Тонкими линиями изображены вспомогательные функции, определяющие устойчивость и тип состояний равновесия [39]. В данном случае можно говорить о «двойном» эффекте Зоммерфельда.

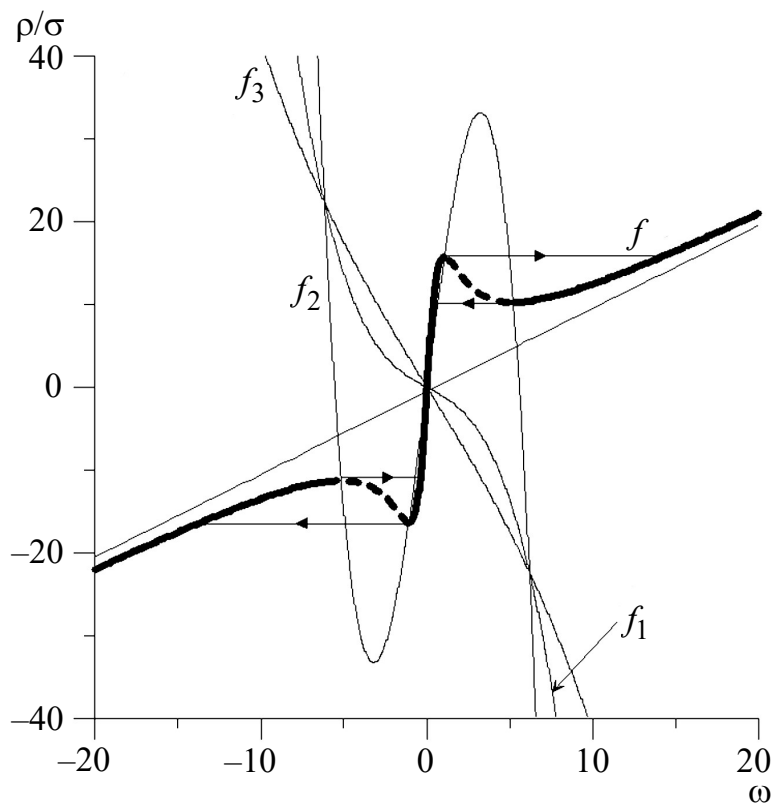

Рис. 3.8. Характеристика вращения в резонансной зоне:

$$
\sigma=15, R=-30, \Lambda=-0,5
$$

При уменьшении $|R|$, а также при увеличении $|\Lambda|$ исчезает левая и остается только правая гистерезисная петля (рис. 3.9). В результате получаем классический (однопетлевой) эффект Зоммерфельда. В силу инвариантности системы (3.11) относительно заме- 
ны $(x, y, z) \mapsto(-x,-y, z), \rho \mapsto-\rho, \Lambda \mapsto-\Lambda$ при увеличивающихся положительных $\Lambda$ исчезает правая и остается только левая гистерезисная петля.

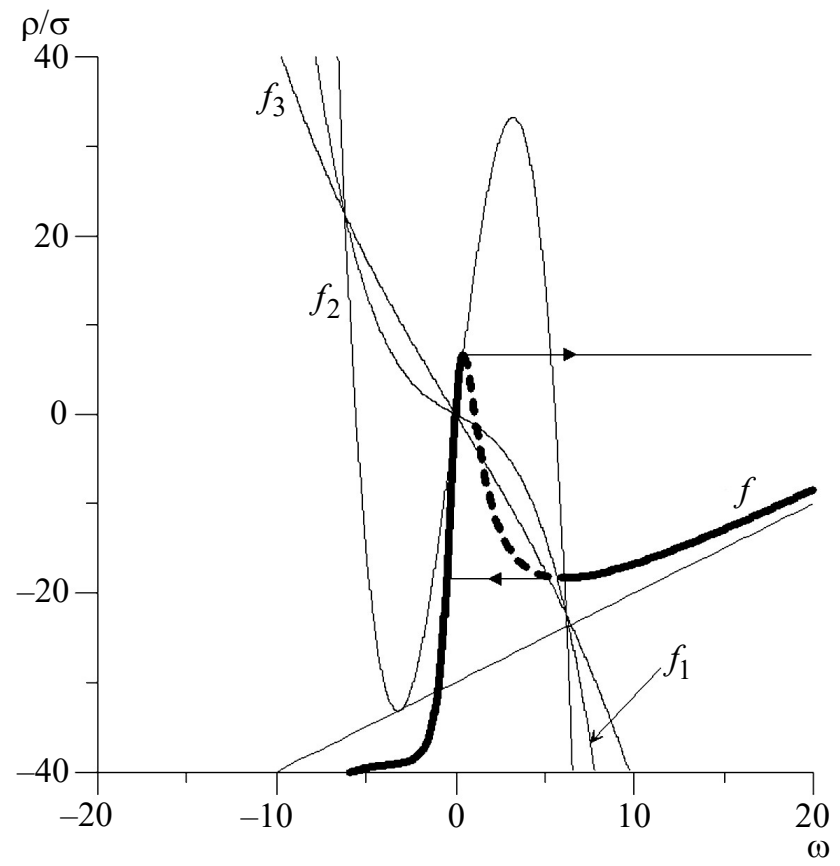

Рис. 3.9. Характеристика вращения в резонансной зоне:

$$
\sigma=15, R=-30, \Lambda=-30
$$

2) Допустим, что $R>0$. В этом случае динамика системы более разнообразна при изменении параметров системы, следовательно, разнообразнее и набор качественных картин характеристики вращения. Остановимся только на случаях существования в системе хаотических аттракторов.

Допустим, что $R^{*}<R<R_{c}$ (рис. 3.10,a). При этом в некотором интервале значений параметра $\rho / \sigma$ существуют два устойчивых состояния равновесия и аттрактор Лоренца (на рисунке эта область показана штриховкой). В заштрихованной области в зависимости от начальных условий системы может быть реализовано как одно, 
так и другое состояния равновесия (периодические движения системы (3.8)), а также странный аттрактор, соответствующий хаотическому поведению мгновенной частоты ротатора. Вне зоны наблюдается режим периодических вращений ротатора, который реализуется при любых начальных условиях.
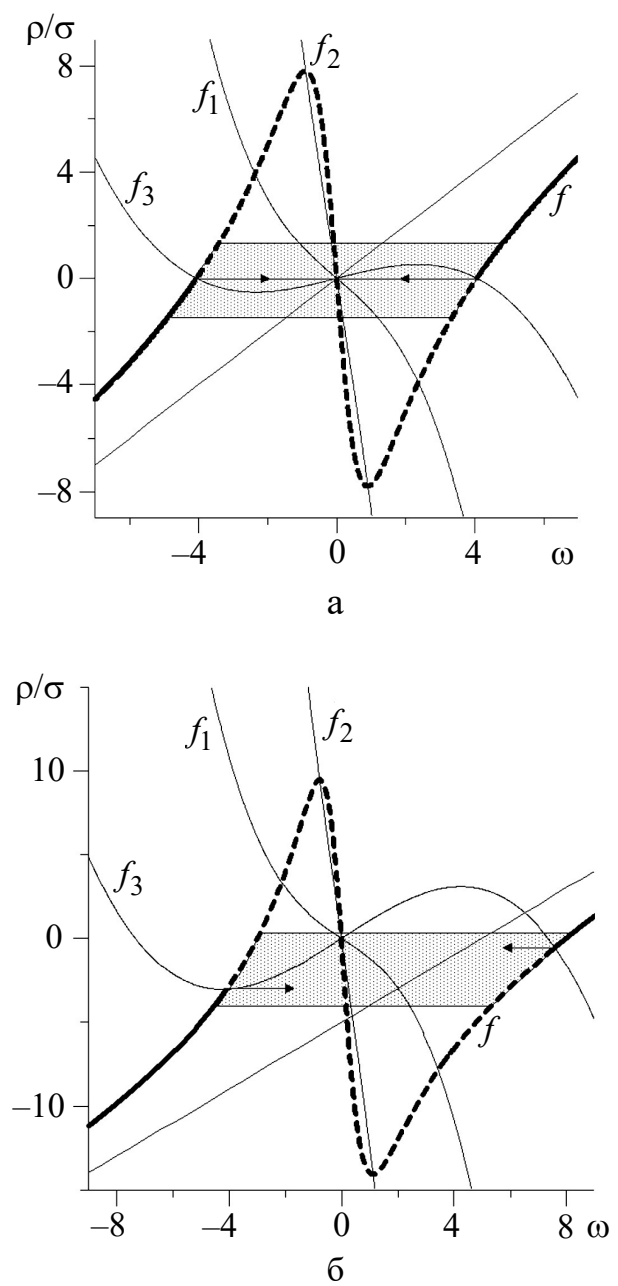

Рис. 3.10. Характеристика вращения в резонансной зоне: $\sigma=10, R=17,5, \Lambda=0$ (a); $\sigma=10, R=25, \Lambda=-5$ (б). 
На рис. 3.10,б изображена характеристика вращения при $R>R_{c}$. В заштрихованной зоне при любых начальных условиях системы реализуется странный аттрактор, свойства которого изменяются в зависимости от параметра $\rho / \sigma$. При этом изменяется и временное среднее $\left\langle x\left(t, t_{0}\right)\right\rangle_{t}$. По причине сильной зависимости траекторий аттрактора от начальных условий и конечности интервала усреднения (реальные измерения) это среднее будет существенно зависеть от начального момента $t_{0}$. Из сказанного можно сделать следующие выводы относительно характеристики вращения в заштрихованной зоне:

а) XВ в заштрихованной зоне является невоспроизводимой: при квазистатическом увеличении параметра $\rho / \sigma$ (движущего момента двигателя) получается одна кривая (ветвь), а при обратном изменении (сколь угодно медленном) - совершенно другая;

б) характер XВ в заштрихованной зоне сложный: она имеет бесконечное множество перепутанных между собой ветвей, «растущих» от точек срыва с периодических на хаотические вращения ротатора, соответствующих на рис. 3.10 концам сплошных линий.

В заключение разъясним, почему дисбаланс ротора является причиной того, что в этой популярной демонстрационной установке может наблюдаться или не наблюдаться хаотический динамический режим. При отсутствии дисбаланса ротора асинхронного двигателя его уравнение движения является фактически уравнением первого порядка относительно переменной $\dot{\varphi}$ - частоты вращения. То есть асинхронный двигатель не представляет собой «полноценный» ротатор, под которым мы понимаем динамическую систему с одной степенью свободы. В этом случае формальное усреднение системы (3.8) даст систему второго порядка, а это значит, что все динамические режимы системы являются регулярными. То же самое произойдет и при незначительном дисбалансе ротора. По этой причине динамический хаос в системе с хорошо сбалансированным ротором не наблюдается.

Обратим внимание на следующий факт. Параметр системы $R=\frac{2 A \lambda}{\left(\delta+q r^{2}\right) h^{2}} \sin \varphi_{0}, A=\frac{M_{0} q r}{2 \omega_{0}}$, существенно зависит от угла крепления дисбаланса относительно кривошипа: при одних углах 
крепления может наблюдаться хаотическая динамика механизма, а при других углах вместо хаоса можно получить, наоборот, стабилизацию вращений. В частности, если параметр $R<0$ и при этом велик по абсолютной величине, то участок межу гистерезисными петлями может быть весьма крутым (см. рис. 3.8). То есть при соответствующем выборе величины и точки подвеса дисбаланса можно добиться существенной стабилизации вращений двигателя, когда внешние, в том числе и случайные, воздействия не будут оказывать заметных отклонений в частоте вращения исполнительного механизма. Здесь мы получаем определенный аналог со случаем принудительной синхронизации ротатора силовым воздействием.

\section{3. Динамика связанных ротаторов}

Модель «связанные ротаторы» представляется дифференциальными уравнениями вида $[40,41]$

$$
\begin{aligned}
& I \ddot{\varphi}_{1}+\delta_{1} \dot{\varphi}_{1}+\sigma_{1} \sin \varphi_{1}=\gamma_{1}+a_{1} \dot{\varphi}_{2}+b_{1} \sin \varphi_{2}, \\
& I \ddot{\varphi}_{2}+\delta_{2} \dot{\varphi}_{2}+\sigma_{2} \sin \varphi_{2}=\gamma_{2}+a_{2} \dot{\varphi}_{1}+b_{2} \sin \varphi_{1} .
\end{aligned}
$$

Система задана в тороидальном фазовом пространстве $G\left(\varphi_{1,2}, \dot{\varphi}_{1,2}\right)=T^{2} \times R^{2}$ и рассматривается в области параметров $D=\left\{I>0, \delta_{1,2}>0, \gamma_{1,2} \geq 0, \delta_{1} \delta_{2}-a_{1} a_{2}>0, \sigma_{1,2} \geq 0\right\}$.

В динамике системы (3.14) будут представлять интерес следующие задачи:

- определение областей параметров, соответствующих топологически различным типам движений системы: колебательным, колебательно-вращательным и вращательным движениям ротаторов;

- исследование качественных структур траекторий и бифуркаций вращательных движений;

- построение качественных картин ХВ ротаторов.

1. Динамика ротаторов и притягивающие множества траекторий системы (3.14).

Как показано в [40], в фазовом пространстве $G\left(\varphi_{1,2}, \dot{\varphi}_{1,2}\right)$ системы (3.14) существует тороидальная поглощающая область 


$$
\begin{gathered}
G^{*}\left(\varphi_{1,2}, \dot{\varphi}_{1,2}\right)=\left(\forall \varphi_{1,2}, \omega_{1,2}-\omega_{1,2}^{0} \leq \dot{\varphi}_{1,2} \leq \omega_{1,2}+\omega_{1,2}^{0}\right), \\
\omega_{1}=\frac{\gamma_{1} \delta_{2}+\gamma_{2} a_{1}}{\delta_{1} \delta_{2}-a_{1} a_{2}}, \omega_{2}=\frac{\gamma_{2} \delta_{1}+\gamma_{1} a_{2}}{\delta_{1} \delta_{2}-a_{1} a_{2}},
\end{gathered}
$$

в которую попадают все фазовые траектории системы и остаются в ней при $\tau \rightarrow \infty$. То есть для параметров из области $D$ все предельные множества траекторий системы находятся в поглощающей области фазового пространства $G^{*}$ и любое ее решение $\left(\varphi_{1}\left(\tau, \tau_{0}\right), \varphi_{2}\left(\tau, \tau_{0}\right)\right)$ имеет ограниченные производные $\dot{\varphi}_{1}\left(\tau, \tau_{0}\right)$, $\dot{\varphi}_{2}\left(\tau, \tau_{0}\right)$.

Разделим предельные множества фазовых траекторий в области $G^{*}$ на классы:

$K_{00}$ - класс траекторий, ограниченных по $\varphi_{1}$ и $\varphi_{2}$;

$K_{0 r}$ - класс траекторий, ограниченных по $\varphi_{1}$ и неограниченных по $\varphi_{2}$;

$K_{r 0}-$ класс траекторий, неограниченных по $\varphi_{1}$ и ограниченных по $\varphi_{2}$;

$K_{r r}$ - класс траекторий, неограниченных по $\varphi_{1}$ и $\varphi_{2}$.

Очевидно, что $\left\langle\dot{\varphi}_{1}^{*}\right\rangle_{\tau}=0,\left\langle\dot{\varphi}_{2}^{*}\right\rangle_{\tau}=0$, если $L^{*} \in K_{00} ;\left\langle\dot{\varphi}_{1}^{*}\right\rangle_{\tau} \neq 0$, $\left\langle\dot{\varphi}_{2}^{*}\right\rangle_{\tau}=0, \quad$ если $\quad L^{*} \in K_{r 0} ; \quad\left\langle\dot{\varphi}_{1}^{*}\right\rangle_{\tau}=0,\left\langle\dot{\varphi}_{2}^{*}\right\rangle_{\tau} \neq 0$, если $L^{*} \in K_{0 r} ;$ $\left\langle\dot{\varphi}_{1}^{*}\right\rangle_{\tau} \neq 0,\left\langle\dot{\varphi}_{2}^{*}\right\rangle_{\tau} \neq 0$, если $L^{*} \in K_{r r}$. Определение областей параметров, соответствующих различным классам неблуждающих траекторий, проведем методом двумерных систем сравнения [10].

Введем для каждого из уравнений (3.14) системы сравнения вида

$$
\begin{aligned}
& A_{1}^{ \pm}: I \ddot{\varphi}_{1}+\delta_{1} \dot{\varphi}_{1}+\sigma_{1} \sin \varphi_{1}=\delta_{1} \omega_{1}+\mu_{1}^{ \pm}, \\
& A_{2}^{ \pm}: I \ddot{\varphi}_{2}+\delta_{2} \dot{\varphi}_{2}+\sigma_{2} \sin \varphi_{2}=\delta_{2} \omega_{2}+\mu_{2}^{ \pm},
\end{aligned}
$$

где

$$
\delta_{1,2} \omega_{1,2}+\mu_{1,2}^{+}=\sup _{\forall\left(\varphi_{1,2}, \dot{\varphi}_{1,2}\right) \in G^{*}}\left(\gamma_{1,2}+a_{1,2} \dot{\varphi}_{1,2}+b_{1,2} \sin \varphi_{1,2}\right),
$$




$$
\begin{gathered}
\delta_{1,2} \omega_{1,2}+\mu_{1,2}^{-}=\inf _{\forall\left(\varphi_{1,2}, \dot{\varphi}_{1,2}\right) \in G^{*}}\left(\gamma_{1,2}+a_{1,2} \dot{\varphi}_{1,2}+b_{1,2} \sin \varphi_{1,2}\right), \mu_{1,2}^{ \pm}= \\
= \pm\left(\left|a_{1,2}\right| \omega_{1,2}^{0}+\left|b_{1,2}\right|\right) .
\end{gathered}
$$

Траектории систем сравнения определяют в $G\left(\varphi_{1,2}, \dot{\varphi}_{1,2}\right)$ поверхности без контакта для векторного поля системы (3.14). В этом нетрудно убедиться, рассматривая поворот векторного поля системы на траекториях систем сравнения. Это значит, что если обе системы $A_{k}^{+}$и $A_{k}^{-}$имеют топологически одинаковые структуры траекторий на развертках цилиндров $\left(\varphi_{k}, \dot{\varphi}_{k}\right)$, то проекции предельных множеств $K_{(.)}$на эти развертки оказываются лежащими между особыми многообразиями систем сравнения. На рис. 3.11 изображены сепаратрисы седел и предельные циклы систем сравнения, а стрелками указано направление составляющей векторного поля системы (3.14) на этих траекториях.

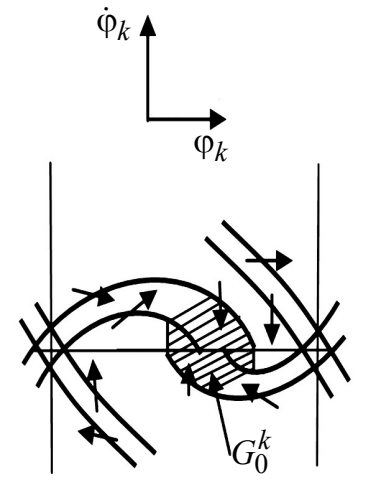

a

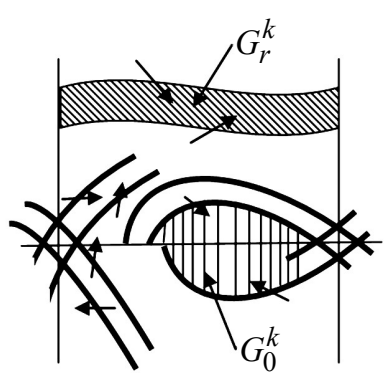

6

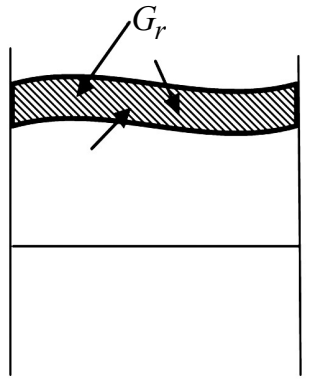

B

Рис. 3.11. Качественные картины траекторий систем сравнения и ориентация проекции векторного поля системы (3.14) на их особых траекториях.

Введем обозначения: $D_{(0) k}^{ \pm}, D_{(0 r) k}^{ \pm}, D_{(r) k}^{ \pm}, k=1,2$ - области параметров, для которых обе системы сравнения $A_{k}^{ \pm}$(отдельно при 
каждом индексе $k$ ) имеют, соответственно, глобально устойчивое состояние равновесия, устойчивое состояние равновесия и предельный цикл, глобально устойчивый предельный цикл (см. рис. 3.11, а также раздел 1.1). В плоскостях параметров $\left(\lambda_{k}, \gamma_{k}^{ \pm}\right), \quad$ где $\lambda_{k}=\delta_{k}\left(I \sigma_{k}\right)^{-1 / 2}, \gamma_{k}^{ \pm}=\frac{\delta_{k} \omega_{k}+\mu_{k}^{ \pm}}{\sigma_{k}}$, эти области отделяются прямой $\gamma_{k}^{ \pm}=1$ (бифуркация состояния равновесия) и кривой Трикоми $\lambda_{k}=\lambda_{k}^{*}\left(\gamma_{k}^{ \pm}\right)$(бифуркация петли). Бифуркационные диаграммы систем сравнения изображены на общем рис. 3.12. Штриховкой показаны проекции поглощающих областей для различных классов траекторий.

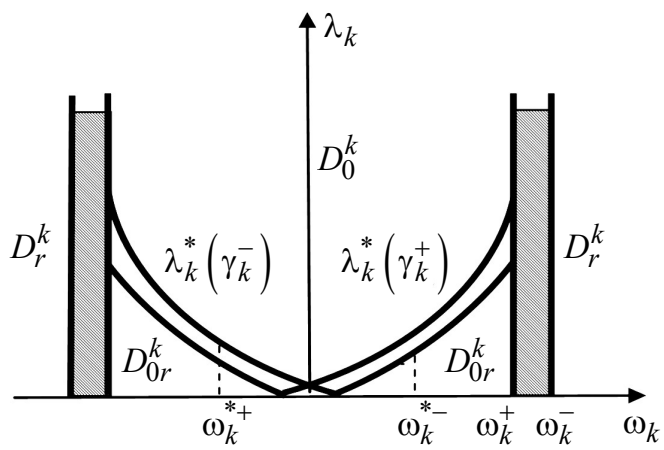

Рис. 3.12. Совмещенная бифуркационная диаграмма систем сравнения.

Очевидно, что для областей параметров $D_{(.)}^{k}=D_{(.) k}^{+} \cap D_{(.) k}^{-}$системы сравнения $A_{k}^{ \pm}$имеют одинаковые структуры траекторий на развертках $\left(\varphi_{k}, \dot{\varphi}_{k}\right)$. Из сказанного получаем следующие утверждения относительно областей параметров существования указанных выше классов предельных множеств траекторий системы (3.14).

Для области параметров $D_{00}=D_{0}^{1} \times D_{0}^{2}$ существует предельное множество траекторий $K_{00} \in G_{0}^{1} \times G_{0}^{2}$, реализующееся при любом 
начальном условии; для параметров $D_{0 r}=D_{0}^{1} \times D_{r}^{2}-$ глобально устойчивое множество $K_{0 r} \in G_{0}^{1} \times G_{r}^{2}$ : система из любого начального условия приходит в состояние, в котором первый ротатор испытывает колебательное движение, а второй вращается (о конкретном виде движений речь пока не идет); для параметров $D_{r 0}=D_{r}^{1} \times D_{0}^{2}-$ глобально устойчивое множество $K_{r 0} \in G_{r}^{1} \times G_{0}^{2}$; для области $D_{r r}=D_{r}^{1} \times D_{r}^{2}$ - глобально устойчивое множество $K_{r r} \in G_{r}^{1} \times G_{r}^{2}$. Вне данных областей параметров существуют совокупности множеств траекторий различных типов, реализующихся в зависимости от начальных условий. Картина взаиморасположения областей параметров представлена на рис. 3.13.

Введем другую форму системы связанных ротаторов, для чего рассмотрим физический пример [41]. На рис. 3.14 изображена электрическая схема двух сверхпроводящих переходов с емкостной связью.

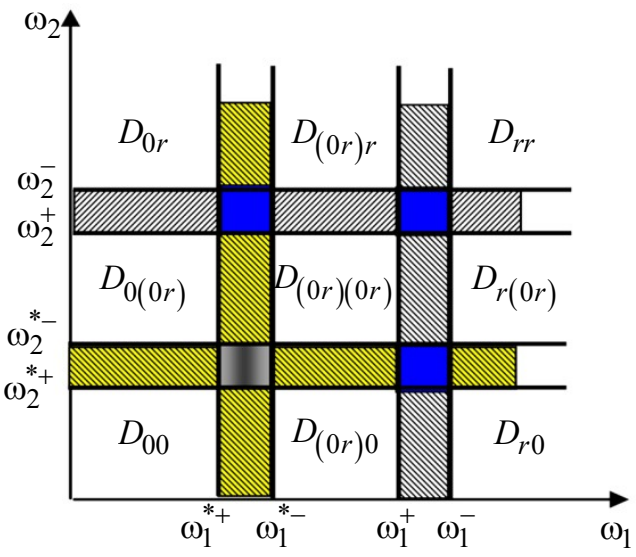

Рис. 3.13. Разбиение параметров на области различных классов траекторий.

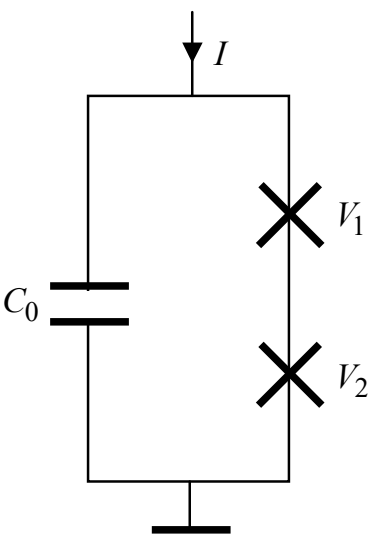

Рис. 3.14. Электрическая схема связанных сверхпроводящих переходов.

В физических переменных и параметрах правила Кирхгоффа и соотношение Джозефсона дают следующую динамическую модель системы: 


$$
\begin{aligned}
& C_{1} \frac{d V_{1}}{d t}+R_{1}^{-1} V_{1}+I_{c 1} \sin \varphi_{1}=I-C_{0} \frac{d\left(V_{1}+V_{2}\right)}{d t}, \\
& C_{2} \frac{d V_{2}}{d t}+R_{2}^{-1} V_{2}+I_{c 2} \sin \varphi_{2}=I-C_{0} \frac{d\left(V_{1}+V_{2}\right)}{d t}, \\
& \frac{d \varphi_{1}}{d t}=\frac{2 \pi}{\Phi_{0}} V_{1}, \frac{d \varphi_{2}}{d t}=\frac{2 \pi}{\Phi_{0}} V_{2} .
\end{aligned}
$$

Здесь $V_{1,2}$ - разности потенциалов сверхпроводников; $C_{1,2}, R_{1,2}$, $I_{c 1, c 2}$ - их емкости, нормальные сопротивления и значения критических сверхтоков соответственно; $C_{0}$ - емкость связи.

Введем физические и безразмерные параметры, а также безразмерное время следующим образом:

$$
\begin{aligned}
& I_{c}=\frac{I_{c 1}+I_{c 2}}{2}, R=\frac{R_{1}+R_{2}}{2}, \frac{2 \pi R I_{c}}{\Phi_{0}}=\Omega, \Omega t=\tau, C_{1,2} \Omega R=c_{1,2}, \\
& C_{0} \Omega R=c_{0}, r_{1,2}=\frac{R_{1,2}}{R}, v_{1,2}=\frac{I_{c 1, c 2}}{I_{c}}, i=\frac{I}{I_{c}} .
\end{aligned}
$$

При этом вместо (3.16) получаем безразмерную систему вида

$$
\begin{aligned}
& c_{1} \ddot{\varphi}_{1}+r_{1}^{-1} \dot{\varphi}_{1}+v_{1} \sin \varphi_{1}=i-c_{0}\left(\ddot{\varphi}_{1}+\ddot{\varphi}_{2}\right) \\
& c_{2} \ddot{\varphi}_{2}+r_{2}^{-1} \dot{\varphi}_{2}+v_{2} \sin \varphi_{2}=i-c_{0}\left(\ddot{\varphi}_{1}+\ddot{\varphi}_{2}\right) .
\end{aligned}
$$

После разрешения системы (3.17) относительно старших производных получаем модель (3.14). Связь параметров систем определяется формулами

$$
\begin{aligned}
& I=\frac{c_{1} c_{2}+c_{0}\left(c_{1}+c_{2}\right)}{c_{0}}, \delta_{1,2}=\left(1+\frac{c_{2,1}}{c_{0}}\right) \frac{1}{r_{1,2}}, \sigma_{1,2}=\left(1+\frac{c_{2,1}}{c_{0}}\right) v_{1,2}, \\
& \gamma_{1,2}=\frac{c_{2,1}}{c_{0}} i, a_{1,2}=\frac{1}{r_{2,1}}, b_{1,2}=v_{2,1}, \omega_{1,2}=r_{1,2} i .
\end{aligned}
$$

Далее наряду с системой (3.14) рассматриваем эквивалентную ей систему (3.17). Следующее утверждение исчерпывает вопрос о составе предельного множества траекторий $K_{00}$. 
В системе (3.17) и, соответственно, (3.14) отсутствуют ограниченные замкнутые фазовые траектории (предельные циклы колебательного типа). Это свойство устанавливается при помощи периодической функции Ляпунова

$$
V=\frac{1}{2} \sum_{k=1}^{2} c_{k} c_{0}^{-1} \dot{\varphi}_{k}^{2}+c_{0}^{-1} \sum_{k=1}^{2} \int_{\varphi_{0 k}}^{\varphi_{k}}\left(v_{k} \sin \varphi_{k}-i\right) d \varphi_{k}+\frac{1}{2}\left(\dot{\varphi}_{1}+\dot{\varphi}_{2}\right)^{2},
$$

производная которой вдоль векторного поля системы (3.17)

$$
\dot{V}=-\frac{1}{r_{1} c_{o}} \dot{\varphi}_{1}^{2}-\frac{1}{r_{2} c_{o}} \dot{\varphi}_{2}^{2},
$$

т. е. производная является неположительной во всем фазовом пространстве $G\left(\varphi_{1,2}, \dot{\varphi}_{1,2}\right)$. Это значит, что предельными траекториями множества $K_{00}$ являются состояния равновесия (и только они): устойчивое $O_{1}\left(\varphi_{1}, \varphi_{2}, \dot{\varphi}_{1}, \dot{\varphi}_{2}\right)=O_{1}\left(\arcsin \frac{i}{v_{1}}, \arcsin \frac{i}{v_{2}}, 0,0\right)$ (узел или фокус) и состояния равновесия седлового типа: $O_{2}\left(\pi-\arcsin \frac{i}{v_{1}}, \arcsin \frac{i}{v_{2}}, 0,0\right), \quad O_{3}\left(\arcsin \frac{i}{v_{1}}, \pi-\arcsin \frac{i}{v_{2}}, 0,0\right)$, $O_{4}\left(\pi-\arcsin \frac{i}{v_{1}}, \pi-\arcsin \frac{i}{v_{2}}, 0,0\right)$. Единственной бифуркацией состояний равновесия является слияние всей четверки, образование сложного состояния равновесия и его исчезновение при $i=\min \left(v_{1}, v_{2}\right)+0=i_{0}+0$.

2. Структуры траекторий и бифуркации вращательных движений ротаторов.

Рассмотрим качественную структуру множества вращательных движений $K_{r r}$. Параметры системы выбираем из тех областей, для которых существуют вращательные движения обоих ротаторов (см. рис. 3.13). Это может происходить при реализации начальных условий из части фазового пространства, если параметры выбира- 
ются из областей $D_{(0 r)(0 r)}, D_{(0 r) r}, D_{r(0 r)}$, или всего фазового пространства, если параметры выбираются из области $D_{r r}$.

Рассматриваем асимптотический случай $I \gg 1$. Заменой переменных (см. Приложение I)

$$
\begin{aligned}
& \dot{\varphi}_{1}=\omega_{1}+\mu \Phi_{1}\left(\varphi_{1}, \varphi_{2}, x_{1}\right), \dot{\varphi}_{2}=\omega_{2}+\mu \Phi_{2}\left(\varphi_{1}, \varphi_{2}, x_{2}\right), \\
& \Phi_{1}=\frac{\sigma_{1}}{\omega_{1}} \cos \varphi_{1}-\frac{b_{1}}{\omega_{2}} \cos \varphi_{2}+x_{1}, \Phi_{2}=\frac{\sigma_{2}}{\omega_{2}} \cos \varphi_{2}-\frac{b_{2}}{\omega_{1}} \cos \varphi_{1}+x_{2}, \\
& \mu=I^{-1}
\end{aligned}
$$

уравнения (3.14) сводятся к эквивалентной системе с быстровращающимися фазами $\varphi_{1,2}$. Эта система в зоне главного резонанса $\omega_{1} \approx \omega_{2}$ имеет усредненную систему вида

$$
\begin{aligned}
& \dot{x}_{1}=\mu\left(-\delta_{1} x_{1}+a_{1} x_{2}-B_{1} \sin \eta\right), \\
& \dot{x}_{2}=\mu\left(-\delta_{2} x_{2}+a_{2} x_{1}+B_{2} \sin \eta\right), \\
& \dot{\eta}=\mu\left(\Delta+x_{1}-x_{2}\right) .
\end{aligned}
$$

Здесь $\eta=\varphi_{1}-\varphi_{2}-$ фазовая расстройка, $\omega_{1}-\omega_{2}=\mu \Delta-$ частотная расстройка, $B_{1}=\frac{b_{1}\left(\sigma_{1}+b_{2}\right)}{2 \omega_{1} \omega_{2}}, B_{2}=\frac{b_{2}\left(\sigma_{2}+b_{1}\right)}{2 \omega_{1} \omega_{2}}$.

Заметим, что условие близости нормальных частот ротаторов $\omega_{1,2}$ означает, что, во-первых, параметры системы выбираются из малой окрестности диагонали плоскости $\left(\omega_{1}, \omega_{2}\right)$, а во-вторых, близость параметров $r_{1,2}: \mu \Delta=\left(r_{1}-r_{2}\right) i$.

В свою очередь, уравнения (3.18) при замене времени $\mu \Omega_{0} \tau=\tau_{n}, \quad \Omega_{0}=\left(\frac{B_{1}\left(\delta_{2}-a_{2}\right)+B_{2}\left(\delta_{1}-a_{1}\right)}{\delta_{1}+\delta_{2}}\right)^{\frac{1}{2}}$ сводятся к уже известному уравнению третьего порядка (см. раздел 2.1)

$$
\beta \dddot{\eta}+\ddot{\eta}+\alpha \cos \eta \dot{\eta}+\lambda^{r} \dot{\eta}+\sin \eta=\gamma^{r},
$$


где $\beta=\Omega_{0} /\left(\delta_{1}+\delta_{2}\right), \quad \alpha=\left(B_{1}+B_{2}\right) / \Omega_{0}\left(\delta_{1}+\delta_{2}\right), \quad \lambda^{r}=\frac{\delta_{1} \delta_{2}-a_{1} a_{2}}{\Omega_{0}\left(\delta_{1}+\delta_{2}\right)}$, $\gamma^{r}=\frac{\delta_{1} \delta_{2}-a_{1} a_{2}}{\Omega_{0}^{2}\left(\delta_{1}+\delta_{2}\right)} \Delta$. Качественные структуры траекторий этого уравнения известны (см. раздел 2.1 ), их интерпретация на систему (3.14) приведена выше.

Заметим, что в случае идентичных симметрично связанных ротаторов система (3.18) имеет единственное и ГАУ интегральное многообразие $x_{1}=-x_{2}$. Многообразие разбивается на траектории траекториями уравнения свободного маятника:

$$
\ddot{\eta}+(\delta+a) \dot{\eta}+2 B \sin \eta=0 .
$$

При переходе от (3.18) к (3.20) выполнено преобразование времени $\mu \tau=\tau_{n}$. В свою очередь, это уравнение имеет состояние равновесия $O(\eta, \dot{\eta})=O(0,0)$, которое ГАУ, т. е. динамика идентичных слабо нелинейных ротаторов в области параметров $D$ проста: при любом начальном условии с течением времени в связанной системе наблюдается синхронизация вращательных движений.

\section{3. Характеристики вращения ротаторов.}

Исследованием состояний равновесия системы (3.17) решен вопрос о нулевых ступенях характеристик вращения ротаторов, соответствующих сверхпроводящим ветвям вольтамперных характеристик (BAX) переходов в схеме на рис. 3.14. Нулевые ступени существуют и устойчивы в интервале $0 \leq i<i_{0}$.

Для описания резистивной части ВАХ переходов обратимся к системе (3.18) и уравнению (3.19). Уравнение (3.19) имеет два состояния равновесия: $O_{1}(\eta, \dot{\eta}, \ddot{\eta})=O_{1}\left(\arcsin \gamma^{r}, 0,0\right)$ (узел или фокус) и $O_{2}\left(\pi-\arcsin \gamma^{r}, 0,0\right)$ (седло). Состояние равновесия $O_{1}$ устойчиво во всей области существования (см. раздел 2.1) и соответствует предельному циклу системы (3.14) - режиму устойчивой взаимной синхронизации ротаторов. Область удержания (существования) синхронизации определяется системой неравенств: $\left|\gamma^{r}\right| \leq 1, i>i^{*}$. Первое из них соответствует существованию состоя- 
ния равновесия уравнения (3.19), а второе определяет область вращений ротаторов. $i^{*}-$ граница, отделяющая область вращений от области глобальной устойчивости состояния равновесия системы (3.17). Точное значение $i^{*}$ определяется экспериментально. Справедлива оценка $i^{*}=\max \left(r_{1}^{-1} \omega_{1}^{*_{-}}, r_{2}^{-1} \omega_{2}^{*_{-}}\right)$. В развернутом виде область существования синхронизации определяется двойным неравенством:

$$
i^{*}<i<i_{*}, i_{*}=\left(\frac{c_{0} v_{1} v_{2}}{\left|r_{1}-r_{2}\right|\left(c_{1}+c_{2}\right)\left(c_{1} c_{2}+c_{0} c_{1}+c_{0} c_{2}\right)}\right)^{\frac{1}{3}} .
$$

В режиме синхронизации $\Omega_{1}=\Omega_{2}=\frac{\omega_{1}+\omega_{2}}{2}=\frac{r_{1}+r_{2}}{2} i-$ среднее арифметическое парциальных частот, что вполне естественно.

Для получения полной картины ХВ к ветви синхронизации следует добавить ветви, соответствующие режимам биений ротаторов.

В силу ограниченности производных $\dot{\varphi}_{1,2}$ следует, что $\left\langle\ddot{\varphi}_{1}^{*}\right\rangle_{\tau}-\left\langle\ddot{\varphi}_{2}^{*}\right\rangle_{\tau}=\left\langle\ddot{\eta}^{*}\right\rangle_{\tau}=0$. В таком случае из замен переменных и системы (3.18) получаем

$$
\begin{gathered}
\Omega_{1,2}=\omega_{1,2}+d^{-1}\left(\left(\delta_{2,1}+a_{1,2}\right)\left(\left\langle\dot{\eta}^{*}\right\rangle_{\tau}-\mu \Delta\right)+\mu\left(B_{1}+B_{2}\right)\left\langle\sin \eta^{*}\right\rangle_{\tau}\right), \\
d=\delta_{2}-\delta_{1}+a_{1}-a_{2} .
\end{gathered}
$$

Из свойств уравнения (3.19): при увеличении параметра $\gamma^{r}$ (увеличении $\Delta$, а значит, параметра $i$ ) $\left\langle\dot{\eta}^{*}\right\rangle_{\tau} \rightarrow \mu \Delta,\left\langle\sin \eta^{*}\right\rangle_{\tau} \rightarrow 0$. Следовательно, $\Omega_{1,2} \rightarrow \omega_{1,2}$, т. е. прямые $i=r_{1,2}^{-1} \Omega_{1,2} \quad$ являются асимптотами для ветвей биений. Будем считать, что $r_{1}>r_{2}$, тогда $\gamma^{r}>0,\left\langle\dot{\eta}^{*}\right\rangle_{\tau}>0$, а следовательно, $\Omega_{1}-\Omega_{2}=\left\langle\dot{\eta}^{*}\right\rangle_{\tau}>0, \Omega_{1}>\Omega_{2}$. Ветви парциальных частот характеристик вращения, соответствующие биениям, располагаются по разные стороны ветви синхро- 
низации. Заметим также, что при изменении параметра $i$ параметры $\gamma^{r} \sim i^{3}$ и $\lambda^{r} \sim i$ изменяются вдоль кривой $\gamma^{r}=\alpha\left(\lambda^{r}\right)^{3}$, где $\alpha>0$.

На рис. 3.15 изображена одна из бифуркационных диаграмм в плоскости $\left(\lambda^{r}, \gamma^{r}\right)$ и кривые $L_{1,2,3}\left(\gamma^{r}=\alpha_{1,2,3}\left(\lambda^{r}\right)^{3}\right)$, соответствующие качественно различным случаям.

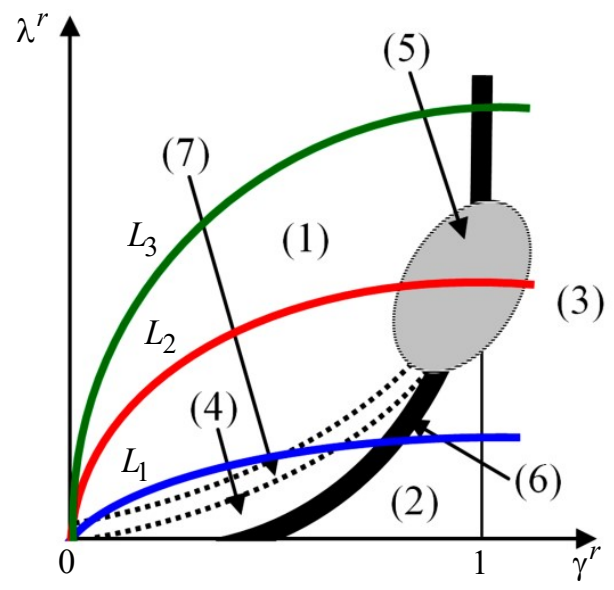

Рис. 3.15. Бифуркационная диаграмма параметров уравнения (3.19).

Напомним, что для параметров из области (1) в фазовом пространстве системы (3.14) существуют устойчивый (синхронизация) и неустойчивый предельные циклы, лежащие на резонансном торе $T^{2}$. Для области (4) в фазовом пространстве существуют резонансные циклы, а также устойчивый $T_{1}^{2}$ и неустойчивый $T_{2}^{2}$ двумерные торы (биения). Для области (2) существуют упомянутые циклы (не лежащие на торе, $T^{2}$ разрушается в области (6)), а также инвариантный тор $T_{1}^{2}$ (биения). Для параметров области (3) существует тор $T_{1}^{2}$, который является ГАУ, если параметры выбираются из области $D_{r r}$. Не исключено, что с изменением параметров на указанных торах могут рождаться и исчезать субгармонические резонансы, соответствующие кратной синхронизации [34, 42]. 
На рис. 3.16 представлены качественные картины характеристик вращения обоих ротаторов для различных параметров системы.

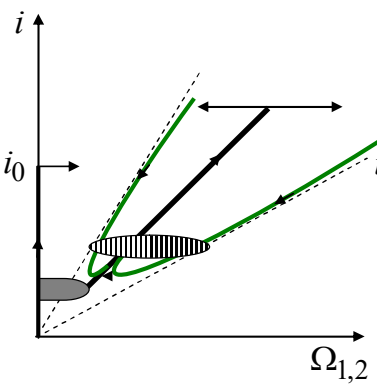

$\mathrm{a}$

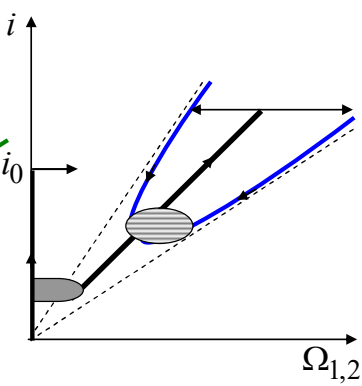

6

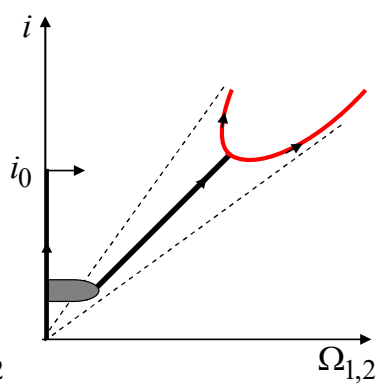

B

Рис. 3.16. Качественные картины характеристик вращения ротаторов.

А. Допустим что параметр $i$ квазистатически увеличивается от нуля и параметры $\lambda^{r}, \gamma^{r}$ изменяются (возрастают) вдоль кривой $L_{1}$. При этом ротаторы, находящиеся в состоянии равновесия при $i=0$, продолжают находиться в состоянии равновесия вплоть до его исчезновения при $i=i_{0}+0$. Эта ситуация определяет нулевую ступень ХВ того и другого ротаторов. При $i=i_{0}+0$ оба ротатора скачком переходят в режим вращений. В зависимости от начального условия может реализоваться режим простой периодической синхронизации или режим квазипериодических биений. В режиме синхронизации ХВ имеют общую ветвь, изображенную на рис. 3.16 отрезком прямой (первое приближение по малому параметру). Далее режим синхронизации, если таковой реализован, скачком (бифуркация слияния устойчивого и неустойчивого резонансных циклов и их исчезновение) сменяется режимом биений. Ветви XВ, соответствующие биениям, располагаются по разные стороны ветви синхронизации. Допустим теперь, что параметр $i$ уменьшается и движение параметров вдоль $L_{1}$ происходит из области (3) (см. рис. 3.15). При этом каждый из ротаторов находится в режиме устойчивых биений до тех пор, пока тор $T_{1}^{2}$ не исчезает от слияния с неустойчивым тором $T_{2}^{2}$ в области (7). В точке исчезновения $T_{1}^{2}$ ротаторы скачком возвращаются в режим синхрониза- 
ции. Верхняя заштрихованная область на рис. 3.16, а соответствует области (6) на рис. 3.15: здесь происходит разрушение резонансного тора, образование гомоклинических структур, связанных с гомоклинической кривой седлового резонансного цикла. Эта область на ХВ достаточно узка, как узка область $(6)(\sim \mu)$, а само хаотическое предельное множество не является аттрактором. Нижний заштрихованный участок на рис. 3.16, а относится к неизученной области параметров. Можно утверждать одно: переход ротаторов в состояние равновесия не может быть связан с любыми предельными множествами траекторий колебательного типа - регулярными или хаотическими.

Б. Данный случай (рис. 3.16,б) отличается от предыдущего тем, что возврат системы из режима биений в режим синхронизации происходит через хаотизацию вращений ротаторов. Как известно из раздела 2.1, область параметров (5) (см. рис. 3.15) соответствует хаотическим аттракторам, связанным с бифуркацией разрушения резонансного тора $T^{2}$ при переходе параметров из (1) в (5), бифуркацией разрушения тора $T_{1}^{2}$ при переходе параметров из области (3) в область (5), бифуркацией гомоклинической траектории седлового резонансного цикла, а также с бифуркациями удвоения периода двумерного тора. Хаотические аттракторы проиллюстрированы численным экспериментом в разделе 2.1. В заштрихованных областях на рис. 3.16,б имеет место эффект рассеяния ХВ ротаторов.

В. Этот случай (рис. 3.16,в) отличается от предыдущих мягким рождением режима квазипериодических биений при выходе системы из режима синхронизации: ветви биений на XВ примыкают к отрезку прямой, соответствующей синхронизации.

Изображенные на рис. 3.16 характеристики вращения ротаторов в случае системы с джозефсоновскими переходами (см. рис. 3.14) представляют их вольтамперные характеристики. 


\section{Глава 4 \\ КОЛЕБАНИЯ ВАЛОВ}

\section{1. Нелинейный резонанс изгибных колебаний гибкого ротора в системе с источником возбуждения ограниченной мощности}

Экспериментальное исследование и эксплуатация вращающихся валов (турбомашины различного назначения, насосы центробежного типа и др.) показывает, что в определенных ситуациях мощность их изгибных колебаний сравнима с мощностью исполнительного механизма. Это может происходить при разгоне вала до рабочей частоты вращения и прохождении его резонансных частот, а также при торможении механизма. Возможна ситуация, когда рабочая частота находится достаточно близко к одной из резонансных частот вала. Наконец, возможен дрейф резонансных частот к рабочей частоте вращения вследствие изменения колебательных свойств самого вала в процессе эксплуатации. В перечисленных случаях исследование динамики и расчет параметров валов не могут осуществляться без учета динамических свойств исполнительного механизма. Требуется исследование связанной системы гибкий ротор - исполнительный механизм.

Эффект «застревания» частоты вращения исполнительного механизма в окрестности резонансной частоты колебательной системы, известный как эффект Зоммерфельда [43-46], имеет место и в случае вращающихся валов. Этим эффектом и объясняется раскачка колебаний вала в резонансной зоне параметров, при которой значительная часть энергии источника переходит в энергию изгибных колебаний.

Задача исследования изгибных колебаний несбалансированных валов (гибких роторов) в системе с источником возбуждения ограниченной мощности является не новой, но не теряющей важности. Ее решению посвящены многочисленные работы расчетного характера. Аналитическое исследование, предложенное в $[47,48]$, не завершено по причине большой размерности системы. Ниже будет сделана попытка довести задачу до явного вида характеристик вращения исполнительного механизма и резонансных характеристик гибкого ротора. 
Рассматриваем однодисковую модель. Будем считать, что распределенная по длине масса вала, а также его оборудование приведены к диску. Этот диск расположен в середине невесомого и неподатливого на кручение вала. Эксцентриситет диска $e, W$ - геометрический центр, $S$ - центр тяжести, $O$ - положение равновесия диска (рис. 4.1).
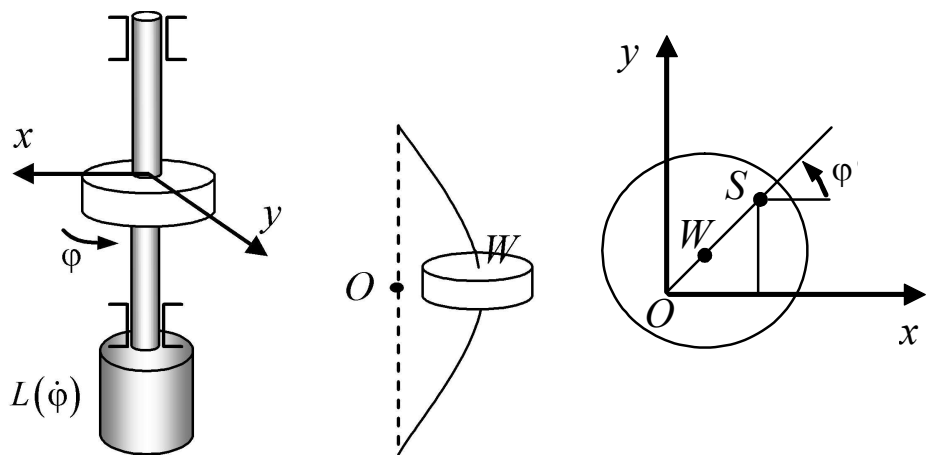

Рис. 4.1. Механическая модель системы гибкий ротор - двигатель.

В размерных переменных и параметрах динамика системы описывается уравнениями вида $[47,48]$

$$
\begin{aligned}
& m \ddot{x}+\varepsilon \dot{x}+c x+k(\dot{x}+\dot{\varphi} y)=c e \cos \varphi, \\
& m \ddot{y}+\varepsilon \dot{y}+c y+k(\dot{y}-\dot{\varphi} x)=c e \sin \varphi, \\
& I \ddot{\varphi}=L(\dot{\varphi})-q \dot{\varphi}-c e(x \sin \varphi-y \cos \varphi)-k(\dot{x} y-\dot{y} x)-k \dot{\varphi}\left(x^{2}+y^{2}\right) .
\end{aligned}
$$

Здесь $x, y$ - координаты центра тяжести диска в неподвижной системе координат, которая имеет начало на оси и перпендикулярна оси невозмущенного вала; $\varepsilon, k$ - коэффициенты внешнего и внутреннего затуханий при изгибных колебаниях; $c$ - жесткость вала в точке крепления диска; $m$ - масса диска; $I$ - момент инерции ротора двигателя; $\varphi$ - угол поворота; $L(\dot{\varphi})$ - движущий момент двигателя, включающий момент внутренних сил сопротивления движению ротора. Примем, что движущий момент асинхронного двигателя является линейной функцией вида $L(\dot{\varphi})=T-q \dot{\varphi}$, где $T-$ постоянная составляющая, а $q \dot{\varphi}-$ момент сил сопротивления дви- 
жению ротора [47]. В безразмерных переменных, параметрах и времени система (4.1) имеет вид

$$
\begin{aligned}
& \ddot{x}+\frac{\varepsilon \omega_{0}}{c} \dot{x}+x+\frac{k \omega_{0}}{c}(\dot{x}+\dot{\varphi} y)=\frac{e}{A_{0}} \cos \varphi, \\
& \ddot{y}+\frac{\varepsilon \omega_{0}}{c} \dot{y}+y+\frac{k \omega_{0}}{c}(\dot{y}-\dot{\varphi} x)=\frac{e}{A_{0}} \sin \varphi, \\
& \frac{I \omega_{0}^{2}}{T_{0}} \ddot{\varphi}=\frac{T}{T_{0}}-\frac{q \omega_{0}}{T_{0}} \dot{\varphi}-\frac{c e A_{0}}{T_{0}}(x \sin \varphi-y \cos \varphi)- \\
& -\frac{k \omega_{0} A_{0}^{2}}{T_{0}}\left(\dot{x} y-\dot{y} x+\dot{\varphi}\left(x^{2}+y^{2}\right)\right) .
\end{aligned}
$$

При переходе от (4.1) к (4.2) введено безразмерное время $\omega_{0} t=\tau, \omega_{0}^{2}=c / m$, а также выполнено масштабирование переменных $x, y$ постоянной $A_{0}$ и масштабирование моментов постоянной $T_{0}$. Величина масштабных постоянных не имеет принципиального значения. За безразмерными переменными сохранены прежние обозначения.

Рассмотрим область параметров

$$
\begin{aligned}
& c / m=\omega_{0}^{2}, q \omega_{0} / T_{0}=\gamma,\left(I \omega_{0}^{2} / T_{0}\right)^{-1}=\mu, \varepsilon \omega_{0} / c=\mu h, k \omega_{0} / c=\mu h_{1}, \\
& e / A_{0}=\mu v, c e A_{0} / T_{0}=\mu \lambda, k \omega_{0} A_{0}^{2} / T_{0}=\mu \chi,\left(T-q \omega_{0}\right) / T_{0}=\mu \Delta .
\end{aligned}
$$

В данном случае нет необходимости проводить «сложные» преобразования системы, подобные приведенным выше. Введем новую переменную в виде $\dot{\varphi}=1+\mu \xi$ (в новом времени частота осцилляторов равна единице), после чего система (4.2) приобретает вид

$$
\begin{aligned}
& \dot{x}=x_{1}, \\
& \dot{x}_{1}=-x+\mu F_{1}, \\
& \dot{y}=y_{1}, \\
& \dot{y}_{1}=-y+\mu F_{2}, \\
& \dot{\xi}=\mu F_{3}, \\
& \dot{\varphi}=1+\mu \xi,
\end{aligned}
$$


где

$$
\begin{aligned}
& F_{1}=v \cos \varphi-\left(h+h_{1}\right) x_{1}-h_{1} y, \\
& F_{2}=v \sin \varphi-\left(h+h_{1}\right) y_{1}+h_{1} x, \\
& F_{3}=\Delta-\gamma \xi-\lambda(x \sin \varphi-y \cos \varphi)-\chi\left(x_{1} y-y_{1} x\right)-\chi\left(x^{2}+y^{2}\right) .
\end{aligned}
$$

Величины порядка $\mu^{2}$ в системе (4.3) не выписаны как не оказывающие влияния на первое приближение усредненной системы, а значит, и на качественную динамику системы (4.1).

Система (4.3) представляет собой ротатор, нагруженный парой осцилляторов. Соотношение параметров $\left(T-q \omega_{0}\right) / T_{0}=\mu \Delta$ соответствует зоне главного резонанса: $\left(T-q \omega_{0}\right) / T_{0}=\left(T / q-\omega_{0}\right) q / T_{0}=$ $\left(\Omega_{0}-\omega_{0}\right) q / T_{0}=\mu \Delta$, т. е. вблизи частоты $\Omega_{0} \approx \omega_{0}$, где $\Omega_{0}-$ парциальная частота вращения двигателя.

Выполним в системе (4.3) замену переменных вида

$$
\begin{aligned}
& x=u_{1} \sin \varphi+v_{1} \cos \varphi, \quad x_{1}=u_{1} \cos \varphi-v_{1} \sin \varphi, \\
& y=u_{2} \sin \varphi+v_{2} \cos \varphi, \quad y_{1}=u_{2} \cos \varphi-v_{2} \sin \varphi .
\end{aligned}
$$

В результате получаем эквивалентную систему в стандартной форме:

$$
\begin{aligned}
& \dot{u}_{1}=\mu\left(v_{1} \xi+F_{1} \cos \varphi\right), \\
& \dot{v}_{1}=-\mu\left(u_{1} \xi+F_{1} \sin \varphi\right), \\
& \dot{u}_{2}=\mu\left(v_{2} \xi+F_{2} \cos \varphi\right), \\
& \dot{v}_{2}=-\mu\left(u_{2} \xi+F_{2} \sin \varphi\right), \\
& \dot{\xi}=\mu F_{3}, \\
& \dot{\varphi}=1+\mu \xi .
\end{aligned}
$$

Усредняя уравнения (4.4) по быстрой фазе, получаем систему вида

$$
\begin{aligned}
& \dot{u}_{1}=\mu\left(-\frac{h+h_{1}}{2} u_{1}-\frac{h_{1}}{2} v_{2}+v_{1} \xi+\frac{v}{2}\right), \\
& \dot{v}_{1}=\mu\left(-\frac{h+h_{1}}{2} v_{1}+\frac{h_{1}}{2} u_{2}-u_{1} \xi\right),
\end{aligned}
$$




$$
\begin{aligned}
& \dot{u}_{2}=\mu\left(-\frac{h+h_{1}}{2} u_{2}+\frac{h_{1}}{2} v_{1}+v_{2} \xi\right), \\
& \dot{v}_{2}=\mu\left(-\frac{h+h_{1}}{2} v_{2}-\frac{h_{1}}{2} u_{1}-u_{2} \xi-\frac{v}{2}\right), \\
& \dot{\xi}=\mu\left(\Delta-\gamma \xi-\frac{\lambda}{2}\left(u_{1}-v_{2}\right)-\frac{\chi}{2}\left[\left(u_{1}+v_{2}\right)^{2}+\left(v_{1}-u_{2}\right)^{2}\right]\right), \\
& \dot{\varphi}=1+\mu \xi .
\end{aligned}
$$

В системе за усредненными переменными сохранены прежние обозначения. Как видим, подсистема первых пяти уравнений системы отделена от шестого и может исследоваться отдельно. Эта подсистема имеет существенно упрощающее задачу свойство, которое сформулируем в виде следующего утверждения: в фазовом пространстве $G\left(u_{1}, u_{2}, v_{1}, v_{2}, \xi\right)$ системы (4.5) существует единственное и глобально асимптотически устойчивое инвариантное многообразие $M=\left\{u_{1}=-v_{2}, v_{1}=u_{2}\right\}$.

Докажем это утверждение. Введем в системе переменные вида $u_{1}+v_{2}=x_{n}, \quad v_{1}-u_{2}=y_{n}$. (При такой замене исследование свойств многообразия сводится к исследованию свойств состояния равновесия $u_{1}+v_{2}=x_{n}=0, v_{1}-u_{2}=y_{n}=0$. Далее индекс «n» опускаем.) Относительно этих переменных получаем систему

$$
\begin{aligned}
& \dot{x}=-\frac{h+2 h_{1}}{2} x+y \xi, \\
& \dot{y}=-\frac{h+2 h_{1}}{2} y-x \xi .
\end{aligned}
$$

Состояние равновесия $x=0, y=0$ системы (4.6) является единственным (очевидно). С другой стороны, производная функции Ляпунова $V=x^{2}+y^{2}$, вычисленная вдоль траекторий системы, имеет вид $\dot{V}=-\left(h+2 h_{1}\right)\left(x^{2}+y^{2}\right) \leq 0, \forall\left(u_{1}, u_{2}, v_{1}, v_{2}, \xi\right) \in G$, т. е. является отрицательной, что и доказывает утверждение.

По этому свойству все фазовые траектории системы (4.5) экспоненциально притягиваются гиперплоскостью $M=\left\{u_{1}=-v_{2}, v_{1}=u_{2}\right\}$ 
и остаются в сколь угодно малой ее окрестности при $\tau \rightarrow \infty$. Это позволяет заменить задачу исследования системы (4.5) исследованием системы на многообразии. Многообразие заполнено фазовыми траекториями трехмерной динамической системы вида

$$
\begin{aligned}
& \dot{u}=\mu\left(-\frac{h}{2} u+v \xi+\frac{v}{2}\right), \\
& \dot{v}=\mu\left(-\frac{h}{2} v-u \xi\right), \\
& \dot{\xi}=\mu(\Delta-\gamma \xi-\lambda u) .
\end{aligned}
$$

Динамика системы (4.5) полностью определяется динамикой системы (4.7).

Для ХВ ротатора имеем $\Omega=1+\mu\left\langle\xi^{*}\left(t, t_{0}\right)\right\rangle_{t}$, где $\xi^{*}\left(t, t_{0}\right)-$ решение, соответствующее траектории предельного множества системы, реализующейся при заданных начальных условиях. Будем изображать фрагмент $\mathrm{XB}$, соответствующий резонансной зоне, и поскольку $T \sim \Delta$ (связаны линейно), а $\Omega \sim\left\langle\xi^{*}(t)\right\rangle_{t}$ (связаны линейно), то изображать будем зависимость $\Delta=f\left(\left\langle\xi^{*}\right\rangle_{t}\right)$. Эта функция содержит все качественные особенности истинной ХВ и пересчитывается одна из другой.

Определение. Резонансной характеристикой (PX) гибкого ротора будем называть функцию $A(\Omega)=\max _{t} \sqrt{x_{*}^{2}+y_{*}^{2}}, \quad$ заданную над пространством параметров системы и пространством ее начальных условий, где $x_{*}=x_{*}\left(t, t_{0}\right), y_{*}=y_{*}\left(t, t_{0}\right)-$ финальное решение системы при соответствующем начальном условии.

Физически PX определяет максимальное отклонение гибкого ротора от невозмущенного положения в зависимости от частоты вращения двигателя. В частности, если двигатель имеет неограниченную мощность и его частота вращения не зависит от нагрузки, то РX представляет собой амплитудно-частотную характеристику колебательной системы. 
Так же, как и ХВ, будем изображать $\mathrm{PX}$ в резонансной зоне в виде $A=A(\Delta)$. Обе характеристики взаимосвязаны, поскольку $A=A(\Delta)-\mathrm{PX}$, а $\Delta=\Delta(\Omega)-\mathrm{XB}$. Геометрическая форма $\mathrm{PX}$ и устойчивость различных ее ветвей определяется формой и устойчивостью ХВ.

Свойства усредненной системы, свойства XВ и $P X$. Выполним в системе преобразование времени $\mu \tau=\tau_{n}$ и введем удобные обозначения: $\xi=x, u=y, v=z$. В результате вместо системы (4.7) получаем эквивалентную систему вида

$$
\begin{aligned}
& \dot{x}=-\gamma x-\lambda y+\Delta, \\
& \dot{y}=-\frac{h}{2} y+x z+\frac{v}{2}, \\
& \dot{z}=-\frac{h}{2} z-x y .
\end{aligned}
$$

Перечислим свойства системы (4.8).

1. Все решения системы ограничены. Это свойство устанавливается при помощи квадратичной формы $V=x^{2} / 2+y^{2} / 2+$ $+(z-\lambda)^{2} / 2$. Ее производная вдоль векторного поля системы $\dot{V}=-\gamma x^{2}-\frac{h}{2} y^{2}-\frac{h}{2} z^{2}+\Delta x+\frac{v}{2} y+\frac{\lambda h}{2} z$ является отрицательной вне некоторого шара $R^{2}$ (очевидно). Это значит, что в фазовом пространстве $G(x, y, z)$ существует шар $V \leq C^{2}$, поглощающий шар $R^{2}$, в который попадают и остаются в нем все фазовые траектории системы. Другими словами, $\|x\|,\|y\|,\|z\|<C<\infty$ при $t \rightarrow \infty$.

2. Система в зависимости от параметров имеет от одного до трех состояний равновесия, координаты которых являются решением уравнения

$$
f=\Delta=\gamma \omega+\frac{\lambda \nu h / 4}{\omega^{2}+h^{2} / 4} .
$$

Здесь $x_{0}=\omega, x_{0}-$ координата состояния равновесия. 
Заметим, что кривая $f(\omega)$ является частью $\mathrm{XB}$, соответствующей состояниям равновесия системы (4.8) (предельным циклам системы (4.1)). Соответствующая состояниям равновесия РХ имеет вид

$$
A(\Delta)=\frac{v / 2}{\sqrt{(\omega(\Delta))^{2}+h^{2} / 4}} .
$$

Нетрудно выяснить, что при выполнении неравенства $\frac{\gamma h^{2}}{\lambda v}<\frac{3 \sqrt{3}}{4}$ ХВ имеет два экстремума (три состояния равновесия для значений $\Delta$ между экстремумами), а при условии $\frac{\gamma h^{2}}{\lambda v}>\frac{3 \sqrt{3}}{4}$ она взаимно однозначна (одно состояние равновесия при всех $\Delta$ ).

3 . Если система при всех значениях параметра $\Delta$ имеет единственное состояние равновесия, то оно является ГАУ. Свойство устанавливается следующим образом.

Допустим, $O\left(x_{0}, y_{0}, z_{0}\right)$ - состояние равновесия системы. Выполним замену $x=x_{0}+u, y=y_{0}+v, z=z_{0}+w$, в результате которой система приобретает вид

$$
\begin{aligned}
& \dot{u}=-\gamma u-\lambda v, \\
& \dot{v}=-\frac{h}{2} v+x_{0} w+z_{0} u+u w, \\
& \dot{w}=-\frac{h}{2} w-x_{0} v-y_{0} u-u v .
\end{aligned}
$$

Производная функции Ляпунова $V=\frac{1}{2}\left(m u^{2}+v^{2}+w^{2}\right)$ вдоль траекторий системы (4.11) $\dot{V}=-\frac{h}{2}\left(\alpha_{1} u+v\right)^{2}-\frac{h}{2}\left(\alpha_{2} u+w\right)^{2} \leq 0$, $\forall(u, v, w) \in G$ является отрицательной во всем фазовом пространстве; $\quad \alpha_{1}=\left(m \lambda-z_{0}\right) / h, \quad \alpha_{2}=y_{0} / h, m-$ положительный корень уравнения $\lambda^{2} m^{2}-2\left(\lambda z_{0}+h \gamma\right) m+y_{0}^{2}+z_{0}^{2}=0$. Условие существования положительного корня совпадает с условием взаимной однознач- 
ности ХВ. Взаимно однозначным XВ, изображенным на рис. 4.2,a, соответствуют однозначные РХ на рис. 4.3,a.

4. Устойчивость состояний равновесия в случае невзаимно однозначных ХВ. Характеристическое уравнение системы в вариациях относительно произвольного состояния равновесия $O\left(x_{0}, y_{0}, z_{0}\right)$ имеет вид:

$$
p^{3}+a_{0} p^{2}+a_{1} p+a_{2}=0
$$

где $a_{0}=h+\gamma, a_{1}=h^{2} / 4+\omega^{2}-\frac{\lambda v}{2} \frac{\omega}{h^{2} / 4+\omega^{2}}+\gamma h, a_{2}=\gamma\left(h^{2} / 4+\omega^{2}\right)-$ $\frac{\lambda v h}{2} \frac{\omega}{h^{2} / 4+\omega^{2}}$. Условия устойчивости Гурвица выражаются неравенствами

$$
\begin{aligned}
& a_{1}=f_{1}(\omega)=h^{2} / 4+\omega^{2}+\gamma h-\frac{\lambda v}{2} \frac{\omega}{h^{2} / 4+\omega^{2}}>0, \\
& a_{2}=f_{2}(\omega)=\gamma\left(h^{2} / 4+\omega^{2}\right)-\frac{\lambda v h}{2} \frac{\omega}{h^{2} / 4+\omega^{2}}=\left(h^{2} / 4+\omega^{2}\right) \Delta_{\omega}^{\prime}>0, \\
& a_{0} a_{1}-a_{2}=f_{3}(\omega)=h\left(h^{2} / 4+\omega^{2}\right)+\gamma h(h+\gamma)-\frac{\lambda v \gamma}{2} \frac{\omega}{h^{2} / 4+\omega^{2}}>0 .
\end{aligned}
$$

Из второго неравенства (4.12) следует, что падающий участок ХВ является неустойчивым при любых параметрах: $\Delta_{\omega}^{\prime}<0 \Rightarrow a_{2}<0$. Каждая его точка соответствует седловому [39] состоянию равновесия. 
$h>\sqrt{\frac{3 \sqrt{3} \lambda v}{4 \gamma}}, \lambda=5, v=5, \gamma=1, h=8$

$$
\lambda=5, v=5, \gamma=1, h=1
$$

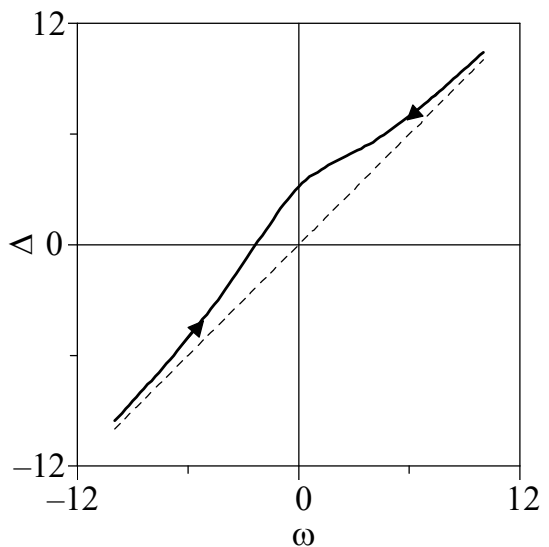

a

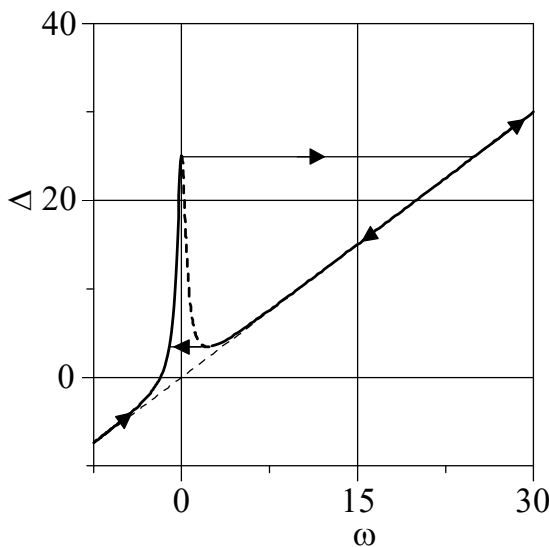

6

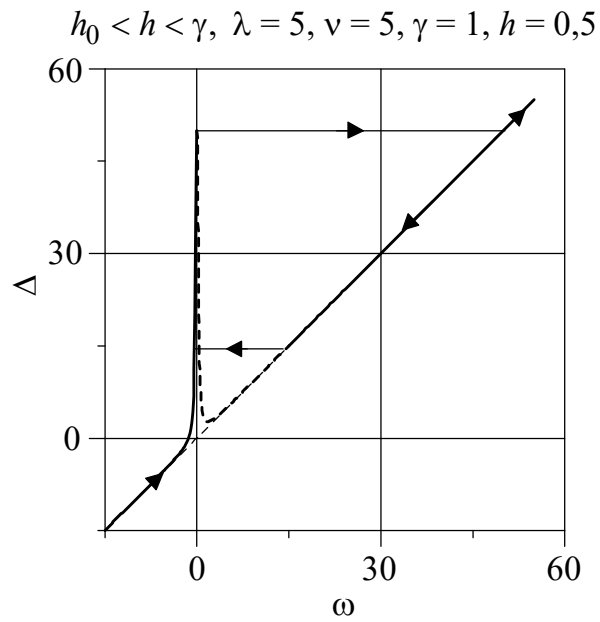

B

Рис. 4.2. Типы ХВ в резонансной зоне

5. При условии $h \geq \gamma$ оба возрастающих участка ХВ являются устойчивыми во всех своих точках. Это устанавливается следующим образом: поскольку $\Delta_{\omega}^{\prime}>0$, то $a_{2}>0$ и выполняется неравенство 


$$
\gamma\left(h^{2} / 4+\omega^{2}\right)>\frac{\lambda v h}{2} \frac{\omega}{h^{2} / 4+\omega^{2}} .
$$

В силу этого неравенства получаем:

$$
\begin{aligned}
& a_{1}=f_{1}(\omega)=h^{2} / 4+\omega^{2}+\gamma h-\frac{\lambda v}{2} \frac{\omega}{h^{2} / 4+\omega^{2}}>h^{2} / 4+\omega^{2}+ \\
& +\gamma h-\frac{\gamma}{h}\left(h^{2} / 4+\omega^{2}\right)=\frac{h-\gamma}{h}\left(h^{2} / 4+\omega^{2}\right)+\gamma h>0, \\
& a_{0} a_{1}-a_{2}=f_{3}(\omega)>h\left(h^{2} / 4+\omega^{2}\right)+\gamma h(h+\gamma)-\frac{\gamma^{2}}{h}\left(h^{2} / 4+\omega^{2}\right)= \\
& =\frac{h^{2}-\gamma^{2}}{h}\left(h^{2} / 4+\omega^{2}\right)+\gamma h(h+\gamma)>0 .
\end{aligned}
$$

Таким образом, в случае $h \geq \gamma$ условия устойчивости выполняются для всех точек возрастающих участков XВ, при этом состояния равновесия, соответствующие точкам возрастающих участков, являются устойчивыми фокусами или узлами. В экстремумах $\mathrm{XB}$ имеет место бифуркация слияния состояний равновесия с образованием седло-узла и последующим его исчезновением при соответствующем изменении параметра $\Delta$.

6. Устойчивость возрастающих участков ХВ в случае $h<\gamma$. Заметим, что в интервале $\omega<0$ условия устойчивости выполняются для всех точек ХВ. Заметим также, что условия устойчивости (первое и третье неравенства) усиливаются при увеличении $h$ $\left(\frac{\partial f_{1}}{\partial h}>0, \frac{\partial f_{3}}{\partial h}>0\right)$, что соответствует физическому смыслу этого параметра.

Допустим, что $h \ll 1$. В таком случае абсциссы левого (max) и правого ( $\mathrm{min})$ экстремумов XВ определяются соответствующими асимптотическими формулами:

$$
\omega_{1}=\frac{h^{3} \gamma}{8 \lambda v}, \quad \omega_{2}=\left(\frac{h \lambda v}{2 \gamma}\right)^{1 / 3} .
$$

Кроме того, на интервале $\omega<\omega_{1}$ имеют место асимптотические выражения вида 


$$
\begin{aligned}
& f_{1}=\gamma h-2 \lambda v \omega / h^{2}>0 \Rightarrow \omega<\gamma h^{3} / 2 \lambda \nu=4 \omega_{1}, \\
& f_{3}=\gamma^{2} h-2 \lambda v \gamma \omega / h^{2}>0 \Rightarrow \omega<\gamma h^{3} / 2 \lambda v=4 \omega_{1} .
\end{aligned}
$$

Таким образом, в данном случае условия устойчивости выполняются для всех точек левого возрастающего участка ХВ. В силу монотонного возрастания функций $f_{1}$ и $f_{3}$ по параметру $h$ эти условия выполняются при любых $h$ из интервала ограничений.

Для точек $\omega>\omega_{2}$ правого возрастающего участка получаем асимптотические формулы

$$
\begin{aligned}
& f_{1}=\omega^{2}-\lambda v / 2 \omega>0 \Rightarrow \omega>(\lambda v / 2)^{1 / 3}=\omega_{3}, \\
& f_{3}=\gamma^{2} h-\lambda v \gamma / 2 \omega>0 \Rightarrow \omega>\lambda v / 2 \gamma h=\omega_{4} .
\end{aligned}
$$

При изменении параметра $\omega$ от больших значений в сторону уменышения состояние равновесия на возрастающем участке теряет устойчивость, становится седло-фокусом и остается таковым на интервале $\omega_{3}<\omega<\omega_{4}$. Потеря устойчивости происходит от «влипания» в состояние равновесия неустойчивого предельного цикла, образующегося от петли сепаратрисы седлового состояния равновесия. При выходе параметра из данного интервала состояние равновесия становится седлом [39] и остается таковым на интервале $\omega_{2}<\omega<\omega_{3}$ вплоть до исчезновения в точке минимума ХВ. В силу монотонности $f_{1}$ и $f_{3}$ существует значение $h=h_{0}<\gamma$ такое, что на интервале $h<h_{0}$ часть правого участка ХВ является неустойчивой.

На основе исследованных свойств усредненной системы представим галерею качественно различных типов XВ и $\mathrm{PX}$ и опишем динамику системы при переходе через резонанс при различных значениях коэффициента диссипации. Считаем, что движущий момент изменяется квазистатически при постоянстве всех других параметров системы.

1) $h>\sqrt{\frac{3 \sqrt{3} \lambda \nu}{4 \gamma}}$. В этом случае ХВ являются взаимно однозначными. Пример такой ХВ приведен на рис. 4.2,a. РХ является однозначной функцией (рис. $4.3, a)$. При большой диссипации резонансные свойства вала проявляются слабо, прохождение резонанс- 
ной зоны, как в прямом, так и в обратном направлениях осуществляется по одному и тому же пути, без скачков частоты вращения двигателя.

2) $h_{0} \leq h<\sqrt{\frac{3 \sqrt{3} \lambda v}{4 \gamma}}$. Пример $\mathrm{XB}$ и соответствующей $\mathrm{PX}$ для параметров из этой области изображен на рис. 4.2,б и 4.3,б. В этом случае ХВ имеет два экстремума. Падающий участок ХВ неустойчив, а оба возрастающих участка устойчивы. При увеличении движущего момента двигателя - разгоне двигателя (стрелки вправо) происходит «застревание» частоты вращения двигателя по мере приближения к резонансной частоте вала (энергия, подводимая к двигателю, расходуется на раскачку колебаний вала). При достижении движущим моментом величины, соответствующей левому экстремуму XВ, происходит скачок частоты вращения двигателя в точку правой ветви, соответствующую устойчивому режиму вращений. При обратном изменении момента - торможении двигателя (стрелки влево) - скачок частоты двигателя происходит с левого экстремума ХВ в устойчивую точку правой ветви. Соответствующее движение по РХ показано стрелками на рис. 4.3,б.

3) $h<h_{0}$. Примеры ХВ и РХ для этого случая изображены на рис. 4.2,в и 4.3,в. Эффект «застревания» частоты вращения усиливается. Кроме того, при торможении двигателя скачок частоты вращения влево осуществляется не с минимума $\mathrm{XB}$, а из точки $\omega=\omega_{4}$ правой возрастающей ветви. Резонансная характеристика становится крайне узкой, так что на рисунке практически сливаются ее возрастающий и падающий участки.

Оценим скачок частоты двигателя и амплитуды изгибных колебаний в случае малых $h$. Скачок частоты вправо осуществляется с экстремума $\mathrm{XB}$, из точки $\omega \approx 0$. При этом $\Delta(0)=\lambda v / h$. Скачок осуществляется в точку XВ, практически лежащую на асимптоте $\Delta=\gamma \omega$, в точку $\omega=\frac{\lambda \nu}{\gamma h}$, т. е. $\delta \omega=\frac{\lambda \nu}{\gamma h}$. Из формул, связывающих размерные и безразмерные параметры, получаем $\delta \Omega=\frac{c^{2} e^{2}}{\varepsilon q \omega_{0}}$. При 
малых значениях параметра $\varepsilon$ величина скачка является существенной.

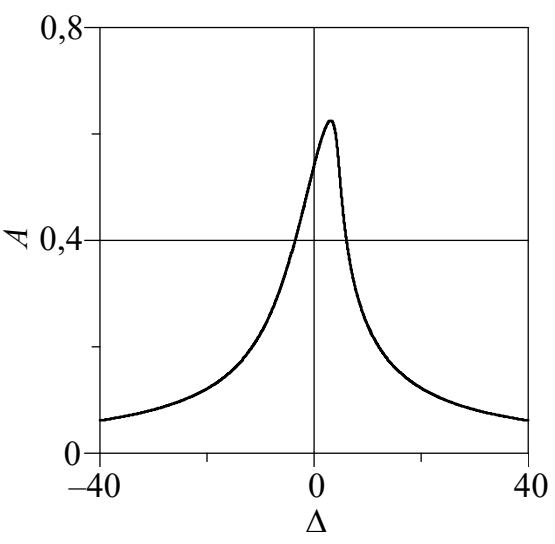

a

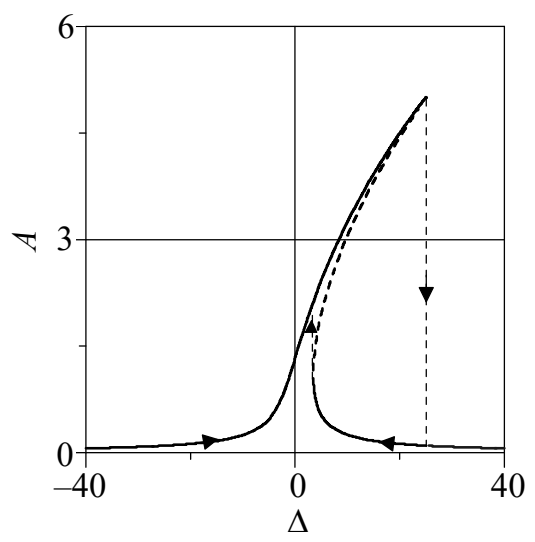

б

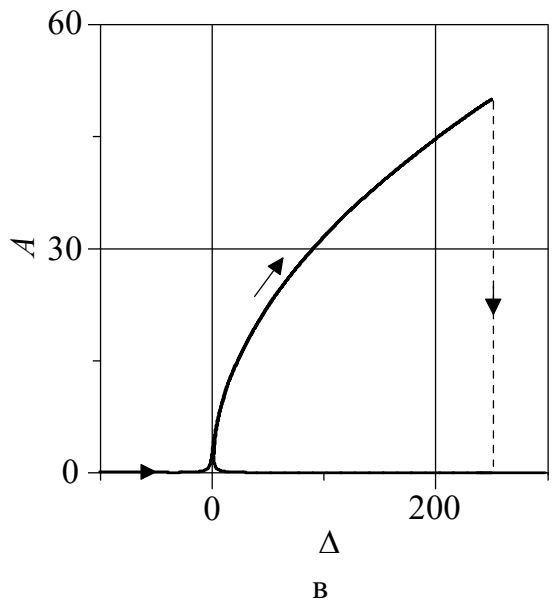

Рис. 4.3. Типы РХ в резонансной зоне: $\mathrm{a}-\lambda=5, v=5, \gamma=1, h=8$;

$$
\sigma-h=1 ; \text { в }-h=0,1
$$


Очевидно, что $\max A(\Delta)=A\left(\Delta_{0}\right)=\frac{v}{h}$ при $\Delta_{0}=\frac{\lambda v}{h}$. В физических параметрах $\max A(T / q)=A\left(\Omega_{0}\right)=\frac{e c}{\varepsilon \omega_{0}}$, при этом частота $\Omega_{0}=\omega_{0}+\frac{e^{2} c^{2}}{q \varepsilon \omega_{0}}$.

\section{2. Гашение изгибных колебаний вала модуляцией частоты вращения двигателя}

Расчет параметров изгибных колебаний валов показывает, что в условиях резонанса их амплитуда может достигать значительных и даже недопустимо больших значений. Как отмечалось, это может происходить как при разгоне вала до рабочей частоты вращения и прохождении резонансных зон, так и на рабочей частоте, если она близка к одной из резонансных частот. Эффект увеличения амплитуды колебаний на рабочей частоте может проявляться и вследствие изменения параметров вала в процессе эксплуатации, в частности при длительной эксплуатации погружных насосов, когда происходит солеотложение на лопастях крыльчаток. Это влечет за собой увеличение приведенной массы вала и смещение вниз спектра резонансных частот, а также рост его дисбаланса. Увеличению амплитуд изгибных колебаний способствует износ опорных подшипников (скольжения) вала в результате изменения параметров смазочного слоя, изменения их геометрии. Износ частей оборудования приводит к появлению существенных, а затем недопустимых вибраций, что в конечном итоге приводит к плачевному результату - выходу оборудования из строя.

Применение механических виброгасителей изгибных колебаний может оказаться неэффективным в силу двух причин: 1) недоступность системы в процессе эксплуатации, т. е. невозможность контроля и замены вышедших из строя устройств виброгашения; 2) невозможность изменения диапазона частот виброгасителей. Как уже говорилось, резонансные зоны могут смещаться в процессе эксплуатации. Ниже будет исследована возможность виброгашения изгибных колебаний путем управления частотой вращения испол- 
нительного механизма $[49,50]$, в частности асинхронного электродвигателя. Рассмотрим динамическую систему, описывающую изгибные колебания ротора, приводимого во вращение двигателем неограниченной (для простоты) мощности. Предположим, что при прохождении частоты вращения ротора через резонансную зону к движущему моменту двигателя подмешивается гармоническая составляющая. Гармоническая составляющая может подмешиваться и в рабочем состоянии системы, если контролирующие датчики будут указывать на недопустимый уровень вибраций. В указанных случаях постоянная частота вращения испытывает гармоническую модуляцию, а динамическая система имеет вид

$$
\begin{aligned}
& \ddot{x}+\frac{\varepsilon \omega_{0}}{c} \dot{x}+x+\frac{k \omega_{0}}{c}(\dot{x}+\dot{\varphi} y)=\frac{e}{A_{0}} \cos \varphi, \\
& \ddot{y}+\frac{\varepsilon \omega_{0}}{c} \dot{y}+y+\frac{k \omega_{0}}{c}(\dot{y}-\dot{\varphi} x)=\frac{e}{A_{0}} \sin \varphi, \\
& \dot{\varphi}=\Omega+A n \Omega \cos \left(n \Omega \tau+\psi_{0}\right) .
\end{aligned}
$$

Переменные, параметры и время в системе (4.13) безразмерны; $\Omega=\omega / \omega_{0}$, дифференцирование ведется по безразмерному времени $\omega_{0} t=\tau$.

Будем предполагать малыми коэффициенты внешнего и внутреннего затуханий, а также эксцентриситет ротора: $c / m=\omega_{0}^{2}$, $\varepsilon \omega_{0} / c=\mu h, k \omega_{0} / c=\mu h_{1}, e / A_{0}=\mu \nu$, где $\mu<<1-$ некоторый малый параметр. Система (4.13) приобретает следующий вид:

$$
\begin{aligned}
& \dot{x}=x_{1}, \\
& \dot{x}_{1}=-\Omega^{2} x+\mu F_{1}, \\
& \dot{y}=y_{1}, \\
& \dot{y}_{1}=-\Omega^{2} y+\mu F_{2}, \\
& \dot{\psi}=\Omega .
\end{aligned}
$$

Здесь $\quad F_{1}=v \cos \varphi-\left(h+h_{1}\right) x_{1}-h_{1} \dot{\varphi} y+\Delta x, \quad F_{2}=v \sin \varphi-\left(h+h_{1}\right) y_{1}+$ $+h_{1} \dot{\varphi} x+\Delta y, \varphi=\psi+A \sin \left(n \psi+\psi_{0}\right)$. 
Наша задача - ответить на вопрос: возможно ли, а если возможно, то каким образом следует задать параметры модуляции $A, n, \psi_{0}$, чтобы амплитуда изгибных колебаний была минимальной?

Физический взгляд на проблему может быть следующий. Система (4.13) и, соответственно, (4.14) - линейные неавтономные системы двух осцилляторов, каждый из которых является резонансным фильтром частоты $\Omega$ - первой гармоники в спектре внешнего воздействия:

$$
\begin{gathered}
\cos \varphi=\cos \left(\psi+A \sin \left(n \psi+\psi_{0}\right)\right), \sin \varphi=\sin \left(\psi+A \sin \left(n \psi+\psi_{0}\right)\right), \\
\psi=\Omega \tau .
\end{gathered}
$$

Эти функции представляются рядами Фурье с коэффициентами $J_{k}(A)$ - функциями Бесселя первого рода целочисленного аргумента. Естественно, что и решения уравнений также представляются подобными рядами (линейность модели). Если первая гармоника, имеющая наибольшую амплитуду в спектре колебаний осцилляторов, будет подавлена соответствующим выбором параметров модуляции, то амплитуды оставшихся гармоник будут иметь значения порядка величины воздействия, т. е. $\sim \mu<<1$. Это значит, что и максимальные отклонения вала от своей оси $x_{\max }, y_{\max }$ будут иметь тот же порядок величины $x_{\max }, y_{\max } \sim \mu(x(\tau), y(\tau)$ - многочастотные функции). Таким образом, наша задача - выбор параметров, обеспечивающих подавление первой гармоники.

Прямой путь к цели состоит в поиске решения уравнений (4.14) в виде рядов Фурье с неопределенными коэффициентами, определении этих коэффициентов и минимизации амплитуды первой гармоники. При этом отдельно должен решаться вопрос устойчивости. В целом такой подход к поставленной задаче сопряжен с громоздкими выкладками. По этой причине воспользуемся методом усреднения. При помощи замены

$$
\begin{array}{ll}
x=u_{1} \sin \psi+v_{1} \cos \psi, & x_{1}=\left(u_{1} \cos \psi-v_{1} \sin \psi\right) \Omega, \\
y=u_{2} \sin \psi+v_{2} \cos \psi, & y_{1}=\left(u_{2} \cos \psi-v_{2} \sin \psi\right) \Omega
\end{array}
$$

приводим систему (4.14) к стандартной форме вида 


$$
\begin{aligned}
& \dot{u}_{1}=\mu F_{1} \cos \psi, \\
& \dot{v}_{1}=-\mu F_{1} \sin \psi, \\
& \dot{u}_{2}=\mu F_{2} \cos \psi, \\
& \dot{v}_{2}=-\mu F_{2} \sin \psi, \\
& \dot{\psi}=\Omega .
\end{aligned}
$$

Перед процедурой усреднения заметим, что средние значения величин

$$
\begin{gathered}
\langle\dot{\varphi} y \sin \psi\rangle_{\psi},\langle\dot{\varphi} y \cos \psi\rangle_{\psi},\langle\dot{\varphi} x \sin \psi\rangle_{\psi},\langle\dot{\varphi} x \sin \psi\rangle_{\psi}, \\
\left\langle\cos \left(\psi+A \sin \left(n \psi+\psi_{0}\right)\right) \sin \psi\right\rangle_{\psi},\left\langle\cos \left(\psi+A \sin \left(n \psi+\psi_{0}\right)\right) \cos \psi\right\rangle_{\psi}, \\
\left\langle\sin \psi\left(\psi+A \sin \left(n \psi+\psi_{0}\right)\right) \sin \psi\right\rangle_{\psi},\left\langle\sin \left(\psi+A \sin \left(n \psi+\psi_{0}\right)\right) \cos \psi\right\rangle_{\psi}
\end{gathered}
$$

существенно зависят от параметра $n$, поэтому рассмотрим различные случаи.

1. Любые целые значения параметра $n>2$, а также любые иррациональные числа. Усредняя систему (4.15) по быстрой фазе $\psi$ и преобразуя время $\mu \tau=\tau_{n}$, получаем уравнения первого приближения по малому параметру вида

$$
\begin{aligned}
& \dot{u}_{1}=-\frac{h+h_{1}}{2} u_{1}-\frac{h_{1}}{2} v_{2}+\frac{\Delta}{2} v_{1}+\frac{1}{2} v J_{0}(A), \\
& \dot{v}_{1}=-\frac{h+h_{1}}{2} v_{1}+\frac{h_{1}}{2} u_{2}-\frac{\Delta}{2} u_{1}, \\
& \dot{u}_{2}=-\frac{h+h_{1}}{2} u_{2}+\frac{h_{1}}{2} v_{1}+\frac{\Delta}{2} v_{2}, \\
& \dot{v}_{2}=-\frac{h+h_{1}}{2} v_{2}-\frac{h_{1}}{2} u_{1}-\frac{\Delta}{2} u_{2}-\frac{1}{2} v J_{0}(A) .
\end{aligned}
$$

Здесь $J_{0}(A)$ - функция Бесселя первого рода. Заметим, что в рассматриваемом случае уравнения не зависят от начальной фазы $\psi_{0}$.

Рассматриваем величину $A^{*}=\sqrt{u_{10}^{2}+u_{20}^{2}+v_{10}^{2}+v_{20}^{2}}$, где $u_{10}$, $u_{20}, v_{10}, v_{20}$ - координаты состояния равновесия линейной систе- 
мы (4.16), которая является амплитудой первой гармоники. Как отмечалось выше, задача состоит в минимизации этой величины.

Как и в разделе 4.1, замечаем, что система (4.16) имеет инвариантное многообразие $M=\left\{u_{1}=-v_{2}, u_{2}=v_{1}\right\}$, которое является устойчивым. Факт доказывается аналогично: рассматриваем систему на многообразии $M=\left\{u_{1}=-v_{2}, u_{2}=v_{1}=v\right\}$

$$
\begin{aligned}
& \dot{u}=-\frac{h}{2} u+\frac{\Delta}{2} v+\frac{v}{2} J_{0}(A), \\
& \dot{v}=-\frac{h}{2} v-\frac{\Delta}{2} u .
\end{aligned}
$$

Значения координат состояния равновесия $\left(u_{0}, v_{0}\right)$ системы (4.17) пропорциональны $J_{0}(A)=0$, следовательно, амплитуда первой гармоники минимальна при минимальных значениях $\left|J_{0}(A)\right|$. В частности, она равна нулю (полное гашение) при всех значениях $A$, для которых $J_{0}(A)=0$. Как известно, функция Бесселя $J_{0}(A)$ имеет бесконечное число нулей (см. рис. 1.8). Например, первый нуль соответствует значению $A=2,4$, что соответствует минимальному значению $A$. Полагая теперь в системе (4.17) $A=2,4$, получаем состояние равновесия $u=0, v=0$, которое является устойчивым, что очевидно.

Таким образом, задавая параметры частотной модуляции: $A=2,4$, любое целое $n>2$ или любое иррациональное число, а также любое значение начальной фазы $\psi_{0}$ (например, $\left.\psi_{0}=0\right)$, получаем эффект полного гашения первой гармоники изгибных колебаний ротора. При этом амплитуда изгибных колебаний $\sim \mu<<1$.

Численный эксперимент. Поскольку внутреннее трение не влияет на амплитуду изгибных колебаний, то вместо системы (4.13) численно была исследована система вида

$$
\begin{aligned}
& m \ddot{x}+\varepsilon \dot{x}+c x=c e \cos \varphi, \\
& m \ddot{y}+\varepsilon \dot{y}+c y=c e \sin \varphi, \\
& \dot{\varphi}=\Omega+A n \Omega \cos (n \Omega t) .
\end{aligned}
$$


Эксперимент проводился для вала с параметрами $m=1, \varepsilon=0,1$, $c=25, e=1$ при различных параметрах системы гашения, т. е. параметрах модуляции. Интерес представляли два случая: 1) действие системы гашения, когда частота вращения двигателя фиксирована и находится в резонансе с частотой поперечных колебаний вала; 2) эффективность демпфирования поперечных колебаний вала в случае разгона двигателя с прохождением его частоты вращения через резонансную зону при постоянно действующей системе гашения.

На рис. 4.4,а представлена реализация решения $x=x^{*}(t)$ на резонансной частоте при отсутствии гашения (случай $n=0)$. В данном случае частота вращения двигателя постоянна и равна резонансной частоте поперечных колебаний вала. На рис. 4.4,б показано поперечное смещение вала $x(\omega(t))$ при разгоне ротора с прохождением через резонансную зону с выключенной системой гашения.

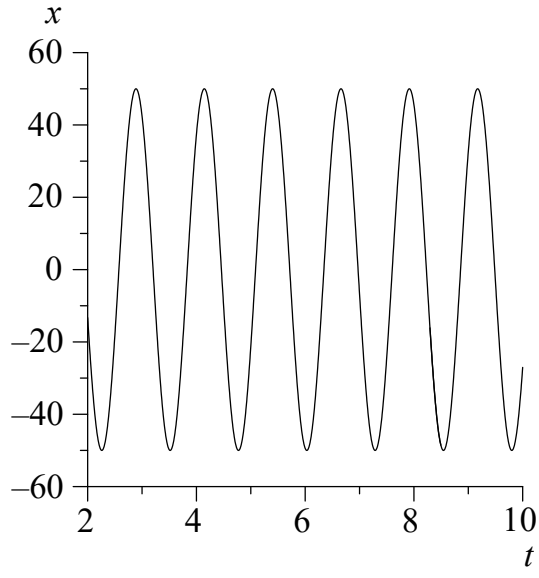

a

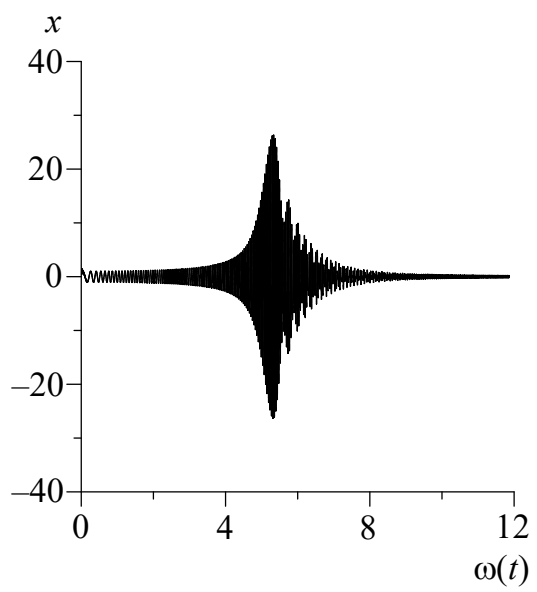

6

Рис. 4.4. Реализация решения $x=x^{*}(t)$ уравнений (4.13) при отсутствии демпфирования (a); поперечное смещение вала $x(\omega(t))$ при разгоне ротора и прохождении частоты его вращения через резонансную зону (б). 
Далее эффективность системы гашения будем оценивать коэффициентом гашения, равным отношению амплитуд поперечных колебаний вала без системы гашения (результаты взяты из рис. 4.4) и с включенной системой гашения. При этом рассматриваем два случая: первый - когда система гашения включается при фиксированной частоте вращения (резонансной), второй - при разгоне ротора и проходе его через резонансную зону с постоянно включенной системой гашения.

На рис. 4.5,a показан переходный процесс колебаний вала после включения системы гашения с параметрами $n=3, A=2,4$ (постоянная составляющая частоты вращения двигателя фиксирована и является резонансной). Рис. 4.5,б иллюстрирует установившиеся

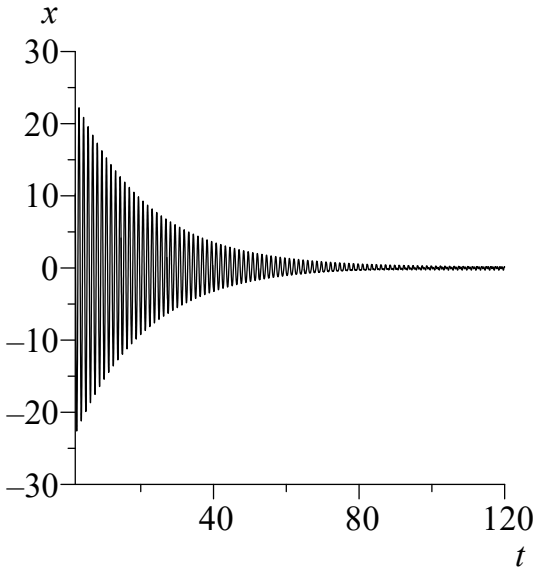

a

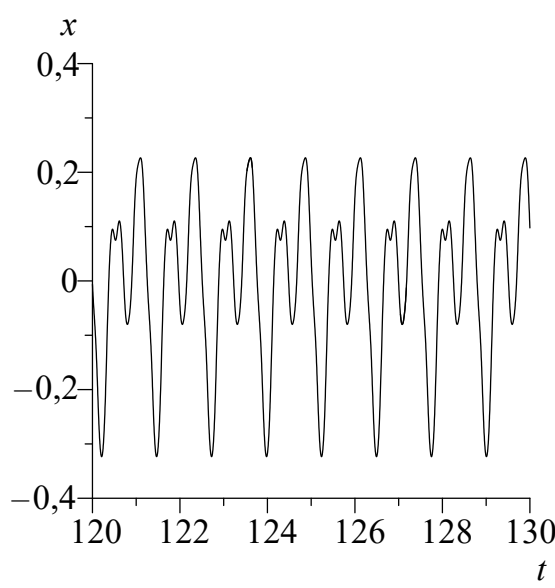

6

Рис. 4.5. Переходный процесс гашения колебаний (a); решение $x=x^{*}(t)$ как результат гашения изгибных колебаний (б); поперечное смещение вала $x(\omega(t))$ при разгоне ротора в резонансной зоне при постоянно включенной системе гашения (в); $n=3, A=2,4$ (окончание рис. см. на с. 128). 


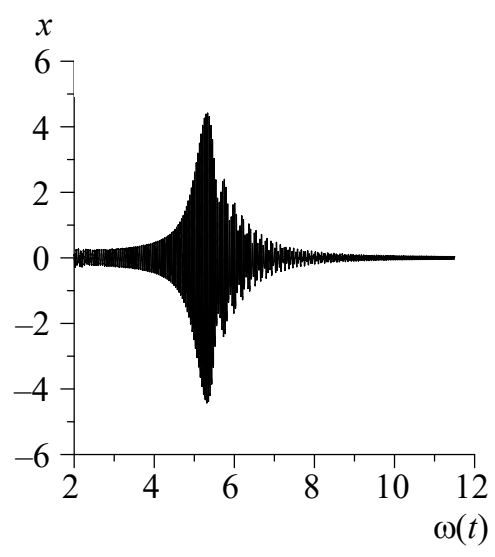

B

Рис. 4.5. Окончание.

колебания вала, как результат их демпфирования. На рис. 4.5,в изображено поперечное смещение вала $x(\omega(t))$ при разгоне ротора в резонансной зоне с постоянно включенной системой гашения. Вычисления показывают, что при фиксированной частоте вращения коэффициент гашения равен 217,4; при разгоне двигателя с постоянно действующей системой гашения коэффициент гашения равен 7,7. Существенная разница коэффициентов объясняется высокой добротностью колебательной системы и, соответственно, долгим процессом затухания собственных колебаний: система гашения не полностью справляется со своей работой за относительно малое время прохождения резонансной зоны.

2) Модулячия на частоте вращения ротора, $n=1$. В данном случае усредненная система имеет вид

$$
\begin{aligned}
& \dot{u}_{1}=-\frac{h+h_{1}}{2} u_{1}-\frac{h_{1}}{2} v_{2}+\frac{\Delta}{2} v_{1}+\frac{v}{2}\left(J_{0}+J_{2} \cos 2 \psi_{0}\right), \\
& \dot{v}_{1}=-\frac{h+h_{1}}{2} v_{1}+\frac{h_{1}}{2} u_{2}-\frac{\Delta}{2} u_{1}+\frac{v}{2} J_{2} \sin 2 \psi_{0}, \\
& \dot{u}_{2}=-\frac{h+h_{1}}{2} u_{2}+\frac{h_{1}}{2} v_{1}+\frac{\Delta}{2} v_{2}-\frac{v}{2} J_{2} \sin 2 \psi_{0}, \\
& \dot{v}_{2}=-\frac{h+h_{1}}{2} v_{2}-\frac{h_{1}}{2} u_{1}-\frac{\Delta}{2} u_{2}-\frac{v}{2}\left(J_{0}-J_{2} \cos 2 \psi_{0}\right) .
\end{aligned}
$$


Состояние равновесия системы (4.18) является устойчивым (соответствующая однородная система имеет устойчивое интегральное многообразие с заданной на этом многообразии устойчивой системой, см. выше). Это состояние равновесия будет иметь нулевые координаты вне зависимости от фазы $\psi_{0}$, если $J_{0}=J_{2}=0$ (начальная фаза является трудно контролируемым параметром). Как видно из рис. 1.8, корни этих бесселевых функций (начиная со второго корня, $A \approx 5,1$ ) являются достаточно близкими, поэтому, как и в предыдущем случае, соответствующим выбором амплитуды модуляции можно добиться практически полного подавления первой гармоники изгибных колебаний.

Численный эксперимент. На рис. 4.6 для параметров $n=1$, $A=5,1$ представлены демпфированные установившиеся колебания (рис. 4.6,a) и поперечное смещение вала $x(\omega(t))$ при разгоне ротора в резонансной зоне с включенной системой гашения (рис. 4.6,б). В первом случае коэффициент гашения равен 33,3, во втором - 7 .

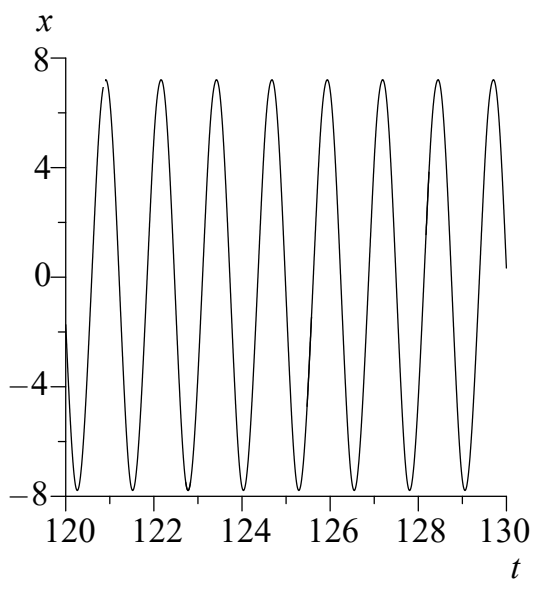

a $x$

6
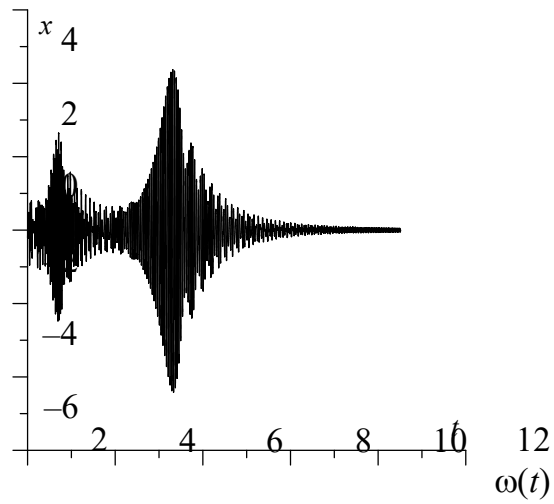

6

Рис. 4.6. Решение $x=x^{*}(t)$ как результат гашения изгибных колебаний (a); поперечное смещение вала $x(\omega(t))$ при разгоне ротора в резонансной зоне с постоянно включенной системой гашения (б); $n=1, A=5,1$ 
3) Модулячия на удвоенной частоте вращения ротора, $n=2$. В данном случае усредненная система имеет вид

$$
\begin{aligned}
& \dot{u}_{1}=-\frac{h+h_{1}}{2} u_{1}-\frac{h_{1}}{2} v_{2}+\frac{\Delta}{2} v_{1}-\frac{h_{1} A}{2}\left(-u_{2} \sin \psi_{0}+v_{2} \cos \psi_{0}\right)+ \\
& +\frac{v}{2}\left(J_{0}-J_{1} \cos \psi_{0}\right), \\
& \dot{v}_{1}=-\frac{h+h_{1}}{2} v_{1}+\frac{h_{1}}{2} u_{2}-\frac{\Delta}{2} u_{1}+\frac{h_{1} A}{2}\left(-u_{2} \cos \psi_{0}-v_{2} \sin \psi_{0}\right)- \\
& -\frac{v}{2} J_{1} \sin \psi_{0}, \\
& \dot{u}_{2}=-\frac{h+h_{1}}{2} u_{2}+\frac{h_{1}}{2} v_{1}+\frac{\Delta}{2} v_{2}+\frac{h_{1} A}{2}\left(-u_{1} \sin \psi_{0}+v_{1} \cos \psi_{0}\right)+ \\
& +\frac{v}{2} J_{1} \sin \psi_{0}, \\
& \dot{v}_{2}=-\frac{h+h_{1}}{2} v_{2}-\frac{h_{1}}{2} u_{1}-\frac{\Delta}{2} u_{2}-\frac{h_{1} A}{2}\left(-u_{1} \cos \psi_{0}-v_{1} \sin \psi_{0}\right)- \\
& -\frac{v}{2}\left(J_{0}+J_{1} \cos \psi_{0}\right) .
\end{aligned}
$$

При тех же рассуждениях, что и выше, получаем, что для полного подавления первой гармоники колебаний необходимо выполнение равенства $J_{0}=J_{1}=0$. Однако это уравнение не имеет решения, в том числе и приближенного, с удовлетворительной точностью (см. рис. 1.8). По этой причине естественным будет выбор значения амплитуды модуляции, при котором соответствующие функции имеют минимальное значение.

На рис. 4.7 показаны установившиеся демпфированные колебания (рис. $4.7, \mathrm{a})$ и поперечное смещение вала $x(\omega(t))$ при разгоне ротора и прохождении через резонансную зону при включенной системе гашения (рис. $4.7, б$ ) для случая $n=2, A=5,5$.

Вычисления показывают, что при включении системы гашения на резонансной частоте коэффициент гашения равен 2,85 ; при разгоне ротора в резонансной зоне с постоянно включенной системой гашения коэффициент гашения равен 33,3 . 


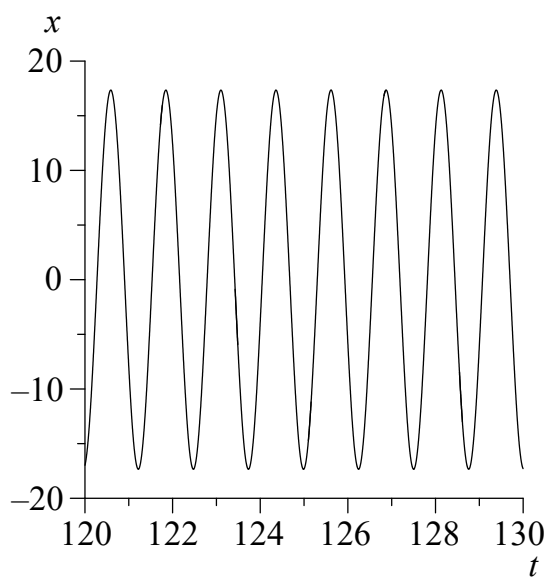

a

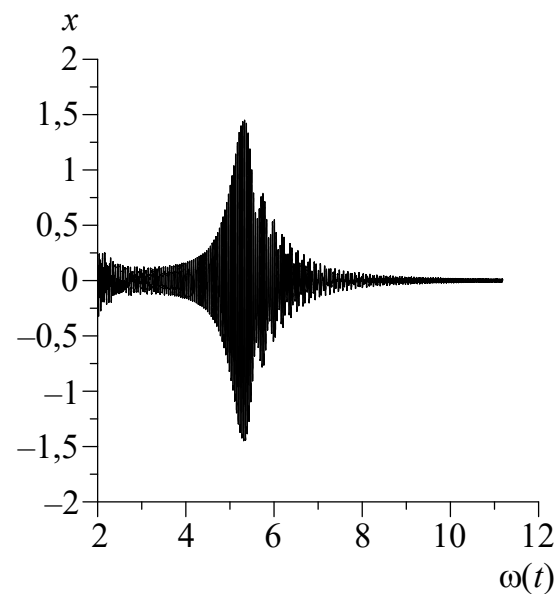

6

Рис. 4.7. Результат гашения изгибных колебаний при стационарном вращении ротора (а); поперечное смещение вала $x(\omega(t))$ при разгоне ротора в резонансной зоне при постоянно включенной системе гашения (б);

$$
n=2, A=5,5
$$

Как видим, принцип гашения изгибных колебаний вала путем модуляции частоты его вращения действует. Кроме того, этот метод допускает полную автоматизацию, что представляется важным, в частности, при эксплуатации глубоких нефтяных скважин.

\section{3. Хаотические крутильные колебания вала в системе с источником возбуждения ограниченной мощности}

В этом разделе мы рассмотрим динамику податливого на кручение (упругого) вала, приводимого в движение источником ограниченной мощности [51]. В качестве источника рассматриваем асинхронный электродвигатель, хотя таковым может быть исполнительный механизм и другого типа. Рассматриваем простую модель, когда упругий вал (система с распределенными параметрами) вместе с нагруженным на него оборудованием замещается одним диском с заданным моментом инерции. Диск расположен посередине вала (одномодовая модель). Будем предполагать, что все силы сопротив- 
ления линейны относительно угловых скоростей, а упругая восстанавливающая сила линейна относительно угла закрутки. Рассматриваемая физическая система схематично показана на рис. 4.8,a.

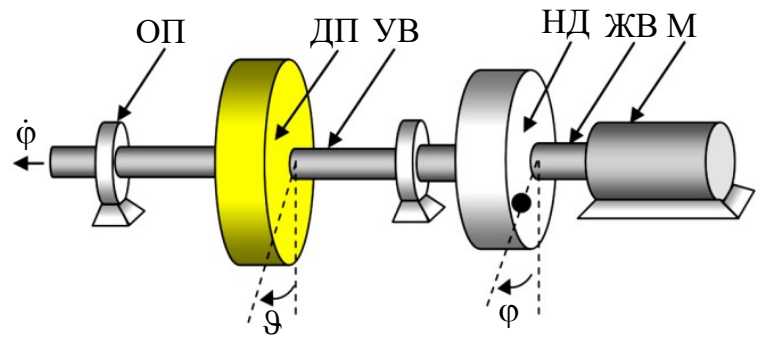

a

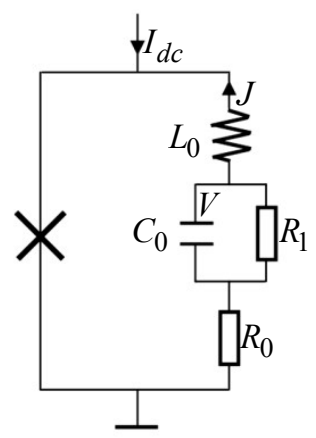

6

Рис. 4.8. Асинхронный мотор с несбалансированным диском и диском приведения упругого вала (а): М - асинхронный мотор, ЖВ - жесткий вал, НД - несбалансированный диск, УВ - упругий вал, ДП - диск приведения упругого вала, ОП - опорный подшипник; эквивалентная схема системы сверхпроводящий переход - резонатор (б).

Функция Лагранжа рассматриваемой системы имеет вид

$$
L=T+V=\frac{I_{1}}{2} \dot{\varphi}^{2}+\frac{I_{0}}{2} \dot{\vartheta}^{2}+m g e(1-\cos \varphi)+\frac{k}{2}(\varphi-\vartheta)^{2},
$$

где $\varphi, \vartheta$ - углы поворота несбалансированного диска и диска упругого вала; $I_{1}, I_{0}$ - моменты инерции дисков; $m$ - дисбаланс, $e$ - эксцентриситет, $k$ - крутильная жесткость упругого вала.

По условию движущий момент двигателя является линейной функцией скорости вращения $M_{d}=M_{0}-\lambda \dot{\varphi}$. Учтем, что вся система может находиться в вязкой среде (пример - погружные насосы центробежного типа). Считаем, что моменты всех диссипативных сил, действующих на несбалансированный диск, учтены членом $-\lambda \dot{\varphi}$ в выражении для движущего момента. Будем считать, что момент внешних сил сопротивления, действующих на диск приведения, выражен как $-\delta_{0} \dot{\vartheta}$. Допускаем также, что существует гипо- 
тетический момент сил крутильного внутреннего трения $\varepsilon_{0}(\dot{\varphi}-\dot{\theta})$. При сделанных предположениях получаем следующие уравнения движения системы:

$$
\begin{aligned}
& I_{1} \ddot{\varphi}+m g e \sin \varphi+k(\varphi-\vartheta)=M_{0}-\lambda \dot{\varphi}, \\
& I_{0} \ddot{\vartheta}+k(\vartheta-\varphi)=-\delta_{0} \dot{\vartheta}+\varepsilon_{0}(\dot{\varphi}-\dot{\vartheta}) .
\end{aligned}
$$

После введения новой переменной $\frac{k}{m g e}(\vartheta-\varphi)=J$ и времени $\frac{m g e}{\lambda} \tau=\tau_{n}$ модель принимает окончательный вид:

$$
\begin{aligned}
& I \ddot{\varphi}+\dot{\varphi}+\sin \varphi=\gamma+J, \\
& \ddot{J}+\delta \dot{J}+\omega_{0}^{2} J=d \dot{\varphi}+b \ddot{\varphi},
\end{aligned}
$$

где $I=\frac{I_{1} m g e}{\lambda^{2}}, \quad \gamma=\frac{M_{0}}{m g e}, \delta=\left(\delta_{0}+\varepsilon_{0}\right) I_{0}^{-1} \frac{\lambda}{m g e}, \omega_{0}^{2}=k I_{0}^{-1}\left(\frac{\lambda}{m g e}\right)^{2}$, $b=-\frac{k}{m g e}, d=-\frac{\delta_{0}}{\lambda} \omega_{0}^{2}$. Переменная $J$ имеет смысл угла закрутки вала.

Параллельно рассмотрим систему сверхпроводящий переход резонатор в одномодовом описании $[5,36]$. Эквивалентная электрическая схема этой системы изображена на рис. 4.8,б.

Законы Кирхгоффа и соотношение Джозефсона дают следующие уравнения для описания динамики системы (переменные, параметры и время безразмерны):

$$
\begin{aligned}
& c \ddot{\varphi}+\dot{\varphi}+\sin \varphi=\gamma+J, \\
& l_{0} \dot{J}+r_{0} J-V=-\dot{\varphi}, J=-c_{0} \dot{V}-r_{1}^{-1} V .
\end{aligned}
$$

Исключив переменную $V$, получим систему вида (4.20) с параметрами

$$
\delta=\frac{c_{0} r_{0} r_{1}+l_{0}}{c_{0} l_{0} r_{1}}, \omega_{0}^{2}=\frac{1}{c_{0} l_{0}}\left(1+\frac{r_{0}}{r_{1}}\right), b=-\frac{1}{l_{0}}, d=-\frac{1}{c_{0} l_{0} r_{1}} .
$$

Таким образом, для механической системы имеется квантовомеханический аналог и наоборот. 
Рассматриваем динамику системы (4.20) при условиях на параметры:

$$
I^{-1}=\mu \ll 1, \delta=\mu h, \gamma+\frac{d}{\omega_{0}}-\omega_{0}=\mu \Delta .
$$

При этих условиях система представляет собой квазилинейный ротатор, связанный с высокодобротным осциллятором. Последнее уравнение определяет резонансную зону. Модель (4.20) имеет внешнее сходство с (3.1). Однако, как будет видно, она обладает динамическими свойствами, совершенно отличными от предыдущей модели.

В целом порядок исследования рассматриваемой системы осуществляется в разработанной выше технике, варьируются только замены переменных, поэтому, не повторяя деталей, изложим кратко. Эквивалентная (4.20) система вида

$$
\begin{aligned}
& I \ddot{\varphi}+\dot{\varphi}+\sin \varphi=\gamma+J, \\
& \dot{J}=W+b \dot{\varphi}-\mu h J, \\
& \dot{W}=-\omega_{0}^{2} J+d \dot{\varphi}
\end{aligned}
$$

заменой переменных

$$
\begin{aligned}
& J=\theta \sin \varphi+\eta \cos \varphi+\frac{d}{\omega_{0}}, \\
& W=(\theta \cos \varphi-\eta \sin \varphi) \omega_{0}-b \omega_{0}, \\
& \dot{\varphi}=\omega_{0}+\mu \Phi(\theta, \eta, \varphi, \xi)
\end{aligned}
$$

сводится к эквивалентной системе в стандартной форме вида (3.4) с функциями

$$
\begin{aligned}
& \Theta=\left(\eta+b \sin \varphi+\frac{d}{\omega_{0}} \cos \varphi\right) \Phi-h(\theta \sin \varphi+\eta \cos \varphi) \sin \varphi+\frac{d}{\omega_{0}} \sin \varphi, \\
& T=\left(b \cos \varphi-\theta-\frac{d}{\omega_{0}} \sin \varphi\right) \Phi-h(\theta \sin \varphi+\eta \cos \varphi) \cos \varphi+\frac{d}{\omega_{0}} \cos \varphi, \\
& \Xi=\Delta-\left(\frac{\partial \Phi}{\partial \theta} \Theta+\frac{\partial \Phi}{\partial \eta} T+\frac{\partial \Phi}{\partial \varphi} \Phi+\Phi\right), \quad \Phi=\frac{1-\theta}{\omega_{0}} \cos \varphi+\frac{\eta}{\omega_{0}} \sin \varphi+\xi .
\end{aligned}
$$


Усредненная система имеет тот же вид, что и система (3.10):

$$
\begin{aligned}
& \dot{\xi}=\mu\left(-b_{1} \xi+b_{2} \theta+b_{3} \eta+\Delta\right), \\
& \dot{\theta}=\mu\left(-b_{4} \theta+b_{5} \eta+\eta \xi+b_{6}\right), \\
& \dot{\eta}=\mu\left(-b_{4} \eta-b_{5} \theta-\theta \xi+b_{7}\right), \\
& \dot{\varphi}=\omega_{0}+\mu \xi
\end{aligned}
$$

с параметрами

$$
\begin{gathered}
b_{1}=1-\frac{d}{\omega_{0}^{2}}, b_{2}=0, b_{3}=\frac{1}{2 \omega_{0}^{2}}, b_{4}=\frac{1}{2}\left(h+\frac{d}{\omega_{0}^{2}}\right), b_{5}=\frac{b}{2 \omega_{0}}, \\
b_{6}=\frac{d}{2 \omega_{0}^{2}}, b_{7}=\frac{b}{2 \omega_{0}} .
\end{gathered}
$$

В свою очередь, замена переменных

$$
\begin{aligned}
& x=b_{4}^{-1}\left(\xi+b_{5}\right), \quad y=\frac{b_{3} \eta+b_{2} \theta}{b_{1} b_{4}}-\Lambda, \\
& z=\frac{b_{3} \theta-b_{2} \eta}{b_{1} b_{4}}+R, \quad \mu b_{4} \tau=\tau_{n}
\end{aligned}
$$

сводит усредненную систему к системе лоренцевского типа

$$
\begin{aligned}
& \dot{x}=-\sigma(x-y)+\rho, \\
& \dot{y}=-y+R x-x z, \\
& \dot{z}=-z+x y+\Lambda x,
\end{aligned}
$$

параметры которой имеют выражения:

$$
\begin{gathered}
\sigma=b_{1} / b_{4}=\frac{2\left(\lambda+\delta_{0}\right)}{h \lambda-\delta_{0}}, \quad R=\frac{b_{2} b_{7}-b_{3} b_{6}}{b_{1} b_{4}^{2}}=\frac{\delta_{0} \lambda}{\omega_{0}^{2}\left(\lambda+\delta_{0}\right)\left(h \lambda-\delta_{0}\right)^{2}}, \\
\Lambda=\frac{b_{3} b_{7}+b_{2} b_{6}}{b_{1} b_{4}^{2}}=-\frac{k \lambda^{3}}{m g l \omega_{0}^{3}\left(\lambda+\delta_{0}\right)\left(\lambda h-\delta_{0}\right)^{2}} \rho= \\
=\left(\Delta+\left(b_{1} b_{4} b_{5}+b_{3} b_{7}+b_{2} b_{6}\right) b_{4}^{-1}\right) b_{4}^{-2} .
\end{gathered}
$$


На рассматриваемую систему переносятся все свойства из раздела 3.2 с учетом лишь того, что в нашем случае всегда $R>0$, а $\Lambda<0$.

Исходя из того, что усредненной системой для (4.20) является система (4.23), получаем утверждения об условиях существования регулярных и хаотических крутильных колебаний вала. В частности, утверждаем, что в системе существуют хаотические аттракторы Фейгенбаума и Лоренца, наблюдающиеся в пространстве точечного отображения Пуанкаре.

Численный эксперимент. На рис. 4.9, 4.10 представлены галереи фазовых портретов системы (4.20) на развертке фазового цилиндра, а также портретов точечного отображения. Точечное отображение $(x, \theta, \eta)_{\varphi=\varphi_{0}} \rightarrow(\bar{x}, \bar{\theta}, \bar{\eta})_{\varphi=\varphi_{0}+2 \pi}$ строилось для эквивалентной (4.20) системы вида

$$
\begin{aligned}
& \dot{\varphi}=\omega_{0}+\mu x, \\
& \dot{x}=-\mu x-\sin \varphi+\theta \sin \varphi+\eta \cos \varphi+\gamma-\omega_{0}, \\
& \dot{\theta}=\mu x \eta-\delta(\theta \sin \varphi+\eta \cos \varphi) \sin \varphi+\left(b \sin \varphi+\frac{d}{\omega_{0}} \cos \varphi\right)\left(\omega_{0}+\mu x\right), \\
& \dot{\eta}=-\mu x \theta-\delta(\theta \sin \varphi+\eta \cos \varphi) \cos \varphi+\left(b \cos \varphi-\frac{d}{\omega_{0}} \sin \varphi\right)\left(\omega_{0}+\mu x\right) .
\end{aligned}
$$

Эксперимент 1. Значения параметров: $d=-0,27508, \mu=0,01$, $\delta=0,0134, b=-0,005016, \omega_{0}=0,5$. На рис. 4.9 представлена галерея фазовых портретов и портретов точечных отображений Пуанкаре. Параметр системы $b=-0,005016$ выбран малым, что соответствует малому значению параметра $\Lambda$ усредненной системы. Резонансная зона при изменении параметра $\gamma$ проходит слева направо.

При $\gamma=1,0448906$ и меньше в системе (4.20) наблюдается периодическое движение - предельный цикл (рис. 4.9,a). На рис. 4.9,б показано установление периодического движения - неподвижной точки точечного отображения, которая является фокусом. 


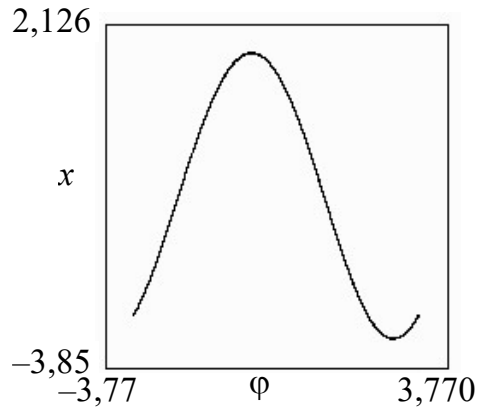

a

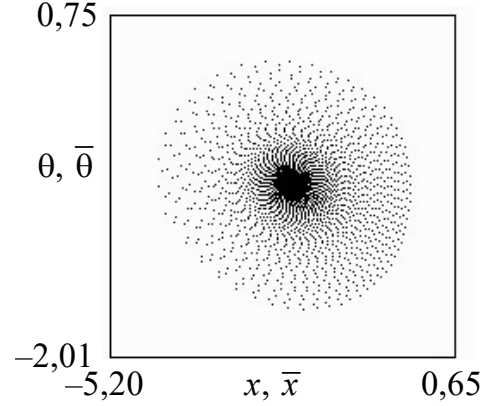

6

$$
\gamma=1,0448906
$$

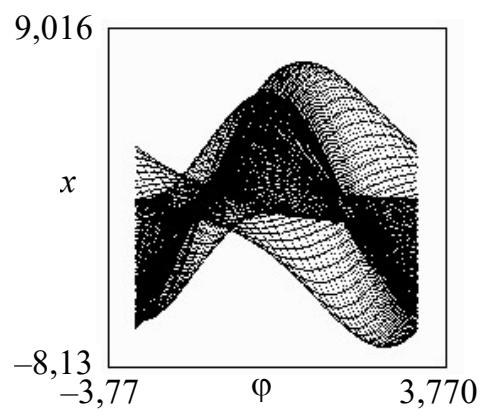

B

$$
\gamma=1,051491
$$

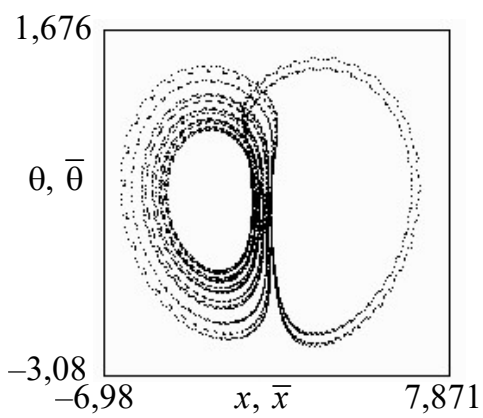

$\Gamma$

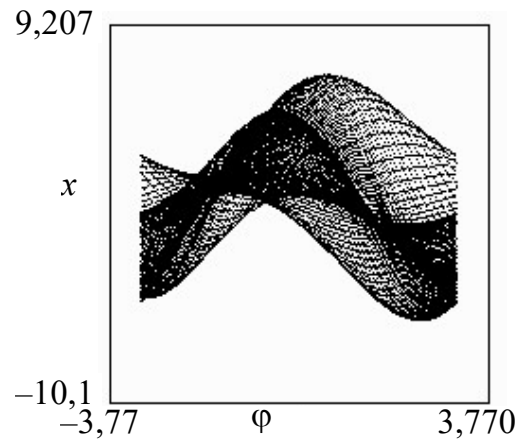

д

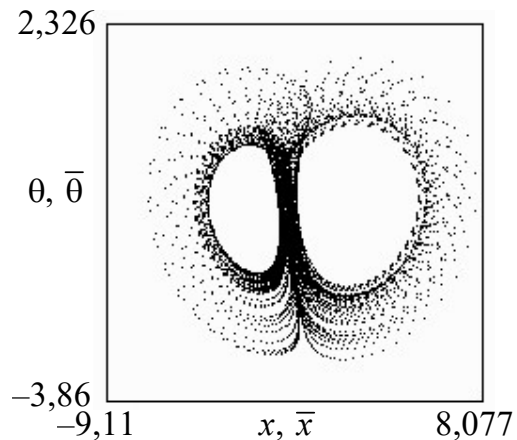

$\mathrm{e}$

$$
\gamma=1,058991
$$

Рис. 4.9. Галерея фазовых портретов и портретов точечного отображения в эксперименте 1 (окончание см. на с. 138). 


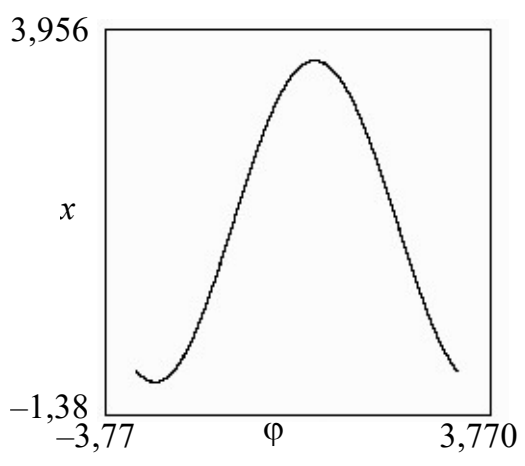

Ж

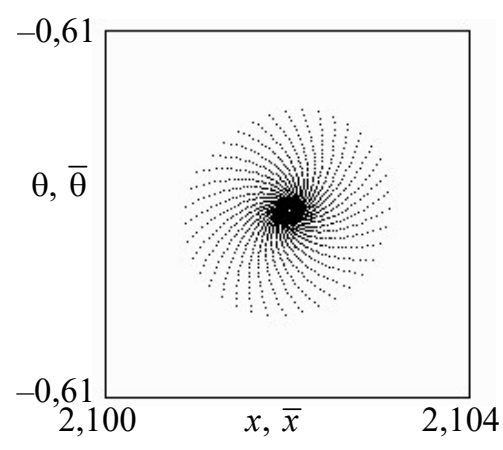

3

$$
\gamma=1,069291
$$

Рис. 4.9. Окончание.

При увеличении параметра $\gamma$ неподвижная точка теряет устойчивость через обратную бифуркацию Андронова - Хопфа - «влипания» в нее неустойчивого цикла (в фазовом пространстве усредненной системы и пространстве точечного отображения). При этом происходит «стягивание» к предельному циклу неустойчивого тора в четырехмерном фазовом пространстве системы (4.20) и «передача» циклу своей неустойчивости. В результате этой бифуркации система скачком из режима периодических колебаний переходит в режим хаотических колебаний, образом которых является асимметричный странный аттрактор Лоренца (рис. 4.9,в,г). При этом как мгновенная частота ротора двигателя, так и амплитуда крутильных колебаний начинают изменяться хаотически. Ситуация такова, что система, большее время находясь в окрестности левого предельного цикла, случайным образом переходит в окрестность правого цикла, а потом также случайным образом возвращается к прежнему циклу. Затем ситуация повторяется (период случаен). При переходе системы от цикла к циклу закрутка вала может достигать больших и недопустимо больших значений. Очевидно, что данный динамический режим является крайне неблагоприятным, если не катастрофическим, как для «выживаемости» вала, так и для самого исполнительного механизма.

При увеличении параметра $\gamma$ странный аттрактор сначала восстанавливает симметрию (рис. 4.9,д,е), а потом становится асимметричным вправо. Из правого предельного цикла рождается неус- 
тойчивый тор, а сам цикл становится устойчивым (рис. 4.9,ж,3). При увеличении $\gamma$ инвариантная кривая (в пространстве точечного отображения) «влипает» в петлю сепаратрисы седла, а неподвижная точка - предельный цикл - становится устойчивой глобально. Таким образом, при значениях параметров системы, соответствующих малым $\Lambda$, в системе имеется лоренцевский механизм рождения и исчезновения динамического хаоса.

Эксперимент 2. Значения параметров: $d=-0,27508, \mu=0,01$, $\delta=0,01252, b=-0,01875, \omega_{0}=0,5$. Заметим, что параметры усредненной системы очень «чувствительны» к изменению исходных параметров (см. формулы связи этих параметров), в частности параметра $b$. При заданных параметрах $\Lambda$ в системе (4.23) является существенным. В этом случае механизм рождения динамического хаоса иной. Галерея фазовых портретов и портретов точечных отображений представлена на рис. 4.10. Параметр $\gamma$ приближается к резонансной частоте слева. При $\gamma<1$ в системе имеется предельный цикл, который ГАУ. Далее цикл теряет устойчивость с рождением устойчивого тора (прямая бифуркация Андронова - Хопфа) (рис. 4.10,a). С увеличением $\gamma$ тор испытывает каскад бифуркаций удвоения одного из своих периодов (рис. 4.10,б-г) оставаясь всегда гладким и эргодическим (!). Цепочка удвоений заканчивается странным аттрактором Фейгенбаума (рис. 4.10,д,е). Далее наступает кризис - перемежаемость аттрактора Фейгенбаума с «остатками» несимметричного аттрактора Лоренца (рис. 4.10,ж,3). В этом случае изображающая точка большую часть времени проводит в окрестности аттрактора Фейгенбаума, совершая случайные выбросы в сторону правого состояния равновесия и немедленно возвращаясь назад. При этом реализация $J(t)$ представляет достаточно длинный цуг с хаотическим поведением амплитуды (для аттрактора Фейгенбаума степень хаотичности невелика) и редкими случайными всплесками большой амплитуды, прерывающими «хаотическую гармонию». Продолжение сценария развития и исчезновения динамического хаоса при дальнейшем увеличении параметра $\gamma$ происходит по той же схеме, что и в эксперименте 1. 


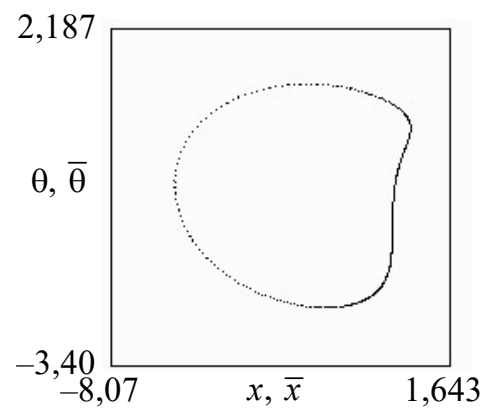

a

$$
\gamma=1,052491, \quad \gamma=1,055021
$$

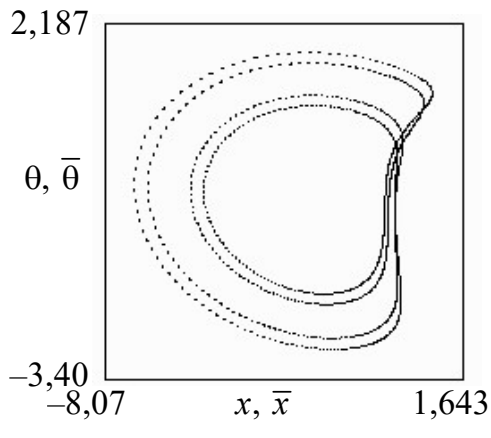

$$
\gamma=1,055288
$$

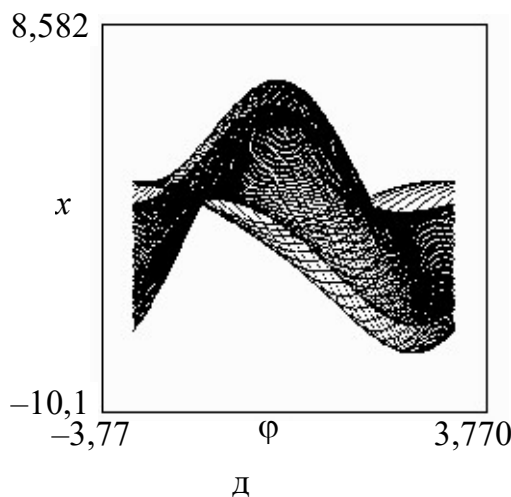

$$
\gamma=1,055795
$$

$\gamma=1,055487$

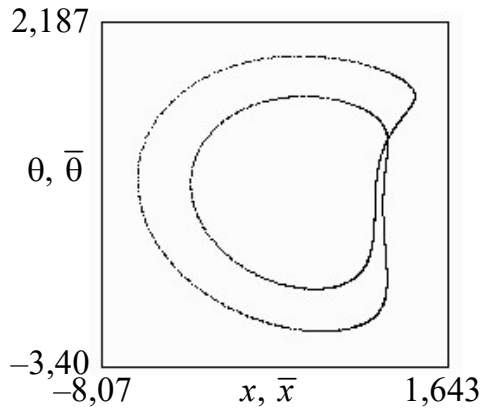

б
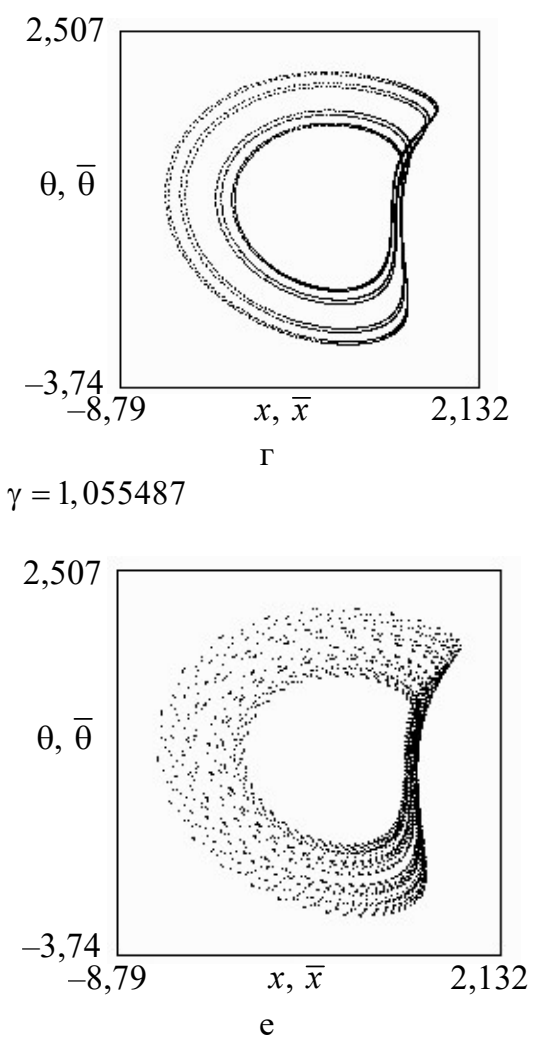

$\mathrm{e}$

Рис. 4.10. Галерея фазовых портретов и портретов точечного отображения в эксперименте 2 (окончание см. на с. 141). 


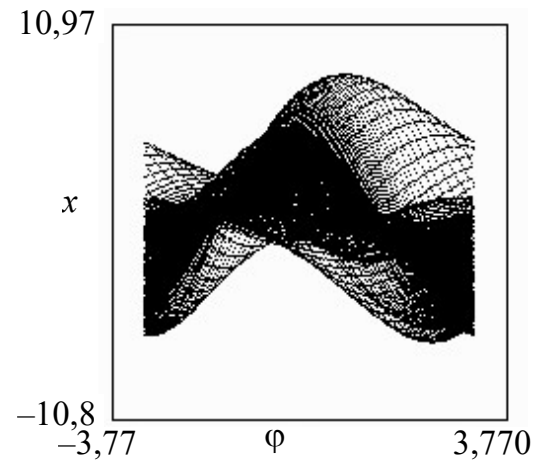

ж

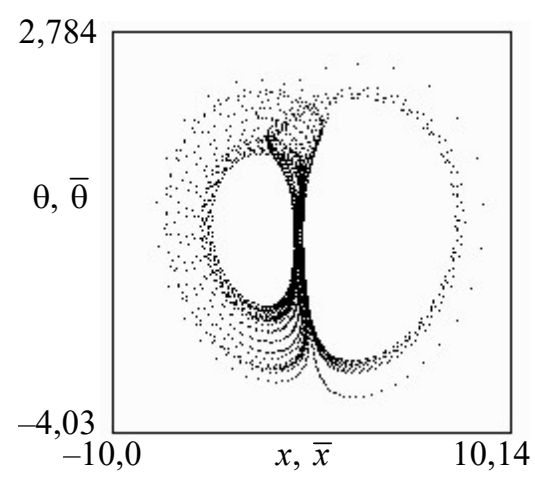

3

$$
\gamma=1,057391
$$

Рис. 4.10. Окончание.

Займемся качественными картинами характеристики вращения ротатора и резонансной характеристики крутильных колебаний вала. Для удобства перепишем выражения для состояний равновесия усредненной системы

$$
x_{0}=\omega, \quad y_{0}=\frac{R \omega-\Lambda \omega^{2}}{1+\omega^{2}}, \quad z_{0}=\frac{R \omega^{2}+\Lambda \omega}{1+\omega^{2}}
$$

и кривую состояний равновесия

$$
f=\omega+\frac{\Lambda \omega^{2}-R \omega}{1+\omega^{2}}, \quad f=\rho / \sigma .
$$

Характеристика вращения. Так же, как и в разделе 3.2, в качестве ХВ рассматриваем функцию $\rho=\rho\left(\overline{x^{*}\left(t, t_{0}\right)}\right)$, имеющую те же особенности, что и сама ХВ.

Резонансная характеристика (определение РХ дано в разделе 4.1). Выведем выражение для резонансной характеристики крутильных колебаний.

Из первого уравнения (4.21) получаем $J-\frac{d}{\omega_{0}}=$ $=\sqrt{\theta^{2}+\eta^{2}} \sin \left(\varphi+\varphi_{0}\right)$, т. е. выражение для амплитуды имеет вид 
$A=\sqrt{\theta^{2}+\eta^{2}}$. С другой стороны, из формул (4.22) получаем $(y+\Lambda)^{2}+(z-R)^{2}=\frac{b_{1}^{2} b_{4}^{2}}{b_{3}^{2}+b_{2}^{2}}\left(\theta^{2}+\eta^{2}\right), \quad$ т. e. $\quad A=\frac{\sqrt{b_{3}^{2}+b_{2}^{2}}}{b_{1} b_{4}} \times$ $\times \sqrt{\left(y^{*}+\Lambda\right)^{2}+\left(z^{*}-R\right)^{2}}$, где $y^{*}\left(t, t_{0}\right), z^{*}\left(t, t_{0}\right)$ - стационарное решение усредненной системы (4.23), реализующееся при заданном начальном условии.

Для состояний равновесия (4.24) (предельных циклов исходной системы (4.20)) получаем $\left(y^{*}+\Lambda\right)^{2}+\left(z^{*}-R\right)^{2}=\frac{R^{2}+\Lambda^{2}}{1+\omega^{2}}, \quad$ т. е. $A=\frac{\sqrt{b_{3}^{2}+b_{2}^{2}} \sqrt{R^{2}+\Lambda^{2}}}{b_{1} b_{4}} \frac{1}{\sqrt{1+\omega^{2}}}$. Поскольку нас интересует качественное поведение PX, то далее будем рассматривать ее в нормированном виде:

$$
A^{*}(\rho)=\frac{1}{\sqrt{1+(\omega(\rho))^{2}}} .
$$

Уравнения (4.25) и (4.26) представляют PX в параметрической форме (параметром является $\omega)$ ).

На рис. 4.11-4.13 представлены галереи ХВ и соответствующих РХ для различных значений параметров $\Lambda$ и $R$.

На рис. $4.11 \Lambda=0$. Хотя это и нефизичный случай, однако соответствующим образом будут вести себя означенные характеристики при малых значениях параметра $\Lambda$ (численный эксперимент 1). Как всегда, устойчивые участки изображены сплошными линиями, неустойчивые - пунктирными.

При $R<1 \mathrm{XВ}$ являются взаимно однозначными и система вал двигатель ведет себя подобно линейной системе: РХ является однозначной функцией (рис. 4.11,а,б).

При $R>1$ ХВ имеет гистерезисную петлю. При этом скачки частоты ротатора (вправо при разгоне двигателя и влево при его торможении) осуществляются с экстремумов XВ, а скачки ампли- 
туды крутильных колебаний осуществляются всегда вниз из точек РХ с вертикальной касательной $\left(\frac{d \rho}{d A}=0\right)$.

По мере возрастания параметра $R$ нарушаются условия устойчивости состояний равновесия для точек возрастающих участков XВ (обратная бифуркация Андронова-Хопфа), что приводит к уменьшению глубины гистерезиса - «обрезанию» петли. То же самое происходит и с РX. На рис. 4.11,в,г изображены примеры соответствующих ХВ и РХ. Стрелками указано движение по гистерезисным петлям. Динамика системы является регулярной во всем диапазоне изменения параметра $\rho$.

По мере приближения параметра $R$ к значению $R_{c}$ (см. раздел 3.2) глубина гистерезиса $\mathrm{XB}$, соответствующей состояниям равновесия, стремится к нулю, а РХ становится безгистерезисной. Однако это не полная картина ХВ и РХ. В этом случае, кроме состояний равновесия системы (4.23) (предельных циклов системы (4.20)), в ее фазовом пространстве уже существует странный аттрактор Лоренца, родившийся ранее (он существует при $\rho=0$, а значит, и при малых значениях $|\rho|$ ). То есть при одних начальных условиях может реализовываться безгистерезисная PX, а при других начальных условиях движения ротора двигателя, как и крутильные колебания вала, становятся хаотическими. При $R=R_{c}+0$ и $\rho=0$ аттрактор Лоренца становится единственным аттрактором системы (4.23), т. е. при медленном разгоне двигателя скачок частоты его вращения происходит не в устойчивое состояние, как ранее, а на странный аттрактор. При этом хаотическое изменение частоты вращения происходит в достаточно большом интервале. То же самое происходит с амплитудой крутильных колебаний. По всей видимости, этот режим является крайне неблагоприятным как для самого двигателя, так и для вала. Зона хаотических колебаний (по параметру $\rho$ ), возникающая при значении $R=R_{c}$, расширяется по мере возрастания $R$.

На рис. 4.11,д,е представлены ХВ и РХ в случае существования странных аттракторов в качестве единственных аттракторов в значительном интервале значений параметра $\rho$. При разгоне двигателя прохождение зоны хаотических колебаний происходит неотврати- 
мым образом; то же самое - при торможении двигателя. В заштрихованных областях имеет место эффект рассеяния ХВ и $\mathrm{PX}$.
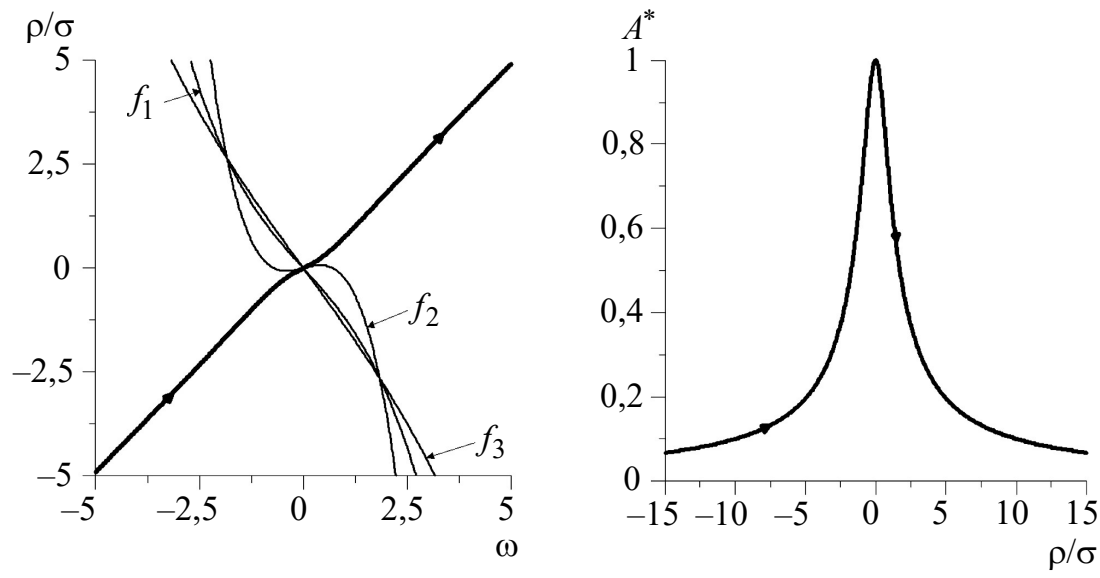

$$
\lambda=0, R=0,5
$$

$\mathrm{a}$
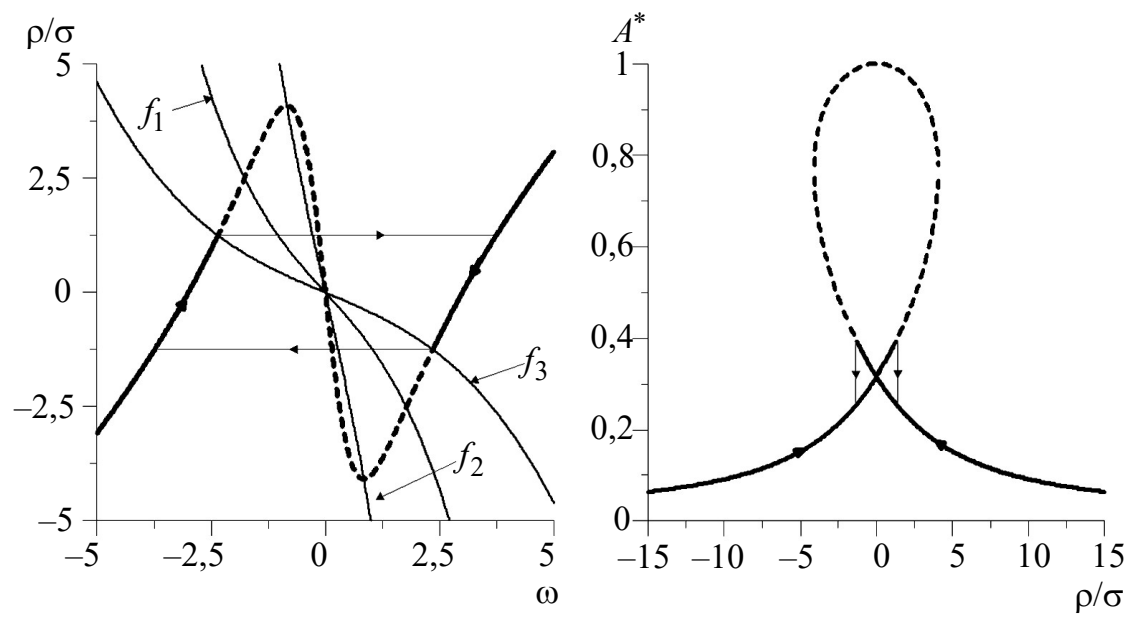

$$
\lambda=0, \quad R=10
$$

B

$\Gamma$

Рис. 4.11. ХВ и РХ при $\lambda=0$ и различных значениях параметра $R$ (окончание рис. см. на с. 145). 


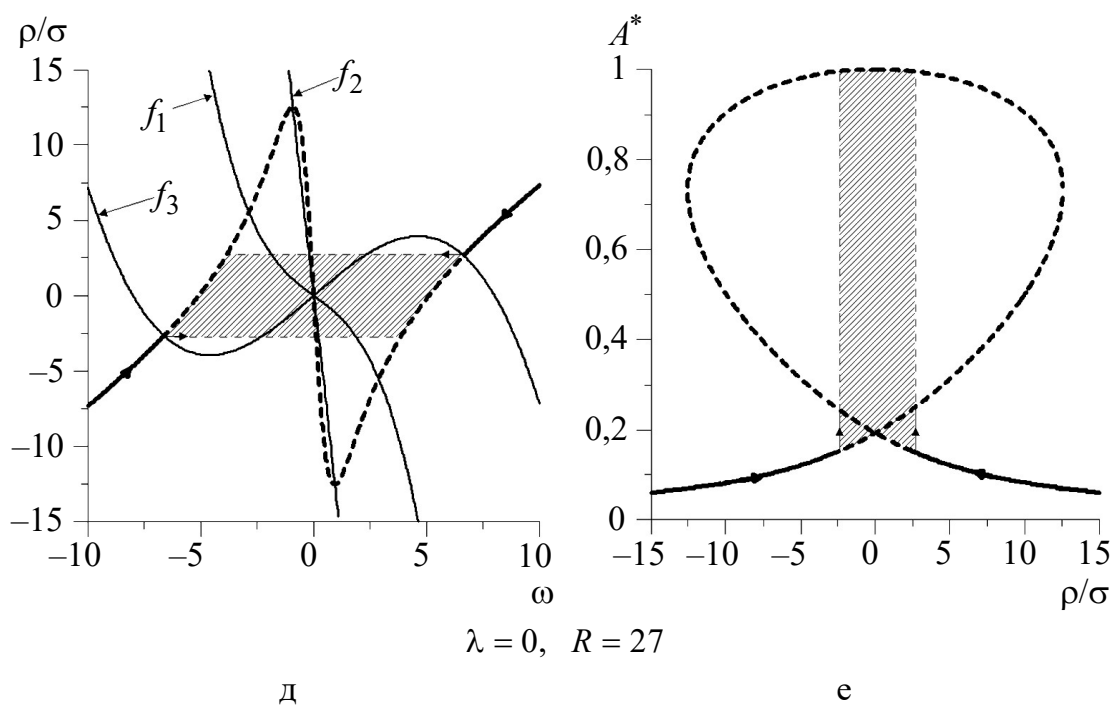

Рис. 4.11. Окончание.

Бифуркационный сценарий рождения и исчезновения динамического хаоса при прохождении гистерезисной петли для параметров системы, соответствующих малым $\Lambda$, описан в численном эксперименте 1.

Рассмотрим поведение характеристик при значительных $\Lambda$ и различных $R$. Асимметрия $\mathrm{XB}$ и РХ, малозаметная при малых $\Lambda$, становится явной при больших значениях этого параметра. На рис. 4.12 представлена галерея ХВ и РХ при $\Lambda=-3$. В этом случае ХВ является гистерезисной, в том числе и при малых $R$, а РХ уклоняется вправо (рис. 4.12,а,б). По мере возрастания параметра $R$ увеличиваются неустойчивые участки возрастающих ветвей XВ, как и соответствующие участки PX (рис. 4.12,в,г). В описанных случаях динамика системы является регулярной.

При увеличении параметра $R$ глубина гистерезиса уменьшается до нуля, а затем появляется зона неопределенности ХВ и РХ, соответствующая хаотической динамике системы (рис. 4.12,д,е). В заштрихованных областях имеет место эффект рассеяния XВ и $\mathrm{PX}$, описанный выше. Бифуркационный сценарий развития динамического хаоса при разгоне двигателя и приближении частоты враще- 
ния к собственной частоте крутильных колебаний тот же, что и в эксперименте 2 .
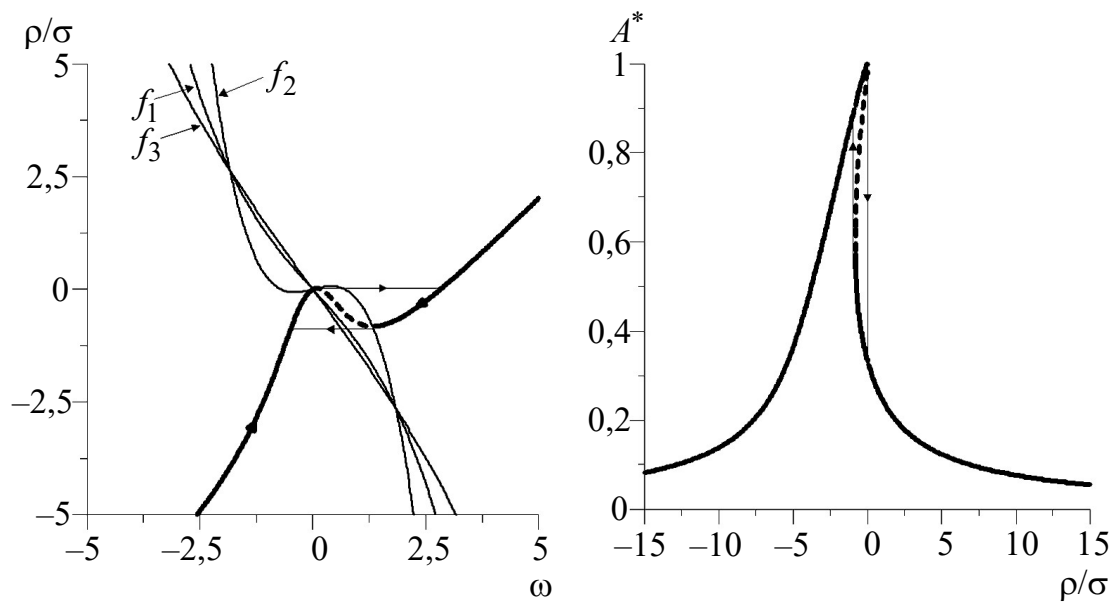

$$
\lambda=-3, \quad R=0,5
$$

a

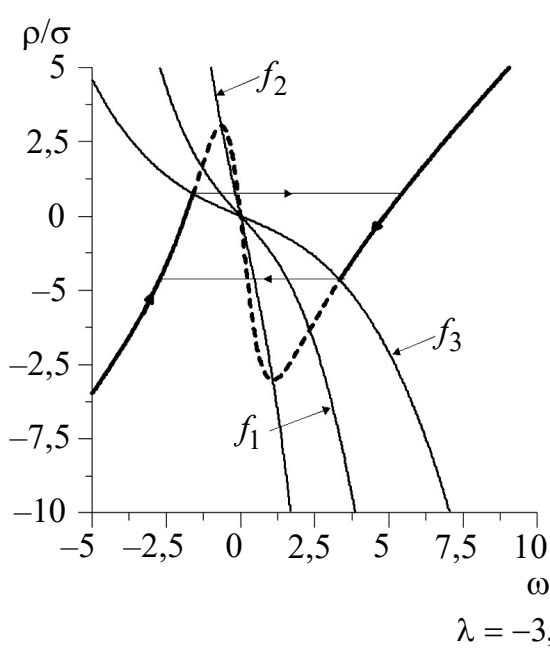

B

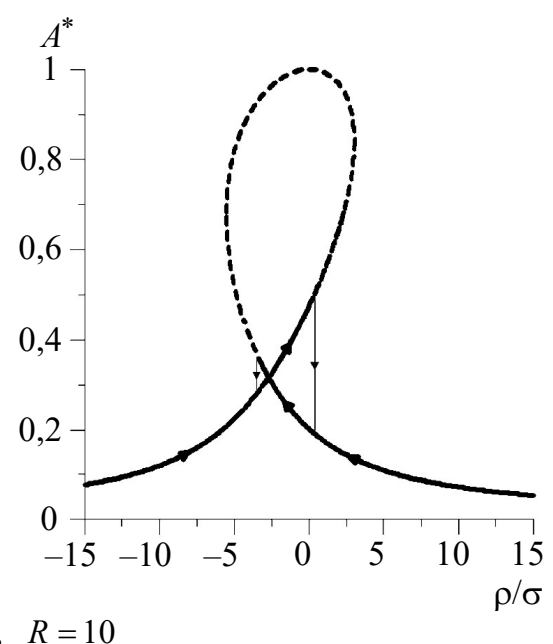

$\Gamma$

Рис. 4.12. ХВ и РХ при $\lambda=-3$ и различных значениях параметра $R$ (окончание рис. см. на с. 147). 


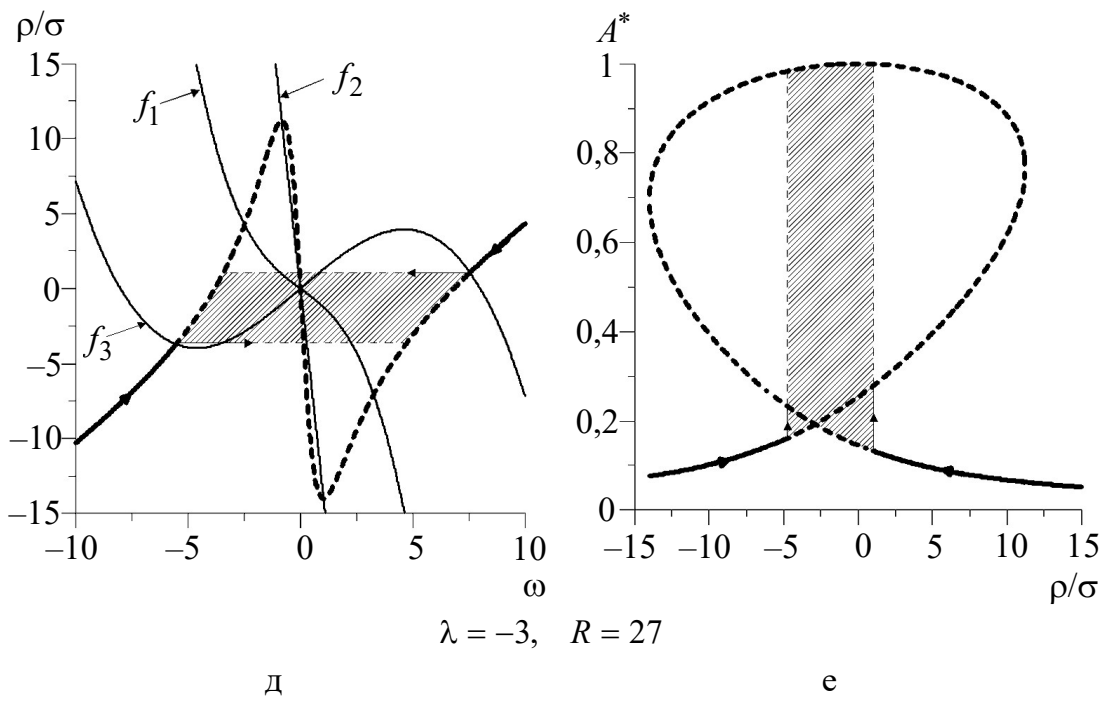

Рис. 4.12. Окончание.

Как показывает эксперимент, для любого заданного $R>R_{c}$ зоны неопределенности обеих характеристик уменьшаются при увеличении $|\Lambda|$, исчезая при некотором $\left|\Lambda^{*}\right|$. Другими словами, для всех «умеренных» значений параметра $R$ и достаточно больших $|\Lambda|$ динамика системы является регулярной во всем диапазоне изменения параметра р. На рис. 4.13 представлена галерея ХВ и РХ при $\Lambda=-20$ и различных $R$. Во всех случаях состояния равновесия системы (4.23) теряют устойчивость через обратную бифуркацию Андронова - Хопфа, а «влипающие» в состояния равновесия неустойчивые предельные циклы рождаются от петель сепаратрис седловых состояний равновесия (им соответствуют точки падающих участков ХВ). Во всех этих случаях динамика системы является регулярной и мы имеем дело с классическим эффектом Зоммерфельда. 

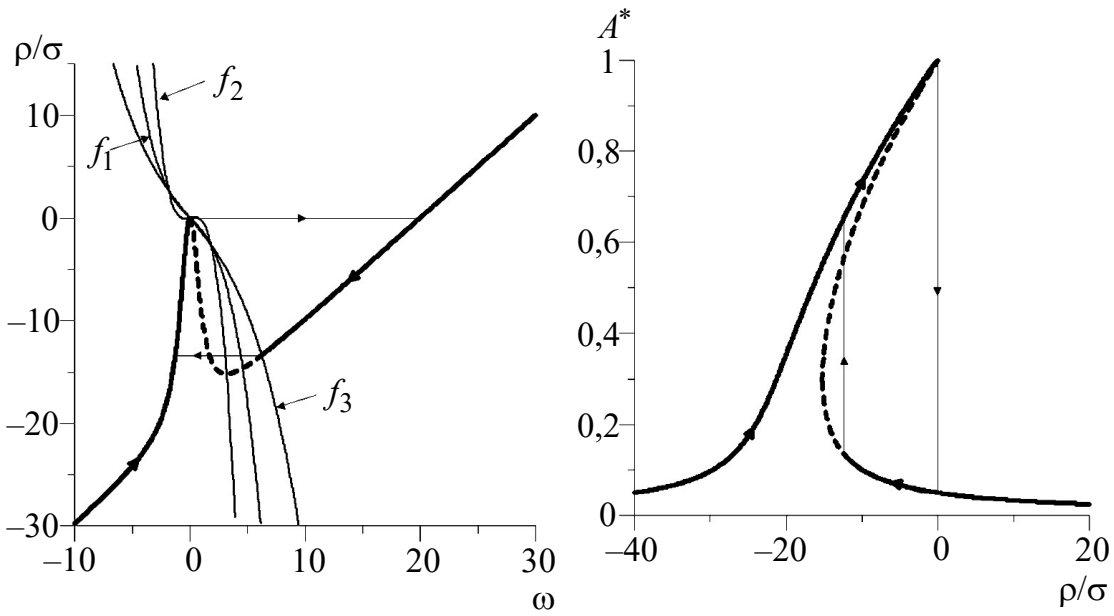

$$
\lambda=-20, \quad R=0,5
$$

a

$\rho / \sigma$

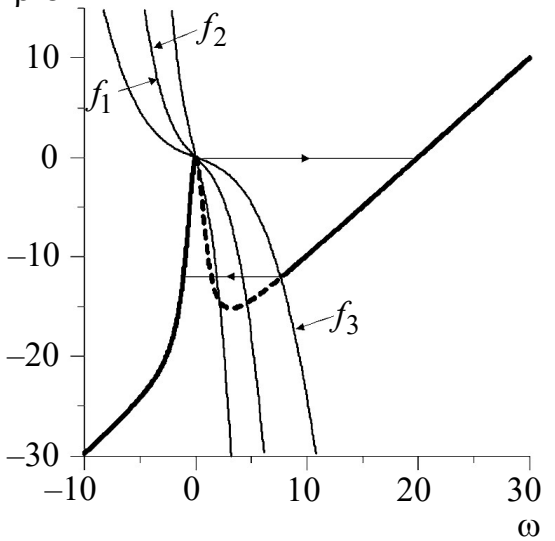

б

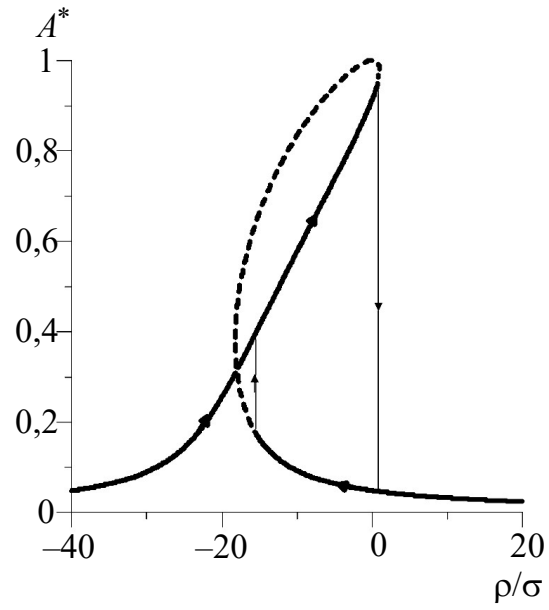

$$
\lambda=-20, \quad R=10
$$

B

$\Gamma$

Рис. 4.13. ХВ и РХ при $\lambda=-20$ и различных значениях параметра $R$ (окончание рис. см. на с. 149). 


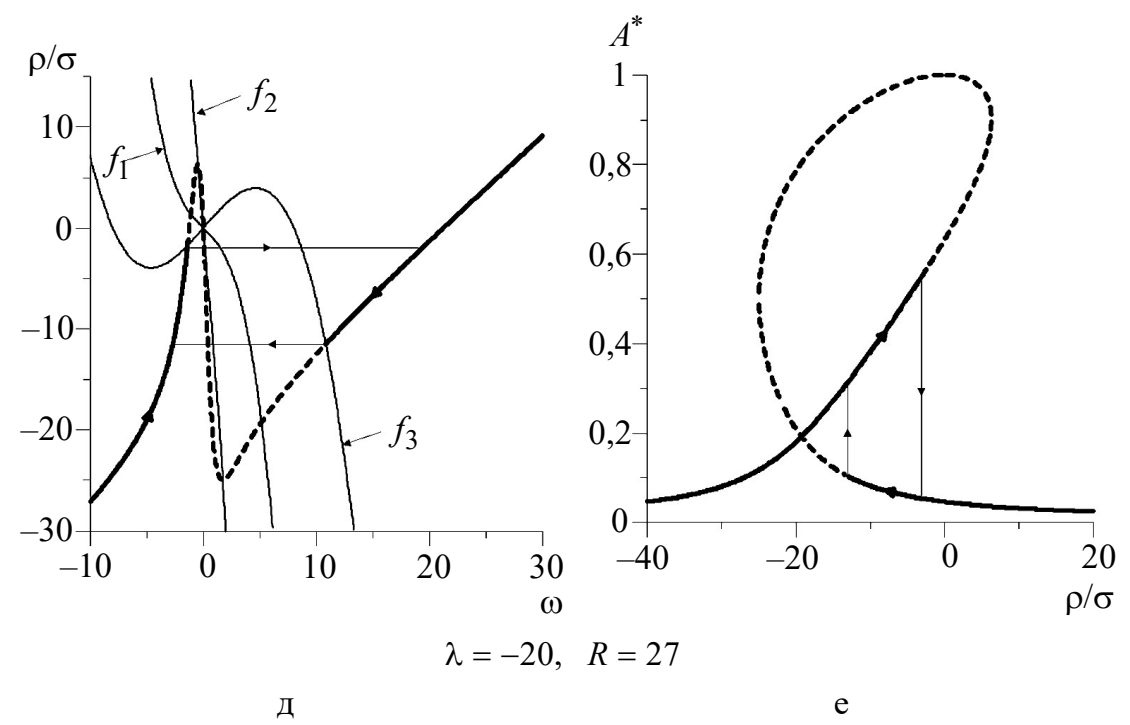

Рис. 4.13. Окончание. 


\section{Глава 5}

\section{СИНХРОНИЗАЦИЯ В ОДНОРОДНЫХ РЕШЕТКАХ}

Интерес к решеткам динамических систем (осцилляторов, ротаторов) имеет два аспекта. С одной стороны, решетки являются адекватными и удобными моделями активных сплошных сред [52, 53], для исследования, в частности, механизмов развития турбулентности [54-56]. С другой стороны, они представляют самые различные физические и биологические системы, имеющие решеточную, сетевую структуру; среди них сети СФС [57], нейронные ансамбли $[58,59]$, нейроноподобные сети по обработке информации [60-62] и др.

Эта глава посвящена исследованию устойчивости синхронизации в однородных решетках динамических систем, определяющей пространственно однородное состояние решеток. Рассматриваются диффузионно-связанные решетки различной геометрической размерности [63].

\section{1. Синхронизация в решетках динамических систем. Общие сведения}

Рассматриваем однородную решетку диффузионно-связанных динамических систем вида

$$
\begin{gathered}
\dot{\mathbf{X}}_{i}=\mathbf{F}\left(\mathbf{X}_{i}, t\right)+\varepsilon \mathbf{C}\left(\mathbf{X}_{i-1}-2 \mathbf{X}_{i}+\mathbf{X}_{i+1}\right), \\
i=\overline{1, N} .
\end{gathered}
$$

Индивидуальная система в (5.1) определена уравнением

$$
\begin{gathered}
\dot{\mathbf{X}}=\mathbf{F}(\mathbf{X}, t), \\
\mathbf{X}=\left(x_{1}, x_{2}, \ldots, x_{m}\right)^{T}, x \in R^{1}, \mathbf{F}(\mathbf{X}): R^{m} \rightarrow R^{m} .
\end{gathered}
$$

В качестве индивидуальных динамических систем могут выступать любые модели физических систем, рассмотренные в предыдущих разделах. 
Будем предполагать, что динамические свойства (5.2) нам известны. В частности, считаем известным факт, что в расширенном фазовом пространстве системы $G(\mathbf{X}, t)$ существует аттрактор $A(1)$ с максимальным ляпуновским показателем $\lambda(1)$. Последний является максимальным показателем уравнения в вариациях

$$
\dot{\mathbf{U}}=\mathbf{F}^{\prime}(\xi(t)) \mathbf{U},
$$

где $\xi(t) \in A(1), F^{\prime}(\mathbf{X})=\frac{\partial \mathbf{F}(\mathbf{X})}{\partial \mathbf{X}}-$ матрица Якоби.

Заметим, что в названных предположениях $\|\mathbf{U}\|=D \exp \lambda(1) t$, $D=$ const.

В уравнениях (5.1) матрица $C$ определяет структуру связей парциальных систем, а $\varepsilon$ является скалярным параметром.

Если одна из скалярных переменных (5.2) является циклической $x=\varphi(\bmod 2 \pi)$, то систему можно трактовать как обобщенный ротатор. Название оправдано тем, что к виду (5.2) можно привести все рассмотренные выше математические модели, если двумерные ротаторы рассматривать в совокупности с внешними воздействиями и нагрузками. Предполагаем, что функция $\mathbf{F}(\mathbf{X}, t)$ циклична по времени.

В случае граничных условий $\mathbf{X}_{0} \equiv \mathbf{X}_{1}, \mathbf{X}_{N} \equiv \mathbf{X}_{N+1}$ система (5.1) является цепочкой, а в случае $\mathbf{X}_{0} \equiv \mathbf{X}_{N}, \mathbf{X}_{1} \equiv \mathbf{X}_{N+1}$ она представляет собой кольцо.

Как будет видно из дальнейшего изложения, интересующая нас синхронизации тесно связана с существованием так называемых интегральных многообразий - особых поверхностей в фазовом пространстве связанных систем, заполненных траекториями. Этот математический объект таков, что если начальные условия системы задаются на интегральной поверхности, то изображающая точка, двигаясь по соответствующей фазовой траектории, никогда не покидает данную поверхность.

Непосредственно из системы (5.1) следует, что ее интегральным многообразием является гиперплоскость $M_{0}=\left\{\mathbf{X}_{1}=\mathbf{X}_{2}=\right.$ $\left.=\ldots=\mathbf{X}_{N-1}=\mathbf{X}_{N}\right\}$. К этому выводу мы приходим, решая уравнение 
$\mathbf{X}_{i-1}-2 \mathbf{X}_{i}+\mathbf{X}_{i+1}=0$. Следами этой гиперплоскости на координатных плоскостях соответственных переменных различных парциальных систем являются биссектрисы первого и третьего координатных углов. Заметим также, что геометрическая размерность гиперплоскости совпадает с размерностью фазового пространства индивидуальной системы.

Наш особый интерес к данному многообразию станет понятен из следующих соображений. Представим себе, что начальные условия в системе (5.1) заданы на $M_{0}$. В таком случае связанная система, как следует непосредственно из (5.1), распадается на $N$ отдельных систем вида (5.2). Кроме того, движения оказавшихся независимыми систем будут синхронизованными, поскольку это суть движения идентичных систем с идентичными начальными условиями. Другими словами, интересующая нас синхронизация соответствует движению фазовой точки по траекториям, расположенным на интегральном многообразии системы (5.1). Остается вопрос: какова природа этих траекторий? Ответ достаточно простой: поскольку проекциями данных траекторий на фазовые пространства индивидуальных систем (парциальные фазовые портреты) являются траектории парциальных систем, то и траектории на гиперплоскости $M_{0}$ индуцированы такой же системой вида (5.2).

Разумеется, задание точных начальных условий (на интегральном многообразии) находится за пределами реального эксперимента. Для решения вопроса о синхронизации должна решаться задача устойчивости интегрального многообразия.

Допустим, что интегральное многообразие устойчиво. В таком случае процесс синхронизации можно описать следующим образом. При любом начальном условии из окрестности многообразия (по крайней мере, из малой его окрестности) изображающая точка устремляется к поверхности $M_{0}$ и спустя некоторое время начинает двигаться практически по траектории аттрактора $A(1)$ системы (5.2), заданной на этом многообразии. При этом все парциальные фазовые точки в пространствах индивидуальных систем (проекции фазовой точки системы (5.1)) движутся во взаимном синхронизме по соответствующим траекториям своих парциальных фазовых портретов - по идентичным траекториям идентичных аттракторов 
$A(1)$. Другими словами, в решетке (5.1) имеет место устойчивый режим взаимной синхронизации взаимодействующих субъектов.

Синхронизация в цеепочке. Рассмотрим локальную устойчивость синхронизации как локальную устойчивость «куска» многообразия, содержащего аттрактор $A(1)$. Можно установить, что относительно трансверсалей $\mathbf{U}=\left(\mathbf{U}_{1}, \mathbf{U}_{2}, . ., \mathbf{U}_{p}\right)$ многообразия $M_{0}$, где $\mathbf{U}_{k}=\mathbf{X}_{k}-\mathbf{X}_{k+1}, \quad k=\overline{1, p}, \quad p=N-1$, линеаризованная система (5.1) сводится к одному уравнению вида

$$
\dot{\mathbf{U}}=\left(\mathbf{I}_{p} \otimes \mathbf{J}_{m}(t)-\varepsilon \mathbf{D}_{p} \otimes \mathbf{C}\right) \mathbf{U} .
$$

Здесь $J_{m}(\xi(t))$ - матрица Якоби элементарного осциллятора, $\xi(t) \in A(1), \otimes-$ символ прямого (кронекерова) произведения матриц, $\mathbf{I}_{p}$ - единичная матрица соответствующего порядка, $\mathbf{D}_{p}-$ симметрическая матрица вида

$$
\mathbf{D}_{p}=\left(\begin{array}{rrrrrrrrr}
2 & -1 & 0 & 0 & & & & & \\
-1 & 2 & -1 & 0 & & & & & \\
0 & -1 & 2 & -1 & & & 0 & \\
0 & 0 & -1 & 2 & & & & & \\
& & & & \ddots & & & & \\
& & & & & 2 & -1 & 0 & 0 \\
& & & & & -1 & 2 & -1 & 0 \\
& & & & & 0 & -1 & 2 & -1 \\
& & & & & 0 & 0 & -1 & 2
\end{array}\right) .
$$

Устойчивая изохронная синхронизация соответствует устойчивому решению $\mathbf{U}=0$ уравнения (5.3). Это уравнение обладает радикально упрощающим решение задачи свойством, которое сформулируем в виде леммы (см. также [64]).

Лемма 5.1. Пусть $\lambda_{\min }=\lambda_{1}<\lambda_{2}<\lambda_{3}<\ldots<\lambda_{p-1}<\lambda_{p}=\lambda_{\max }$ спектр собственных значений постоянной матрицы $\mathbf{D}_{p}$. В таком 
случае уравнение (5.3) в результате невырожденного преобразования $\mathbf{U}=\mathbf{S V}$ приводится к виду

$$
\begin{aligned}
& \dot{\mathbf{V}}_{j}=\left(\mathbf{J}_{m}(t)-\varepsilon \lambda_{j} \mathbf{C}\right) \mathbf{V}_{j}, \\
& j=\overline{1, p} .
\end{aligned}
$$

Доказательство. Пусть $\mathbf{S}_{0}$ - преобразующая матрица матрицы $\mathbf{D}_{p}$, a $\mathbf{S}$ - преобразующая матрица матрицы $\mathbf{D}_{p} \otimes \mathbf{C}$, т. е. $\mathbf{S}_{0}^{-1} \mathbf{D}_{p} \mathbf{S}_{0}=\mathbf{D}_{0}=\operatorname{diag}\left(\lambda_{1}, \lambda_{2}, \ldots, \lambda_{p}\right)$. Известно, что корни матрицы $\mathbf{D}_{p} \otimes \mathbf{C}$ являются всевозможными произведениями корней матрицсомножителей: $\lambda_{1} c_{1}, \lambda_{1} c_{2}, \ldots, \lambda_{1} c_{m} ; \quad \lambda_{2} c_{1}, \lambda_{2} c_{2}, \ldots, \lambda_{2} c_{m} ; \ldots ; \lambda_{p} c_{1}$, $\lambda_{p} c_{2}, \ldots, \quad \lambda_{p} c_{m}, \quad$ т. е. $\quad \mathbf{S}^{-1}\left(\mathbf{D}_{p} \otimes \mathbf{C}\right) \mathbf{S}=\mathbf{D}_{0} \otimes \mathbf{C} . \quad$ Покажем, что $\mathbf{S}=\mathbf{S}_{0} \otimes \mathbf{I}_{m}$. Применяя свойства прямого произведения [65], получаем

$$
\begin{aligned}
& \mathbf{S}^{-1}\left(\mathbf{D}_{p} \otimes \mathbf{C}\right) \mathbf{S}=\mathbf{S}^{-1}\left(\mathbf{S}_{0} \mathbf{D}_{0} \mathbf{S}_{0}^{-1} \otimes \mathbf{I}_{m} \mathbf{C} \mathbf{I}_{m}^{-1}\right) \mathbf{S}= \\
= & \mathbf{S}^{-1}\left(\mathbf{S}_{0} \otimes \mathbf{I}_{m}\right)\left(\mathbf{D}_{0} \otimes \mathbf{C}\right)\left(\mathbf{S}_{0}^{-1} \otimes \mathbf{I}_{m}\right) \mathbf{S}=\mathbf{D}_{0} \otimes \mathbf{C} .
\end{aligned}
$$

Отсюда следует, что $\mathbf{S}^{-1}\left(\mathbf{S}_{0} \otimes \mathbf{I}_{m}\right)=\mathbf{I}_{m p}$ и $\mathbf{S}=\mathbf{S}_{0} \otimes \mathbf{I}_{m}$.

Выполним в уравнении (5.3) преобразование $\mathbf{U}=\mathbf{S V}$. В результате получаем уравнение вида

$$
\dot{\mathbf{V}}=\mathbf{S}^{-1}\left(\mathbf{I}_{p} \otimes \mathbf{J}_{m}\right) \mathbf{S V}-\boldsymbol{\varepsilon} \mathbf{S}^{-1}\left(\mathbf{D}_{p} \otimes \mathbf{C}\right) \mathbf{S V} .
$$

Покажем, что матрица $\mathbf{S}^{-1}\left(\mathbf{I}_{p} \otimes \mathbf{J}_{m}\right) \mathbf{S}$ является клеточно-диагональной. Используя свойства прямого произведения матриц, получаем:

$$
\begin{aligned}
& \mathbf{S}^{-1}\left(\mathbf{I}_{p} \otimes \mathbf{J}_{m}\right) \mathbf{S}=\left(\mathbf{S}_{0}^{-1} \otimes \mathbf{I}_{m}\right)\left(\mathbf{I}_{p} \otimes \mathbf{J}_{m}\right)\left(\mathbf{S}_{0} \otimes \mathbf{I}_{m}\right)= \\
= & \left(\mathbf{S}_{0}^{-1} \mathbf{I}_{p} \mathbf{S}_{0}\right) \otimes\left(\mathbf{I}_{m} \mathbf{J}_{m} \mathbf{I}_{m}\right)=\mathbf{I}_{p} \otimes \mathbf{J}_{m}=\operatorname{diag}\left(\mathbf{J}_{m}, \mathbf{J}_{m}, \ldots, \mathbf{J}_{m}\right) .
\end{aligned}
$$

C учетом всех преобразований относительно координат вектора $\mathbf{V}$ получаем систему уравнений (5.4), эквивалентную уравнению (5.3). 
Заметим, что преобразование уравнения (5.3) к уравнению (5.4) по смыслу аналогично приведению системы линейных и линейно связанных осцилляторов к нормальным колебаниям (нормальной форме).

На базе этой леммы сформулируем следующую теорему о локальной устойчивости синхронизации.

Теорема 5.1. Пусть $\lambda(1)$ - максимальный показатель аттрактоpa $A(1)$ парциального осциллятора, а $\lambda_{\min }, \lambda_{\max }-$ минимальный и максимальный собственные значения матрицы $\mathbf{D}_{p}$. Если матрица $\mathbf{C}=\mathbf{I}$, то синхронизация в связанной системе осцилляторов устойчива при выполнении неравенства $\varepsilon>\lambda(1) / \lambda_{\min }$ и неустойчива при обратном знаке неравенства. При выполнении неравенства $\varepsilon<\lambda(1) / \lambda_{\max }$ она неустойчива по всем направлениям, трансверсальным многообразию $M_{0}$. При промежуточных значениях параметра связи синхронизация мультистабильна. Для другой из числа допустимых матрицы $\mathbf{C}$ имеют место те же неравенства с заменой $\lambda(1) \rightarrow \varepsilon^{*}(\lambda(1))$, где величина $\varepsilon^{*}$ зависит от этой матрицы.

Доказательство. Допустим, что $\mathbf{C}=\mathbf{I}$. Произведем в уравнениях (5.4) замену $\mathbf{V}_{j}=e^{-\varepsilon \lambda_{j} t} \mathbf{W}_{j}$, в результате получим систему идентичных уравнений вида $\dot{\mathbf{W}}_{j}=\mathbf{J}_{m}(t) \mathbf{W}_{j}$. По форме каждое из них является уравнением в вариациях относительно решения, соответствующего траектории аттрактора одиночного осциллятора, т. е. $\left\|\mathbf{W}_{j}\right\|=C e^{\lambda(1) t}$, где $C-$ некоторая постоянная. В силу замены переменных получаем $\left\|\mathbf{V}_{j}\right\|=C e^{\left(\lambda(1)-\varepsilon \lambda_{j}\right) t}$. Отсюда следует, что если $\varepsilon>\lambda(1) / \lambda_{j}$, то $\left\|\mathbf{V}_{j}\right\| \rightarrow 0$ при $t \rightarrow \infty$, а также $\left\|\mathbf{V}_{j}\right\| \rightarrow \infty$ при $t \rightarrow \infty$, если $\varepsilon<\lambda(1) / \lambda_{j}$. Это значит, что если $\varepsilon>\lambda(1) / \lambda_{\min }$, то все уравнения (5.4) устойчивы, а если $\varepsilon<\lambda(1) / \lambda_{\max }$, то все уравнения (5.4) неустойчивы. По лемме условия устойчивости (неустойчивости) переносятся на уравнение (5.3). При промежуточных значениях параметра $\varepsilon$ одна часть уравнений устойчива, а другая неустойчива - 
решение $\mathbf{V}=0$, а следовательно, и $\mathbf{U}=0$ имеет седловой тип, что соответствует мультистабильной или, что то же самое, частичной синхронизации.

Функция $\varepsilon^{*}(\lambda(1))$ равна нулю при $\lambda(1) \leq 0$ и возрастает при $\lambda(1)>0$. Кроме того, она зависит от структуры связей осцилляторов - элементов $c_{i}$ матрицы С. В частности, если $\mathbf{C}=\mathbf{I}$, то $\varepsilon^{*}(\lambda(1))=\lambda(1)$.

Обратимся к анализу условий устойчивости синхронизации.

Вычисление собственных значений матрицы $\mathbf{D}_{p}$ [66] для минимального корня дает $\lambda_{\min }=4 \sin ^{2} \frac{\pi}{2 N}$. После этого условие устойчивости синхронизации приобретает вид

$$
\varepsilon>\varepsilon^{*}(\lambda(1)) /\left(4 \sin ^{2} \frac{\pi}{2 N}\right) .
$$

Допустим, что аттрактор индивидуальной системы является регулярным (устойчивый предельный цикл, тор), т. е. $\lambda(1)<0$, $\varepsilon^{*}(\lambda(1))=0$. Как видим из (5.5), в этом случае синхронизация в цепочке является устойчивой при любом, в том числе и сколь угодно малом, параметре связи. Причем это имеет место для цепочек с любым числом элементов.

Совершенно иная ситуация возникает в случае, когда аттрактор индивидуальной системы является хаотическим. Тогда $\lambda(1)>0, \varepsilon^{*}(\lambda(1))>0$, тем самым правая часть (5.5) устанавливает пороговое значение параметра связи, зависящее как от степени хаотичности аттрактора, мерой которой является $\lambda(1)$, так и от числа динамических систем в цепочке $N$. При фиксированном $\varepsilon$ число систем в цепочке, для которого возможна взаимная хаотическая синхронизация, определяется неравенством

$$
N \leq N_{0}=\left[\frac{\pi}{\arccos v}\right], \quad v=1-\frac{\varepsilon^{*}(\lambda(1))}{2 \varepsilon} .
$$


В частности, если $N>>1$, то справедливы асимптотические формулы: $\varepsilon>\pi^{-2} N^{2} \varepsilon^{*}(\lambda(1)), \quad N \leq N_{0}=\left[\pi \sqrt{\varepsilon / \varepsilon^{*}}\right]$.

Синхронизачия в кольце. Рассмотрим устойчивость синхронизации динамических систем в кольце. С учетом граничных условий $\mathbf{X}_{0} \equiv \mathbf{X}_{N}, \mathbf{X}_{1} \equiv \mathbf{X}_{N+1} \quad$ относительно переменных $\mathbf{U}_{k}=\mathbf{X}_{k}-\mathbf{X}_{k+1}$, $k=\overline{1, N-1}$ - трансверсалей «куска» многообразия, содержащего хаотический аттрактор, получаем уравнение того же вида, что и (5.3), с матрицей $\mathbf{D}_{p}, p=N-1$ вида

$$
\mathbf{D}_{p}=\left(\begin{array}{ccccccccc}
3 & 0 & 1 & 1 & \ldots & 1 & 1 & 1 & 1 \\
-1 & 2 & -1 & 0 & \ldots & 0 & 0 & 0 & 0 \\
0 & -1 & 2 & -1 & \ldots & 0 & 0 & 0 & 0 \\
0 & 0 & -1 & 2 & \ldots & 0 & 0 & 0 & 0 \\
\vdots & \vdots & \vdots & \vdots & \ddots & \vdots & \vdots & \vdots & \vdots \\
0 & 0 & 0 & 0 & \ldots & 2 & -1 & 0 & 0 \\
0 & 0 & 0 & 0 & \ldots & -1 & 2 & -1 & 0 \\
0 & 0 & 0 & 0 & \ldots & 0 & -1 & 2 & -1 \\
1 & 1 & 1 & 1 & \ldots & 1 & 1 & 0 & 3
\end{array}\right) .
$$

Собственные значения матрицы $\mathbf{D}_{p}$ выражаются формулой $\lambda_{j}=4 \sin ^{2} \pi j / N, j=\overline{1, N-1}, \quad$ откуда $\quad \lambda_{\min }=4 \sin ^{2} \frac{\pi}{N}, \quad$ a $\quad \lambda_{\max }=$ $=4 \sin ^{2} \frac{\pi j^{*}}{N}$, где $j^{*}=\frac{N}{2}$, если $N$ четно, и $j^{*}=\frac{N+1}{2}$, если $N$ нечетно.

Таким образом, изохронная синхронизация в кольце динамических систем является устойчивой, если выполняется неравенство $\varepsilon>\varepsilon^{*} / 4 \sin ^{2} \frac{\pi}{N}$, а если $\varepsilon<\varepsilon^{*} / 4 \sin ^{2} \frac{\pi j}{N}$, то она является неустойчивой.

Синхронизащия в двумерной решетке. Рассмотрим устойчивость синхронизации динамических систем в двумерной прямоугольной решетке, моделируемой дифференциальными уравнениями вида 


$$
\begin{gathered}
\dot{\mathbf{X}}_{i j}=\mathbf{F}\left(\mathbf{X}_{i j}\right)-\varepsilon \mathbf{C}\left(4 \mathbf{X}_{i j}-\mathbf{X}_{i-1 j}-\mathbf{X}_{i+1 j}-\mathbf{X}_{i j-1}-\mathbf{X}_{i j+1}\right), \\
i=1,2, \ldots N_{1}, \quad j=1,2, \ldots N_{2}
\end{gathered}
$$

с граничными условиями: $\mathbf{X}_{0 j}=\mathbf{X}_{1 j}, \quad \mathbf{X}_{N_{1}+1 j}=\mathbf{X}_{N_{1 j}}, \quad \mathbf{X}_{i 0}=\mathbf{X}_{i 1}$, $\mathbf{X}_{i N_{2}+1}=\mathbf{X}_{i N_{2}}$.

На примере цепочки мы увидели, что исследование устойчивости синхронизации в конечном итоге свелось к исследованию собственных значений матрицы $\mathbf{D}_{p}$, отвечающей за связь индивидуальных систем. Очевидно, что к тому же должна свестись задача устойчивости и в случае уравнений (5.6). Задачу поиска соответствующей матрицы решим путем синтеза двумерной решетки из соответствующих цепочек как «элементов» решетки.

Рассмотрим прямоугольную решетку как «параллельное» соединение цепочек. Для этого снабдим в (5.1) векторы элементарного осциллятора вторым индексом, введем векторы $\mathbf{Y}_{j}=$ $=\operatorname{col}\left(\mathbf{X}_{1 j}, \mathbf{X}_{2 j}, \ldots, \mathbf{X}_{N_{1 j}}\right), \quad \Phi\left(Y_{j}\right)=\operatorname{col}\left(\mathbf{F}\left(\mathbf{X}_{1 j}\right), \mathbf{F}\left(\mathbf{X}_{2 j}\right), \ldots, \mathbf{F}\left(\mathbf{X}_{N_{1 j}}\right)\right)$ и запишем произвольную цепочку (систему (5.1)) одним уравнением:

$$
\dot{\mathbf{Y}}_{j}=\Phi\left(\mathbf{Y}_{j}\right)-\varepsilon\left(\mathbf{B}_{N_{1}} \otimes \mathbf{C}\right) \mathbf{Y}_{j}
$$

где $\mathbf{B}_{N_{1}}-$ диффузионная матрица вида

$$
\mathbf{B}_{N_{1}}=\left\|\begin{array}{ccccccc}
1 & -1 & 0 & \cdots & \cdots & 0 & 0 \\
-1 & 2 & -1 & \ddots & \cdots & 0 & 0 \\
0 & -1 & 2 & \ddots & \ddots & 0 & 0 \\
\vdots & \ddots & \ddots & \ddots & \ddots & \ddots & \vdots \\
\vdots & \vdots & \ddots & \ddots & 2 & -1 & 0 \\
0 & 0 & 0 & \ddots & -1 & 2 & -1 \\
0 & 0 & 0 & \cdots & 0 & -1 & 1
\end{array}\right\| .
$$

Поскольку при параллельном соединении цепочек все элементы вектора $\mathbf{Y}_{j}$ соединяются с соответствующими элементами соседних векторов $\mathbf{Y}_{j-1}$ и $\mathbf{Y}_{j+1}$, то матрицей синтеза решетки из эле- 
ментов (5.7) является единичная матрица $\mathbf{I}_{N_{1}}$. С учетом сказанного и (5.7) уравнения решетки принимают вид

$$
\begin{aligned}
& \dot{\mathbf{Y}}_{j}=\Phi\left(\mathbf{Y}_{j}\right)-\varepsilon\left(\mathbf{B}_{N_{1}} \otimes \mathbf{C}\right) \mathbf{Y}_{j}-\varepsilon\left(\mathbf{I}_{N_{1}} \otimes \mathbf{C}\right)\left(-\mathbf{Y}_{j-1}+2 \mathbf{Y}_{j}-\mathbf{Y}_{j+1}\right), \\
& j=1,2, \ldots N_{2} .
\end{aligned}
$$

Граничные условия в системе (5.8): $\mathbf{Y}_{0}=\mathbf{Y}_{1}, \mathbf{Y}_{N_{2}}=\mathbf{Y}_{N_{2}+1}$.

Произведя матричные операции и записав эту систему относительно элементарных осцилляторов, можно убедиться в эквивалентности систем (5.8) и (5.6). Обратим внимание на сходство записи уравнений (5.8) и (5.1).

В отличие от предыдущего раздела, где линеаризация велась по переменным $\mathbf{U}_{i}=\mathbf{X}_{i}-\mathbf{X}_{i+1}$ (трансверсали многообразия), здесь линеаризацию уравнений (5.8) проведем стандартно - по переменным $\mathbf{U}_{i j}=\mathbf{X}_{i j}-\xi(t)$ в окрестности решения $\mathbf{X}_{i j}=\xi(t) \in A(1)$.

После свертывания линеаризованной системы в одно уравнение получаем

$$
\dot{\mathbf{U}}=\mathbf{I}_{N_{1} N_{2}} \otimes \mathbf{J}_{m}(\xi) \mathbf{U}-\varepsilon\left(\mathbf{I}_{N_{2}} \otimes \mathbf{B}_{N_{1}}+\mathbf{B}_{N_{2}} \otimes \mathbf{I}_{N_{1}}\right) \otimes \mathbf{C U} .
$$

Заметим, что способ линеаризации принципиально не меняет сути дела. Формальная разница состоит в том, что в данном случае размерность матрицы $\mathbf{D}_{p}=\mathbf{I}_{N_{2}} \otimes \mathbf{B}_{N_{1}}+\mathbf{B}_{N_{2}} \otimes \mathbf{I}_{N_{1}}$ на единицу больше той, которая получилась бы по первому образцу, и в ее спектре имеется один нулевой корень. Все другие собственные значения положительны и совпадают с собственными значениями матрицы, получающейся в первом случае (матрицы связаны эквивалентными преобразованиями). Не вдаваясь более в подробности, далее нулевой корень не принимаем во внимание.

Как видим, уравнение (5.9) подпадает под сформулированную выше лемму. Следовательно, устойчивость синхронизации определяется корнями матрицы $\mathbf{D}_{p}=\mathbf{I}_{N_{2}} \otimes \mathbf{B}_{N_{1}}+\mathbf{B}_{N_{2}} \otimes \mathbf{I}_{N_{1}}$.

Исследование этой матрицы показывает, что ее минимальное собственное значение выражается уже знакомой по предыдущему разделу формулой $\lambda_{\min }=4 \sin ^{2} \frac{\pi}{2 N}$, где $N=\max \left\{N_{1}, N_{2}\right\}$. Получа- 
ем интересный и неожиданный результат: условие устойчивости синхронизации выражается неравенством $\varepsilon>\varepsilon^{*}(\lambda(1)) /\left(4 \sin ^{2} \frac{\pi}{2 N}\right)$, совпадающим с условием устойчивости синхронизации в цепочке, «длина» которой равна наибольшей «стороне» прямоугольной решетки осцилляторов.

Синхронизация в трехмерной решетке. Рассматриваем устойчивость пространственно однородной структуры в трехмерной решетке динамических систем, моделируемой уравнениями вида

$$
\begin{gathered}
\dot{\mathbf{X}}_{i j k}=\mathbf{F}\left(\mathbf{X}_{i j k}\right)- \\
-\varepsilon \mathbf{C}\left(6 \mathbf{X}_{i j k}-\mathbf{X}_{i-1 j k}-\mathbf{X}_{i+1 j k}-\mathbf{X}_{i j-1 k}-\mathbf{X}_{i j+1 k}-\mathbf{X}_{i j k-1}-\mathbf{X}_{i j k+1}\right), \\
i=1,2, \ldots N_{1}, \quad j=1,2, \ldots N_{2}, \quad k=1,2, \ldots N_{3} .
\end{gathered}
$$

Для нахождения нужной матрицы поступаем аналогично двумерному случаю, рассматривая трехмерную решетку в виде «параллельного» соединения слоев - двумерных решеток вида (5.6). Снабдим в (5.8) каждый элементарный осциллятор третьим индексом и запишем уравнение произвольного слоя (систему (5.8)) в виде одного уравнения:

$$
\dot{\mathbf{Z}}_{k}=\mathrm{H}\left(\mathbf{Z}_{k}\right)-\varepsilon\left(\mathbf{I}_{N_{2}} \otimes \mathbf{B}_{N_{1}}+\mathbf{B}_{N_{2}} \otimes \mathbf{I}_{N_{1}}\right) \otimes \mathbf{C} \mathbf{Z}_{k} .
$$

Рассматривая теперь трехмерную решетку как «параллельное соединение слоев», записываем ее в следующей форме:

$$
\begin{gathered}
\dot{\mathbf{Z}}_{k}=\mathrm{H}\left(\mathbf{Z}_{k}\right)-\varepsilon\left(\mathbf{I}_{N_{2}} \otimes \mathbf{B}_{N_{1}}+\mathbf{B}_{N_{2}} \otimes \mathbf{I}_{N_{1}}\right) \otimes \mathbf{C} \mathbf{Z}_{k}- \\
-\varepsilon\left(\mathbf{I}_{N_{1} N_{2}} \otimes \mathbf{C}\right)\left(-\mathbf{Z}_{k-1}+2 \mathbf{Z}_{k}-\mathbf{Z}_{k+1}\right), \\
k=1,2, \ldots N_{3} .
\end{gathered}
$$

Граничные условия в системе: $\mathbf{Z}_{0}=\mathbf{Z}_{1}, \mathbf{Z}_{N_{3}}=\mathbf{Z}_{N_{3}+1}$.

Линеаризация полученной системы в окрестности решения $\mathbf{X}_{i j k}=\xi(t) \in A(1)$ и последующее ее свертывание к одному уравнению дает следующий результат: 


$$
\begin{gathered}
\dot{\mathbf{U}}=\mathbf{I}_{N_{1} N_{2} N_{3}} \otimes \mathbf{J}_{m}(\xi) \mathbf{U}- \\
-\varepsilon\left(\mathbf{I}_{N_{3}} \otimes \mathbf{I}_{N_{2}} \otimes \mathbf{B}_{N_{1}}+\mathbf{I}_{N_{3}} \otimes \mathbf{B}_{N_{2}} \otimes \mathbf{I}_{N_{1}}+\mathbf{B}_{N_{3}} \otimes \mathbf{I}_{N_{1} N_{2}}\right) \otimes \mathbf{C U} .
\end{gathered}
$$

Как видим, данное уравнение имеет нужный вид уравнения леммы. Следовательно, устойчивость синхронизации сводится к определению собственных значений матрицы $\mathbf{D}_{p}=$ $=\mathbf{I}_{N_{3}} \otimes \mathbf{I}_{N_{2}} \otimes \mathbf{B}_{N_{1}}+\mathbf{I}_{N_{3}} \otimes \mathbf{B}_{N_{2}} \otimes \mathbf{I}_{N_{1}}+\mathbf{B}_{N_{3}} \otimes \mathbf{I}_{N_{1} N_{2}}$.

При исследовании данной матрицы также обнаруживается отмеченный выше факт: минимальный корень матрицы $\mathbf{D}_{p}$ равен $4 \sin ^{2} \frac{\pi}{2 N}$, где $N=\max \left\{N_{1}, N_{2}, N_{3}\right\}$. Отсюда получаем условие устойчивости синхронизации, которое имеет уже известный по предыдущему материалу вид: $\varepsilon>\varepsilon^{*}(\lambda(1)) /\left(4 \sin ^{2} \frac{\pi}{2 N}\right)$, где $N=\max \left\{N_{1}, N_{2}, N_{3}\right\}$. Выводы относительно влияния параметров аттракторов индивидуальной динамической системы и параметров решетки на устойчивость синхронизации будут такими же, как и в случае цепочки.

\section{2. Синхронизация вращений в цепочке и кольце диффузионно-связанных автономных и неавтономных ротаторов}

Исследуем процессы синхронизации в системе связанных ротаторов вида

$$
\begin{aligned}
& I \ddot{\varphi}_{i}+\dot{\varphi}_{i}+\sin \varphi_{i}=\gamma+\varepsilon\left(\dot{\varphi}_{i-1}-2 \dot{\varphi}_{i}+\dot{\varphi}_{i+1}\right)+A \sin \psi, \\
& \dot{\psi}=\omega_{0}, \\
& i=\overline{1, N}
\end{aligned}
$$

с граничными условиями двух типов: а) $\varphi_{0} \equiv \varphi_{1}, \varphi_{N} \equiv \varphi_{N+1}$, соответствующих цепочке, и б) $\varphi_{0} \equiv \varphi_{N}, \varphi_{N+1} \equiv \varphi_{1}-$ кольцу. 
Переменные и параметры в системе (5.12) безразмерны, а сама она задана в тороидальном фазовом пространстве $G\left(\varphi_{1}, \ldots, \varphi_{N}, \psi, \dot{\varphi}_{1}, \ldots, \dot{\varphi}_{N}\right)=T^{N+1} \times R^{N}$. Параметры $I>0, \gamma>0$ имеют смысл момента инерции и постоянного движущего момента ротатора, $A, \omega_{0}$ - амплитуда и частота внешнего воздействия, $\varepsilon-$ параметр связи ротаторов.

Приведем примеры физических систем, динамика которых моделируется уравнениями (5.12).

Пример 1. На рис. 5.1 изображена цепочка маятников Фруда [67]. Муфты идентичных маятников насажены на вал, который приводится во вращательное движение с постоянной частотой вращения $\Omega=\omega$ или во вращательно-колебательное движение с частотой $\Omega=\omega+B \sin \Omega_{0} t$. Между всеми муфтами и валом, а также между всеми соприкасающимися муфтами имеется вязкое трение.

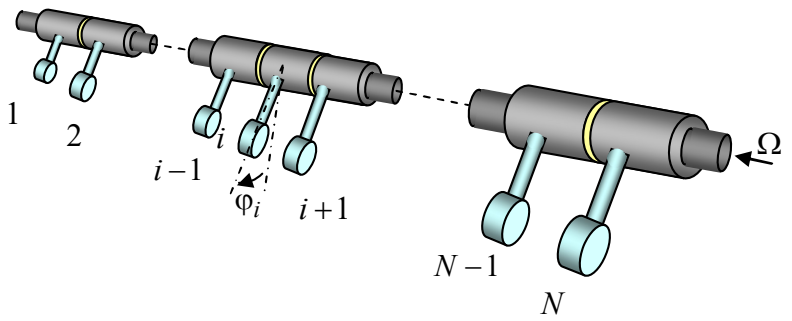

Рис. 5.1. Цепочка маятников Фруда.

В физических переменных и параметрах уравнения движения цепочки имеют вид:

$$
\begin{aligned}
& m l^{2} \ddot{\varphi}_{i}+d \dot{\varphi}_{i}+m g l \sin \varphi_{i}=R\left(\omega+B \sin \psi-\dot{\varphi}_{i}\right)+ \\
& +\lambda\left(\dot{\varphi}_{i-1}-\dot{\varphi}_{i}\right)+\lambda\left(\dot{\varphi}_{i+1}-\dot{\varphi}_{i}\right), \\
& \dot{\psi}=\Omega_{0}, \\
& i=\overline{1, N} .
\end{aligned}
$$

Здесь: $m, l$ - приведенные масса и длина маятника; $d$ - постоянный коэффициент вязкости среды; $R\left(\omega+B \sin \psi-\dot{\varphi}_{i}\right)-$ момент силы вязкого трения со стороны вала; $\lambda\left(\dot{\varphi}_{i-1}-\dot{\varphi}_{i}\right), \lambda\left(\dot{\varphi}_{i+1}-\dot{\varphi}_{i}\right)-$ мо- 
менты сил трения со стороны соседних маятников; $R, \lambda-$ постоянные коэффициенты.

Разделив уравнения на $m g l$ и переходя к безразмерному времени $\frac{m g l}{d+R} t=\tau$, получаем уравнения (5.12). Связь размерных параметров с безразмерными параметрами модели осуществляется по формулам

$$
I=\frac{l}{g}\left(\frac{m g l}{d+R}\right)^{2}, \gamma=\frac{R \omega}{m g l}, \varepsilon=\frac{\lambda}{d+R}, \omega_{0}=\Omega_{0} \frac{d+R}{m g l}, A=\frac{R B}{m g l} .
$$

Пример 2. Напомним, что состояние сверхпроводящего перехода описывается двумя переменными: $\varphi$ - разность фаз квантовомеханических функций порядка и $\dot{\varphi}=V$ - разность потенциалов сверхпроводников (соотношение Джозефсона в обезразмеренном времени). В резистивной модели в безразмерных переменных и параметрах отдельный неавтономный сверхпроводящий переход описывается маятниковым уравнением (см. разд. 1.1).

На рис. 5.2 изображена цепочка диссипативно-связанных сверхпроводящих переходов с равномерной инжекцией постоянного тока, находящихся во внешнем СВЧ-поле.

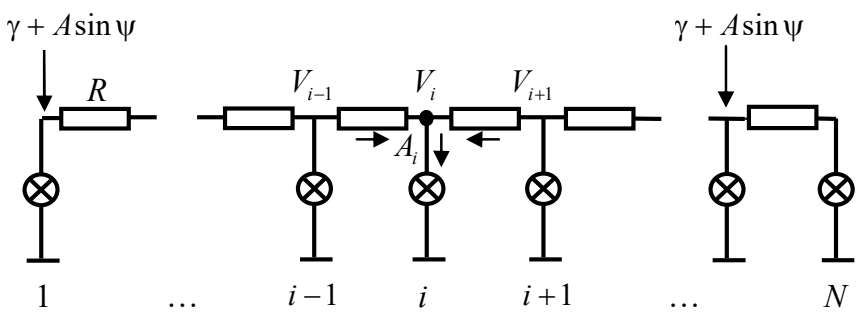

Рис. 5.2. Цепочка сверхпроводящих переходов.

Уравнения для токов в узлах $A_{i}$ и соотношение Джозефсона определяют динамическую систему цепочки: 


$$
\begin{aligned}
& c \ddot{\varphi}_{i}+\dot{\varphi}_{i}+\sin \varphi_{i}=\gamma+\frac{1}{r}\left(\dot{\varphi}_{i-1}-\dot{\varphi}_{i}\right)+\frac{1}{r}\left(\dot{\varphi}_{i+1}-\dot{\varphi}_{i}\right)+A \sin \psi, \\
& \dot{\psi}=\omega_{0}, \\
& i=\overline{1, N}
\end{aligned}
$$

с граничными условиями $\varphi_{0} \equiv \varphi_{1}, \varphi_{N} \equiv \varphi_{N+1}$. Безразмерные параметры: $c$ - электроемкость перехода, $\gamma$ - постоянный ток внешнего источника, $A, \omega_{0}$ - амплитуда и частота внешнего СВЧ-поля, $r$ сопротивление связи. Полагая $1 / r=\varepsilon$ (безразмерная проводимость), получаем уравнения (5.12).

Пример 3. На рис. 5.3 изображена блок-схема кольца диссипативно-связанных сверхпроводящих переходов. Записывая физические уравнения каждого из них, получаем динамическую систему (5.13) с периодическими условиями $\varphi_{0} \equiv \varphi_{N}, \varphi_{N+1} \equiv \varphi_{1}$. Отсчет порядкового номера перехода начинается от произвольного элемента.

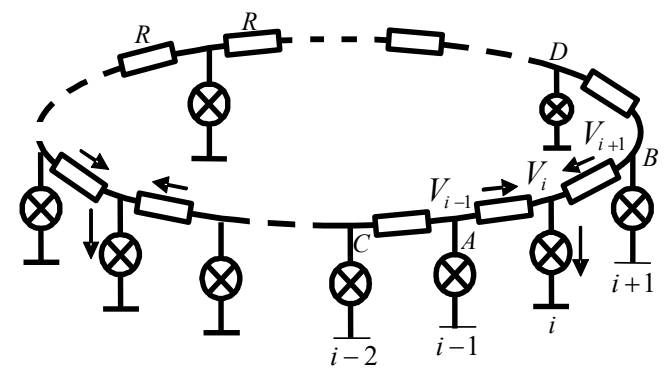

Рис. 5.3. Кольцо диссипативно-связанных джозефсоновских переходов.

Из сравнения уравнений физических систем, представленных в примерах 1 и 2, получаем следующую электромеханическую аналогию величин: $V$ (напряжение) $\sim \dot{\varphi}=\omega$ (частота вращения), $1 / R$ (проводимость связи) $\sim \varepsilon$ (коэффициент вязкого трения), $J$ (ток) $\sim M$ (момент). Выражение для тока связи: $\left(V_{i+1}-V_{i}\right) / R=J_{i+1}$ (закон Ома), текущего от $(i+1)$-го перехода к $i$-му, эквивалентно выражению для момента силы вязкого трения, действующей со стороны $(i+1)$-го маятника на $i$-й. Условие $\left\langle\dot{\varphi}_{i}\right\rangle_{t}=0$ соответствует 
состоянию равновесия и колебательным движениям $i$-го маятника в цепочке на рис. 5.1, а также сверхпроводящему состоянию соответствующего перехода. Условие $\left\langle\dot{\varphi}_{i}\right\rangle_{t} \neq 0$ соответствует вращательным движениям маятника и резистивному состоянию, или, что то же самое, режиму генерации перехода.

$1^{0}$. Автономная цุепочка и кольц̧о. Полагая в уравнениях (5.12) $A=0$ и преобразуя время $\sqrt{I^{-1}} \tau=\tau_{n}$, получаем систему вида

$$
\begin{aligned}
& \ddot{\varphi}_{i}+\lambda \dot{\varphi}_{i}+\sin \varphi_{i}=\gamma+\sigma\left(\dot{\varphi}_{i-1}-2 \dot{\varphi}_{i}+\dot{\varphi}_{i+1}\right), \\
& i=\overline{1, N},
\end{aligned}
$$

где $\lambda=\sqrt{I^{-1}}, \sigma=\varepsilon \sqrt{I^{-1}}$. Граничные условия: $\varphi_{0}=\varphi_{1}, \varphi_{N}=\varphi_{N+1}-$ для цепочки, $\varphi_{0}=\varphi_{N}, \varphi_{N+1}=\varphi_{1}-$ для кольца.

Некоторые общие свойства системы (5.13).

1) В фазовом пространстве системы $G(\varphi, \dot{\varphi})=T^{N} \times R^{N}$ отсутствуют замкнутые траектории колебательного типа ( $O$ траектории). Свойство устанавливается при помощи периодической функции Ляпунова: рассматриваем функцию (квадратичная форма плюс интеграл от нелинейности) вида

$$
V=\sum_{i=1}^{N}\left(\frac{1}{2} \dot{\varphi}_{i}^{2}+\int_{\varphi_{0 i}}^{\varphi_{i}}\left(\sin \varphi_{i}-\gamma\right) d \varphi_{i}\right),
$$

ее производная, вычисленная в силу уравнений (5.13), $\dot{V}=-\sum_{i=1}^{N}\left(\lambda \dot{\varphi}_{i}^{2}+\sigma\left(\dot{\varphi}_{i}-\dot{\varphi}_{i+1}\right)^{2}\right) \leq 0$.

Знакоотрицательность производной во всем фазовом пространстве $G(\varphi, \dot{\varphi})$ доказывает свойство.

Таким образом, возможными аттракторами системы (5.13) могут являться: состояния равновесия, вращательно-колебательные и вращательные предельные циклы, торы и, может быть, хаотические аттракторы с вращающейся фазой.

2) В фазовом пространстве системы существует «главное» интегральное многообразие 
$M_{0}=\left\{\varphi_{1}=\varphi_{2}=\ldots=\varphi_{N}, \dot{\varphi}_{1}=\dot{\varphi}_{2}=\ldots=\dot{\varphi}_{N}\right\} \quad-\quad$ цилиндр $\quad(\varphi, \dot{\varphi})$, заполненный фазовыми траекториями уравнения одиночного ротатора - уравнения маятника (см. разд. 5.1):

$$
\ddot{\varphi}+\lambda \dot{\varphi}+\sin \varphi=\gamma .
$$

О свойствах уравнения маятника подробно см. разд. 1.1.

Исследуем стационарные пространственно однородные динамические режимы цепочки (синхронизацию), соответствующие устойчивым предельным множествам фазовых траекторий, лежащим на главном многообразии $M_{0}$. Устойчивое состояние равновесия $O_{1}(\arcsin \gamma, 0)$ соответствует покоящимся маятникам Фруда, расположенным под одинаковым углом к вертикали; предельный цикл соответствует синфазному вращению всех маятников цепочки. На примере данной системы повторим доказательство того, что эти предельные множества являются устойчивыми по направлениям, трансверсальным $M_{0}$.

Заменой переменных $u_{i}=\varphi_{i}-\varphi_{i+1}, \quad \dot{u}_{i}=v_{i} \quad$ приведем уравнения (5.13) к многообразию $M_{0}$. В результате получаем:

$$
\begin{aligned}
& \dot{u}_{i}=v_{i}, \\
& \dot{v}_{i}=-\lambda v_{i}-\left(\cos \xi_{i}\right) u_{i}+\sigma\left(v_{i-1}-2 v_{i}+v_{i+1}\right), \\
& i=\overline{1, N-1} .
\end{aligned}
$$

Граничные условия в (5.14): $v_{0} \equiv 0, v_{N} \equiv 0$. При переходе к системе (3.16) использована теорема Лагранжа: $\xi_{i} \in\left(\varphi_{i}, \varphi_{i+1}\right)$.

Заметим, что если $\xi_{1}=\varphi_{1}=\varphi_{2}=\xi_{2}=\ldots=\varphi_{N}=\varphi$, где $\varphi(t)-$ произвольное решение и $(\varphi, \dot{\varphi}) \in M_{0}$, то система (5.14) является системой в вариациях относительно данного многообразия.

Запишем систему (5.14) одним уравнением вида

$$
\dot{\mathbf{U}}=\left(\mathbf{I}_{N-1} \otimes \mathbf{J}_{0}(\xi)-\sigma \mathbf{D}_{N-1} \otimes \mathbf{C}\right) \mathbf{U},
$$

где $\mathbf{U}=\left(u_{1}, v_{1}, u_{2}, v_{2} . ., u_{N-1}, v_{N-1}\right)^{T}, \mathbf{J}_{0}=\left(\begin{array}{cc}0 & 1 \\ -\cos \xi & -\lambda\end{array}\right), \mathbf{C}=\left(\begin{array}{ll}0 & 0 \\ 0 & 1\end{array}\right)$, 


$$
\mathbf{D}_{N-1}=\left(\begin{array}{rrrrrrrrrr}
2 & -1 & 0 & 0 & & & & & \\
-1 & 2 & -1 & 0 & & & & & \\
0 & -1 & 2 & -1 & & & 0 & & \\
0 & 0 & -1 & 2 & & & & & \\
& & & & \ddots & & & & \\
& & & & & 2 & -1 & 0 & 0 \\
& & & & & -1 & 2 & -1 & 0 \\
& & & & & 0 & -1 & 2 & -1 \\
& & & & & 0 & 0 & -1 & 2
\end{array}\right) .
$$

По лемме 5.1 уравнение (5.15) распадается на $N-1$ двумерных систем относительно $\left(x_{i}, y_{i}\right)^{T}=\mathbf{V}_{i}$ вида

$$
\begin{aligned}
& \dot{x}_{i}=y_{i}, \\
& \dot{y}_{i}=-\lambda y_{i}-(\cos \varphi) x_{i}-\sigma \lambda_{i} y_{i}, \\
& i=\overline{1, N-1,}
\end{aligned}
$$

где $\lambda_{i}=4 \sin ^{2} \pi i / 2 N, i=\overline{1, N-1}-$ собственные значения матрицы $\mathbf{D}_{N-1}$.

Если $\sigma=0$, то каждая из этих систем - устойчивая система в вариациях (невозмущенная система) относительно решения, соответствующего одной из устойчивых предельных траекторий уравнения маятника (устойчивое состояние равновесия, устойчивый предельный цикл). С другой стороны, если $\sigma \neq 0$, то при любом фиксированном $t=t_{0}$ имеется правый поворот векторного поля каждой из систем (5.16) на траекториях невозмущенной системы. Другими словами, поскольку все траектории невозмущенной системы (в расширенном фазовом пространстве) входят внутрь и не покидают некоторый цилиндр $Z\left\{t>t_{0}, x^{2}+y^{2} \leq R^{2}\left(t_{0}\right)\right\}$, $\lim R\left(t_{0}\right)=0$, то это относится и к траекториям возмущенной сис$t_{0} \rightarrow \infty$

темы. То есть из устойчивости невозмущенной системы следует 
устойчивость каждой системы из (5.16). Следовательно, решение $\mathbf{V}=0$, а значит, и $\mathbf{U}=0$ устойчиво.

Физически этот факт понятен: в устойчивую систему вносится дополнительная диссипация, что, естественно, только улучшает ее устойчивость.

Таким образом, пространственно однородный динамический режим цепочки (синхронизация) устойчив при любом, в том числе и сколь угодно малом, уровне диффузионной связи, что подтверждает результат, полученный в предыдущем параграфе.

Обратимся к бифуркационной диаграмме (см. рис. 1.1). Для параметров из области (1) и начальных условий, заданных в общем случае в малой окрестности многообразия, пространственно однородным состоянием цепочки является состояние равновесия: все маятники цепочки с течением времени приходят в состояние равновесия, т. е. висят под одним углом. В области параметров (2) в зависимости от начальных условий может реализоваться как состояние равновесия, так и пространственно однородный режим синхронных вращений маятников цепочки. Наконец, в области параметров (3) при любых начальных условиях, заданных в окрестности многообразия, реализуется пространственно однородный режим синфазных вращений.

Для автономного кольца справедливы те же самые выводы, что и для цепочки ротаторов.

$2^{0}$. Неавтономная цеепочка и кольцио. Исследуем динамические свойства неавтономной системы (5.12) методом усреднения в зоне параметров главного резонанса.

Следуя алгоритмам преобразования систем связанных ротаторов, при помощи замены

$$
\dot{\varphi}_{i}=\omega_{0}+\mu F_{i}\left(\varphi_{i}, \psi, \xi\right), F_{i}=\frac{\cos \varphi_{i}}{\omega_{0}}-\frac{A \cos \psi}{\omega_{0}}+\xi_{i}, \quad \eta_{i}=\varphi_{i}-\psi
$$

получаем эквивалентную (5.12) систему в стандартной форме вида

$$
\begin{aligned}
& \dot{\xi}_{i}=\mu\left(-F_{i} \frac{\partial F_{i}}{\partial \varphi_{i}}-F_{i}+\sigma\left(F_{i-1}-2 F_{i}+F_{i+1}\right)+\Delta\right), \\
& \dot{\eta}_{i}=\mu F_{i}, \\
& \dot{\psi}=\omega_{0},
\end{aligned}
$$


где $\mu=I^{-1}, \quad \gamma-\omega_{0}=\mu \Delta-$ частотная расстройка (зона главного резонанса), $\eta_{i}=\varphi_{i}-\psi-$ фазовые расстройки.

Далее предполагаем, что параметры индивидуального ротатора выбираются из области (2) или (3) на рис. 1.1, для которых в фазовом пространстве имеется вращательный предельный цикл. Считаем, что $\mu \square 1$.

Усредняя систему (5.17) по быстрой фазе и далее преобразуя время $\mu \tau=\tau_{n}$, получаем укороченную систему вида

$$
\begin{aligned}
& \dot{\eta}_{i}=\xi_{i}, \\
& \dot{\xi}_{i}=-\xi_{i}-\frac{A}{2 \omega_{0}^{2}} \sin \eta_{i}+\Delta+\sigma\left(\xi_{i-1}-2 \xi_{i}+\xi_{i+1}\right)
\end{aligned}
$$

с граничными условиями $\xi_{0}=\xi_{1}, \xi_{N}=\xi_{N+1}$.

В свою очередь (5.18) заменой времени $\sqrt{\frac{A}{2 \omega_{0}^{2}}} \tau_{n}=\tau_{n n}$ сводится к уравнениям вида

$$
\begin{aligned}
& \ddot{\eta}_{i}+\lambda_{1}^{r} \dot{\eta}_{i}+\sin \eta_{i}=\gamma_{1}^{r}+\sigma_{0}\left(\dot{\eta}_{i-1}-2 \dot{\eta}_{i}+\dot{\eta}_{i+1}\right), \\
& i=\overline{1, N}
\end{aligned}
$$

с граничными условиями $\eta_{0} \equiv \eta_{1}, \eta_{N} \equiv \eta_{N+1}$. Здесь $\lambda_{1}^{r}=\sqrt{\frac{2 \omega_{0}^{2}}{A}}$, $\gamma_{1}^{r}=\frac{2 \omega_{0}^{2} \Delta}{A}, \sigma_{0}=\sqrt{\frac{2 \omega_{0}^{2}}{A}} \sigma$.

Как видим, усредненная система (5.19) имеет тот же вид, что и система уравнений автономной цепочки. Это значит, что характер пространственно однородного динамического режима цепочки определяется динамикой «усредненного» ротатора:

$$
\ddot{\eta}+\lambda_{1}^{r} \dot{\eta}+\sin \eta=\gamma_{1}^{r} \text {. }
$$

Бифуркационная диаграмма этого уравнения на плоскости параметров $\left(\gamma_{1}^{r}, \lambda_{1}^{r}\right)$ с точностью до обозначения осей совпадает с картиной на рис. 1.1. Для ее интерпретации на исходную неавто- 
номную систему (5.17) нужно сделать единственную поправку заменить кривую Трикоми на узкую полоску, соответствующую хаотическим тор-аттракторам системы (5.17) (см. разд. 1.2). Напомним, что из принципа усреднения следует: если $L$ - некоторое предельное множество траекторий усредненной автономной системы, то $L \times S^{1}$ - соответствующее предельное множество неавтономной системы вместе с условиями устойчивости $L$.

Таким образом, имеется все необходимое для описания динамики цепочки в рассматриваемой области параметров.

Для параметров из области (1) в цепочке неавтономных ротаторов (5.12) будет наблюдаться пространственно однородный режим синхронных вращений (взаимно принудительная синхронизация), реализующийся при любых начальных условиях системы. Для области (3) - режим квазипериодических вращений маятников, когда имеет место их взаимная синхронизация, но отсутствует таковая с внешним источником (режим биений). Для области (2) могут реализоваться тот или другой режимы в зависимости от начальных условий. По доказанному выше свойству автономной цепочки перечисленные динамические режимы устойчивы при любом, в том числе и сколь угодно малом, значении параметра связи $\sigma_{0}$. Для параметров вблизи кривой Трикоми будет наблюдаться режим синхронных, но вместе с тем хаотических вращений маятников хаотическая синхронизация [68]. В отличие от предыдущих случаев устойчивость этого динамического режима реализуется при значениях связи не меньшей пороговой величины, которая зависит как от ляпуновских показателей аттракторов, так и от числа ротаторов в цепочке (кольце) (см. раздел 5.1).

\section{3. Регулярная и хаотическая синхронизация в однородной цепочке динамических систем ротатор - осциллятор}

Динамика системы ротатор - осциллятор исследована в разделе 3.2. В частности, установлено существование в этой системе хаотических аттракторов лоренцевского типа, аттрактора Фейгенбаума и перемежаемости хаосов. Ниже рассмотрим динамику цепочки диффузионно-связанных систем данного типа [69, 70] - синхронизацию вращательных движений ротаторов. 
Физический пример. На рис. 5.4 изображена цепочка сверхпроводящих переходов, каждый из которых нагружен колебательным контуром. Считаем все переходы, а также соответствующие элементы электрических схем идентичными.

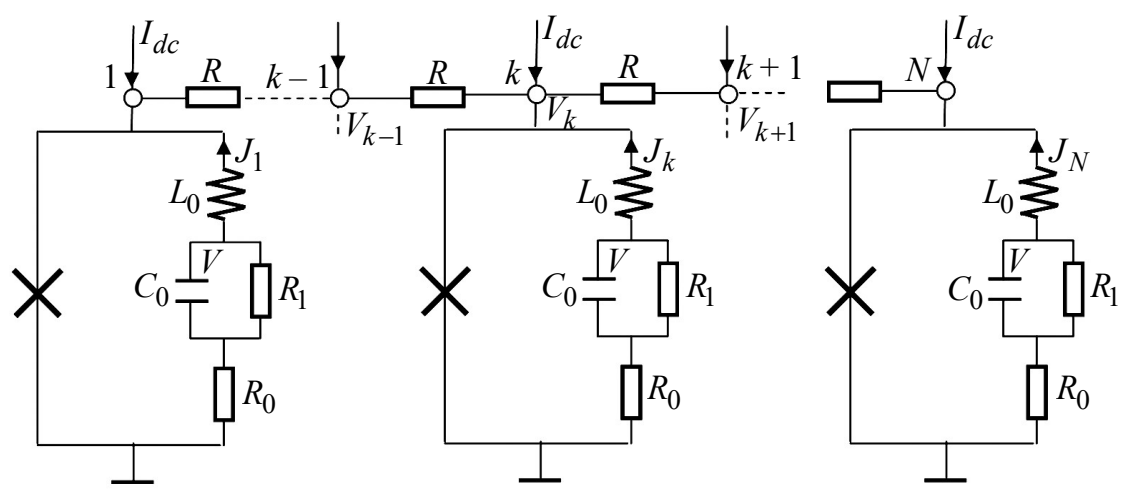

Рис. 5.4. Цепочка со сверхпроводящими переходами.

Используя законы Кирхгоффа (для указанных на рис. 5.4 узлов схемы) и соотношение Джозефсона, проводя далее процедуру обезразмеривания физических переменных, получаем динамическую систему цепочки в следующем виде:

$$
\begin{aligned}
& c \ddot{\varphi}_{k}+\dot{\varphi}_{k}+\sin \varphi_{k}=\gamma+\varepsilon\left(\dot{\varphi}_{k-1}-2 \dot{\varphi}_{k}+\dot{\varphi}_{k+1}\right)+J_{k}, \\
& \ddot{J}_{k}+\delta \dot{J}_{k}+\omega_{0}^{2} J_{k}=b \ddot{\varphi}_{k}+d \dot{\varphi}_{k}, \\
& k=\overline{1, N}
\end{aligned}
$$

с граничными условиями $\varphi_{0} \equiv \varphi_{1}, \varphi_{N} \equiv \varphi_{N+1}$.

Связь параметров системы (5.20) с безразмерными параметрами схемы осуществляется по формулам:

$$
\delta=\frac{c_{0} r_{0} r_{1}+l_{0}}{c_{0} l_{0} r_{1}}, \omega_{0}^{2}=\frac{1}{c_{0} l_{0}}\left(1+\frac{r_{0}}{r_{1}}\right), b=-\frac{1}{l_{0}}, d=-\frac{1}{c_{0} l_{0} r_{1}}, \varepsilon=\frac{1}{r} .
$$


Исследование синхронизации проводим в разработанной выше технике усреднения. Рассматриваем область параметров

$$
D_{\mu}=\left\{c^{-1}=\mu \ll 1, \delta=\mu h, \gamma+\frac{d}{\omega_{0}}-\omega_{0}=\mu \Delta\right\},
$$

соответствующую резонансной зоне частот ротаторов и осцилляторов.

Нетрудно убедиться, что уравнения (5.20) эквивалентны следующей системе:

$$
\begin{aligned}
& c \ddot{\varphi}_{k}+\dot{\varphi}_{k}+\sin \varphi_{k}=\gamma+J_{k}+\varepsilon\left(\dot{\varphi}_{k-1}-2 \dot{\varphi}_{k}+\dot{\varphi}_{k+1}\right), \\
& \dot{J}_{k}=W_{k}+b \dot{\varphi}_{k}-\mu h J_{k}, \\
& \dot{W}_{k}=-\omega_{0}^{2} J_{k}+d \dot{\varphi}_{k} .
\end{aligned}
$$

В свою очередь уравнения (5.21) заменой переменных

$$
\begin{aligned}
J_{k} & =\theta_{k} \sin \varphi_{k}+\eta_{k} \cos \varphi_{k}+\frac{d}{\omega_{0}}, \\
W_{k} & =\left(\theta_{k} \cos \varphi_{k}-\eta_{k} \sin \varphi_{k}\right) \omega_{0}-b \omega_{0}, \\
\dot{\varphi}_{k} & =\omega_{0}+\mu \Phi_{k}\left(\theta_{k}, \eta_{k}, \varphi_{k}, \xi_{k}\right), \\
\eta_{k} & =\varphi_{k}-\varphi_{1},
\end{aligned}
$$

где $\Phi_{k}=\frac{1-\theta_{k}}{\omega_{0}} \cos \varphi_{k}+\frac{\eta_{k}}{\omega_{0}} \sin \varphi_{k}+\xi_{k}$, сводятся к эквивалентной системе в стандартной форме вида

$$
\begin{aligned}
& \dot{\theta}_{k}=\mu \Theta_{k}\left(\theta_{k}, \eta_{k}, \varphi_{k}, \xi_{k}\right), \\
& \dot{\eta}_{k}=\mu T_{k}\left(\theta_{k}, \eta_{k}, \varphi_{k}, \xi_{k}\right), \\
& \dot{\xi}_{k}=\mu \Xi_{k}\left(\theta_{k}, \eta_{k}, \varphi_{k}, \xi_{k-1}, \xi_{k}, \xi_{k+1}\right), \\
& \dot{\psi}_{k}=\mu\left(\Phi_{k}-\Phi_{1}\right), \\
& \dot{\varphi}_{1}=\omega_{0}+\mu \Phi_{1}\left(\theta_{1}, \eta_{1}, \varphi_{1}, \xi_{1}\right)
\end{aligned}
$$

с функциями правых частей: 


$$
\begin{gathered}
\Theta_{k}=\left(\eta_{k}+b \sin \varphi_{k}+\frac{d}{\omega_{0}} \cos \varphi_{k}\right) \Phi_{k}- \\
-h\left(\theta_{k} \sin \varphi_{k}+\eta_{k} \cos \varphi_{k}\right) \sin \varphi_{k}+\frac{d}{\omega_{0}} \sin \varphi_{k} \\
T_{k}=\left(b \cos \varphi_{k}-\theta_{k}-\frac{d}{\omega_{0}} \sin \varphi_{k}\right) \Phi_{k}- \\
-h\left(\theta_{k} \sin \varphi_{k}+\eta_{k} \cos \varphi_{k}\right) \cos \varphi_{k}+\frac{d}{\omega_{0}} \cos \varphi_{k}, \\
\Xi_{k}=\Delta-\left(\frac{\partial \Phi_{k}}{\partial \theta_{k}} \Theta_{k}+\frac{\partial \Phi_{k}}{\partial \eta_{k}} \mathrm{~T}_{k}+\frac{\partial \Phi_{k}}{\partial \varphi_{k}} \Phi_{k}+\Phi_{k}\right)+\varepsilon\left(\Phi_{k-1}-2 \Phi_{k}+\Phi_{k+1}\right)
\end{gathered}
$$

Усредненная система для (5.22) имеет следующий вид:

$$
\begin{aligned}
& \dot{\xi}_{k}=\mu\left(-b_{1} \xi_{k}+b_{2} \theta_{k}+b_{3} \eta_{k}+\Delta+\beta\left(\xi_{k-1}-2 \xi_{k}+\xi_{k+1}\right)\right), \\
& \dot{\theta}_{k}=\mu\left(-b_{4} \theta_{k}+b_{5} \eta_{k}+\eta_{k} \xi_{k}+b_{6}\right), \\
& \dot{\eta}_{k}=\mu\left(-b_{4} \eta_{k}-b_{5} \theta_{k}-\theta_{k} \xi_{k}+b_{7}\right), \\
& \dot{\psi}_{k}=\mu\left(\xi_{k}-\xi_{1}\right), \\
& \dot{\varphi}_{1}=\omega_{0}+\mu \xi_{1}
\end{aligned}
$$

с граничными условиями $\xi_{0} \equiv \xi_{1}, \xi_{N} \equiv \xi_{N+1}$ и выражениями для параметров

$$
\begin{gathered}
b_{1}=1-\frac{d}{\omega_{0}^{2}}, b_{2}=0, b_{3}=\frac{1}{2 \omega_{0}^{2}}, b_{4}=\frac{1}{2}\left(h+\frac{d}{\omega_{0}^{2}}\right), b_{5}=\frac{b}{2 \omega_{0}}, \\
b_{6}=\frac{d}{2 \omega_{0}^{2}}, b_{7}=\frac{b}{2 \omega_{0}} .
\end{gathered}
$$

Замена переменных

$$
\begin{aligned}
& x_{k}=b_{4}^{-1}\left(\xi_{k}+b_{5}\right), \quad y_{k}=\left(b_{3} \eta_{k}+b_{2} \theta_{k}\right) / b_{1} b_{4}-\Lambda, \\
& z_{k}=\left(b_{3} \theta_{k}-b_{2} \eta_{k}\right) / b_{1} b_{4}+R, \quad \mu b_{4} \tau=\tau_{\mathrm{н}}
\end{aligned}
$$

сводит первые $3 N$ уравнений укороченной системы (5.23) к цепочке диффузионно-связанных систем лоренцевского типа: 


$$
\begin{aligned}
& \dot{x}_{k}=-\sigma\left(x_{k}-y_{k}\right)+\rho+\varepsilon\left(x_{k-1}-2 x_{k}+x_{k+1}\right), \\
& \dot{y}_{k}=-y_{k}+R x_{k}-x_{k} z_{k}, \\
& \dot{z}_{k}=-z_{k}+x_{k} y_{k}+\Lambda x_{k}, \\
& k=\overline{1, N}
\end{aligned}
$$

с граничными условиями $x_{0} \equiv x_{1}, x_{N} \equiv x_{N+1}$. Параметры имеют выражения:

$$
\begin{gathered}
\sigma=b_{1} / b_{4}, \quad R=\frac{b_{2} b_{7}-b_{3} b_{6}}{b_{1} b_{4}^{2}}, \Lambda=\frac{b_{3} b_{7}+b_{2} b_{6}}{b_{1} b_{4}^{2}}, \\
\rho=\left(\Delta+\left(b_{1} b_{4} b_{5}+b_{3} b_{7}+b_{2} b_{6}\right) b_{4}^{-1}\right) b_{4}^{-2}, \quad \varepsilon=\frac{\beta}{b_{4}} .
\end{gathered}
$$

Далее будем исследовать изохронную синхронизацию в цепочке (5.24) и результаты интерпретировать на исходную систему (5.20).

Динамические свойства парциальной системы в (5.24) $(\varepsilon=0)$ исследованы в разделе 3.2. В частности, при помощи квадратичной формы

$$
V_{k}=\frac{1}{2}\left(x_{k}^{2}+\left(y_{k}+\Lambda\right)^{2}+\left(z_{k}-\sigma-R\right)^{2}\right)
$$

установлен шар ее диссипации. Производная этой квадратичной формы вдоль векторного поля парциальной системы имеет вид

$$
\dot{V}_{k}=-\sigma x_{k}^{2}-(\rho-\sigma \Lambda) x_{k}-y_{k}^{2}-\Lambda y_{k}-z_{k}^{2}+(\sigma+R) z_{k} .
$$

Из правой части этого уравнения следует, что найдется такой шар $U_{k}: x_{k}^{2}+\left(y_{k}+\Lambda\right)^{2}+\left(z_{k}-\sigma-R\right)^{2} \leq L^{2}$, вне которого производная квадратичной формы $\dot{V}_{k}<0$, где $L-$ постоянная, зависящая от параметров $\sigma, R, \Lambda$ и $\rho$. На основе этого свойства парциальной системы сформулируем свойство диссипативности связанной системы (5.24) в виде следующей леммы.

Лемма 5.2. Система (5.24) диссипативна в шар $U=\bigcup_{k=1}^{N} U_{k}$. 
Доказательство. Рассматриваем квадратичную форму $V=\sum_{k=1}^{N} V_{k}$. Ее производная в силу системы (5.24) имеет вид и оценку

$$
\dot{V}=\sum_{k=1}^{N} \dot{V}_{k}-\varepsilon \sum_{k=1}^{N}\left(x_{k-1}-x_{k}\right)^{2} \leq \sum_{k=1}^{N} \dot{V}_{k} .
$$

Поскольку каждое из слагаемых последней суммы отрицательно вне шара $U_{k}$, то $\dot{V} \leq 0$ вне $U=\bigcup_{k=1}^{N} U_{k}$ - шара диссипации системы (5.24).

Эта лемма позволяет нам сформулировать достаточные условия глобальной асимптотической устойчивости синхронизации в системе (5.24) в виде следующей теоремы.

Теорема 5.2. Если $\varepsilon>\alpha / 2$, где

$$
\alpha=\sup _{\forall\left(x_{k}, y_{k}, z_{k}\right) \in V_{k}}\left(\frac{\left(\sigma+R-z_{k}\right)^{2}+\left(y_{k}+\Lambda\right)^{2}}{4}-\sigma\right),
$$

то интегральное многообразие $M_{0}=\left(x_{k}=x_{k+1}, y_{k}=y_{k+1}\right.$, $\left.z_{k}=z_{k+1}\right), \quad k=\overline{1, N} \quad$ в цепочке (5.24) с числом осцилляторов $N \leq N_{0}=[\pi / \arccos v]$, где $v=(2 \varepsilon-\alpha) / 2 \varepsilon$, глобально асимптотически устойчиво.

Доказательство. Запишем систему (5.24) относительно переменных $u_{k}=x_{k}-x_{k+1}, v_{k}=y_{k}-y_{k+1}, \quad w_{k}=z_{k}-z_{k+1}$ - трансверсалей многообразия $M_{0}$ :

$$
\begin{aligned}
& \dot{u}_{k}=-\sigma\left(u_{k}-v_{k}\right)+\varepsilon\left(u_{k-1}-2 u_{k}+u_{k+1}\right), \\
& \dot{v}_{k}=-v_{k}+\left(R-z_{k}\right) u_{k}-x_{k} w_{k}+u_{k} w_{k}, \\
& \dot{w}_{k}=-w_{k}+\left(y_{k}+\Lambda\right) u_{k}+x_{k} v_{k}-u_{k} v_{k}, \\
& k=\overline{1, N-1}, u_{0} \equiv 0 .
\end{aligned}
$$


Рассматриваем функцию Ляпунова $V=\frac{1}{2} \sum_{k=1}^{N-1} V_{k}, V_{k}=u_{k}^{2}+v_{k}^{2}+w_{k}^{2}$. Ее производная в силу системы (5.25) имеет вид

$$
\begin{gathered}
\dot{V}=-\sum_{k=1}^{N-1}\left((\sigma+\alpha) u_{k}^{2}+v_{k}^{2}+w_{k}^{2}+\left(\sigma+R-z_{k}\right) u_{k} v_{k}+\left(y_{k}+\Lambda\right) u_{k} w_{k}\right)-\varepsilon \Phi, \\
\Phi=\sum_{k=1}^{N-1}\left(2 v u_{k}^{2}-2 u_{k} u_{k+1}\right),
\end{gathered}
$$

где $v=1-\alpha / 2 \varepsilon, \alpha-$ параметр, подлежащий выбору.

По лемме все фазовые траектории (5.24) попадают в шар $U=\bigcup_{k=1}^{N} U_{k}$ и не покидают его при $t \rightarrow \infty$. Выберем значение параметра $\alpha$ следующим образом: $\alpha=\sup _{\forall\left(x_{k}, y_{k}, z_{k}\right) \in V_{k}}\left(\frac{\left(\sigma+R-z_{k}\right)^{2}+\left(y_{k}+\Lambda\right)^{2}}{4}-\sigma\right)$. При этом каждая из квадратичных форм под знаком первой суммы положительна. Остается определить условия положительности квадратичной формы $\Phi=\sum_{k=1}^{N-1}\left(2 v U_{k}^{2}-2 U_{k} U_{k+1}\right)$ в уравнении (5.26).

Можно убедиться, что значения главных миноров $\Delta_{k}$ матрицы квадратичной формы $Ф$

$$
\left(\begin{array}{cccccccccc}
2 v & -1 & 0 & 0 & & & & & \\
-1 & 2 v & -1 & 0 & & & & & \\
0 & -1 & 2 v & -1 & & & & & \\
0 & 0 & -1 & 2 v & & & & & \\
& 0 & & \ddots & & 0 & & \\
& & & & & 2 v & -1 & 0 & 0 \\
& & & & & -1 & 2 v & -1 & 0 \\
& & & & & 0 & -1 & 2 v & -1 \\
& & & & & 0 & 0 & -1 & 2 v
\end{array}\right)
$$


являются решениями рекуррентного уравнения

$$
\Delta_{k}=2 v \Delta_{k-1}-\Delta_{k-2}, \Delta_{-1}=0, \Delta_{0}=1, k=\overline{1, N-1} .
$$

Поскольку $\varepsilon>\alpha / 2$, то $0<v<1$, и в этом случае данное уравнение имеет решение:

$$
\Delta_{k}=\frac{\sin (k+1) \theta}{\sin \theta}, \text { где } \theta=\arccos v .
$$

После несложных вычислений получаем, что при всех $N \leq N_{0}=[\pi / \arccos v]$ значения $\Delta_{k}>0, k=\overline{1, N-1}$. То есть в условиях теоремы квадратичная форма Ф положительна во всем фазовом пространстве, а значит, и тривиальное решение (5.25), соответствующее изохронной синхронизации в системах (5.24) и (5.20), является ГАУ.

Таким образом, для параметров уравнений (5.20), определенных условиями теоремы, в цепочке будет наблюдаться режим синхронизации вращений ротаторов, реализующийся при любых начальных условиях системы. При этом характер синхронных вращений будет полностью определяться параметрами и динамическими режимами одиночной системы ротатор - осииллятор, изученными в разделе 3.2. Изменяя некоторый параметр системы (например, $R)$, можно последовательно наблюдать режимы периодической, квазипериодической и хаотической взаимной синхронизации вращений ротаторов. При наблюдении динамики отдельных элементов цепочки (парциальных систем) в их фазовых пространствах обнаруживается (или обнаружилась бы) полная идентичность фазовых портретов (см. раздел 3.2) с синхронным движением парциальных изображающих точек по траекториям этих портретов. Без комментариев обратим внимание на то, что условия локальной устойчивости $\varepsilon>\varepsilon^{*} / 4 \sin ^{2} \frac{\pi}{2 N}$ синхронизации и условия ее глобальной устойчивости $\varepsilon>\alpha / 4 \sin ^{2} \frac{\pi}{2 N}$ совпадают по форме. 


\section{Глава 6 \\ ФИЗИКА, СУЩЕСТВОВАНИЕ, СИНТЕЗ И УСТОЙЧИВОСТЬ КЛАСТЕРНЫХ СТРУКТУР}

Кластером в решетках динамических систем называется группа элементов решетки, обладающих идентичным динамическим поведением. Динамические системы, принадлежащие одному и тому же кластеру, могут как соседствовать, так и не соседствовать друг с другом, распределяясь по целочисленным координатам решетки некоторым образом. При этом, если динамическое состояние решетки характеризуется двумя и более кластерами, то распределение их элементов по координатам решетки образует кластерную структуру (КС). В решетках правильной геометрической формы (цепочка, кольцо, двумерная прямоугольная решетка и др.) такие структуры, выполненные в цвете, имеют вид разноцветных (по количеству кластеров) бордюров и паркетов, согласованных с различными типами симметрий этих решеток. Заметим, что в калейдоскопе всевозможных структур изученная выше пространственно однородная структура, определяемая изохронной синхронизацией всех осцилляторов решетки, является самой простой структурой (все осцилляторы решетки образуют единый кластер - одноцветная КС).

Представляющие интерес решетки имеют в качестве элементов автоколебательные системы, поэтому можно утверждать, что, как и в случае пространственно однородной структуры, однотипность динамики кластеров определяется процессами синхронизации. Другое дело, что эта синхронизация проявляет себя достаточно необычным образом. В данном контексте возникают естественные вопросы о природе «необычных» субъектов синхронизации, образующихся в решетке в порядке самоорганизации и определяющих нетривиальные кластерные структуры (это уже не одиночные осцилляторы). Параллельно возникают вопросы о роли геометрических параметров решеток, влиянии различного типа симметрий решеток на число и вид всевозможных КС, а также вопросы, связанные с устойчивостью кластерных структур. Ответам на эти и другие вопросы, связанные с кластерными структурами, посвящена данная глава. Далее мы будем использовать иллюстрацион- 
ный материал из динамики осцилляторов (они более удобны для численного эксперимента и демонстрации), но все сказанное будет в полной мере относиться и к главному предмету нашего интереса динамике ротаторов.

\section{1. Физика кластерных структур}

Представим существующую интерпретацию кластерных структур [71-78] в однородных решетках на примере цепочки осцилляторов, уравнения которой для удобства перепишем:

$$
\begin{aligned}
& \dot{\mathbf{X}}_{i}=\mathbf{F}\left(\mathbf{X}_{i}, t\right)+\varepsilon \mathbf{C}\left(X_{i-1}-2 X_{i}+X_{i+1}\right), \\
& i=\overline{1, N}, \quad \mathbf{X}_{0} \equiv \mathbf{X}_{1}, \quad \mathbf{X}_{N} \equiv \mathbf{X}_{N+1} .
\end{aligned}
$$

Как указывалось, система (6.1) имеет интегральное многообразие $M_{0}=\left\{\mathbf{X}_{i}=\mathbf{X}_{i+1}, i=\overline{1, N}\right\}$. Его существование очевидно, и оно сыграло свою роль в исследовании синхронизации осцилляторов (ротаторов), порождавшей пространственно однородное состояние решетки. Оказывается, однако, что кроме $M_{0}$ в цепочке существуют и другие («кластерные») инвариантные многообразия, которые имеют следующий вид [75]:

$$
\begin{aligned}
& M_{N, n+1}=\left\{\mathbf{X}_{2 n+1}=\mathbf{X}_{1}, \mathbf{X}_{2 n}=\mathbf{X}_{2}, . ., \mathbf{X}_{n+2}=\mathbf{X}_{n}\right\}, \text { если } N=2 n+1 ; \\
& M_{N, n}=\left\{\mathbf{X}_{2 n}=\mathbf{X}_{1}, \mathbf{X}_{2 n-1}=\mathbf{X}_{2}, . ., \mathbf{X}_{n+1}=\mathbf{X}_{n}\right\}, \text { если } N=2 n ; \\
& M_{p q, q}=\left\{\mathbf{X}_{i}=\mathbf{X}_{i+2 q j}, j=1,2, . .,[(p-1) / 2], i=\overline{1, q}\right\}, \\
& M_{p q, q}=\left\{\mathbf{X}_{i}=\mathbf{X}_{-i+1+2 q j}, j=1,2, . .,[p / 2], i=\overline{1, q}\right\}, \text { если } N=p q,
\end{aligned}
$$

где $p, q$ - целые числа.

Если допустить, что некоторое из представленных многообразий устойчиво, то его уравнения и представляют аналитическое выражение для соответствующей кластерной структуры. В частности, если $N=6$, то таких многообразий два:

$$
\begin{aligned}
& M_{6,3}=\left\{\mathbf{X}_{6}=\mathbf{X}_{1}, \mathbf{X}_{5}=\mathbf{X}_{2}, \mathbf{X}_{4}=\mathbf{X}_{3}\right\} \text { и } \\
& M_{6,2}=\left\{\mathbf{X}_{1}=\mathbf{X}_{4}=\mathbf{X}_{5}, \mathbf{X}_{2}=\mathbf{X}_{3}=\mathbf{X}_{6}\right\} .
\end{aligned}
$$


В зависимости от начальных условий в цепочке будет наблюдаться одна из кластерных структур, схематично изображенных на рис. 6.1. На данном рисунке одинаково окрашенные квадраты соответствуют осцилляторам цепочки с идентичной динамикой. Далее подобные изображения будем называть схемами кластерных структур.
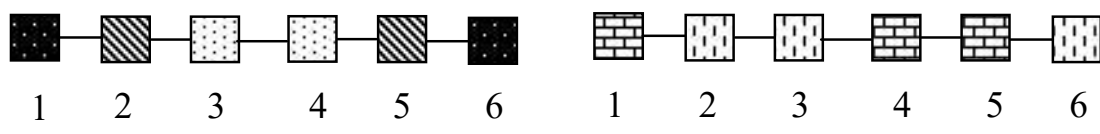

a

6

Рис. 6.1. Кластерные структуры в цепочке из $N=6$ осцилляторов.

Динамика решеток на многообразиях получила название «cluster synchronization» (кластерная синхронизация) и исследовалась в работах [73, 76-81].

Ниже мы сформулируем кластерную динамику решеток на общедоступном языке классической синхронизации [82-84], без апелляции к их кластерным многообразиям, а последние будем получать (при необходимости) в качестве следствий.

Субъекты кластерных структур. Наблюдения кластерных структур в решетках различной геометрической размерности и формы приводят к простому заключению о субъектах кластерной синхронизации как «обобщенных» или, другими словами, групповых осцилляторах, самоорганизующихся в виде отдельных и неделимых объектов, составленных из элементарных осцилляторов. Ниже к этому заключению мы придем индуктивным путем, рассматривая динамику систем осцилляторов с простых примеров.

Рассмотрим систему двух идентичных связанных осцилляторов вида

$$
\begin{aligned}
& \dot{\mathbf{X}}_{1}=\mathbf{F}\left(\mathbf{X}_{1}\right)+\varepsilon \mathbf{C}\left(\mathbf{X}_{2}-\mathbf{X}_{1}\right), \\
& \dot{\mathbf{X}}_{2}=\mathbf{F}\left(\mathbf{X}_{2}\right)+\varepsilon \mathbf{C}\left(\mathbf{X}_{1}-\mathbf{X}_{2}\right),
\end{aligned}
$$

где $\mathbf{C}$ - матрица, определяющая структуру связи элементарных осцилляторов, $\varepsilon$ - скалярный параметр. Очевидно, что в обычном случае все стационарные динамические режимы этой системы можно подразделить на два типа: 
1) режим изохронной регулярной или хаотической взаимной синхронизации. Этому режиму соответствует решение $\mathbf{X}_{1}(t)=$ $=\mathbf{X}_{2}(t)=\mathbf{X}(t)$. Характер синхронных движений осцилляторов определяется параметрами и типом аттрактора (регулярный, хаотический) одиночного осциллятора $A(1)$. Важно: в этом случае связанная система (6.2) распадается на пару синхронизованных осцилляторов;

2) режим неизохронной динамики, соответствующий аттрактоpy $A_{s}(2)$. Этот режим может представлять собой или стационарные биения (регулярные или хаотические), или неизохронную синхронизацию. При движении системы на аттракторе $A_{s}(2)$ $\mathbf{X}_{1}(t) \neq \mathbf{X}_{2}(t)$. Заметим, что в этом случае система (6.2) представляет собой единый и неделимый в указанном смысле объект.

Для иллюстрации сказанного на рис. 6.2 представлены режим хаотической синхронизации и режим биений хаотических автогенераторов Чуа $[85,86]$. Одиночный осциллятор Чуа описывается динамической системой вида

$$
\begin{aligned}
& \dot{x}=\alpha(y-h(x)), \\
& \dot{y}=-y+z-x, \\
& \dot{z}=-\beta y-\gamma z .
\end{aligned}
$$

Здесь $h(x)=m_{1} x+\frac{m_{0}-m_{1}}{2}(|x+1|-|x-1|), \quad m_{0}<0, \alpha, \beta, \gamma, m_{1}>0 \quad-$ параметры.

Параметры эксперимента: $\left(\alpha, \beta, \gamma, m_{0}, m_{1}, \varepsilon\right)=(9,5 ; 14 ; 0,1 ;-1 / 7$; $2 / 7,1,0)$, матрица связи осцилляторов $\mathbf{C}=\operatorname{diag}(1,0,0)$.

Как видим, в первом случае фазовые портреты полностью идентичны, и это дополнительно подтверждается диагональю на фазовой плоскости одноименных переменных (третья в верхнем ряду). Это есть проекция аттрактора системы на данную плоскость. Во втором случае проекция отлична от диагонали. 

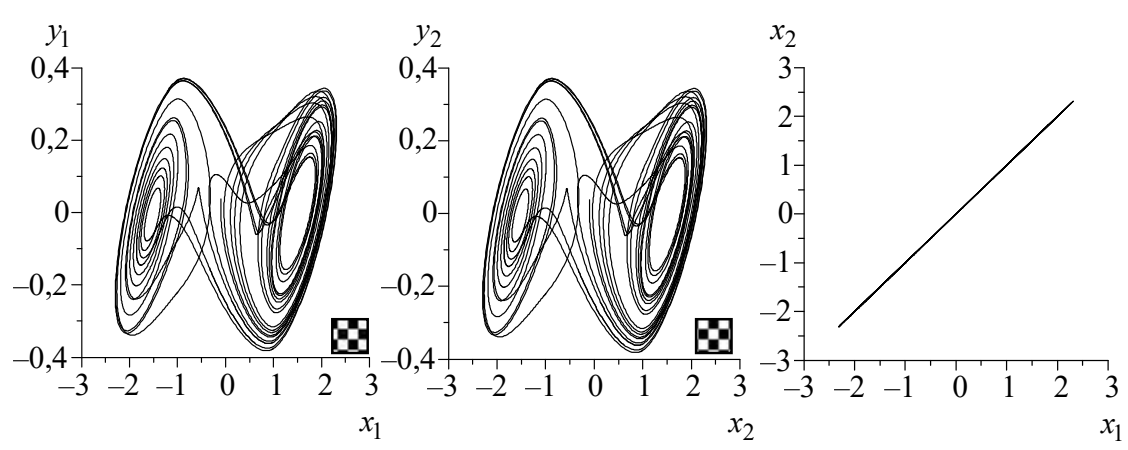

a
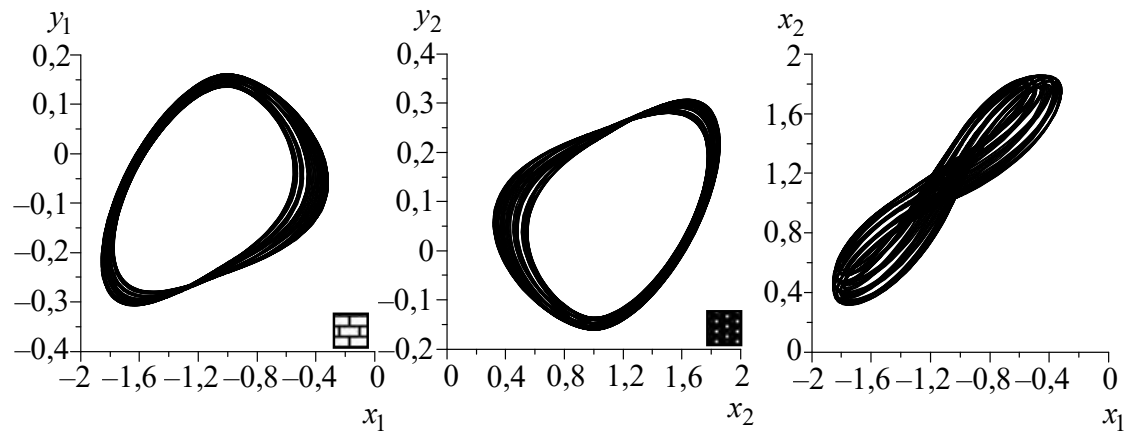

б

Рис. 6.2. Динамические режимы системы (6.2): а - аттрактор $A(1)$ режима изохронной синхронизации; б - аттрактор $A_{S}(2)$, соответствующий режиму хаотических биений, в проекциях на координатные плоскости осцилляторов

Схематично динамика системы (6.2) в указанных режимах представлена на рис. 6.3.

Как говорилось, в режиме синхронизации система (6.2) распадается на пару синхронизованных осцилляторов (по условию имеется точный синхронизм). Физически этот факт соответствует удалению связи между соответствующими осцилляторами без нарушения их динамического режима. В частности, если осцилляторы являются радиотехническими автогенераторами, то эта процедура соответствует разрезанию связей между эквипотенциальными точками - один из приемов эквивалентных преобразований электрических цепей. Заметим, что пространственно однородная структура 
разрезается на отдельные осцилляторы, а кластерная структура (см. рис. 6.1) разрезается на идентичные блоки. В дальнейшем процедура разрезания схем кластерных структур (связей между «одноцветными» осцилляторами) будет использоваться в качестве приема их эквивалентных преобразований, как и, наоборот, соединение блоков осцилляторов по соответствующим точкам (соединение «одноцветных» осцилляторов) при синтезе схем кластерных структур.

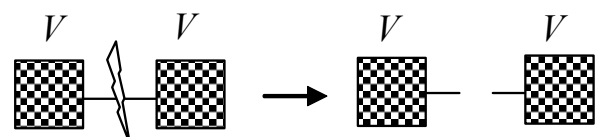

a

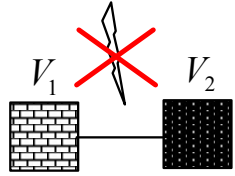

6

Рис. 6.3. Схематичное изображение режимов 1 и 2 связанной системы. Над связью осцилляторов изображен символический «нож».

Обратимся снова к системе (6.2). Допустим, что в этой системе существует аттрактор $A_{s}(2)$ и ее движения происходят на данном аттракторе. В таком случае она представляет собой неделимый объект, условно - обобщенный осциллятор, динамика которого описывается векторным уравнением (переписанной в векторном виде системой (6.2))

$$
\dot{\mathbf{X}}=\mathbf{H}(\mathbf{X})
$$

где $\mathbf{X}=\left(\mathbf{X}_{1}, \mathbf{X}_{2}\right)^{T}, \quad \mathbf{H}(\mathbf{X})=\left(\mathbf{F}\left(\mathbf{X}_{1}\right), \mathbf{F}\left(\mathbf{X}_{2}\right)\right)^{T}+\varepsilon \mathbf{B}_{s} \otimes \mathbf{C X}$, $\mathbf{B}_{s}=\left(\begin{array}{rr}-1 & 1 \\ 1 & -1\end{array}\right)$.

Будем называть (6.3) кластерообразующим осциллятором (КО) и обозначать его $O_{s}(2)$. Индекс $s$ отражает симметричность кластерной матрицы $\mathbf{B}_{s}$, а 2 - число элементарных осцилляторов в КО, продуцирующих кластеры (точное определение КО приведем ниже). Схематично КО в стационарном «рабочем» режиме представлен на рис. 6.3,б. Заметим, что векторные координаты $\mathbf{X}_{1}, \mathbf{X}_{2}$ играют такую же роль, как и скалярные координаты элементарного осциллятора. 
Допустим теперь, что у нас имеется точная динамическая копия данного КО, которая описывается векторной переменной $\mathbf{Y}=\left(\mathbf{Y}_{1}, \mathbf{Y}_{2}\right)^{T}$ (динамические процессы в оригинале и копии протекают синхронно). Ответ на вопрос, каким образом следует соединять эти два КО, чтобы не нарушить их динамические режимы, содержится в предыдущих рассуждениях о разрезании/замыкании эквипотенциальных точек, соответствующих одноименным координатам. Другими словами, связи КО в синтезируемой решетке должны иметь вид $\left(\mathbf{C}^{*} \otimes \mathbf{C}\right)(\mathbf{X}-\mathbf{Y})$ или $\left(\mathbf{C}_{*} \otimes \mathbf{C}\right)(\mathbf{X}-\mathbf{Y})$, где $\mathbf{C}-$ уже известная матрица, определяющая номера связанных скалярных переменных элементарных осцилляторов, $\mathbf{C}^{*}=\operatorname{diag}(1,0)$, $\mathbf{C}_{*}=\operatorname{diag}(0,1)-$ матрицы, определяющие номера самих связанных осцилляторов, входящих в тот и другой КО (матрицы синтеза). Первая матрица определяет соединение по первым координатам $\mathbf{X}_{1}, \mathbf{Y}_{1}$, вторая - по вторым $\mathbf{X}_{2}, \mathbf{Y}_{2}$.

С учетом сказанного рассмотрим систему двух связанных КО вида

$$
\begin{aligned}
& \dot{\mathbf{X}}=\mathbf{H}(\mathbf{X})+\varepsilon\left(\mathbf{C}_{*} \otimes \mathbf{C}\right)(\mathbf{Y}-\mathbf{X}), \\
& \dot{\mathbf{Y}}=\mathbf{H}(\mathbf{Y})+\varepsilon\left(\mathbf{C}_{*} \otimes \mathbf{C}\right)(\mathbf{X}-\mathbf{Y}) .
\end{aligned}
$$

Во-первых, заметим, что уравнения (6.2) и (6.4) не отличаются по форме. Во-вторых, если систему (6.4) записать покоординатно (относительно элементарных осцилляторов), полагая при этом, что $\mathbf{Y}_{1}=\mathbf{X}_{4}, \mathbf{Y}_{2}=\mathbf{X}_{3}$, то она представит цепочку из 4 элементов. В-третьих, данная система в случае классической синхронизации КО, выражающейся равенством $\mathbf{X}=\mathbf{Y}$, определяет кластерную структуру, изображенную на рис. 6.1,б $\left(\left(\mathbf{X}_{1}, \mathbf{X}_{2}\right)^{T}=\left(\mathbf{Y}_{1}, \mathbf{Y}_{2}\right)^{T}\right.$ или $\left.\mathbf{X}_{1}=\mathbf{X}_{4}, \mathbf{X}_{2}=\mathbf{X}_{3}\right)$, на которой удалены осцилляторы с номерами 5 и 6. Для полноты картины к системе (6.4) следует подключить еще один КО.

Допустим, что в нашем распоряжении имеется еще одна динамическая копия КО $O_{s}(2)$, описываемая вектором $\mathbf{Z}=\left(Z_{1}, Z_{2}\right)^{T}$. Соединяя этот КО известным образом со вторым КО в системе (6.4), получаем систему вида 


$$
\begin{aligned}
& \dot{\mathbf{X}}=\mathbf{H}(\mathbf{X})+\varepsilon\left(\mathbf{C}_{*} \otimes \mathbf{C}\right)(\mathbf{Y}-\mathbf{X}), \\
& \dot{\mathbf{Y}}=\mathbf{H}(\mathbf{Y})+\varepsilon\left(\mathbf{C}_{*} \otimes \mathbf{C}\right)(\mathbf{X}-\mathbf{Y})+\varepsilon\left(\mathbf{C}^{*} \otimes \mathbf{C}\right)(\mathbf{Z}-\mathbf{Y}), \\
& \dot{\mathbf{Z}}=\mathbf{H}(\mathbf{Z})+\varepsilon\left(\mathbf{C}^{*} \otimes \mathbf{C}\right)(\mathbf{Y}-\mathbf{Z}) .
\end{aligned}
$$

Относительно (6.5) можно повторить то же самое, что и для (6.4). Во-первых, полагая $\mathbf{Z}_{1}=\mathbf{X}_{5}, \mathbf{Z}_{2}=\mathbf{X}_{6}$, получаем, что эта система представляет собой переформатированную цепочку из 6 элементов. Во-вторых, классическая синхронизация этой тройки КО порождает кластерную структуру, изображенную на рис. 6.1,б.

Рассмотрим еще один пример. Пусть имеется КО типа $O_{s}(3)$, составленный из трех элементарных осцилляторов: $\mathbf{X}=$ $=\left(\mathbf{X}_{1}, \mathbf{X}_{2}, \mathbf{X}_{3}\right)^{T}$, а также его копия с вектором $\mathbf{Y}=\left(\mathbf{Y}_{1}, \mathbf{Y}_{2}, \mathbf{Y}_{3}\right)^{T}$. Уравнения $O_{s}(3)$ имеют тот же вид, что и (6.3), где $\mathbf{B}_{s}=\left(\begin{array}{rrr}-1 & 1 & 0 \\ 1 & -2 & 1 \\ 0 & 1 & -1\end{array}\right)$.

Синтезированная пара таких КО будет иметь уравнения того же вида, что и (6.4), где матрица синтеза заменится на $\mathbf{C}_{*}=\operatorname{diag}(0,0,1)$.

В режиме синхронизации этих КО получаем: $\left(\mathbf{X}_{1}, \mathbf{X}_{2}, \mathbf{X}_{3}\right)^{T}=$ $=\left(\mathbf{Y}_{1}, \mathbf{Y}_{2}, \mathbf{Y}_{3}\right)^{T}$. При условии, что $\mathbf{Y}_{3}=\mathbf{X}_{4}, \quad \mathbf{Y}_{2}=\mathbf{X}_{5}, \mathbf{Y}_{1}=\mathbf{X}_{6}$, это приводит к уравнениям $\mathbf{X}_{1}=\mathbf{X}_{6}, \mathbf{X}_{2}=\mathbf{X}_{5}, \mathbf{X}_{3}=\mathbf{X}_{4}$, т. е. имеем кластерную структуру, изображенную на рис. 6.1,a.

Для иллюстрации сказанного на рис. 6.4 показан кластерный аттрактор $A_{s}(3)$ КО $O_{s}(3)$, полученный в численном эксперименте с однородной цепочкой из трех осцилляторов Чуа, имеющих параметры $\left(\alpha, \beta, \gamma, m_{0}, m_{1}, \varepsilon\right)=(9,5 ; 14 ; 0,1 ;-1 / 7 ; 2 / 7 ; 0,37)$.

На рис. 6.5 изображена кластерная структура в цепочке из шести осцилляторов Чуа как результат классической синхронизация двух КО $O_{s}(3)$. 


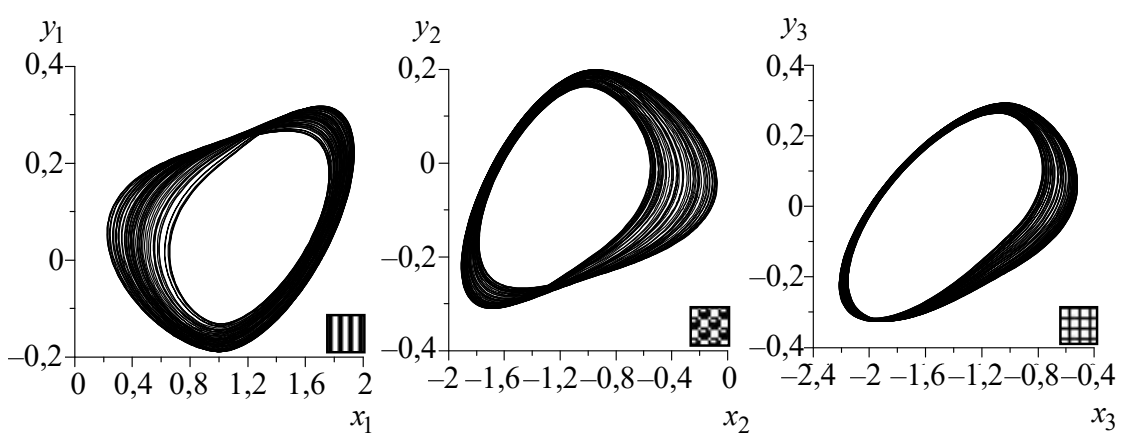

Рис. 6.4. Проекции кластерного аттрактора $A_{S}(3) \mathrm{KO} O_{S}(3)$ на координатные плоскости.
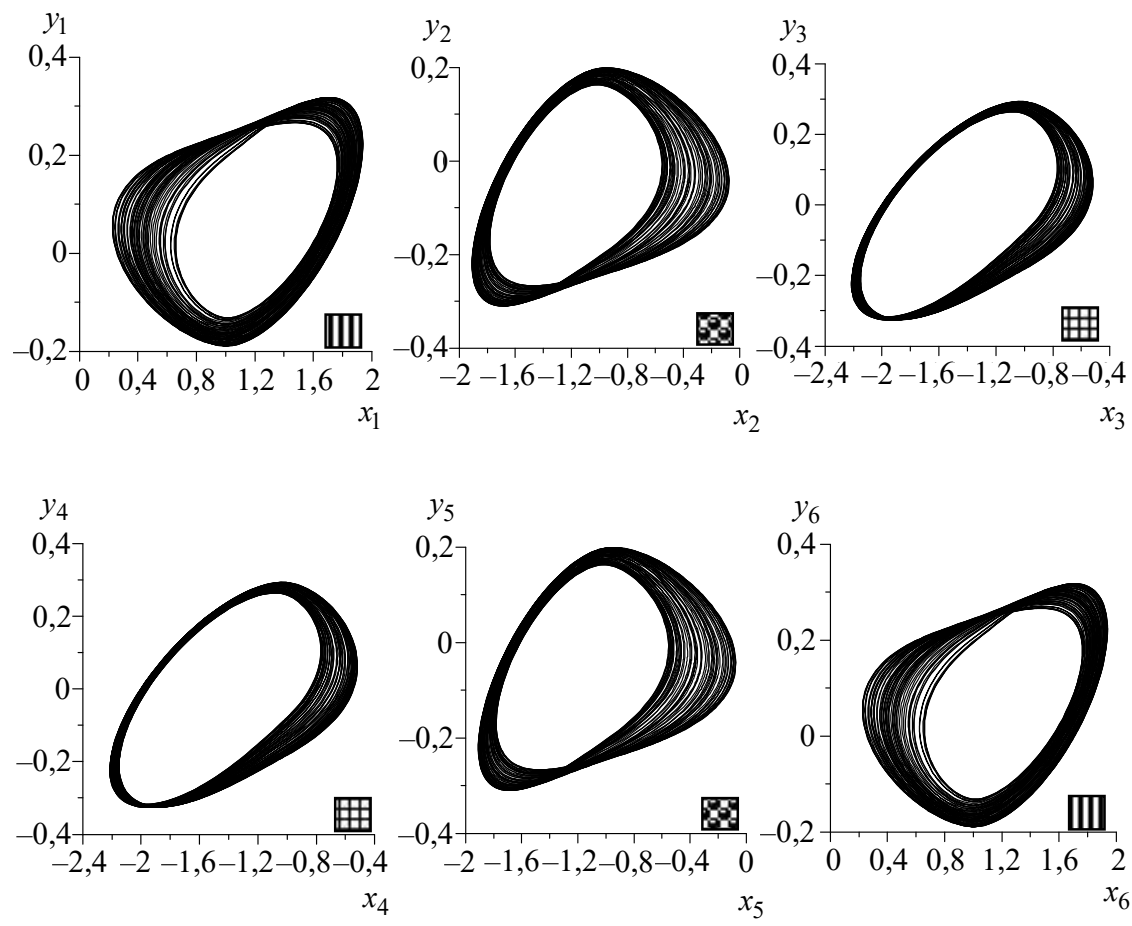

Рис. 6.5. Кластерная структура в однородной цепочке из $N=6$ осцилляторов Чуа как синхронизация двух КО $O_{s}(3)$. 
В качестве промежуточного вывода констатируем факт, что на базе КО типа $O_{s}(n)$ в цепочке из $N$ элементов может быть синтезировано столько кластерных структур, каково число делителей этого числа, и в каждом конкретном случае все эти структуры могут быть нами представлены.

Данными типами КО не исчерпываются типы кластерных структур в цепочках. В частности, если число $N$ простое, то уверенно можно сказать, что кластерная структура в такой цепочке будет относиться к другому типу. Далее будем рассматривать структуры в цепочках с нечетным числом осцилляторов $N=2 k+1$.

Рассмотрим простой случай - кластерную структуру в цепочке из трех элементарных осцилляторов, изображенную на рис. 6.6,a. Видно, что данная структура содержит два кластера и симметрична относительно оси $L$. Вопрос: каково изображение КО, определяющего эту структуру?

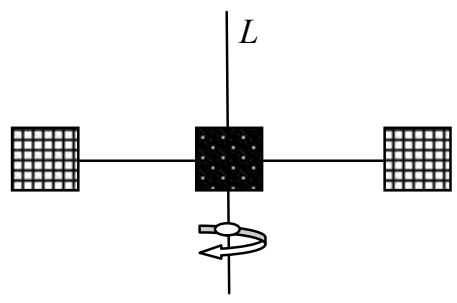

a

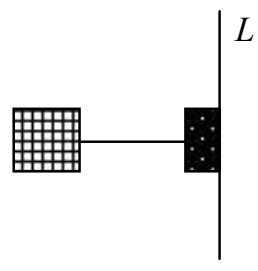

б

Рис. 6.6. Изображение кластерной структуры простой клетки (a) и кластерного осциллятора $O_{a}$ (б).

Представим себе, что данная картина изображена на двойном листе бумаги и $L$ - линия сгиба одинарных листов. Сворачивая лист, получаем картину, изображенную на рис. 6.6,б. Как видно, эта картина может претендовать на изображение КО при условии, что образовавшаяся половинка от изображения центрального элементарного осциллятора имеет физический смысл. Для выяснения этого смысла обратимся к примеру маятников Фруда, изображенных на рис. 6.7, разворачивая «историю» свертывания картины в противоположном направлении - от КО к структуре. 


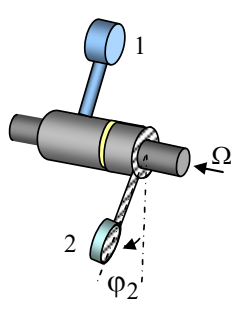

a

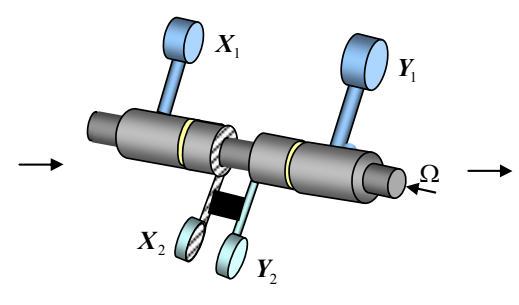

6

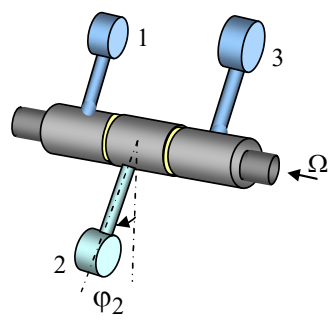

B

Рис. 6.7. Физическая интерпретация КО $O_{a}(2)$ и простой клетки на его базе.

Допустим, что имеются два соприкасающихся маятника, посаженных на вращающийся вал. Второй является «половиной» первого, «целого» маятника (рис. 6.7,a). Можно вообразить, что «половинка» получается в результате «распиливания» целого маятника в плоскости, перпендикулярной муфте и проходящей через ее середину. Допустим, что между муфтами и валом существует вязкое трение. Таковое же существует и между муфтами маятников. При этом уравнения движения системы имеют вид

$$
\begin{aligned}
& m l^{2} \ddot{\varphi}_{1}+d \dot{\varphi}_{1}+m g l \sin \varphi_{1}=R\left(\Omega-\dot{\varphi}_{1}\right)+\lambda\left(\dot{\varphi}_{2}-\dot{\varphi}_{1}\right), \\
& \frac{m}{2} l^{2} \ddot{\varphi}_{2}+\frac{d}{2} \dot{\varphi}_{2}+\frac{m}{2} g l \sin \varphi_{2}=\frac{R}{2}\left(\Omega-\dot{\varphi}_{2}\right)+\lambda\left(\dot{\varphi}_{1}-\dot{\varphi}_{2}\right) .
\end{aligned}
$$

Здесь $R\left(\Omega-\dot{\varphi}_{1}\right)$ - момент силы вязкого трения со стороны вала на первый маятник и $\frac{R}{2}\left(\Omega-\dot{\varphi}_{2}\right)-$ на второй (они пропорциональны площади соприкосновения муфты маятника с валом); $\lambda\left(\dot{\varphi}_{2}-\dot{\varphi}_{1}\right)$ и $\lambda\left(\dot{\varphi}_{1}-\dot{\varphi}_{2}\right)$ - взаимные моменты сил трения маятников; $d-$ коэффициент вязкого трения среды.

В результате введения безразмерного времени $\frac{m g l}{d+R} t=\tau$ получаем динамическую систему $\mathrm{KO} O_{a}(2)$ :

$$
\begin{aligned}
& \dot{\mathbf{X}}_{1}=\mathbf{F}\left(\mathbf{X}_{1}\right)+\varepsilon C\left(-\mathbf{X}_{1}+\mathbf{X}_{2}\right), \\
& \dot{\mathbf{X}}_{2}=\mathbf{F}\left(\mathbf{X}_{2}\right)+2 \varepsilon C\left(\mathbf{X}_{1}-\mathbf{X}_{2}\right),
\end{aligned}
$$


где $\mathbf{X}_{1}=\left(\varphi_{1}, \xi_{1}\right)^{T}, \mathbf{X}_{2}=\left(\varphi_{2}, \xi_{2}\right)^{T}, \mathbf{F}\left(\mathbf{X}_{1}\right)=\left(\gamma+I^{-1} \xi_{1},-\xi_{1}-\sin \varphi_{1}\right)^{T}$, $\mathbf{F}\left(\mathbf{X}_{2}\right)=\left(\gamma+I^{-1} \xi_{2},-\xi_{2}-\sin \varphi_{2}\right)^{T}, \quad \mathbf{C}=\operatorname{diag}(0,1), \quad I=\frac{l}{g}\left(\frac{m g l}{d+R}\right)^{2}$, $\gamma=\frac{R \Omega}{m g l}, \varepsilon=\frac{\lambda I}{d+R}$.

Как видим, в отличие от КО $O_{s}(2)(6.2)$, в котором элементарные осцилляторы связаны матрицей $\mathbf{B}_{s}=\left(\begin{array}{rr}-1 & 1 \\ 1 & -1\end{array}\right)$, в случае КО $O_{a}(2)$ (6.6) матрицей связи является асимметричная матрица $\mathbf{B}_{a}=\left(\begin{array}{cc}-1 & 1 \\ 2 & -2\end{array}\right)$. Естественно, что разница в структуре связей принципиально влияет на условия существования и характер кластерных аттракторов данных КО. Однако главное отличие $O_{s}(2)$ от $O_{a}(2)$ состоит в том, что если первый является полноценным субъектом кластерных структур, то роль второго в этом смысле ограничена. На рис. 6.7,б,в показан процесс объединения оригинала $O_{a}(2)$ с его зеркальной копией и последующим образованием кластеризованного и неделимого объекта нового типа, который далее будем называть простой клеткой (ПК). Процесс образования кластерной структуры ПК можно интерпретировать и так: после установления жесткой связи между осцилляторами-«половинками» (перемычка на рис. $6.7, б)$, в результате которой их синхронизация наступает немедленно (и по факту они становятся «целым» осциллятором), крайние осцилляторы, опосредованно взаимодействуя между собой (через центральный осциллятор), синхронизируют свои движения.

Повторим сказанное на языке уравнений. Запишем систему (6.6) в виде одного уравнения:

$$
\dot{\mathbf{X}}=\mathbf{H}(\mathbf{X}),
$$

где $\quad \mathbf{X}=\left(\mathbf{X}_{1}, \mathbf{X}_{2}\right)^{T}, \quad \mathbf{H}(\mathbf{X})=\left(\mathbf{F}\left(\mathbf{X}_{1}\right), \mathbf{F}\left(\mathbf{X}_{2}\right)\right)^{T}+\varepsilon \mathbf{B}_{a} \otimes \mathbf{C X}$, $\mathbf{B}_{a}=\left(\begin{array}{rr}-1 & 1 \\ 2 & -2\end{array}\right)$. 
Допустим, что у нас имеется точная динамическая копия данного КО, описываемая вектором $\mathbf{Y}=\left(\mathbf{Y}_{1}, \mathbf{Y}_{2}\right)^{T}$.

Рассматриваем систему этой пары КО вида

$$
\begin{aligned}
& \dot{\mathbf{X}}=\mathbf{G}(\mathbf{X})+\varepsilon \mathbf{D} \otimes C(-\mathbf{X}+\mathbf{Y}), \\
& \dot{\mathbf{Y}}=\mathbf{G}(\mathbf{Y})+\varepsilon \mathbf{D} \otimes C(\mathbf{X}-\mathbf{Y}) .
\end{aligned}
$$

Здесь $\mathbf{X}=\left(\mathbf{X}_{1}, \mathbf{X}_{2}\right)^{T}, \quad \mathbf{Y}=\left(\mathbf{Y}_{1}, \mathbf{Y}_{2}\right)^{T}, \quad \mathbf{D}=\left(\begin{array}{ll}0 & 0 \\ 1 & \lambda\end{array}\right), \quad \lambda>0-$ параметр, отвечающий за величину связи осцилляторов-«половинок». Если $\lambda$ имеет умеренные значения, то система (6.8) в целом подобна (6.4), включая порождаемую синхронизацией кластерную структуру, соответствующую решению $\mathbf{X}=\mathbf{Y}$ (см. рис. 6.1,б без 5 и 6 элементов). Однако если $\lambda \rightarrow \infty$ (устанавливается жесткая перемычка между осцилляторами-«половинками»), то, как можно показать, $\left\|\mathbf{X}_{2}-\mathbf{Y}_{2}\right\|=e^{-2 \varepsilon \lambda t} \rightarrow 0$. То есть, как и следовало ожидать, в системе (6.8) имеет место вырождение числа степеней свободы на один элементарный осциллятор («половинки» склеиваются). Полагая теперь, что $\mathbf{X}_{2} \equiv \mathbf{Y}_{2}, \quad \mathbf{Y}_{1}=\mathbf{X}_{3}$ и вводя новый вектор $\mathbf{X}$ : $\mathbf{X}=\left(\mathbf{X}_{1}, \mathbf{X}_{2}, \mathbf{X}_{3}\right)^{T}$, вместо системы (6.8) получаем систему вида

$$
\dot{\mathbf{X}}=\mathbf{S}(\mathbf{X})
$$

где $\mathbf{S}(\mathbf{X})=\left(\mathbf{F}\left(\mathbf{X}_{1}\right), \mathbf{F}\left(\mathbf{X}_{2}\right), \mathbf{F}\left(\mathbf{X}_{3}\right)\right)^{T}+\varepsilon \mathbf{B}_{s} \otimes \mathbf{C X}, \mathbf{B}_{s}=\left(\begin{array}{ccc}-1 & 1 & 0 \\ 1 & -2 & 1 \\ 0 & 1 & -1\end{array}\right)$.

Как видим, уравнение (6.9) по форме полностью совпадает с уравнением КО $O_{s}(3)$. Однако его внутреннее содержание принципиально другое. Во-первых, это уравнение рассматривается при условии существования аттрактора $A_{a}(2)$. Во-вторых, (6.6) рассматривается в определенной части фазового пространства: или в окрестности аттрактора $A_{a}(2)$ (при исследовании устойчивости структур), или на самом аттракторе, если речь идет о синтезе схем соответствующих кластерных структур. При данных условиях 
уравнение (6.9) представляет структурированный (или потенциально структурированный) (см. рис. 6.6,a), неделимый (в известном смысле) объект - простую клетку. Из сказанного выше простую клетку можно интерпретировать как предельный случай системы двух связанных КО типа $O_{a}(n)$. Приведем динамическую иллюстрацию: на рис. 6.8 изображен «рабочий» аттрактор КО $O_{a}(2)$, полученный в численном эксперименте с системой (6.6) двух осцилляторов Чуа. Параметры эксперимента: $\left(\alpha, \beta, \gamma, m_{0}, m_{1}, \varepsilon\right)=$ $=(9,5 ; 14 ; 0,1 ;-1 / 7 ; 2 / 7 ; 0,215)$, матрица $C=\operatorname{diag}(1,0,0)$.

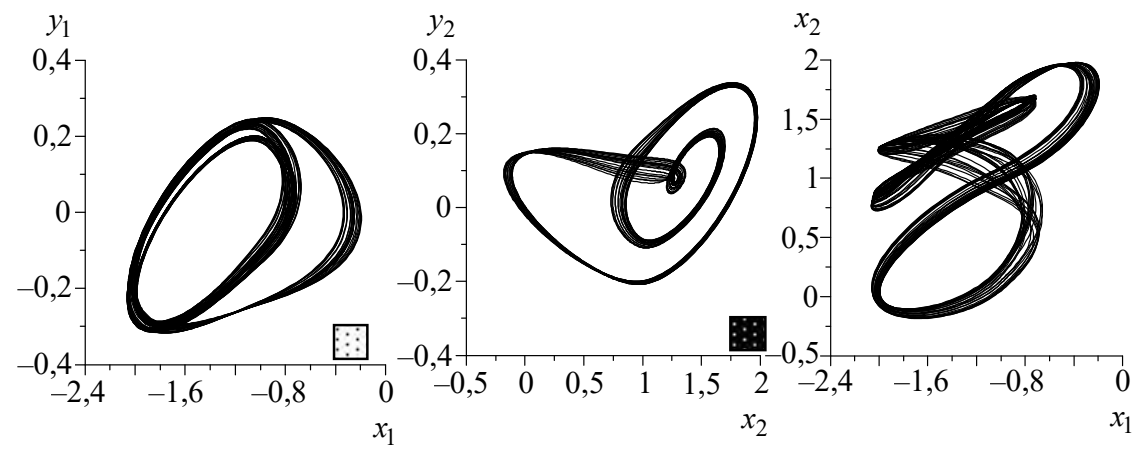

Рис. 6.8. Аттрактор $A_{a}(2)$ КО $O_{a}(2)$ в проекциях на координатные плоскости.

На рис. 6.9 представлен «рабочий» режим ПК (6.9) - динамическая кластерная структура на базе $O_{a}(2)$. Параметры системы: $\left(\alpha, \beta, \gamma, m_{0}, m_{1}, \varepsilon\right)=(9,5 ; 14 ; 0,1 ;-1 / 7 ; 2 / 7 ; 0,215), C=\operatorname{diag}(1,0,0)$.

Таким образом, простая клетка, подобно КО типа $O_{s}(n)$, также является субъектом в формировании кластерных структур в решетках. Причем, в силу симметрии собственной кластерной структуры, матрицей синтеза одномерных решеток (цепочка, кольцо) с кластерной структурой на базе простых клеток может быть любая из матриц $C_{*}$ или $C^{*}$. Проиллюстрируем сказанное на языке уравнений. 


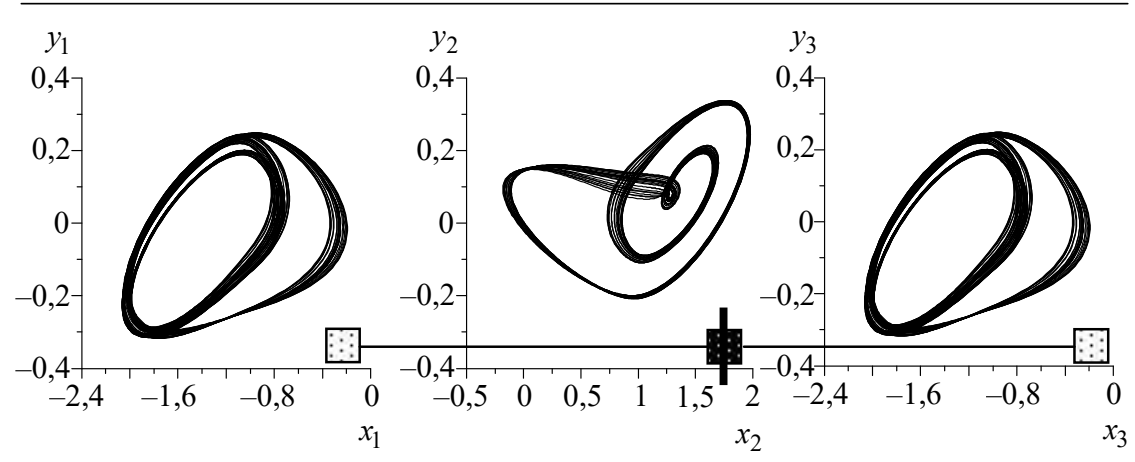

Рис. 6.9. Главный динамический режим - кластерная структура простой клетки на базе $O_{a}(2)$.

Допустим, что имеется простая клетка (6.9) с некоторой кластерной структурой и ее динамическая копия, описываемая вектором $\mathbf{Y}=\left(\mathbf{Y}_{1}, \mathbf{Y}_{2}, \mathbf{Y}_{3}\right)^{T}$. Рассмотрим систему вида

$$
\begin{aligned}
& \dot{\mathbf{X}}=\mathbf{S}(\mathbf{X})+\varepsilon\left(\mathbf{C}_{*} \otimes \mathbf{C}\right)(\mathbf{Y}-\mathbf{X}), \\
& \dot{\mathbf{Y}}=\mathbf{S}(\mathbf{Y})+\varepsilon\left(\mathbf{C}_{*} \otimes \mathbf{C}\right)(\mathbf{X}-\mathbf{Y}),
\end{aligned}
$$

где $\mathbf{C}_{*}=\operatorname{diag}(0,0,1)$.

Из условия синхронизации в (6.10) и вида собственной структуры ПК получаем $\left(\mathbf{X}_{1}, \mathbf{X}_{2}, \mathbf{X}_{3}\right)^{T}=\left(\mathbf{Y}_{1}, \mathbf{Y}_{2}, \mathbf{Y}_{3}\right)^{T}$ или $\mathbf{X}_{1}=\mathbf{X}_{3}=\mathbf{Y}_{1}=$ $=\mathbf{Y}_{3}, \mathbf{X}_{2}=\mathbf{Y}_{2}$. Переобозначая координаты вектора $\mathbf{Y}: \mathbf{Y}_{3}=\mathbf{X}_{4}$, $\mathbf{Y}_{2}=\mathbf{X}_{5}, \quad \mathbf{Y}_{1}=\mathbf{X}_{6}$, получаем координатную запись кластерной структуры в цепочке из $N=6$ элементарных осцилляторов, возникающей на базе синхронизации простых клеток: $\mathbf{X}_{1}=\mathbf{X}_{3}=\mathbf{X}_{4}=$ $=\mathbf{X}_{6}, \mathbf{X}_{2}=\mathbf{X}_{5}$. Заметим, что данная запись представляет в том числе и интегральное многообразие, имеющееся в фазовом пространстве решетки с шестью элементами (см. начало данного раздела).

На рис. 6.10 представлен результат численного эксперимента с системой (6.10), в которой элементами являются хаотические осцилляторы Чуа. Параметры эксперимента: $\left(\alpha, \beta, \gamma, m_{0}, m_{1}, \varepsilon\right)=$ $=(9,5 ; 14 ; 0,1 ;-1 / 7 ; 2 / 7 ; 0,215)$, матрица $C=\operatorname{diag}(1,0,0)$. 

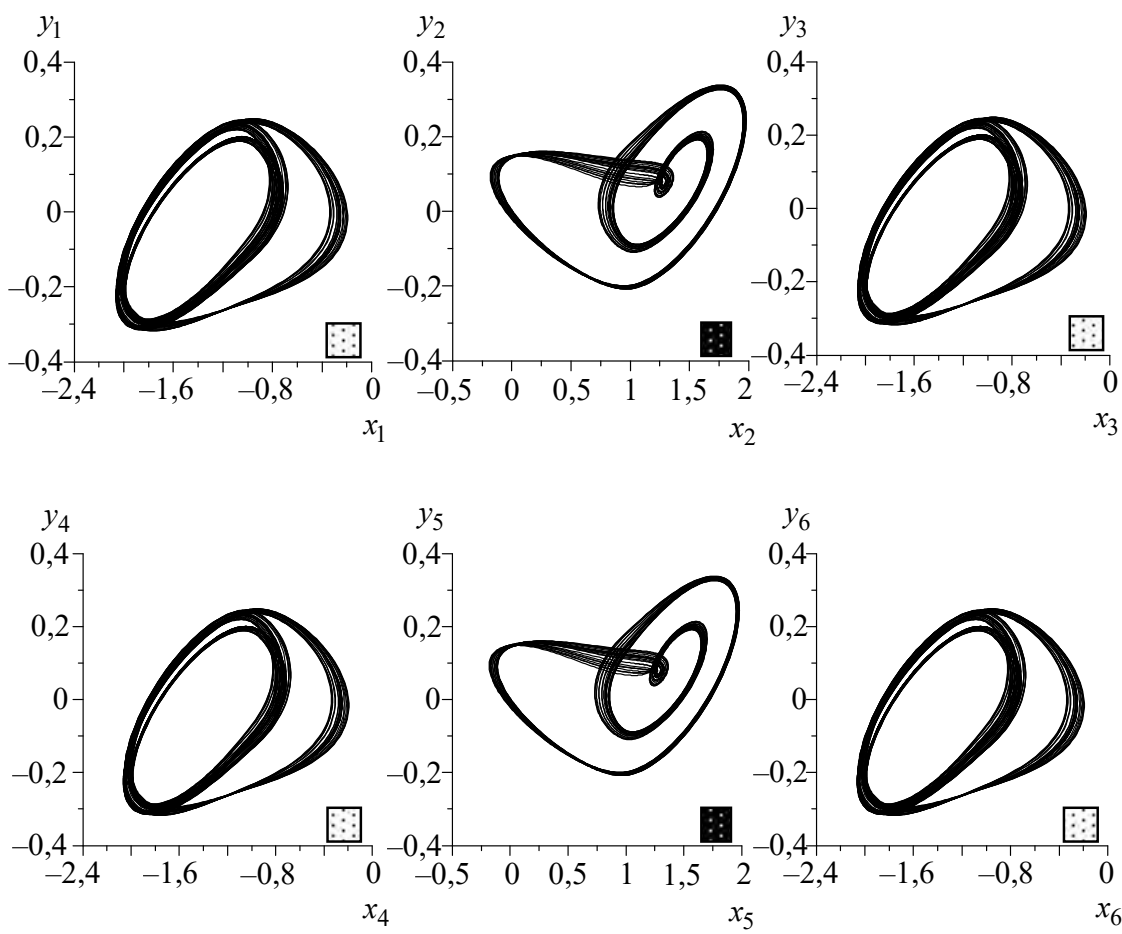

Рис. 6.10. Кластерная структура в цепочке из $N=6$ элементарных осцилляторов Чуа как синхронизация двух ПК $O_{a}(2)$.

Как видим, КС на рис. 6.10 «разрезается» на пару блоков - пару синхронизованных ПК.

Подведем итог. Во-первых, явление «cluster synchronization»случай классической синхронизации в общепринятом понимании этого процесса и в этом смысле не является новым физическим явлением. Во-вторых, на примере систем с небольшим числом осцилляторов определились два типа субъектов, классическая синхронизация которых порождает кластерные структуры в цепочке динамических систем. Рассмотренные частные случаи субъектов кластерных структур с малым числом элементов естественным образом переносятся на общий случай, а именно: первый субъект кластерных структур в цепочках - КО типа $O_{s}(n)$; второй - прос- 
тая клетка (ПК) как структурированный объект, возникающий на базе синхронизации пары КО типа $O_{a}(n)$. Как будет показано в следующих разделах, эти два типа субъектов исчерпывают все возможные типы кластерных структур в цепочках осцилляторов, являющихся простейшим видом решеток.

Замечание. Собственная структура и роль $O_{s}(n)$ и $O_{a}(n)$ в формировании кластерных структур невольно навязывает аналогию с квантово-механическими квазичастицами - бозонами и фермионами. Как известно, первые играют во взаимодействиях самостоятельную роль, а вторые, прежде чем стать субъектами взаимодействий, предварительно объединяются в пары (рождаются и умирают парами). В рамках данных представлений можно предложить квантово-механическую интерпретацию кластерной динамики решетки.

По свойствам числа $N$ (делимость), а также свойствам $O_{s}(n)$ и $O_{a}(n)$ можно установить все интегральные многообразия в фазовом пространстве решетки и в качестве энергетических уровней упорядочить их по числу кластеров. Как будет показано ниже, большая часть (или все) таких уровней метастабильна. Это значит, что (типичный случай) при передаче порции энергии осцилляторам решетки (начальные условия), соответствующей верхнему энергетическому уровню, взаимодействия частиц (элементарных осцилляторов) приведет к рождению ансамбля взаимодействующих квазичастиц - кластерной структуры определенного типа, соответствующей «верхнему» многообразию. Поскольку уровень неустойчив, то через некоторое время данное состояние решетки исчезает. В силу диссипативности системы часть энергии поглощается - система переходит на более низкий энергетический уровень, к другой метастабильной кластерной структуре, и т. д., вплоть до уровня, соответствующего интегральному многообразию $M_{0}$. В целом картина может напоминать процесс трансформации возмущенного гидродинамического течения в ламинарное состояние через последовательность сменяющих друг друга структур. 


\section{2. Синтез и общие свойства схем кластерных структур}

Как известно, эквивалентные преобразования в электрических цепях - широко применяемый метод анализа и расчета электрических схем, особенно эффективный в тех случаях, когда эти схемы громоздки и, значит, мало доступны для данных процедур в первоначальном виде. Напомним, что эквивалентными преобразованиями электрической цепи называют такие преобразования некоторой части ее электрической схемы, при которых все токи участков цепей, а также потенциалы всех точек за пределами преобразуемой части остаются неизменными в процессе преобразований. Сказанное имеет непосредственное отношение к схемам кластерных структур (на простые случаи обращалось внимание в предыдущем параграфе) по следующим причинам. Во-первых, решетки, элементами которых являются радиотехнические автогенераторы (сверхпроводящие переходы в их числе), также являются предметом нашего интереса. Во-вторых, при анализе кластерных структур в решетках другой физической природы всегда можно сопоставить им некоторый радиотехнический аналог. В указанном смысле любую схему кластерной структуры можно рассматривать в качестве блок-схемы гипотетической решетки радиотехнических автогенераторов, где цветом отражается индивидуальный динамический режим этих элементов схемы.

Надо сказать, что выясненная нами природа кластерных структур - синхронизация обобщенных осцилляторов и простых клеток фактически предопределяет правила синтеза схем кластерных структур, свойства этих схем и их эквивалентные преобразования, действующие вне зависимости от геометрической размерности решеток и их формы. Дадим список словесных определений, общих принципов внутреннего устройства, а также элементарных (эквивалентных) преобразований схем кластерных структур.

1. Два непосредственно связанных, синхронизованных элементарных осциллятора, изображенные на схеме кластерной структуры одним цветом, будем называть разрезаемой парой (РП). Связь между такими осцилляторами может быть удалена - разрезана (на электрической схеме это соответствует разрезанию обесточенных участков цепи - эквипотенциальных точек). Наоборот, между одноцветными изображениями схемы может быть установлена любая пассивная связь и, в частности, произведено их короткое замыкание.

2. Если схема имеет хотя бы одну РП, то ее будем называть разрезаемой, в противном случае - неразрезаемой. 
3. Схема кластерной структуры простой клетки является неразрезаемой.

4. Если некоторая схема не имеет ни одной РП, то это или схема простой клетки, или схема отдельного КО, или эта схема неправильна.

5. Процедуру совмещения двух и более одноцветных изображений путем перегиба схемы через оси симметрии или другим способом будем называть свертылванием схемь (на электрической схеме это соответствует процедуре замыкания эквипотенциальных точек).

6. Схема простой клетки свертывается к схеме ее базового КО, т. е. к схеме, не содержащей синхронизованных (одноцветных) элементарных осцилляторов.

7. Любая схема кластерной структуры, если она не является схемой одной простой клетки или схемой отдельного КО, при разрезании всех РП распадается на идентичные схемные блоки с изображением схемы базового КО (бозона) или схемы базовой простой клетки (фермиона).

На базе этих простых принципов и столь же простых элементарных преобразований схем (школьная физика) далее будут выяснены все возможные типы кластерных структур в цепочке и кольце элементарных осцилляторов, а также будут доказаны математически нетривиальные утверждения, касающиеся полноты совокупностей этих типов структур.

Обратимся к вопросам синтеза схем кластерных структур. Подобно соединению проводников, соединение схем КО как частей полной схемы кластерной структуры, изображенное на рис. 6.11, называем последовательным (последовательный синтез схемы кластерной структуры).

Матрицу $\mathbf{D}=\operatorname{diag}\left(d_{11}, d_{22}, \ldots d_{s s}\right), \quad$ в которой диагональные элементы $d_{i i}$ равны «0» или «1», будем называть матрицей синтеза схемы кластерной структуры. Индексы единичных элементов определяют номера связанных элементарных осцилляторов как элементов КО или ПК. Размерность этой матрицы равна числу осцилляторов, образующих КО $(m=n$, рис. $6.11, \mathrm{a})$ или ПК ( $m=2 n-1$, рис. $6.11, б)$. Матрицей последовательного синтеза является $\mathbf{D}=\mathbf{C}^{*}=\operatorname{diag}(1,0, \ldots 0)$ или $\mathbf{C}_{*}=\operatorname{diag}(0,0, \ldots 1)$ в зависимости 
от порядка следования нуль-векторов элементарных осцилляторов, являющихся координатами векторов КО или ПК.
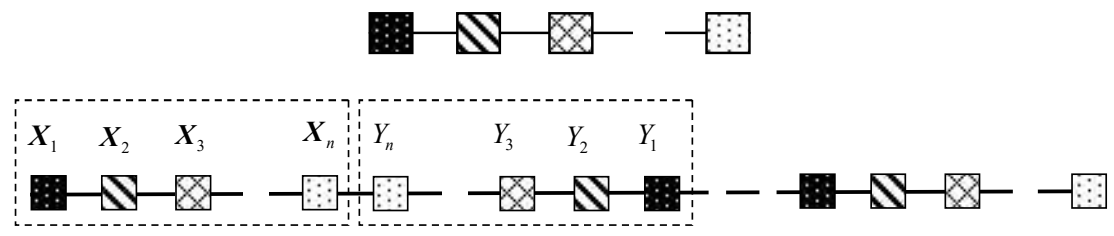

a
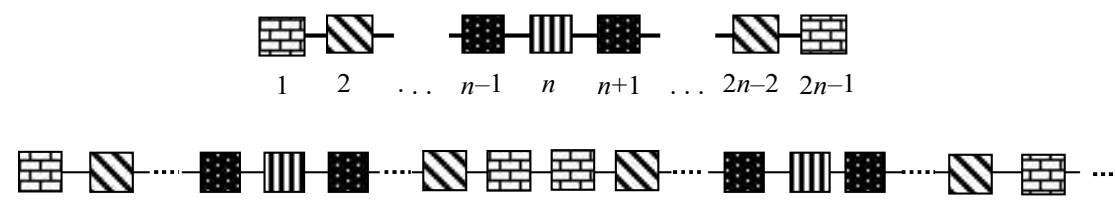

$1 \quad 2 \ldots n-1 \quad n \quad n+1 \ldots 2 n-2 \quad 2 n-1 \quad 2 n \quad 2 n+1 \ldots$

б

Рис. 6.11. Схема КО $O_{s}(n)$ и последовательный синтез схемы кластерной структуры на его базе (а); изображение простой клетки КО $O_{a}(n)$

и последовательный синтез схемы кластерной структуры на ее базе (б).

Соединение, изображенное на рис. 6.12, будем называть параллельным.

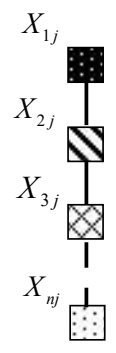

a

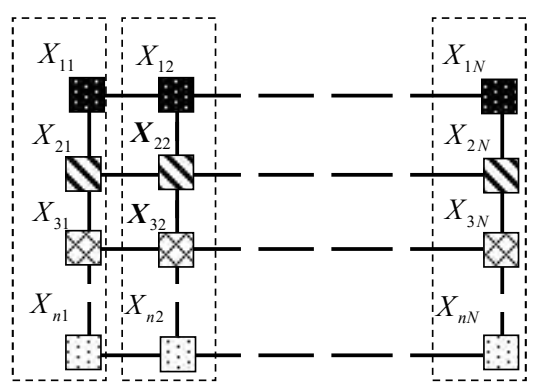

6

Рис. 6.12. Схема КО $O_{S}(n)$ (a); параллельный синтез - ленточная кластерная структура в двумерной решетке (б). 
В данном случае матрицей синтеза является $\mathbf{D}=\operatorname{diag}(1,1, \ldots, 1)=\mathbf{I}-$ единичная матрица.

Все другие типы синтеза блочных схем из идентичных схемблоков (КО или ПК) относим к смешанному типу (рис. 6.13).
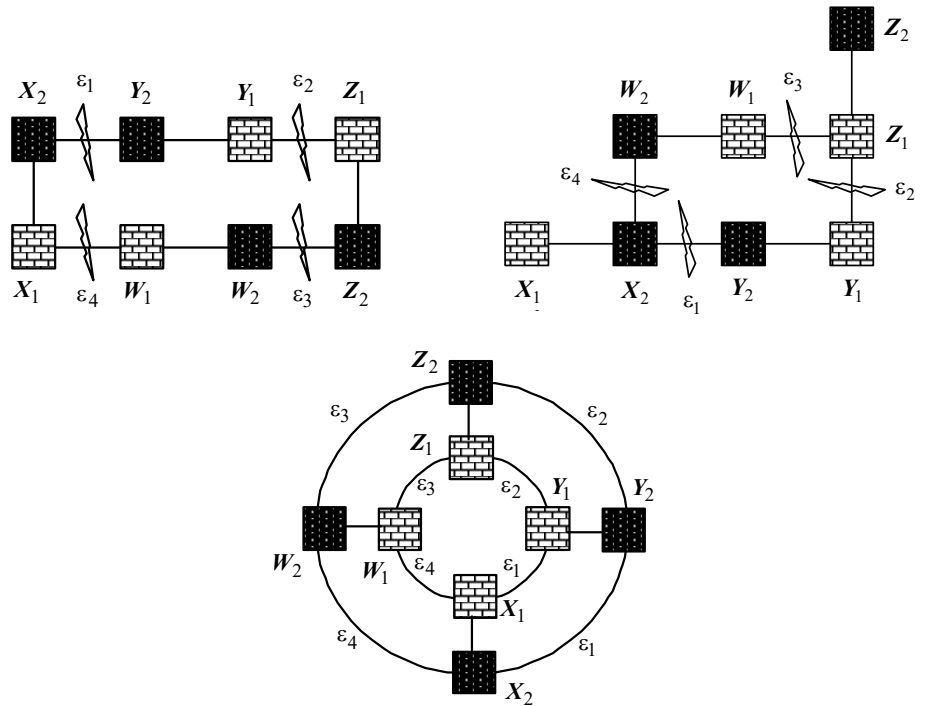

Рис. 6.13. Примеры смешанного синтеза КС на базе КО $O_{s}(2)$.

Еще раз обратим внимание на особенности существования и сосуществования кластерных структур в однородных решетках: если синтезированная схема кластерной структуры однозначно определяет и саму решетку (железо), то в общем случае это не значит, что в полученной материальной решетке заданная структура будет единственной. Синтезированная структура, как и сопутствующие ей структуры, связаны с определенными начальными условиями полученной решетки. Так, если начальные условия решетки будут заданы в соответствии со схемой (одинаковые или достаточно близкие к одинаковым начальные условия для одноцветных осцилляторов), то и далее ее динамика будет протекать в соответствии с синтезированной схемой. При других начальных условиях результат может быть иным. Можно сказать, что структуры в однородных решетках обусловлены упорядоченной неоднородностью начальных условий. 
В связи с вышесказанным возникают вопросы о синтезе структур в решетках путем внедрения заранее заданной упорядоченной неоднородности в материальную часть решетки. Все вопросы синтеза упорядоченно неоднородных решеток с соответствующей кластерной динамикой имеют положительное решение, причем в рамках тех же самых принципов и приемов, что и в однородных решетках. Для этого достаточно считать, что рабочий режим (аттрактор) КО обусловлен не начальными условиями, а неоднородностью параметров образующих его элементарных осцилляторов. Синтезированная кластерная структура в подобной решетке может обладать глобальной устойчивостью и управляемостью, включая дистанционное управление.

Приведем еще два примера «кластеризации» достаточно необычных объектов - объектов с фрактальными свойствами. Они являются одним из передовых направлений современных теоретических и прикладных исследований $[87,88]$.

Дерево Кейли. Как математический объект, дерево Кейли формируется следующим образом. Строится фигура в виде равносторонней буквы «Н», затем к каждому из концов фигуры надстраивается подобная фигура с коэффициентом подобия $1 / 2$. Процесс построения бесконечен. Три итерации показаны на рис. 6.14,a. На практике дерево получают путем напыления или травления тонкой пленки [89]. Естественно, на практике число итераций конечно и определяется уровнем технологического процесса, т. е. физические объекты имеют псевдофрактальную структуру, однако их свойства близки к свойствам настоящих фракталов.

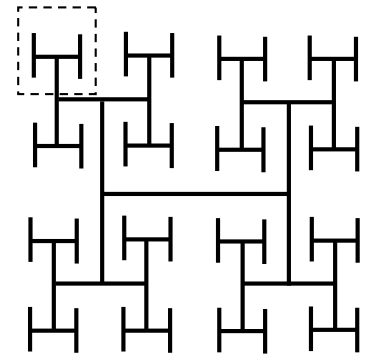

a

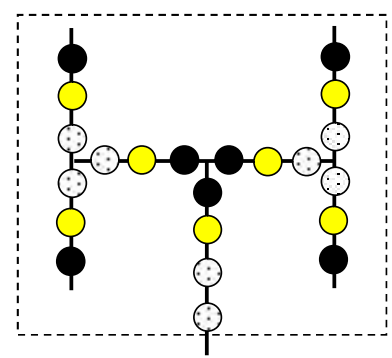

6

Рис. 6.14. Дерево Кейли (а) и фрагмент кластеризованного дерева (б). 
Допустим, что технология позволяет конструировать некоторые активные нелинейные элементы (условно - осцилляторы) достаточно малых размеров. В таком случае, взяв за основу схему некоторого КО цепочки с числом элементов $n$ или схему простой клетки с числом элементов $2 n-1$, «выкладываем» эту схему (по правилам синтеза) на каждой ветке минимального элемента дерева (на рис. 6.14,a обозначен пунктиром) нужное число раз. Тем самым определяем нужное число условных осцилляторов для размещения на каждой ветке элемента дерева. Таким же образом синтез структуры распространяется на все дерево. В результате получаем «кластеризованное» дерево, фрагмент которого изображен на рис. 6.14,б, где показана структура на базе $O_{s}(3)$. Однако могут быть выбраны и другие КО или ПК.

Теперь допустим, что все названные условные осцилляторы идентичны. В таком случае мы будем иметь дело с однородной решеткой, хотя и отличающейся от предыдущих примеров своей сверхоригинальной формой. Физика остается прежней. Знаем, что наряду с построенной кластерной структурой в данной решетке потенциально возможны и другие структуры. По этой причине для реализации нужного процесса необходимо иметь техническую возможность (умение) задавать вполне определенные начальные условия решетки (перед ее запуском в работу). Кроме того, в случае неустойчивости данной структуры потребуются дополнительные меры для поддержания ее метастабильного состояния. Поскольку контроль начальных условий - практически нерешаемая задача, то, скорее всего, все данное предприятие лишено практического смысла. Совсем иная ситуация может сложиться, если осцилляторы будут управляемы по какому-либо параметру, например по частоте или фазе колебаний. В этом случае мы будем иметь дело с упорядоченно неоднородной решеткой, в которой кластерный динамический режим, с одной стороны, будет устойчив, а с другой - будет иметь управляемые параметры. К тому же, управление параметрами осцилляторов создаст возможность переключения решетки с одной кластерной структуры на другую. Возможно, что в случае антенн с фрактальной решеткой подобным образом можно будет получать диаграммы направленности с новыми необычными свойствами.

Ковер Серпинского. Этот объект строится следующим образом: квадрат делится на девять равновеликих квадратов, серединный 
квадрат удаляется, а с оставшимися квадратами процедура деления повторяется. Процесс бесконечен. Получающаяся в результате фигура - ковер Серпинского. Три итерации квадрата изображены на рис. 6.15,a. Незаконченный ковер обозначен черным цветом, а белым - удаленные части квадрата.

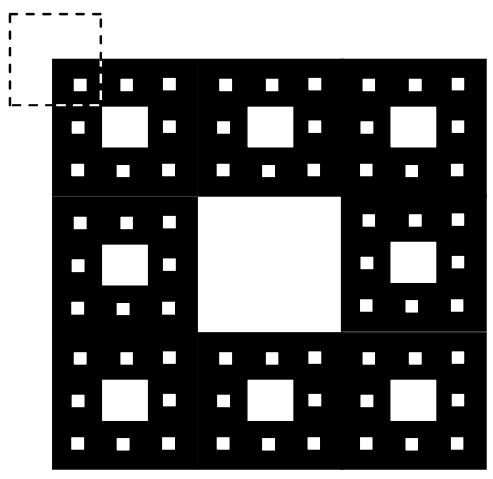

a

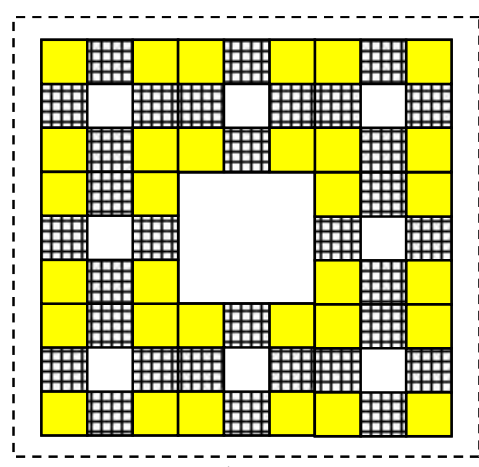

б

Рис. 6.15. Ковер Серпинского (а) и фрагмент кластеризованного ковра (б).

Как и в предыдущем примере, выбираем наименьший элемент ковра - квадрат, выделенный на рис. 6.15,a пунктиром, а также схему нужного КО или ПК. Их размеры, определяемые возможностями технологии изготовления как условных осцилляторов, так и «тела» решетки, должны соответствовать друг другу. На рис. 6.15,б в качестве базового элемента выбрана схема квадратной ПК $(3 \times 3)$ с двумя кластерами. Следуя правилу, синтезируем схему кластерной структуры в данном элементе, а затем и на всей поверхности ковра. Фрагмент кластерной структуры показан на рис. 6.15,б. Связи элементарных осцилляторов изображаются соприкосновением сторон квадратов. О свойствах получаемых структур в однородных и неоднородных решетках сказано выше.

При синтезе неоднородных решеток с заданной кластерной структурой элементарные осцилляторы, составляющие КО и, соответственно, ПК, могут различаться не только по параметрам, но быть и разных типов. Это не меняет изложенные выше правила синтеза схем кластерных структур.

Приведем пример для иллюстрации сказанного. На рис. 6.16 представлены индивидуальные фазовые портреты трех осциллято- 
ров - «кандидатов» для КО $O_{s}(3)$. Первый и второй - осцилляторы Чуа с параметрами $\left(\beta, \gamma, m_{0}, m_{1}\right)=(14 ; 0,1 ;-1 / 7 ; 2 / 7)$ и $\alpha_{1}=9$, $\alpha_{2}=9,5$ соответственно. Третий - осциллятор Лоренца с параметрами $\sigma=10, r=24$ и $b=1$.
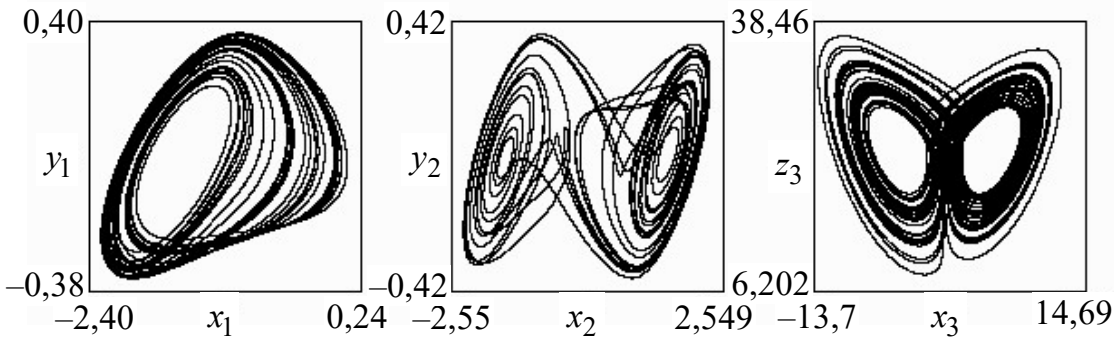

Рис. 6.16. Хаотические аттракторы осцилляторов до образования КО $O_{s}(3)$.

На рис. 6.17 изображены проекции кластерного аттрактора КО $O_{s}(3)$ на координатные плоскости. Матрица связи элементарных осцилляторов $\varepsilon \mathbf{C}=0,5 \mathrm{I}$. На рис. 6.18 показан режим синхронизации пары КО с матрицей и скалярным параметром связи $\varepsilon \mathbf{C}_{*}=25 \operatorname{diag}(0,0,1)$.
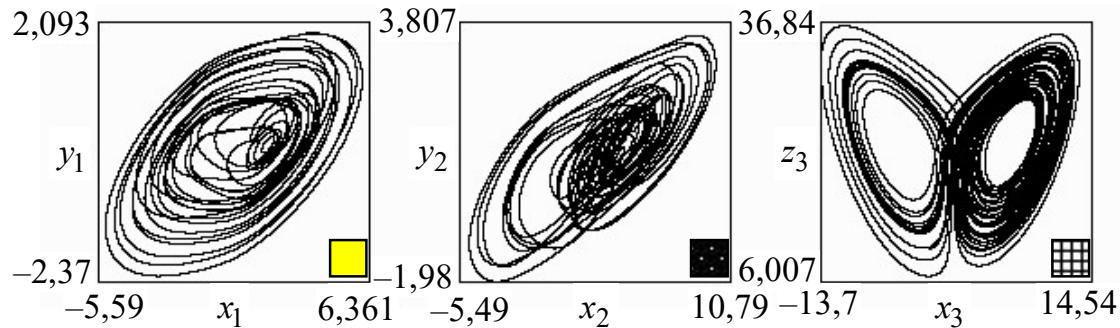

Рис. 6.17. Проекции кластерного аттрактора на координатные плоскости.

Визуального сравнения фазовых портретов осцилляторов достаточно для утверждения того, что мы имеем дело с синхронизацией пары КО. Синхронизация устойчива.

В заключение этого раздела приведем пример из биологии [90]. 

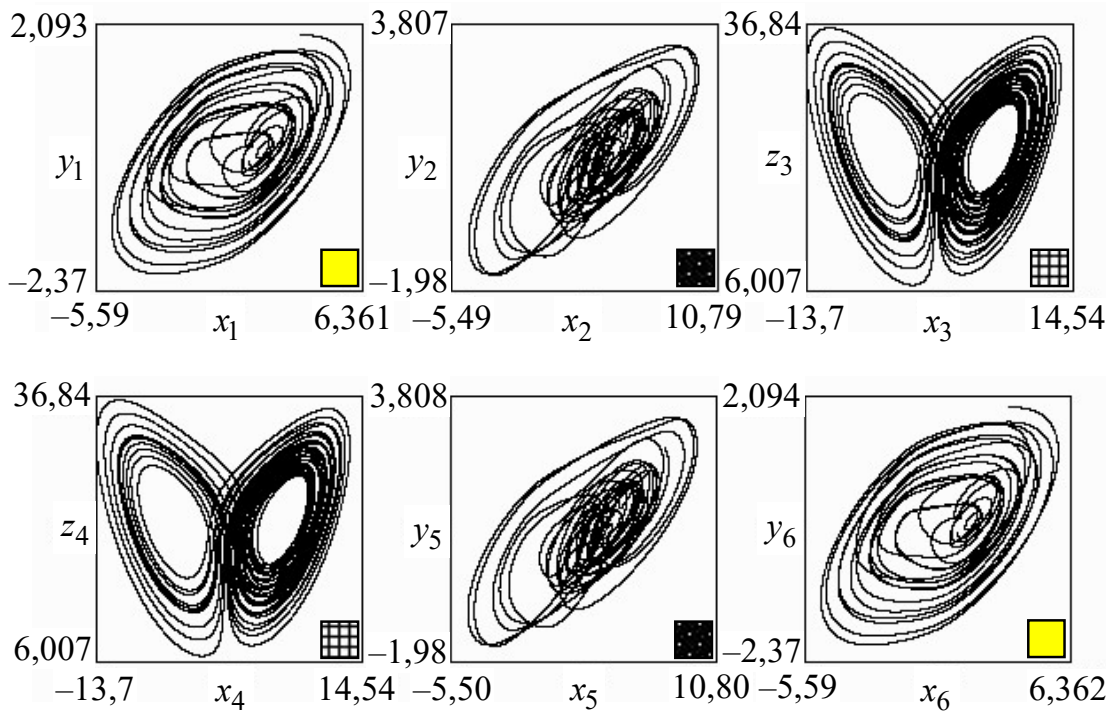

Рис. 6.18. Кластерная структура как синхронизация пары КО $O_{s}(3)$.

В развернутом виде молекула ДНК имеет вид лестницы, состоящей из отдельных звеньев (в действительности она скручена в спираль). Число звеньев $\sim 10^{5}$. Фрагмент этой лестницы изображен на рис. 6.19,a. На рисунке: (C) - молекулы сахара, Ф)- молекулы фосфорной кислоты. $\langle\mathrm{T},\langle\mathrm{A}\rangle,\langle\mathrm{G},\langle\mathrm{C}\rangle$ - нуклеотиды тимин, аденин, гуанин и цитозин соответственно [91]. В данном случае нуклеотидам отведем роль условных элементарных осцилляторов. Сахара и фосфаты отнесем к элементам «связи» условных осцилляторов $\mathrm{T}$, $\mathrm{A}, \mathrm{G}$ и C. При таких условиях схема кластерной структуры фрагмента лестницы изображена рис. 6.19,б. Из рисунка видно, что схема структуры не имеет ни одной РП, т. е. является неразрезаемой, следовательно, может представлять схему структуры простой клетки. В таком случае путем коротких замыканий условных эквипотенциальных точек она должна свертываться к одному КО. В данном случае процедура свертывания ПК геометрически соответствует наложению двух блоков после поворота второго на $180^{\circ}$. Таким образом, блок (T, A, G, C) представляет КО, а структура на рис. 6.19,а является кластерной структурой простой клетки. 

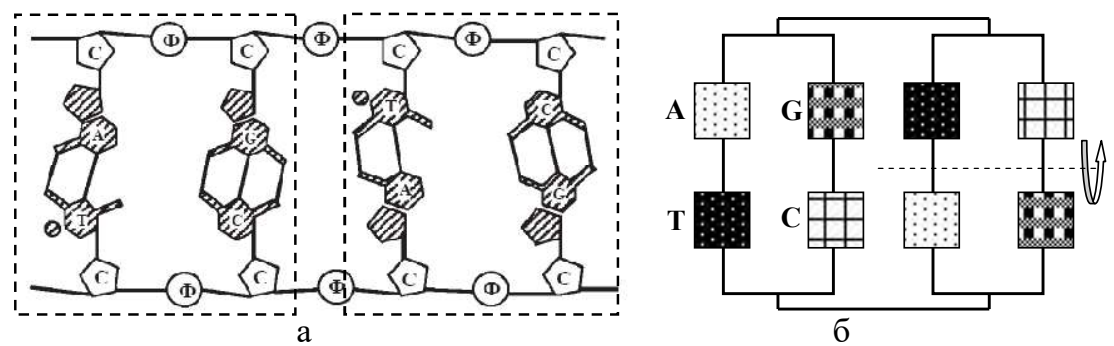

Рис. 6.19. Фрагмент модели развернутой молекулы ДНК.

\section{3. К-осцилляторы цепочки и полнота типов ее кластерных структур}

С точки зрения кластерных структур однородная цепочка представляет собой наиболее простой объект, ее исследованиям в приложениях к различным физическим системам и явлениям посвящено немалое число публикаций [64, 73, 75, $92-94$ и др.]. Ниже, находясь в рамках интерпретации кластерных структур как взаимной синхронизации обобщенных осцилляторов, мы покажем (докажем), что все кластерные структуры в цепочке ограничиваются всего лишь двумя типами. Причем это будет относиться как к однородным, так и упорядоченно неоднородным цепочкам.

Дадим общее математическое определение упоминавшегося ранее КО типа $O_{s}(n)$.

Определение 6.1. К-осциллятором типа $O_{s}(n)$ называем динамическую систему $n$ связанных элементарных осцилляторов вида

$$
\begin{aligned}
& \dot{\mathbf{V}}=\mathbf{H}(\mathbf{V}), \\
& \mathbf{V}=\left(\mathbf{X}_{1}, \mathbf{X}_{2}, \ldots, \mathbf{X}_{n}\right)^{T}, \\
& \mathbf{H}(\mathbf{V})=\left(\mathbf{F}_{1}\left(\mathbf{X}_{1}\right), \mathbf{F}_{2}\left(\mathbf{X}_{2}\right), \ldots, \mathbf{F}_{n}\left(\mathbf{X}_{n}\right)\right)^{T}-\varepsilon\left(\mathbf{B}_{s} \otimes \mathbf{C}\right) \mathbf{V},
\end{aligned}
$$




$$
\mathbf{B}_{s}=\left(\begin{array}{ccccccc}
1 & -1 & 0 & \cdots & \cdots & 0 & 0 \\
-1 & 2 & -1 & \ddots & \cdots & 0 & 0 \\
0 & -1 & 2 & \ddots & \ddots & 0 & 0 \\
\vdots & \ddots & \ddots & \ddots & \ddots & \ddots & \vdots \\
\vdots & \vdots & \ddots & \ddots & 2 & -1 & 0 \\
0 & 0 & 0 & \ddots & -1 & 2 & -1 \\
0 & 0 & 0 & \cdots & 0 & -1 & 1
\end{array}\right)
$$

при условии существования в фазовом пространстве $G(\mathbf{V})$ аттрактора $A_{s}(n)$ такого, что для $\forall\left(\mathbf{X}_{1}, \mathbf{X}_{2}, \ldots, \mathbf{X}_{n}\right) \in A_{s}(n), \quad \mathbf{X}_{i} \neq \mathbf{X}_{j}$, $i \neq j, i, j=1,2, \ldots, n . \mathbf{B} \otimes \mathbf{C}$ - матрица связи элементарных осцилляторов.

Элементарные осцилляторы в уравнении (6.11) могут быть как идентичными, что соответствует однородной цепочке, так и неидентичными по своим параметрам или даже по своей природе. Связь осцилляторов в (6.11) принята однородной и локальной, хотя это обстоятельство также не принципиально и не меняет общей сути дела.

Записывая (6.11) покоординатно, нетрудно убедиться, что выражение соответствует отрезку цепочки с граничными условиями Неймана, которые в (6.11) выполняются «автоматически», поскольку заложены в матрицу связи. Для удобства на рис. 6.20 повторно приведена схема КО $O_{s}(n)$ в «рабочем» динамическом режиме (на аттракторе $A_{s}(n)$ ), а на рис. 6.21 воспроизведен синтез схемы кластерной структуры в цепочке на базе этого КО.

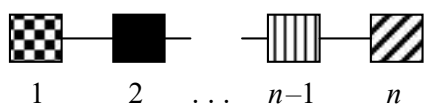

Рис. 6.20. Схема $O_{s}(n)$. 


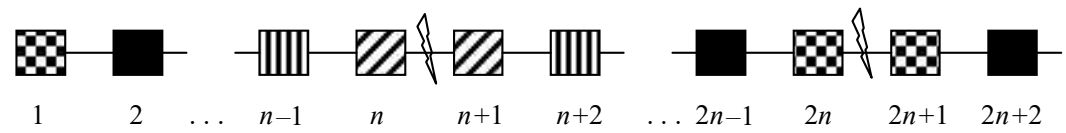

Рис. 6.21. Синтез схемы кластерной структуры на базе $O_{s}(n)$.

По полной аналогии дадим определение КО $O_{a}(n)$.

Определение 6.2. К-осциллятором типа $O_{a}(n)$ называем систему $n$ связанных элементарных осцилляторов вида

$$
\begin{aligned}
& \dot{\mathbf{V}}=\mathbf{H}(\mathbf{V}), \\
& \mathbf{V}=\left(\mathbf{X}_{1}, \mathbf{X}_{2}, \ldots, \mathbf{X}_{n}\right)^{T} \text {, } \\
& \mathbf{H}(\mathbf{V})=\left(\mathbf{F}_{1}\left(\mathbf{X}_{1}\right), \mathbf{F}_{2}\left(\mathbf{X}_{2}\right), \ldots, \mathbf{F}_{n}\left(\mathbf{X}_{n}\right)\right)^{T}-\varepsilon\left(\mathbf{B}_{a} \otimes \mathbf{C}\right) \mathbf{V}, \\
& \mathbf{B}_{a}=\left(\begin{array}{ccccccc}
1 & -1 & 0 & \cdots & \cdots & 0 & 0 \\
-1 & 2 & -1 & \ddots & \cdots & 0 & 0 \\
0 & -1 & 2 & \ddots & \ddots & 0 & 0 \\
\vdots & \ddots & \ddots & \ddots & \ddots & \ddots & \vdots \\
\vdots & \vdots & \ddots & \ddots & 2 & -1 & 0 \\
0 & 0 & 0 & \ddots & -1 & 2 & -1 \\
0 & 0 & 0 & \cdots & 0 & -2 & 1
\end{array}\right)
\end{aligned}
$$

при условии существования в фазовом пространстве $G(\mathbf{V})$ аттрактора $A_{a}(n)$ такого, что для $\forall\left(\mathbf{X}_{1}, \mathbf{X}_{2}, \ldots, \mathbf{X}_{n}\right) \in A_{a}(n), \quad \mathbf{X}_{i} \neq \mathbf{X}_{j}$, $i \neq j, \quad i, j=1,2, \ldots, n . \mathbf{B}_{a} \otimes \mathbf{C}$ - матрица связи элементарных осцилляторов.

Относительно смысла переменных параметров и нелинейных функций уравнения (6.12) сказано выше.

Напомним, что КО данного типа существуют только в паре. Взаимная синхронизация такой пары в предельном (жестком) случае связи двух КО между собой определяет кластерную структуру ПК. В свою очередь, образовавшиеся простые клетки являются потенциальными субъектами кластерных структур в цепочке. Заметим, что в результате жесткой связи пары концевых элементарных 
осцилляторов КО происходит фактическое их объединение и, как следствие, вырождение числа степеней свободы связанной системы на число степеней свободы одного элементарного осциллятора. При этом число действующих элементарных осцилляторов в простой клетке становится равным $2 n-1$. Уравнения простой клетки являются уравнениями отрезка цепочки с неймановскими граничными условиями и с числом элементарных осцилляторов $2 n-1$. Синтез схемы кластерной структуры ПК можно интерпретировать в виде присоединения к схеме $O_{a}(n)$ ее зеркального образа (рис. 6.22). Жирная черта на рисунке - символическое зеркало. На рис. 6.23 воспроизведен синтез схемы КС в цепочке на базе схемы ПК $O_{a}(n)$.

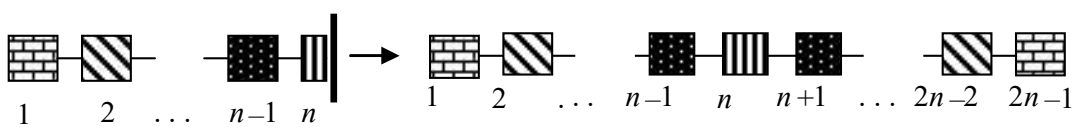

Рис. 6.22. Схема КО $O_{a}(n)$ и синтез схемы КС простой клетки на ее базе.

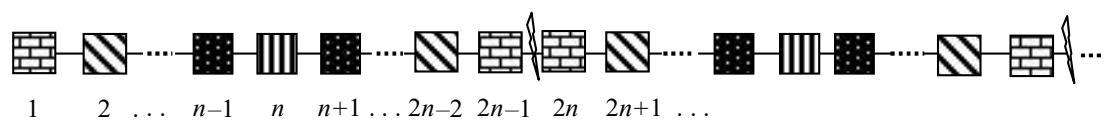

Рис. 6.23. Синтез схемы КС в цепочке на базе схемы простой клетки $O_{a}(n)$.

Как уже говорилось, в отношении общих принципов теории кластерных структур деление систем на осцилляторы и ротаторы не имеет смысла. Значимость такого деления появляется только при исследовании конкретных систем, их аттракторов, когда системы ротаторов проявляют специфические особенности, связанные с цилиндричностью фазового пространства. По этой причине и далее сохраняем принятую терминологию и обозначения; говоря об осцилляторах, будем иметь в виду, что то же самое относится и к ротаторам. Добавим к сказанному иллюстрации численного эксперимента по кластерным структурам в цепочке ротаторов, физический пример которой изображен на рис. 6.24 . 


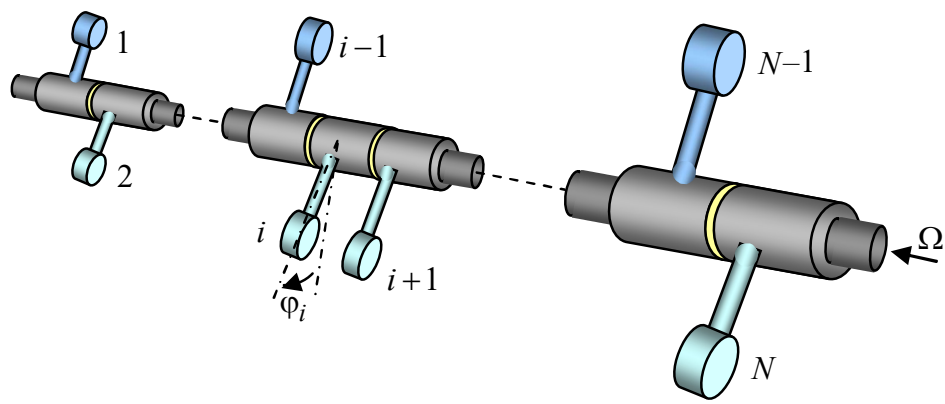

Рис. 6.24. Цепочка маятников Фруда со структурированной динамикой.

Численно исследовалась цепочка ротаторов с векторами динамического состояния элементов: $\mathbf{X}_{i}=\left(\varphi_{i}, x_{i}\right)^{T}$ и $\mathbf{F}\left(\mathbf{X}_{i}\right)=$ $=\left(x_{i},-\lambda x_{i}-\sin \varphi_{i}+\gamma+A \sin \omega_{0} \tau\right)^{T}$ со скалярным параметром связи $\sigma$ и матрицей $\mathbf{C}=\left(\begin{array}{ll}0 & 0 \\ 0 & 1\end{array}\right)$. В частности, она представляет динамику изображенной на рис. 6.24 цепочки маятников Фруда.

1. Кластерные структуры автономной иеепоки. Организовать демонстрационные примеры кластерных структур в цепочке ротаторов не составляет труда. Например, в области параметров (2) (см. рис. 1.1) одиночный ротатор в зависимости от начальных условий может пребывать или в состоянии равновесия, или во вращательном движении. Это значит, что в фазовом пространстве пары связанных ротаторов существует устойчивый вращательно-колебательный предельный цикл (по крайней мере, при малой связи это гарантировано непрерывностью решений по параметру). Он и является одним их кластерных аттракторов системы (рис. 6.25). Первый из связанных маятников вращается, второй испытывает незначительные колебания в окрестности нижнего состояния равновесия.

Далее исследовалась цепочка из 8 элементов, в которой при тех же параметрах получена кластерная структура (рис. 6.26). Забегая вперед, скажем, что она является устойчивой. 

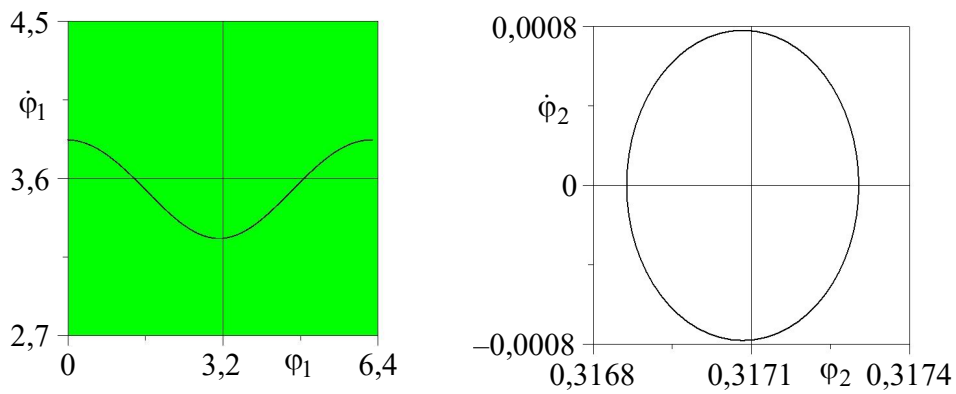

Рис. 6.25. Проекции аттрактора $A_{S}(2)$ на координатные плоскости КО $O_{S}(2): \lambda=0,07, \gamma=0,28, \sigma=0,09$.
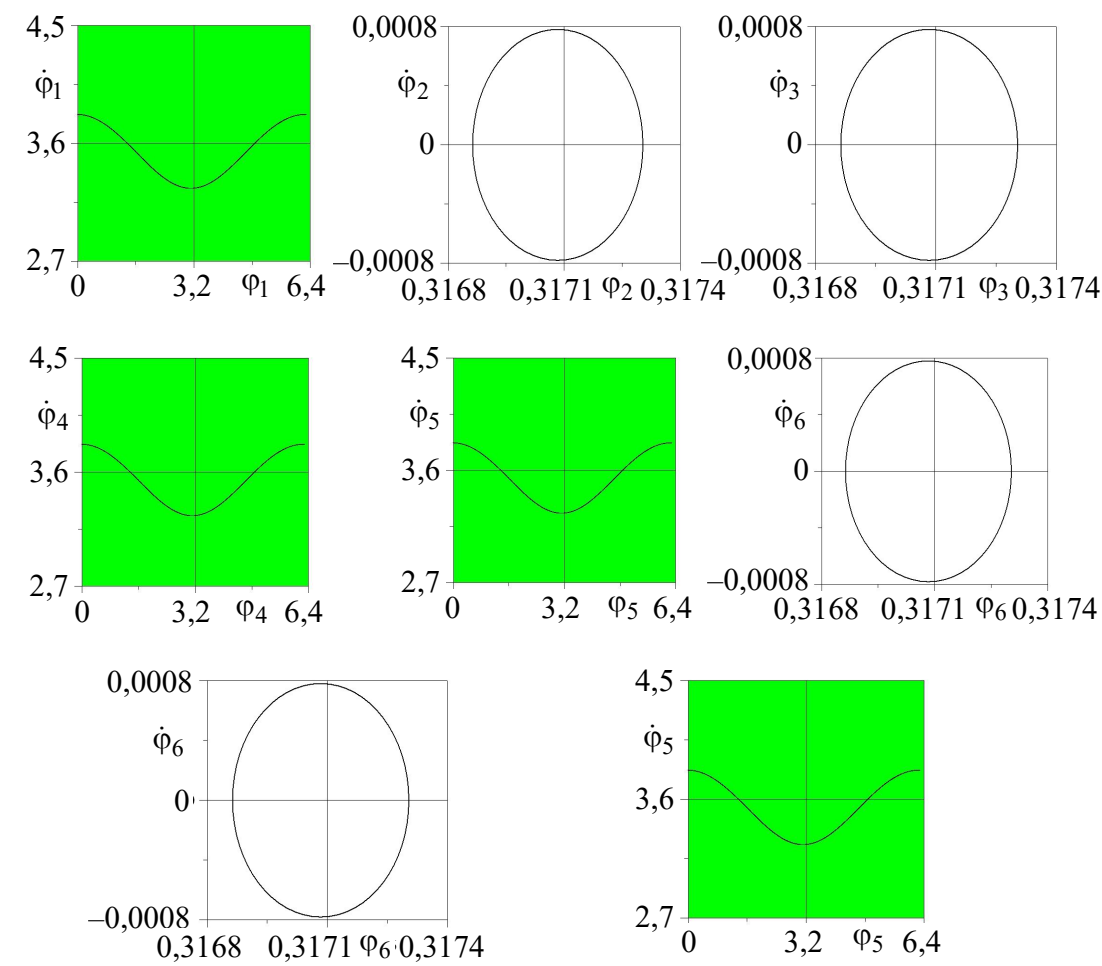

Рис. 6.26. Кластерная структура на базе синхронизации четырех КО $O_{s}(2)$ в цепочке из $N=8$ элементарных ротаторов. 
Для параметров индивидуальных ротаторов из области (2) (см. рис. 1.1) по определению 6.2 смоделирован КО $O_{a}(2)$. Его аттрактор $A_{a}(2)$ с параметрами связанной системы $\lambda=0,07$, $\gamma=0,28, \sigma=0,29$ представлен на рис. 6.27. Схожесть с аттрактором $A_{s}(2)$ только внешняя, они принципиально разные.
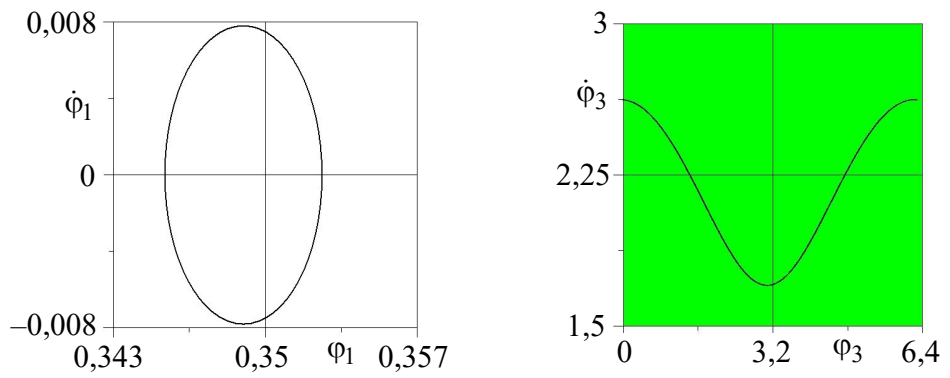

Рис. 6.27. Проекции аттрактора $A_{a}(2)$ на одноименные координатные плоскости КО $O_{a}(2)$.

На рис. 6.28 изображена кластерная структура простой клетки КО $O_{a}(2)$ (устойчивая), полученная при тех же параметрах в цепочке из трех элементов.
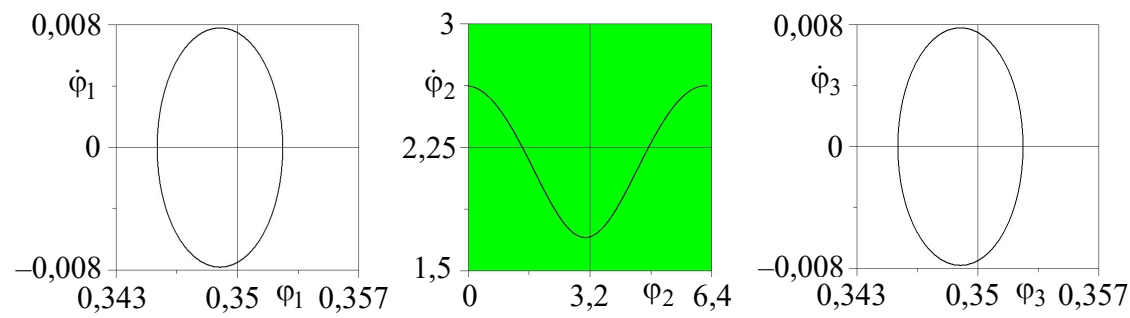

Рис. 6.28. Кластерная структура простой клетки КО $O_{a}(2)$.

Далее при тех же параметрах исследовалась цепочка из 6 элементов, в которой получена кластерная структура, изображенная на рис. 6.29. 

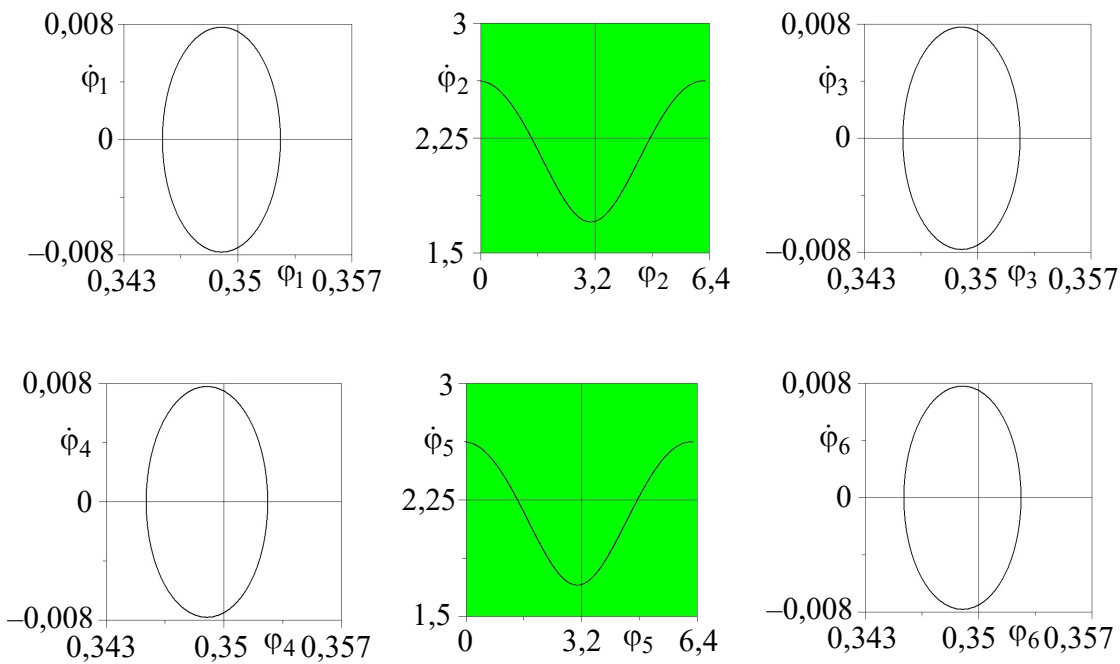

Рис. 6.29. Устойчивая кластерная структура как синхронизация двух простых клеток $O_{a}(2)$.

2. Кластерные структуры неавтономной цепочки. В данном случае для выбора параметров, необходимых для существования кластерных аттракторов, обращаемся к бифуркационной диаграмме, изображенной на рис. 1.7,а. Как видно из рисунка, подходящими являются параметры из всех областей, кроме области (1): для этих параметров наблюдается глобально устойчивая синфазная синхронизация, а нам необходима различная парциальная динамика ротаторов.

Пример кластерного аттрактора $A_{S}(2)$ показан на рис. 6.30 . Параметры счета: $\lambda=0,07, \gamma=0,51, \sigma=0,079, A=0,28, \omega=0,56$. В данном случае кластерным аттрактором является трехмерный тор $T^{3}$, соответствующий режиму биений ротаторов как между собой, так и с периодической внешней силой, т. е. отсутствию как взаимной, так и принудительной синхронизации. Далее без комментариев приведем результаты численного эксперимента (рис. 6.316.34). Все кластерные структуры являются устойчивыми. 

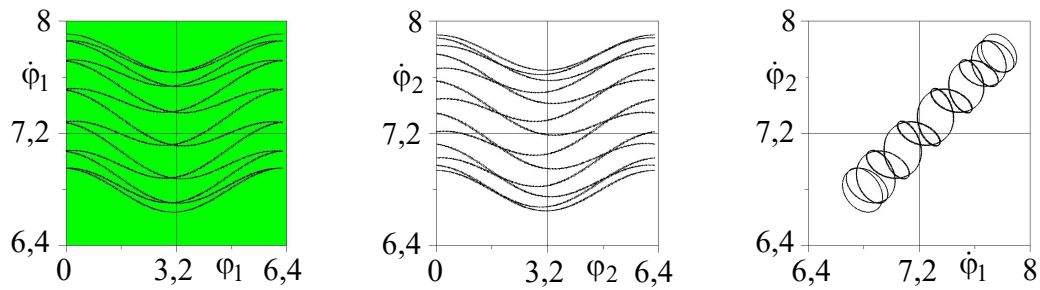

Рис. 6.30. Проекции аттрактора $A_{S}(2)$ на одноименные и перекрестную координатные плоскости КО $O_{s}(2)$.
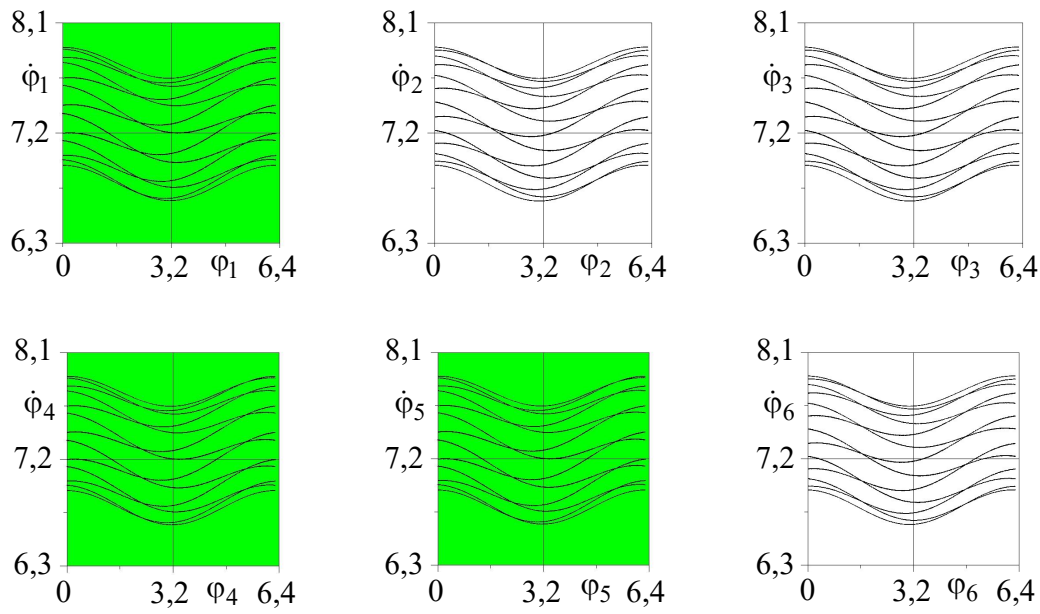

Рис. 6.31. Устойчивая кластерная структура на базе синхронизации трех КО $O_{s}(2)$.
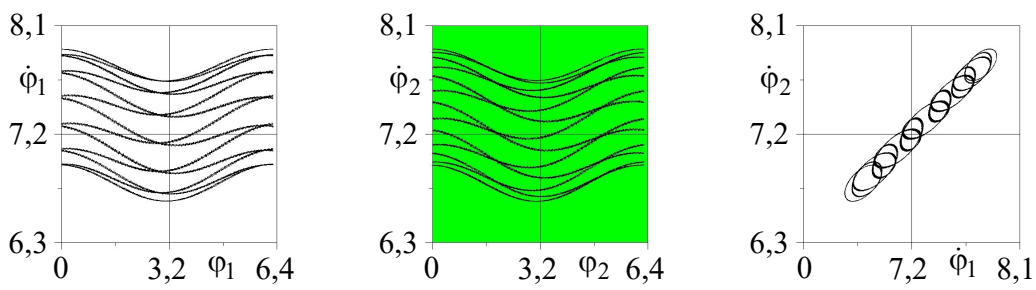

Рис. 6.32. Проекции аттрактора $A_{a}(2)$ на координатные плоскости КО $O_{a}(2)$. Параметры счета: $\lambda=0,07, \gamma=0,51, \sigma=0,079, A=0,28$,

$$
\omega=0,56
$$



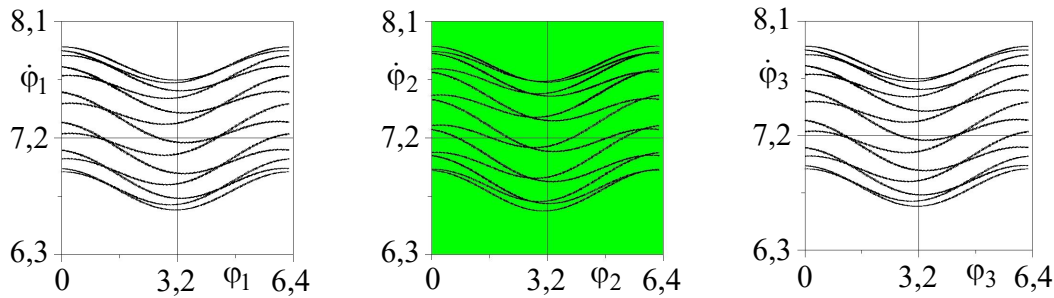

Рис. 6.33. Устойчивая кластерная структура простой клетки $O_{a}(2)$.
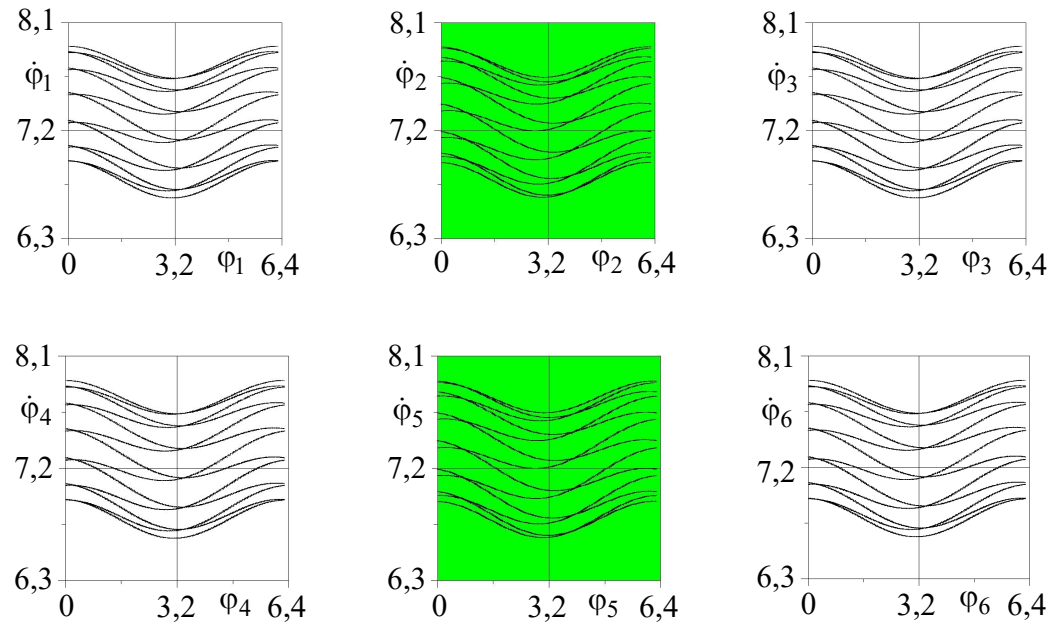

Рис. 6.34. Устойчивая кластерная структура на базе синхронизации двух простых клеток $O_{a}(2)$.

Теперь все кластерные структуры в решетке разделим на типы. Тип совокупности структур будем определять по типу базового КО, определяющего каждую из структур этой совокупности, т. е. число типов структур в решетке является числом всех возможных в данной решетке типов КО.

Теорема 6.1. $O_{s}(n)$ и $O_{a}(n)$ составляют полный набор типов КО в цепочке элементарных осцилляторов с неймановскими граничными условиями. Синхронизация этих КО и их простых клеток определяет все возможные типы существующих в цепочке кластерных структур [67]. 
Доказательство. Для доказательства привлечем радиотехническую схему цепочки осцилляторов (ротаторов), изображенную на рис. 6.35 , а также перепишем уравнения цепочки в общепринятом виде

$$
\dot{\mathbf{X}}_{i}=\mathbf{F}_{i}\left(\mathbf{X}_{i}, t\right)+\varepsilon \mathbf{C}\left(\mathbf{X}_{i-1}-2 \mathbf{X}_{i}+\mathbf{X}_{i+1}\right)
$$

с граничным условием на левом конце $\mathbf{X}_{0}=\mathbf{X}_{1}$. Граничное условие на правом конце будем записывать в порядке доказательства.

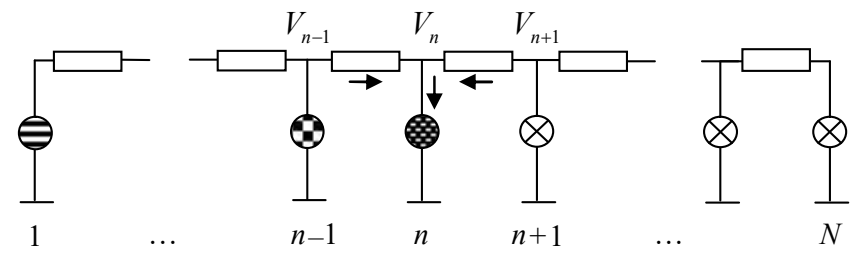

Рис. 6.35. Цепочка осцилляторов (ротаторов).

При доказательстве будем использовать эквивалентные преобразования схем структур в качестве элементарных преобразований данной электрической схемы.

Допустим, что в цепочке реализована некоторая КС. Начиная с первого, выделим все идущие подряд несинхронизованные осцилляторы. Допустим, что их число $n$ (см. рис. 6.35), т. е. $(n+1)$-й элементарный осциллятор синхронизован с одним из осцилляторов выделенной группы. Пусть этот осциллятор синхронизован с некоторым $k$-м, $k \leq n-2$, элементарным осциллятором выделенной группы. В таком случае значения всех одноименных переменных этой пары осцилляторов одинаковы в любой момент времени. Точки на их «входах» - эквипотенциальные, $V_{k}=V_{n+1}$. Проведем эквивалентные схемные преобразования, соответствующие преобразованию схемы кластерной структуры: после замыкания (короткого) эквипотенциальных точек перемычкой выполним последовательность преобразований, как показано на рис. 6.36.

Запишем уравнения для токов синхронизованных осцилляторов в первом и последнем положениях схемы соответственно:

$$
V_{n}-2 V_{n+1}+V_{n+2}=I R, V_{k+1}-2 V_{k}+V_{n+2}=I R \text {. }
$$



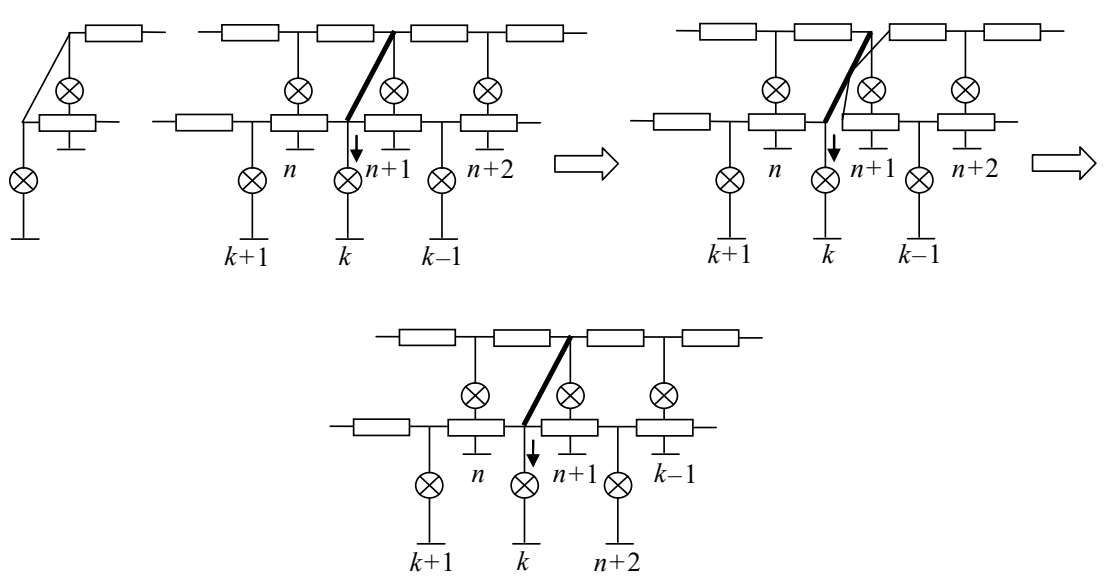

Рис. 6.36. Эквивалентные преобразования цепочки.

Из этих уравнений получаем, что $V_{n}=V_{k+1}$. Кроме случаев $k=n$ и $k=n-1$, это противоречит условию несинхронизованности первых $n$ осцилляторов. Таким образом, $(n+1)$-й осциллятор не может быть синхронизован с любым $k \leq n-2$ осциллятором. Остаются два случая: 1) $k=n, 2) k=n-1$.

1) Допустим, $k=n$, тогда имеем $V_{n}=V_{n+1}$ и $I_{n}=I_{n+1}$. С другой стороны, для этих осцилляторов справедливы уравнения: $V_{n-1}-V_{n}=I_{n} R, V_{n+2}-V_{n+1}=I_{n+1} R$. Отсюда получаем, что $V_{n-1}=V_{n+2}-$ условие синхронизации $(n-1)$-го и $(n+2)$-го осцилляторов. Записывая уравнения Кирхгоффа для этой пары осцилляторов, получаем: $V_{n-2}+V_{n}-2 V_{n-1}=I_{n-1} R, \quad V_{n+3}+V_{n+1}-2 V_{n+2}=$ $=I_{n+2} R$, откуда следует, что $V_{n-2}=V_{n+3}$ и соответствующая пара осцилляторов также синхронизована.

Продолжая последовательную запись уравнений для синхронизованных осцилляторов, получаем $V_{1}=V_{2 n}$. Далее имеем «вилку»: осциллятор с номером $(2 n+1)$ может быть синхронизован с $2 n$-м или с $(2 n-1)$-м. Допустим, что он синхронизован с $(2 n-1)$-м, тогда $V_{2 n+1}=V_{2 n-1}$. Из уравнений для 1 -го и $2 n$-го осцилляторов получаем: $V_{2}-V_{1}=I_{1} R, \quad V_{2 n-1}+V_{2 n}-2 V_{2 n+1}=I_{2 n} R$. Отсюда следует, что 
$V_{2}=V_{1}$, а это противоречит условию отсутствия синхронизации для первых $n$ осцилляторов.

Таким образом, $(2 n+1)$-й осциллятор синхронизован с $2 n$-м. Повторяя рассуждения, получаем порядок следования синхронизованных осцилляторов в цепочке, изображенный на рис. 6.21, структуру на базе $O_{s}(n)$. Покажем, что в этом случае КС содержит целое число изображений КО $O_{s}(n), N=m n$. Допустим обратное: число несинхронизованных ротаторов в конечном отрезке равно $k<n$. Удалим из цепочки все КО (разрежем схему), оставляя предпоследний КО и упомянутый отрезок. Перенумеруем оставшиеся осцилляторы от единицы до $k+n$. В таком случае получаем схему, подобную изображенной на рис. 6.21 , с числом осцилляторов $n+k$. По условию имеем $V_{n+k+1-i}=V_{n-k+i}, i=1,2, \ldots, k$. Запишем уравнения для последнего и $(n-k+1)$-го синхронизованных осцилляторов: $V_{n+k-1}-V_{n+k}=R I, \quad V_{n-k}-2 V_{n-k+1}+V_{n-k+2}=R I, \quad V_{n+k}=V_{n-k+1}$, $V_{n+k-1}=V_{n-k+2}$. Из этих уравнений и условия $V_{n+k}=V_{n-k+1}$, $V_{n+k-1}=V_{n-k+2}$ получаем $V_{n-k}=V_{n-k+1}$. Отсюда следует, что если $k \neq n$, то система первых $n$ ротаторов не является КО, что противоречиво, а если $k=n$, то $V_{0}=V_{1}$, что является граничным условием.

Таким образом, окончательный вывод по этой части доказательства: при условии $\mathbf{X}_{n}=\mathbf{X}_{n+1}$ и несинхронизованности первых $n$ осцилляторов кластерная структура образована на базе синхронизации КО типа $O_{s}(n)$. Учитывая это равенство в системе (6.13), получаем, что она представляет уравнение (6.11) в развернутом виде. Наоборот, сворачивая (6.13), получаем уравнение (6.11) с кластерной матрицей $\mathbf{B}_{s}$.

2) Возвращаясь в начало доказательства, допустим теперь, что $(n+1)$-й осциллятор синхронизован с $(n-1)$-м, т. е. $V_{n-1}=V_{n+1}$. Записывая уравнения Кирхгоффа для этих осцилляторов, получаем: $V_{n-2}-2 V_{n-1}+V_{n}=I R, \quad V_{n+2}-2 V_{n+1}+V_{n}=I R$. Отсюда следует, что $V_{n-2}=V_{n+2}$. Аналогично из этого и всех последующих уравнений получаем общее выражение $V_{i}=V_{2 n-i}, \quad i=1,2, \ldots, n-1$. Вывод: в данном случае динамическая структура системы $(2 n-1)$-х осцил- 
ляторов представляет кластерную структуру простой клетки (см. рис. 6.22). Учитывая условие $\mathbf{X}_{n-1}=\mathbf{X}_{n+1}$ в системе (6.13), получаем, что она представляет собой развернутую запись уравнения КО $O_{a}(n)(6.12)$ с кластерной матрицей $\mathbf{B}_{a}$. Доказательство того, что в данном случае полная схема кластерной структуры цепочки состоит из целого числа схем ПК (см. рис. 6.24), проводится аналогично п. 1.

Окончательный вывод: $O_{s}(n)$ и $O_{a}(n)$ - полный набор типов структурообразующих объектов, которые исчерпывают все типы возможных кластерных структур, которые могут быть реализованы в цепочке осцилляторов.

Замечание. При доказательстве теоремы не использовалось условие однородности цепочки, мы исходили только из того условия, что некая структура в цепочке реализована. Это значит, что теорема имеет место и для неоднородной цепочки. Из доказательства следовало бы, что эта неоднородность должна иметь периодически упорядоченный вид, т. е. для реализации той или иной структуры в цепочке должен быть организован индивидуальный порядок следования неидентичных осцилляторов в соответствии со схемой данной кластерной структуры.

Следствие 1. О числе структур в цепочке при заданном $N$. Если $p$ - количество всех сомножителей (простых и составных) числа $N$, исключая само число и единицу, то в цепочке из $N$ элементарных осцилляторов существует $p$ кластерных структур на базе $O_{s}(n)$ при разных $n$. Если $q$ - количество всех нечетных сомножителей (простых и составных) числа $N$, включая само число, если оно нечетно, то в цепочке существует $q$ кластерных структур на базе ПК КО $O_{a}(n)$ при разных $n$. Полное число кластерных структур в цепочке с заданным $N$ равно $p+q$.

Доказательство. Во-первых, по теореме все КС в цепочке синтезируются или на базе $O_{s}(n)$ или на базе ПК $O_{a}(n)$. Во-вторых, все кластерные структуры состоят из целого числа соответствующих блоков (см. рис. 6.21, 6.24). Для КС на базе $O_{s}(n)$ имеем равенство $N=m n, m \geq 2, n \geq 2$. Отсюда получаем число кластерных структур на его базе, равное $p$. Для структур на базе ПК $O_{a}(n)$ 
$N=m(2 n-1), \quad m \geq 1, n \geq 2$, а это значит, что число таких структур равно $q$. Суммарное число структур равно $p+q$. Для неоднородной цепочки это значит, что такое и только такое число структур можно организовать в цепочке, манипулируя ее неоднородностью.

Пример. Пусть $N=100$; в этом случае $p=7$, а $q=2$, т. е. в цепочке может быть реализовано в сумме девять структур. Полный список этих структур с указанием базы: $O_{s}(2)$ (в структуре содержится $m=50$ штук таких КО), $O_{s}(4)$ (25 шт.), $O_{s}(5)$ (20 шт.), $O_{s}(10)$ (10 шт.), $O_{s}(20)$ (5 шт.), $O_{s}(25)$ (4 шт.), $O_{s}(50)$ (2 шт.), $O_{a}(13)$ (в структуре $m=4$ штуки ПК $\left.O_{a}(13)\right), O_{a}(3)$ (20 шт.).

Следствие 2. Об интегральных многообразиях цепочки осцилляторов как динамической системы. Исходя из арифметических свойств числа осцилляторов в цепочке $N$, а также следствия 1 , можно получить все интегральные многообразия цепочки, представленные в разделе 6.1. Их математические представления получаются приравниванием векторов динамических переменных синхронизованных осцилляторов. По доказанной теореме можно утверждать, что каких-либо других «кластерных» интегральных многообразий, кроме указанных в разделе 6.1 , в цепочке нет.

\section{4. К-осцилляторы кольца и синтез кластерных структур}

Наряду с цепочкой кольцо осцилляторов - одна из базовых моделей теории колебаний и волн [95]. В частности, кольцо является моделью для изучения автоволновых процессов в активных средах с периодическими граничными условиями, исследования динамики коллективных систем фазовой синхронизации, кольцевых систем сверхпроводящих переходов, применяется при моделировании биологических, химических и других систем [57, 73, 75, 96-99 и др.].

В развитие теории кластерных структур ниже мы рассмотрим кластерообразующие объекты кольца и установим все существующие типы кластерных структур [100].

Разрезаемые кластерные структуры кольца. Допустим, что схема кластерной структуры кольца содержит разрезаемую пару. Разрезание этой пары сводит схему в кольце к эквивалентной схеме в цепочке. Это значит, что известные нам базовые КО цепочки 
$O_{s}(n)$ и $O_{a}(n)$ являются базовыми и для кольца. Определения этих КО даны в разделе 6.3 .

Синтез схем разрезаемых кластерных структур осуществляется аналогично цепочке, но с одним замечанием: число схем-блоков КО $O_{s}(n)$ в схеме кластерной структуры всегда четно. Это нетрудно объяснить: во-первых, если в результате разрезания РП схема кольца превращается в схему цепочки, то и наоборот - схема цепочки в результате замыкания конечных осцилляторов превращается в схему кольца. Во-вторых, поскольку в данной процедуре упомянутые осцилляторы являются «одноцветными», то соответствующая структура в цепочке является центрально-симметричной. Отсюда вывод: число схем-блоков $O_{s}(n)$ в соответствующей структуре кольца всегда четно. На рис. 6.37 показана схема разрезаемой кластерной структуры, синтезированная на базе $O_{s}(n)$.

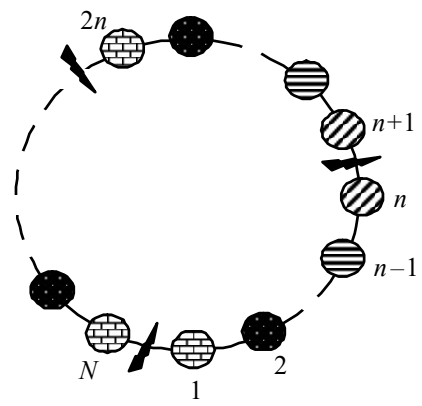

Рис. 6.37. Синтез схемы кластерной структуры кольца на базе КО $O_{s}(n)$.

Для примера на рис. 6.38 представлена разрезаемая кластерная структура как синхронизация пары КО $O_{s}(3)$ в однородном кольце из $N=6$ диффузионно-связанных осцилляторов Чуа. Параметры численного эксперимента $\left\{\alpha ; \beta ; \gamma ; m_{0} ; m_{1} ; \varepsilon\right\}=\{9,5 ; 14$; $0,1 ;-1 / 7 ; 2 / 7 ; 0,34\}$.

Первый и шестой, а также третий и четвертый элементарные осцилляторы - две РП кластерной структуры. 


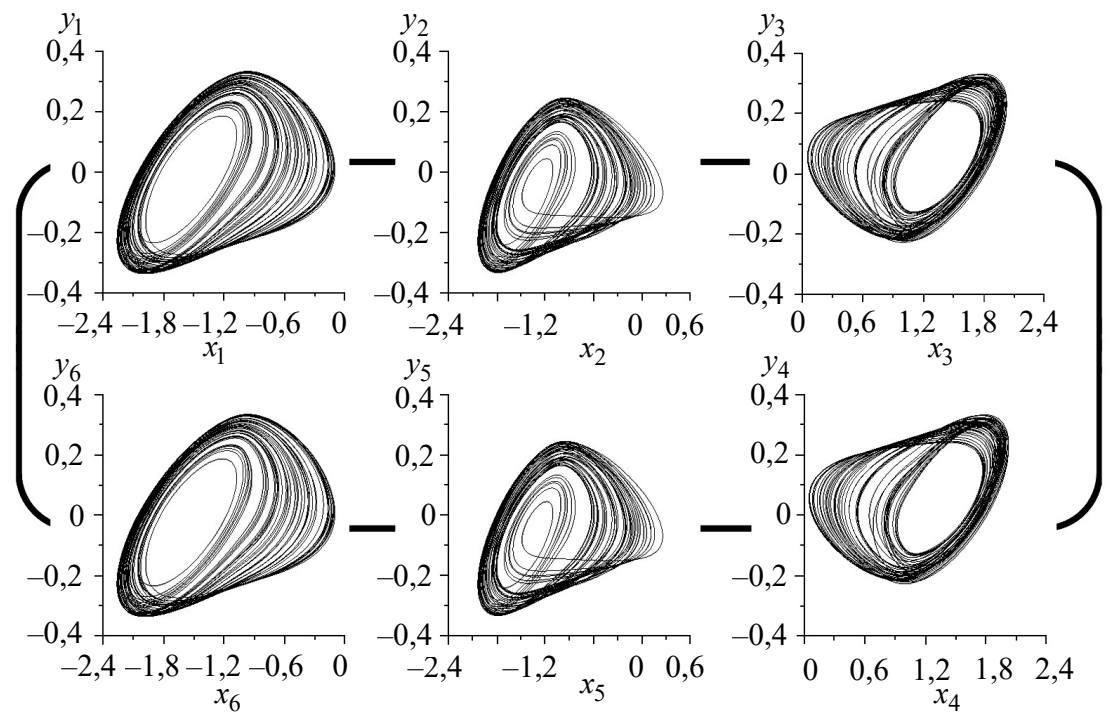

Рис. 6.38. Разрезаемая кластерная структура на базе пары $O_{s}(3)$.

В отличие от структур на базе $O_{s}(n)$ число ПК на базе $O_{a}(n)$ в кластерной структуре кольца может быть любым - как четным, так и нечетным. На рис. 6.39 изображен синтез схемы разрезаемой КС на базе простых клеток $O_{a}(n)$.

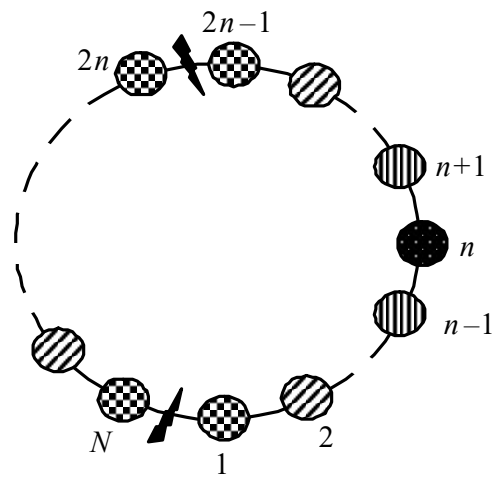

Рис. 6.39. Синтез схемы кластерной структуры в кольце на базе ПК $O_{a}(n)$. 
Для иллюстрации сказанного на рис. 6.40 представлен результат численного эксперимента: показана разрезаемая кластерная структура как синхронизация двух ПК $O_{a}(2)$ в однородном кольце из $N=6$ осцилляторов Чуа. Параметры эксперимента $\left\{\alpha ; \beta ; \gamma ; m_{0} ; m_{1} ; \varepsilon\right\}=\{9,5 ; 14 ; 0,1 ;-1 / 7 ; 2 / 7 ; 0,2175\}$.
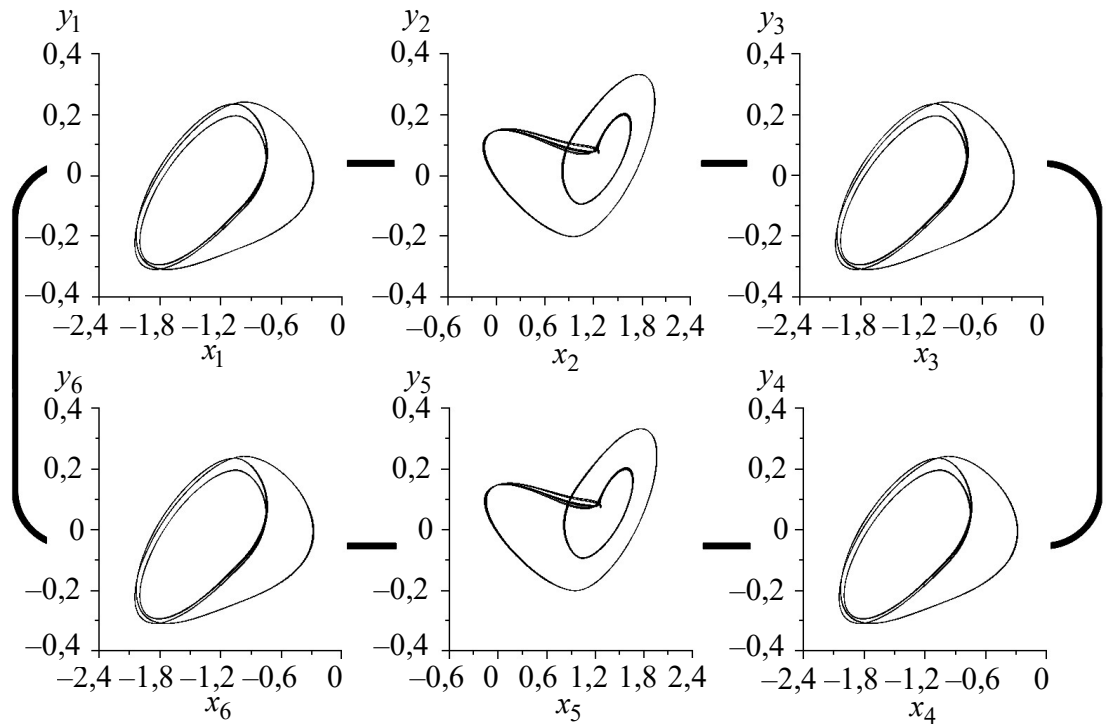

Рис. 6.40. Разрезаемая кластерная структура как синхронизация пары ПК $O_{a}(2)$.

Неразрезаемые кластерные структуры кольия. Предположим, что структура кольца не содержит ни одной РП. В таком случае она (структура) представляет простую клетку, и, следовательно, ее схема должна свертываться к схеме некоторого (искомого) КО, к своей базе. В однородном кольце процедуры свертывания естественно связывать с различными видами его симметрии.

$1^{0}$. Зеркальные кластерные структуры кольца. По опыту с цепочкой нам известна одна из процедур свертывания - перегиб схемы структуры простой клетки через ось симметрии, которую можно рассматривать как след зеркала. В случае кольца эта ось является диаметром, проходящим через середины изображений осцилляторов (считаем кольцо окружностью с равномерным распределением 
осцилляторов). В другом случае оси симметрии - диаметра, проходящего через середины связей, - структура была бы разрезаемой, рассмотрение которой закончено.

Допустим, что схема КС симметрична относительно некоторого диаметра $A F$. Перегибая схему через этот диаметр и совмещая одноцветные изображения, получаем картину, изображенную на рис. 6.41,а. Элементарные осцилляторы располагаются на дуге окружности, причем крайние из них - половинные. Смысл этих половинок нам известен. Если все осцилляторы дуги несинхронизованы (имеют различные цвета), то их система есть искомый КО и операция свертывания закончена.

Однако допустим, что есть общий случай: среди осцилляторов дуги имеются синхронизованные (одноцветные). Тогда схема осцилляторов дуги $A F$ представляет целое число схем КО. Причем схема базового КО должна быть такой, как если бы он занимал всю дугу $A F$ (половинки на концах). В противном случае отсутствовал бы переход на этот случай при повышении числа осцилляторов КО.

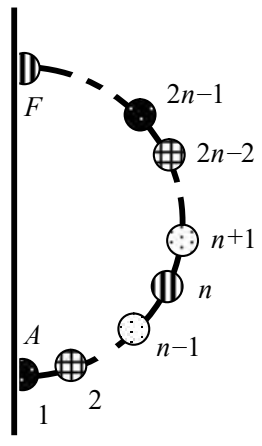

a

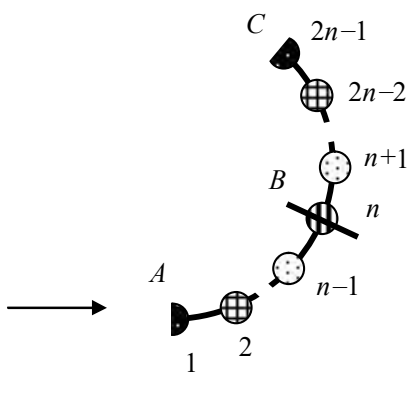

б

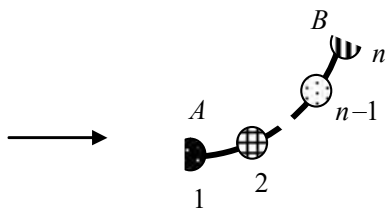

B

Рис. 6.41. Свертывание схемы «зеркальной» КС простой клетки.

Начиная от осциллятора $F$, выделим на схеме все подряд идущие «разноцветные» элементарные осцилляторы - дугу осцилляторов. Продолжая перегибать эту и подобные ей дуги через середину конечного осциллятора, получаем картину, представленную на рис. 6.41,в. На рис. 6.41,б изображена дуга перед последней итерацией. Элементарные осцилляторы на дуге $A F$ представляют схему искомого КО, базового для кластерной структу- 
ры, которую будем называть «зеркальной». Обозначим его $O_{a}^{r}(n)$ и приведем определение.

Определение 6.3. Зеркальным КО $O_{a}^{r}(n)$ будем называть систему $n \geq 2$ связанных элементарных осцилляторов вида

$$
\begin{gathered}
\dot{\mathbf{X}}=\mathbf{G}(\mathbf{X})-\varepsilon \mathbf{B}_{a}^{r} \otimes \mathbf{C X}, \\
\mathbf{B}_{a}^{r}=\left(\mathbf{X}_{1}, \mathbf{X}_{2}, \ldots, \mathbf{X}_{n}\right)^{T}, \mathbf{G}(\mathbf{X})=\left(\mathbf{F}_{1}\left(\mathbf{X}_{1}\right), \mathbf{F}_{2}\left(\mathbf{X}_{2}\right), \ldots, \mathbf{F}_{n}\left(\mathbf{X}_{n}\right)\right)^{T}, \\
\left.\begin{array}{rrrrrrrrrrr}
2 & -2 & 0 & 0 & & & \\
-1 & 2 & -1 & 0 & & & \\
0 & -1 & 2 & -1 & & & & \\
0 & 0 & -1 & 2 & & & & & \\
& & & & \ddots & & & & \\
& & & & & & & & -1 & 0 & 0 \\
& 0 & & & & -1 & 2 & -1 & 0 \\
& & & & & 0 & -1 & 2 & -1 \\
& & & & 0 & -2 & 2
\end{array}\right)
\end{gathered}
$$

при условии существования в фазовом пространстве системы аттрактора $A_{a}^{r}(n)$ такого, что для $\forall\left(\boldsymbol{X}_{1}, \boldsymbol{X}_{2}, \ldots, \boldsymbol{X}_{n}\right) \in A_{a}^{r}(n)$, $\mathbf{X}_{i} \neq \mathbf{X}_{j}, \quad i \neq j, \quad i=\overline{1, n}, \quad j=\overline{1, n}$.

Уравнение (6.14) можно получить разными способами, и в частности из уравнения (6.13), где в качестве граничных условий следует взять $\mathbf{X}_{2} \equiv \mathbf{X}_{0}, \mathbf{X}_{n+1} \equiv \mathbf{X}_{n-1}$.

Для иллюстрации на рис. 6.42 изображена зеркальная кластерная структура в однородном кольце из $N=6$ осцилляторов Чуа. Параметры эксперимента $\left\{\alpha ; \beta ; \gamma ; m_{0} ; m_{1} ; \varepsilon\right\}=\{9,11 ; 14 ; 0,1 ;-1 / 7 ; 2 / 7 ; 0,25\}$. Плоскость «зеркала» проходит через первый и четвертый осцилляторы.

Синтез схемы зеркальной структуры ведется в порядке, обратном свертыванию, - цепочкой зеркальных отражений схемы «зеркального» КО: к дуге осцилляторов $A B$ присоединяется ее зеркаль- 
ное отражение и т. д. вплоть до замыкания схемы структуры в кольцо.

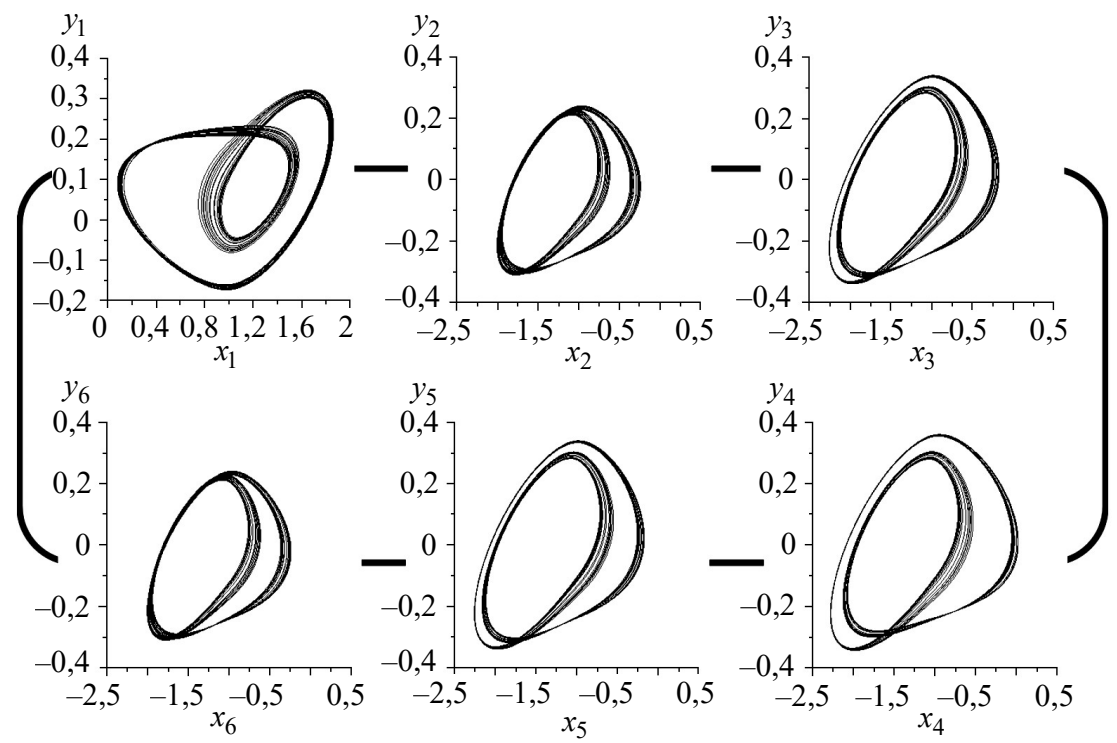

Рис. 6.42. Зеркальная кластерная структура на базе $O_{a}^{r}(4)$.

Нетрудно вывести формулу для связи числа осцилляторов в кольце с числом КО в синтезе структуры и числом элементарных осцилляторов в самом КО: $N=2 m(n-1)$, где $m$ - число КО, «покрывающих» половину кольца. Из этой формулы заключаем, что если $r$ - число всех сомножителей числа $N / 2$, включая само число, то в кольце существует $r$ зеркальных кластерных структур на базе КО $O_{a}^{r}(n)$. Например, в случае $N=12$ существуют три структуры на базе $O_{a}^{r}(n)$ : структура из двух $O_{a}^{r}(7)$, из четырех $O_{a}^{r}(4)$ и шести КО $O_{a}^{r}(3)$. В случае $N=6$ в кольце существует одна зеркальная структура на базе синхронизации $O_{a}^{r}(4)$, пример которой изображен на рис. 6.42 .

$2^{0}$. Циклические кластерные структуры кольца. Этот тип кластерных структур связан с симметрией поворота кольца. Однако 
мы подойдем к существованию таких структур и определим их базовый КО исходя из физических принципов, отраженных в элементарных преобразованиях и свертываемости схем структур.

Допустим, что в кольце реализована некоторая неразрезаемая $n$-кластерная структура. Сведем все элементарные осцилляторы какого-нибудь одного кластера в один узел (короткие замыкания) (рис. $6.43, a)$. В результате получим фигуру, напоминающую $m$-лепестковую «розу» (рис. 6.43,б). Допустим, что среди осцилляторов каждого лепестка нет одноцветных (из разных кластеров). Теперь докажем, что в этом случае порядок следования осцилляторов на лепестках одинаков: продолжим свертывание схемы КС, поворачивая «лепестки» до совмещения одноцветных осцилляторов (короткие замыкания); в результате образуется кольцо несинхронизованных осцилляторов (рис. 6.43,в). Это кольцо и является искомым КО «циклических» структур.

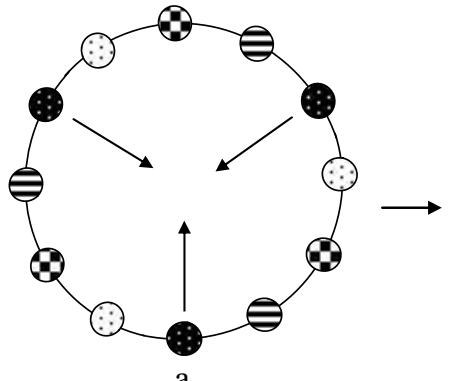

a

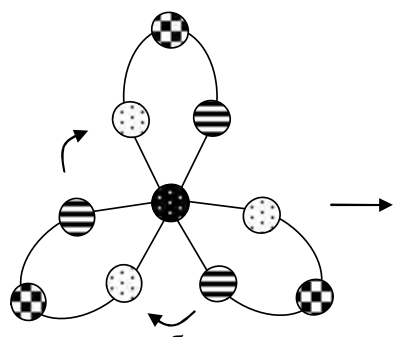

6

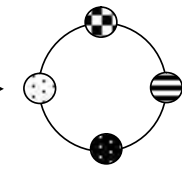

B

Рис. 6.43. Свертывание схемы циклической структуры.

Синтез схемы циклической структуры ведется в обратном порядке: считаем, что имеется $m$ идентичных колец (КО), наложенных друг на друга. Далее эта система разворачивается относительно изображения любого осциллятора (точнее, узла из $m$ одинаковых осцилляторов) в $m$-лепестковую «розу». Затем изображения осцилляторов узла распускаются по своим местам. Тот же результат получаем в результате последовательных поворотов дуги «разноцветных» осцилляторов.

Обозначим базовый КО циклических КС $O_{s}^{r}(n)$ и приведем его определение. 
Определение 6.4. Циклическим КО $O_{s}^{r}(n)$ будем называть систему $n \geq 3$ связанных элементарных осцилляторов вида

$$
\begin{gathered}
\dot{\mathbf{X}}=\mathbf{G}(\mathbf{X})-\varepsilon \mathbf{B}_{s}^{r} \otimes \mathbf{C X}, \\
\mathbf{X}=\left(\mathbf{X}_{1}, \mathbf{X}_{2}, \ldots, \mathbf{X}_{n}\right)^{T}, \quad \mathbf{G}(\mathbf{X})=\left(\mathbf{F}_{1}\left(\mathbf{X}_{1}\right), \mathbf{F}_{2}\left(\mathbf{X}_{2}\right), \ldots, \mathbf{F}_{n}\left(\mathbf{X}_{n}\right)\right)^{T}, \\
\mathbf{B}_{s}^{r}=\left(\begin{array}{cccccccccc}
2 & -1 & 0 & 0 & \ldots & 0 & 0 & 0 & -1 \\
-1 & 2 & -1 & 0 & \ldots & 0 & 0 & 0 & 0 \\
0 & -1 & 2 & -1 & \ldots & 0 & 0 & 0 & 0 \\
0 & 0 & -1 & 2 & \ldots & 0 & 0 & 0 & 0 \\
\vdots & \vdots & \vdots & \vdots & \ddots & \vdots & \vdots & \vdots & \vdots \\
0 & 0 & 0 & 0 & \ldots & 2 & -1 & 0 & 0 \\
0 & 0 & 0 & 0 & \ldots & -1 & 2 & -1 & 0 \\
0 & 0 & 0 & 0 & \ldots & 0 & -1 & 2 & -1 \\
-1 & 0 & 0 & 0 & \ldots & 0 & 0 & -1 & 2
\end{array}\right)
\end{gathered}
$$

при условии существования в фазовом пространстве системы аттрактора $A_{s}^{r}(n)$ такого, что для $\forall\left(\mathbf{X}_{1}, \mathbf{X}_{2}, \ldots, \mathbf{X}_{n}\right) \in A_{s}^{r}(n)$, $\mathbf{X}_{i} \neq \mathbf{X}_{j}, \quad i \neq j, \quad i=\overline{1, n}, \quad j=\overline{1, n}$.

Нетрудно убедится, что уравнение (6.15) представляет кольцо из $n$ осцилляторов.

Для иллюстрации на рис. 6.44 представлена циклическая кластерная структура, полученная в однородном кольце из $N=6$ осцилляторов Чуа. Параметры эксперимента $\left\{\alpha ; \beta ; \gamma ; m_{0} ; m_{1} ; \varepsilon\right\}=$ $=\{9,5 ; 14 ; 0,1 ;-1 / 7 ; 2 / 7 ; 0,08\}$. Очевидно, что число синхронизованных КО и число кластеров связано с числом осцилляторов в кольце простым соотношением $N=m n$. Это позволяет сформулировать столь же простое утверждение: пусть $s$ - число всех сомножителей числа $N$, исключая само $N$, тогда число циклических структур в кольце равно $s$. Например, в случае $N=12$ существуют четыре циклические структуры на базе $O_{s}^{r}(n)$ : структура из двух $O_{s}^{r}(6)$, из трех $O_{s}^{r}(4)$, из четырех $O_{s}^{r}(3)$ и шести КО $O_{s}^{r}(2)$. 
В случае $N=6$ существуют две циклические кластерные структуры, одна из которых (трехкластерная) изображена на рис. 6.44.

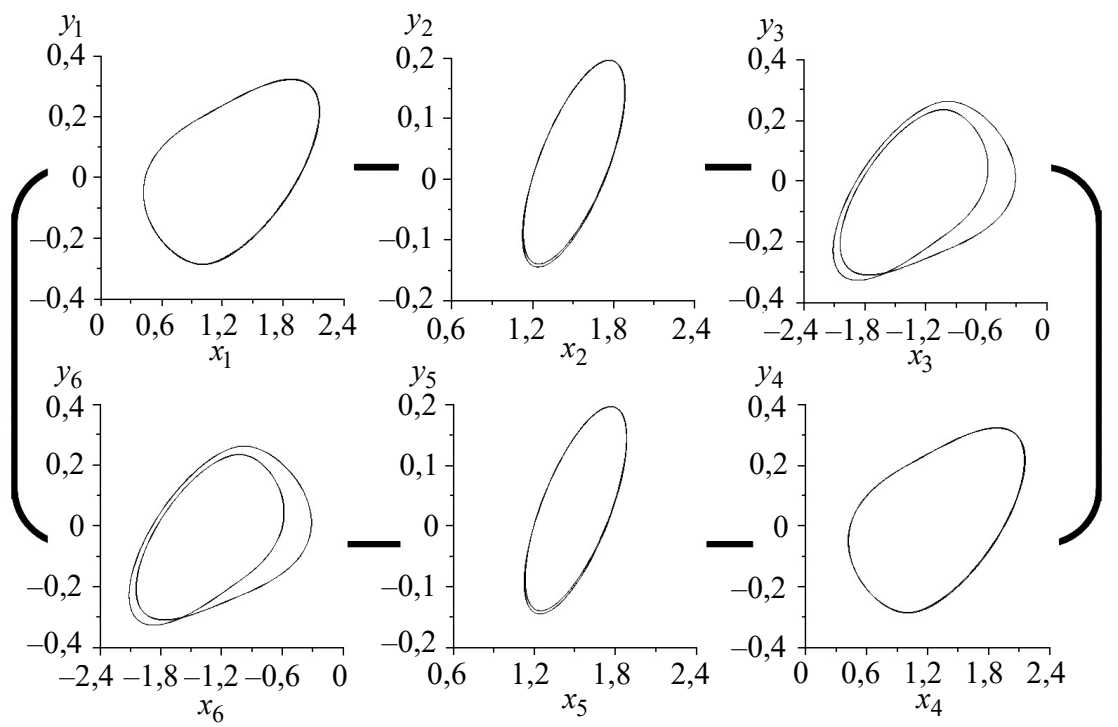

Рис. 6.44. Циклическая кластерная структура на базе $O_{s}^{r}(3)$.

Замечание. Кластерные структуры, синтезированные в кольце на базе зеркального КО с двумя элементарными осцилляторами, наряду с зеркальной имеют и циклическую симметрию (рис. 6.45).

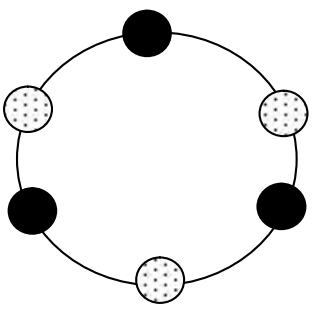

a

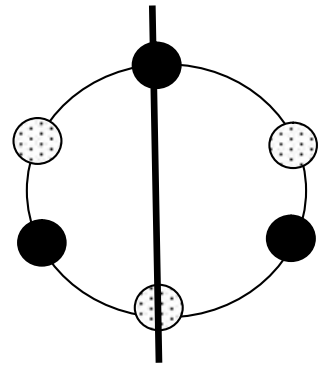

6

Рис. 6.45. Структура с двойной симметрией. 
Двойственность в вопросе, к какому типу отнести эту структуру, снимается, если записать уравнения кольца в виде системы связанных КО. При этом обнаружится, что структура образована на базе зеркального КО.

Выше определены четыре типа КС кольца. Внутреннее убеждение (все учтено, ничего другого быть не может) подсказывает, что найдена полная группа КО. Попробуем доказать это. Предварим доказательство простой леммой.

Лемма 6.1.

1) Если осцилляторы на концах двух параллельных хорд попарно синхронизованы (рис. 6.46,a), то попарно синхронизованы осцилляторы на концах всех хорд связки.

2) Если осцилляторы на концах двух пересекающихся хорд попарно синхронизованы (рис. 6.46,б), то попарно синхронизованы осцилляторы на концах всех хорд пучка (осцилляторы распределены по кольцу соответствующим образом).

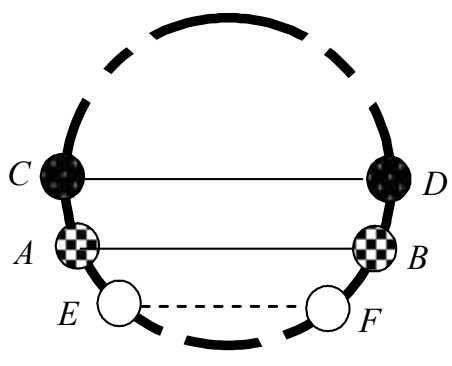

a

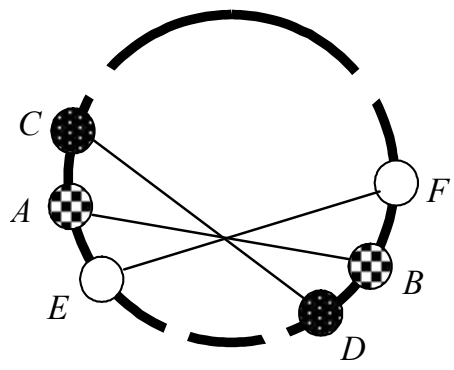

6

Рис. 6.46. К лемме 6.1.

Доказательство.

1) Рассматриваем осцилляторы хорды $E F$. Напишем уравнения Кирхгоффа для осцилляторов $A$ и $B$ :

$$
V_{C}+V_{E}-2 V_{A}=I_{A} R, \quad V_{D}+V_{F}-2 V_{B}=I_{B} R .
$$

По условию $V_{A}=V_{B}, V_{C}=V_{D}, I_{A}=I_{B}$ (синхронизация). Отсюда получаем равенство $V_{E}=V_{F}$, т. е. осцилляторы $E$ и $F$ являются синхронизованными. Далее рассуждения повторяются для осцилляторов всех остальных хорд связки. 
2) Доказательство аналогично первому случаю. Рассмотрим осцилляторы хорды $E F$. Уравнения Кирхгоффа для осцилляторов $A$ и $B$ :

$$
V_{C}+V_{E}-2 V_{A}=I_{A} R, \quad V_{D}+V_{F}-2 V_{B}=I_{B} R .
$$

По условию $V_{A}=V_{B}, V_{C}=V_{D}, I_{A}=I_{B}$ (синхронизация). Отсюда получаем равенство $V_{E}=V_{F}$, т. е. осцилляторы $E$ и $F$ являются синхронизованными. Далее рассуждения повторяются для следующей и всех остальных хорд пучка.

Теорема 6.2. КО $O_{s}(n), O_{a}(n), O_{a}^{r}(n)$ и $O_{s}^{r}(n)$ составляют полный набор типов КО в кольце осцилляторов и определяют все типы существующих в кольце кластерных структур.

Доказательство. Допустим, что в кольце реализована некоторая кластерная структура. Выделим дугу $A F$ с максимальным числом $n$ несинхронизованных осцилляторов и пронумеруем их, начиная с одного из концов, против часовой стрелки (рис. 6.47).

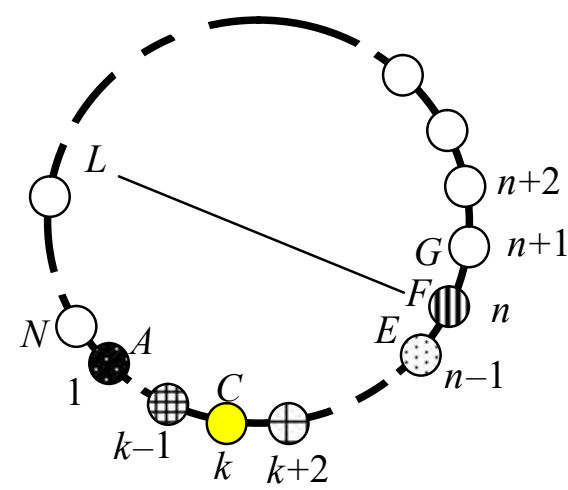

Рис. 6.47. К доказательству теоремы 6.2 .

По условию осциллятор $G$ синхронизован с одним из осцилляторов дуги $A F$ Будем рассматривать различные случаи. Если в каждом из них кластерную структуру будет представлять один из четверки указанных КО, то это будет доказательством теоремы, исключая, разумеется, физически невозможные случаи. 
1) Допустим, что осциллятор $G$ синхронизован с осциллятором $F$. В таком случае эта пара осцилляторов - разрезаемая и данная КС является разрезаемой. Базовые КО такой структуры $O_{s}(n)$ или $O_{a}(n)$.

2) Допустим, что осциллятор $G$ синхронизован с осциллятором $E$. По лемме 1 (частный случай - одна из хорд стянута в точку) осцилляторы на концах всех хорд, параллельных $G E$, синхронизованы, и мы имеем структуру, симметричную относительно диаметpa, проходящего через осциллятор $F$. Если число осцилляторов в кольце нечетно, то последняя из связки короткая хорда стягивает разрезаемую пару, т. е. опять имеется разрезаемая структура с базовым КО $O_{a}(n)$. Если число осцилляторов четно, то диаметр $F L$ проходит через середины осцилляторов. Допустим, что среди осцилляторов дуги $L G$ нет РП (в противном случае структура является разрезаемой и ее КО $\left.O_{a}(n)\right)$. В таком случае эта кластерная структура (симметричная относительно диаметра $F L$ ) является «зеркальной» и ее КО $O_{a}^{r}(n)$.

3) Допустим, что осциллятор $G$ синхронизован с произвольным осциллятором $C$. Производим эквивалентные преобразования КС: синхронизованные осцилляторы $C$ и $G$ «стягиваем» в узел, а далее «разводим» их по разным окружностям (рис. $6.48, a, б)$.

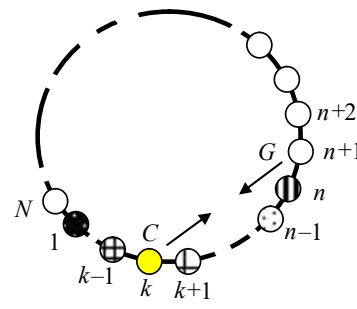

a

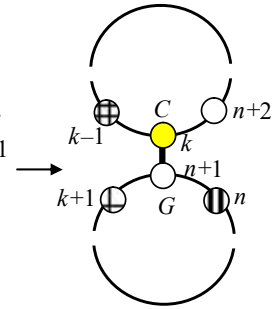

6

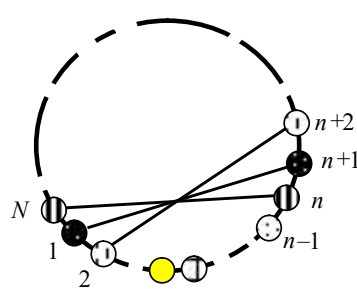

B

Рис. 6.48. Эквивалентные преобразования схемы КС.

Запишем уравнения Кирхгоффа для осцилляторов $G$ и $C$ в первом и втором положениях системы: 


$$
\begin{aligned}
& V_{n+2}+V_{n}-2 V_{G}=I_{G} R, \quad V_{k-1}+V_{k+2}-2 V_{C}=I_{C} R, \\
& V_{k-1}+V_{n+2}-2 V_{C}=I_{C} R, \quad V_{k+1}+V_{n}-2 V_{G}=I_{G} R .
\end{aligned}
$$

При учете равенств $V_{G}=V_{C}, I_{G}=I_{C}$ (синхронизация) получаем $V_{n}=V_{k-1}$ и $V_{n+2}=V_{k+1}$. При всех $k>1$ эти уравнения противоречат условию, что первые $n$ осцилляторов несинхронизованы, а при $k=1$ с учетом того, что $V_{0}=V_{N}$, они дают равенства $V_{n}=V_{N}$, $V_{n+2}=V_{2}$. По лемме 6.1 последние уравнения определяют циклическую кластерную структуру с базовым КО $O_{s}^{r}(n)$ (рис. 6.48,в). Теорема доказана.

Следствие. Если $p$ - количество всех сомножителей (простых и составных) числа $N / 2$, включая само число, а $q$ - количество всех нечетных сомножителей числа $N$, включая его, если оно нечетно, то полное число разрезаемых структур в кольце равно $p+q$. Если $r$ - число всех сомножителей числа $N / 2$, включая само число, то в кольце существует $r$ «зеркальных» КС на базе КО $O_{a}^{r}(n)$. Если $s$ - число всех сомножителей числа $N$, исключая само $N$, то число циклических структур на базе $O_{s}^{r}(n)$ равно $s$. Полное число КС всех типов равно $p+q+r+s$.

Доказательство следствия фактически дано выше, при исследовании типов кластерных осцилляторов.

Например, если $N=12$, то, по следствию, в кольце существует 11 кластерных структур. Их перечисление не составляет труда. Заметим, что в случае простого $N$ в кольце существует единственная (она же разрезаемая) структура на базе ПК $O_{a}(n)$ с числом кластеров $n=(N+1) / 2$.

В завершение раздела заметим также, что указанные выше типы КО могут быть в основе схем структур не только собственно в кольце, но и в других решетках, для которых кольцо является элементом конструкции (рис. 6.49). 


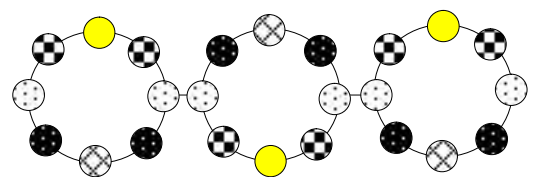

a

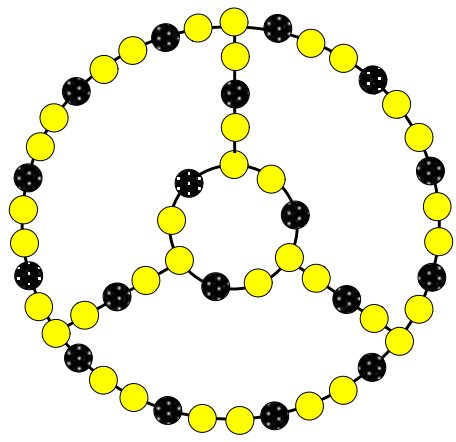

B

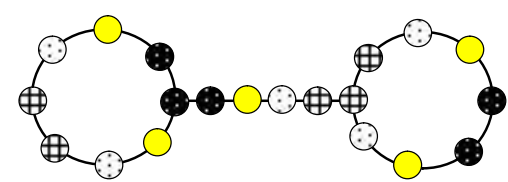

6

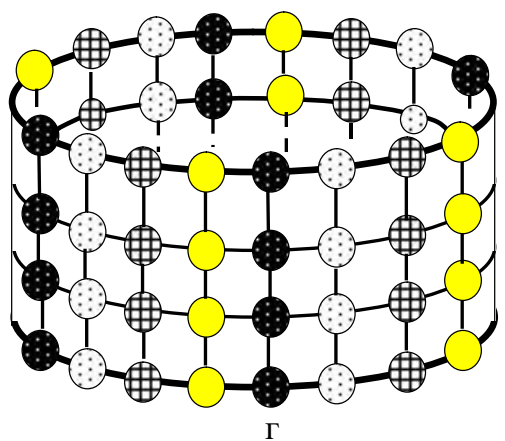

Рис. 6.49. Примеры кластерных структур на базе различных КО кольца:

а - структура на базе КО $O_{a}^{r}(5), \sigma-O_{s}(4)$, в - $O_{a}(2), \Gamma-O_{s}^{r}(4)$.

\section{5. Устойчивость кластерных структур}

Фазовое пространство однородных решеток осцилляторов устроено сложно. В общем случае наряду с аттрактором, представляющим интересующую нас кластерную структуру (например, $A_{s}(n), A_{a}(n)$ и др.), в фазовом пространстве существуют и другие аттракторы. В их числе могут быть аттракторы побочных кластерных структур, в также аттрактор $A(1)$, соответствующий пространственно однородному состоянию решетки, который присутствует всегда. Это обстоятельство существенно усложняет проблему исследования устойчивости структур, в том числе сужая набор методов исследования (исключается второй метод Ляпунова). Кроме того, если внутренние свойства «главного» аттрактора $A(1)$, с которым мы имели дело ранее, не зависели от параметра решетки $\varepsilon$, то этого нельзя сказать о кластерных аттракторах. Их свойства, в частности ляпуновские показатели, так же, как и само существо- 
вание названных аттракторов, определяются данным параметром, что исключает возможность формулировок условий устойчивости в явном виде, подобно случаю пространственно однородных структур. Это создает дополнительные проблемы по уточнению получаемых аналитических условий устойчивости и предполагает дополнительные численные или натурные исследования.

Далее предполагаем, что аттракторы интересующих нас кластерных структур существуют и свойства их известны; в частности, считаем известными максимальные ляпуновские показатели аттракторов.

1) Устойчивость простейшей кластерной структуры на базе $O_{s}(n)$ при параллельном синтезе.

На рис. 6.50 приведена схема К-осциллятора $O_{s}(n)$ и схема ленточной кластерной структуры на его базе.

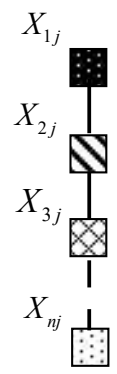

a

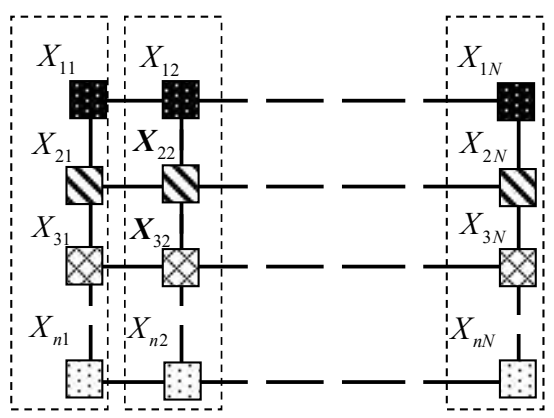

6

Рис. 6.50. Схема КО $O_{s}(n)(\mathrm{a})$ и ленточной кластерной структуры на его базе (б).

Уравнения решетки составляем, используя опыт предыдущих разделов. Для векторов динамического состояния КО $\mathbf{Y}_{j}=\operatorname{col}\left(\mathbf{X}_{1 j}, \mathbf{X}_{2 j}, \ldots, \mathbf{X}_{n j}\right), j=\overline{1, N_{s}}$ эти уравнения имеют вид

$$
\begin{aligned}
& \dot{\mathbf{Y}}_{j}=\mathbf{G}\left(\mathbf{Y}_{j}\right)-\varepsilon\left(\mathbf{I}_{n} \otimes \mathbf{C}\right)\left(-\mathbf{Y}_{j-1}+2 \mathbf{Y}_{j}-\mathbf{Y}_{j+1}\right), \\
& j=1,2, \ldots N .
\end{aligned}
$$


Граничные условия: $\mathbf{Y}_{0}=\mathbf{Y}_{1}, \mathbf{Y}_{N_{s}}=\mathbf{Y}_{N_{s}+1}$.

Исследуем локальную устойчивость аттрактора $A_{s}(n)$, линеаризуя (6.16) в окрестности решения $\mathbf{Y}_{1}=\mathbf{Y}_{2}=\ldots=\mathbf{Y}_{N_{s}}=\xi(t) \in A_{s}(n)$.

Относительно вектора $\mathbf{U}=\left(\mathbf{U}_{1}, \mathbf{U}_{2}, \ldots, \mathbf{U}_{N_{s}-1}\right)^{T}$, где $\mathbf{U}_{i}=\mathbf{Y}_{i}-\mathbf{Y}_{i+1}$, соответствующая линейная система, записанная в виде одного уравнения, имеет вид

$$
\dot{\mathbf{U}}=\left(\mathbf{I}_{p} \otimes \mathbf{J}(\xi)-\varepsilon \mathbf{D}_{p} \otimes \mathbf{I}_{n} \otimes \mathbf{C}\right) \mathbf{U},
$$

где $\quad p=N_{s}-1, \quad \mathbf{J}(\xi)=\operatorname{diag}\left(\mathbf{J}_{1}\left(\xi_{1}\right), \mathbf{J}_{2}\left(\xi_{2}\right), \ldots, \mathbf{J}_{n}\left(\xi_{n}\right)\right)-\varepsilon \mathbf{B}_{n} \otimes \mathbf{C}-$ матрица Якоби КО $O_{s}(n), \mathbf{J}_{k}\left(\xi_{k}\right)$ - матрица Якоби элементарного осциллятора, $\mathbf{D}_{p}$ - матрица вида

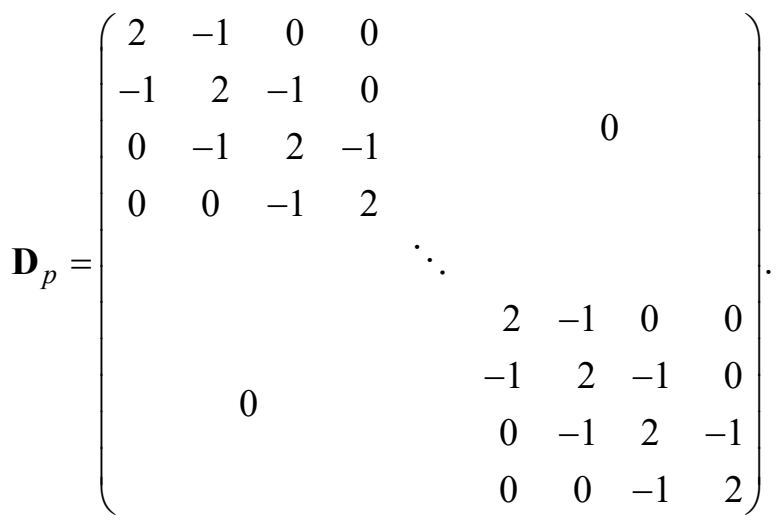

Уравнение (6.17) подпадает под лемму 5.1 и, соответственно, под теорему 5.1 при условии замены матрицы С (как в лемме и теореме) на матрицу $\mathbf{I}_{n} \otimes \mathbf{C}$. Учитывая этот факт и то, что собственные значения матрицы $\mathbf{D}_{p}$ нам известны, получаем условия устойчивости кластерной структуры в виде следующих неравенств: $\varepsilon>\lambda_{s}(n) / 4 \sin ^{2} \frac{\pi}{2 N_{s}}$, если $\mathbf{C}=\mathbf{I}_{m}, \quad$ и $\varepsilon>\varepsilon^{*}\left(\lambda_{s}(n)\right) / 4 \sin ^{2} \frac{\pi}{2 N_{s}}-$ в общем случае матрицы $\mathbf{C}$. 
Как видим, полученные неравенства для устойчивости данной кластерной структуры и соответствующие неравенства в случае пространственно однородной структуры в цепочке по форме одинаковы: схему на рис. 6.51 можно интерпретировать как «цепочку цепочек», поэтому число элементарных осцилляторов заменяется числом блоков - КО. Принципиальное отличие состоит в том, что в данном случае ляпуновский показатель зависит от числа $\varepsilon$ : $\lambda_{s}=\lambda_{s}(n, \varepsilon)$. То есть по параметру связи данные условия представлены неявно, а значит, для полноты картины требуются дополнительные численные или натурные исследования зависимости показателя от этого параметра. В [93] даны схемы, математическое обоснование и описана процедура измерения показателей в натурном эксперименте. Это касается случая хаотических кластерных аттракторов. В случае же регулярных аттракторов ситуация выглядит просто: если кластерный аттрактор является регулярным, а значит, $\lambda_{s}(n, \varepsilon) \leq 0$, то структура является устойчивой при всех соответствующих значениях $\varepsilon$, в том числе и при сколь угодно малых.

2) Устойчивость кластерной структуры на базе $O_{s}(n)$ при последовательном синтезе.

Для начала исследуем случай двух КО (рис. 6.51).

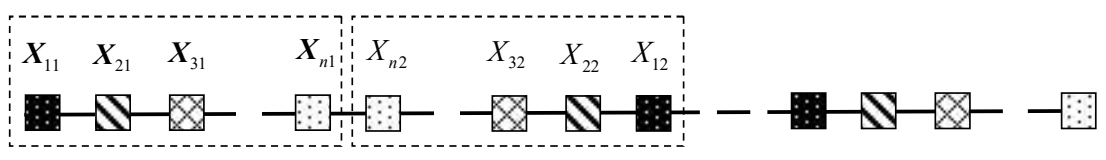

Рис. 6.51. КС на базе $O_{s}(n)$

Относительно векторов $\mathbf{Y}_{j}=\operatorname{col}\left(\mathbf{X}_{1 j}, \mathbf{X}_{2 j}, \ldots, \mathbf{X}_{n j}\right), j=1,2$ динамическая система имеет следующий вид:

$$
\begin{aligned}
& \dot{\mathbf{Y}}_{1}=\mathbf{G}\left(\mathbf{Y}_{1}\right)-\varepsilon\left(\mathbf{C}_{*} \otimes \mathbf{C}\right)\left(\mathbf{Y}_{1}-\mathbf{Y}_{2}\right), \\
& \dot{\mathbf{Y}}_{2}=\mathbf{G}\left(\mathbf{Y}_{2}\right)+\varepsilon\left(\mathbf{C}_{*} \otimes \mathbf{C}\right)\left(\mathbf{Y}_{1}-\mathbf{Y}_{2}\right),
\end{aligned}
$$

где $\mathbf{C}_{*}=\operatorname{diag}(0,0, \ldots, 0,1)-$ матрица синтеза. Линеаризация (6.18) по переменной $\mathbf{U}=\mathbf{Y}_{1}-\mathbf{Y}_{2}$ приводит к уравнению

$$
\dot{\mathbf{U}}=\left(\mathbf{J}(\xi)-\varepsilon 2 \mathbf{C}_{*} \otimes \mathbf{C}\right) \mathbf{U} .
$$


Непосредственно из уравнения (6.19) получаем: структура с регулярной внутренней динамикой устойчива. Это следует из того, что матрица $\varepsilon 2 \mathbf{C}_{*} \otimes \mathbf{C}$ является диагональной и ее можно рассматривать в виде возмущения якобиана $\mathbf{J}(\xi)$. Подобные возмущения не увеличивают показатели решений $[101,102]$. В отличие от параллельного соединения, где матрицей синтеза являлась единичная матрица $\left(\mathbf{C}_{*} \rightarrow \mathbf{I}_{n}\right)$, в данном случае сформулировать условия устойчивости непосредственно из уравнения (6.19) нельзя или, по крайней мере, затруднительно. Для преодоления технических трудностей сформулируем дополнительную лемму.

Лемма 6.2. Допустим, что норма переменной матрицы $A(t)$ ограничена: $\|A(t)\|=\mu$, а $\lambda_{\min }>0$ - наименьший корень постоянной симметрической матрицы $B$. Тогда при $\varepsilon>\mu / \lambda_{\min }$ тривиальное решение уравнения

$$
\dot{\mathbf{y}}=(\mathbf{A}(t)-\varepsilon \mathbf{B}) \mathbf{y}
$$

асимптотически устойчиво.

Доказательство. Допустим, что $\mathbf{Y}\left(t, t_{0}\right)$ - матрицант уравнения $\dot{\mathbf{y}}=-\varepsilon \mathbf{B y}$. Тогда $\mathbf{Y}\left(t, t_{0}\right)=e^{-\varepsilon B\left(t-t_{0}\right)}$ и $\left\|\mathbf{Y}\left(t, t_{0}\right)\right\|=e^{-\varepsilon \lambda_{\min }\left(t-t_{0}\right)}$. Принимая выражение $\mathbf{A}(t) \mathbf{y}$ в качестве внешнего возмущения, запишем (6.20) в интегральной форме:

$$
\mathbf{y}(t)=\int_{t_{0}}^{t} \mathbf{Y}(t, \tau) \mathbf{A}(\tau) \mathbf{y}(\tau) d \tau+\mathbf{Y}\left(t, t_{0}\right) \mathbf{c},
$$

где с - произвольный постоянный вектор. Оценивая норму правой и левой частей этого уравнения, получаем неравенство

$$
\|\mathbf{y}(t)\| \leq \int_{t_{0}}^{t}\|\mathbf{Y}(t, \tau)\|\|\mathbf{A}(\tau)\|\|\mathbf{y}(t)\| d \tau+\left\|\mathbf{Y}\left(t, t_{0}\right)\right\|\|\mathbf{c}\|,
$$

которое с учетом предложенных условий преобразуется к виду

$$
\|\mathbf{y}(t)\| e^{\varepsilon \lambda_{\min } t} \leq \int_{t_{0}}^{t} \mu\|\mathbf{y}(\tau)\| e^{\varepsilon \lambda_{\min } \tau} d \tau+C e^{\varepsilon \lambda_{\min } t_{0}},
$$

где $C$ - произвольная постоянная. 
После применения к данному неравенству известной леммы (об интегральном неравенстве) [103], и дальнейших незначительных преобразований получаем, что $\|\mathbf{y}(t)\| \leq C e^{-\left(\varepsilon \lambda_{\min }-\mu\right)\left(t-t_{0}\right)}$. Из этого неравенства следует: если $\varepsilon>\mu / \lambda_{\min }$, то $\mathbf{y}(t) \rightarrow 0$ при $t \rightarrow \infty$. Лемма доказана.

Установим связь уравнений (6.19) и (6.20). Для этого в якобиане КО $\mathbf{J}(\xi)=\operatorname{diag}\left(\mathbf{J}_{1}\left(\xi_{1}\right), \mathbf{J}_{2}\left(\xi_{2}\right), \ldots, \mathbf{J}_{n}\left(\xi_{n}\right)\right)-\varepsilon \mathbf{B}_{n} \otimes \mathbf{C}$ выделим переменную часть $\mathbf{A}(t)=\operatorname{diag}\left(\mathbf{J}_{1}\left(\xi_{1}\right), \mathbf{J}_{2}\left(\xi_{2}\right), \ldots, \mathbf{J}_{n}\left(\xi_{n}\right)\right)$ (укороченный якобиан), а кластерную матрицу $\mathbf{B}_{n}$ объединим с матрицей $2 \mathbf{C}_{*}$ :

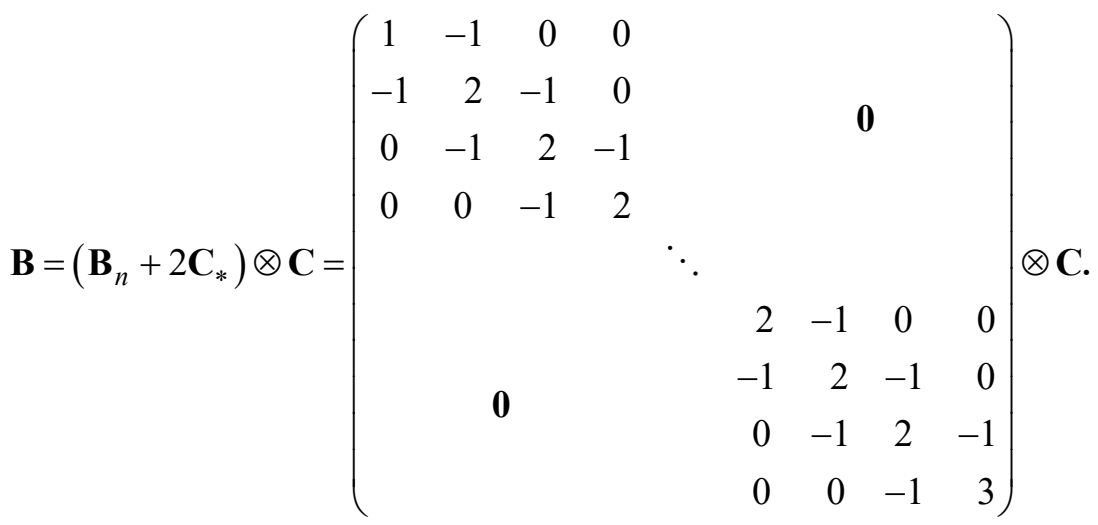

В таком случае уравнение (6.19) приобретает вид уравнения (6.20). Норма укороченного якобиана:

$$
\|\mathbf{A}(t)\|=\max \left(\left\|\mathbf{J}_{1}\left(\xi_{1}\right)\right\|,\left\|\mathbf{J}_{2}\left(\xi_{2}\right)\right\|, \ldots,\left\|\mathbf{J}_{n}\left(\xi_{n}\right)\right\|\right)=\left\|\mathbf{J}_{0}\left(\xi_{0}\right)\right\|=\mu .
$$

Здесь через $\mathbf{J}_{0}\left(\xi_{0}\right)$ переобозначен «парциальный» якобиан элементарного осциллятора с максимальной нормой. Известно, что минимальное собственное значение матрицы $\mathbf{B}_{n}+2 \mathbf{C}_{*}$ равно $\lambda_{\min }=4 \sin ^{2} \frac{\pi}{4 n}$ (см. Приложение II). Суммируя сказанное, сформулируем условия устойчивости тривиального решения (6.19) (структуры): кластерная структура в случае пары связанных КО устойчива 
при выполнении неравенств $\varepsilon>\mu / 4 \sin ^{2} \frac{\pi}{4 n}, \quad$ если $\mathbf{C}=\mathbf{I}_{m}$, и $\varepsilon>\varepsilon^{*}(\mu) / 4 \sin ^{2} \frac{\pi}{4 n}-$ в общем случае матрицы С. Данные условия носят достаточный характер.

Рассмотрим случай произвольного числа КО $O_{s}(n)$. Относительно векторов $\mathbf{Y}_{j}=\operatorname{col}\left(\mathbf{X}_{1 j}, \mathbf{X}_{2 j}, \ldots, \mathbf{X}_{n j}\right), j=1,2, \ldots, k, \quad$ имеем следующую систему уравнений:

$$
\begin{aligned}
& \dot{\mathbf{Y}}_{1}=\mathbf{G}\left(\mathbf{Y}_{1}\right)+\varepsilon\left(\mathbf{C}_{*} \otimes \mathbf{C}\right)\left(\mathbf{Y}_{2}-\mathbf{Y}_{1}\right), \\
& \dot{\mathbf{Y}}_{2}=\mathbf{G}\left(\mathbf{Y}_{2}\right)-\varepsilon\left(\mathbf{C}_{*} \otimes \mathbf{C}\right)\left(\mathbf{Y}_{2}-\mathbf{Y}_{1}\right)+\varepsilon\left(\mathbf{C}^{*} \otimes \mathbf{C}\right)\left(\mathbf{Y}_{3}-\mathbf{Y}_{2}\right), \\
& \dot{\mathbf{Y}}_{3}=\mathbf{G}\left(\mathbf{Y}_{3}\right)-\varepsilon\left(\mathbf{C}^{*} \otimes \mathbf{C}\right)\left(\mathbf{Y}_{3}-\mathbf{Y}_{2}\right)+\varepsilon\left(\mathbf{C}_{*} \otimes \mathbf{C}\right)\left(\mathbf{Y}_{4}-\mathbf{Y}_{3}\right), \\
& \vdots \\
& \dot{\mathbf{Y}}_{k}=\mathbf{G}\left(\mathbf{Y}_{k}\right)+\varepsilon\left(\mathbf{C}^{*} \otimes \mathbf{C}\right)\left(\mathbf{Y}_{k-1}-\mathbf{Y}_{k}\right),
\end{aligned}
$$

где $\mathbf{C}_{*}=\operatorname{diag}(0,0, \ldots, 0,1)$ и $\mathbf{C}^{*}=\operatorname{diag}(1,0, \ldots, 0,0)-$ матрицы синтеза.

Система (6.21) записана для нечетного $k$. В четном случае в последнем уравнении следует сделать замену $\mathbf{C}^{*} \rightarrow \mathbf{C}_{*}$. Исследуем достаточные условия устойчивости КС.

Линеаризованная система относительно векторов $\mathbf{U}_{j}=$ $=\mathbf{Y}_{j}-\mathbf{Y}_{j+1}$, имеет следующий вид:

$$
\begin{aligned}
& \dot{\mathbf{U}}_{1}=\mathbf{J}(\xi) \mathbf{U}_{1}-\varepsilon\left(2\left(\mathbf{C}_{*} \otimes \mathbf{C}\right) \mathbf{U}_{1}-\left(\mathbf{C}^{*} \otimes \mathbf{C}\right) \mathbf{U}_{2}\right), \\
& \dot{\mathbf{U}}_{2}=\mathbf{J}(\xi) \mathbf{U}_{2}-\varepsilon\left(-\left(\mathbf{C}_{*} \otimes \mathbf{C}\right) \mathbf{U}_{1}+2\left(\mathbf{C}^{*} \otimes \mathbf{C}\right) \mathbf{U}_{2}-\left(\mathbf{C}_{*} \otimes \mathbf{C}\right) \mathbf{U}_{3}\right), \\
& \dot{\mathbf{U}}_{3}=\mathbf{J}(\xi) \mathbf{U}_{3}-\varepsilon\left(-\left(\mathbf{C}^{*} \otimes \mathbf{C}\right) \mathbf{U}_{2}+2\left(\mathbf{C}_{*} \otimes \mathbf{C}\right) \mathbf{U}_{3}-\left(\mathbf{C}^{*} \otimes \mathbf{C}\right) \mathbf{U}_{4}\right),
\end{aligned}
$$

$$
\dot{\mathbf{U}}_{k-1}=\mathbf{J}(\xi) \mathbf{U}_{k-1}-\varepsilon\left(-\left(\mathbf{C}^{*} \otimes \mathbf{C}\right) \mathbf{U}_{k-2}+2\left(\mathbf{C}_{*} \otimes \mathbf{C}\right) \mathbf{U}_{k-1}\right) .
$$


Система (6.22), записанная одним уравнением:

$$
\begin{gathered}
\dot{\mathbf{U}}=\mathbf{I}_{k-1} \otimes\left(\operatorname{diag}\left(\mathbf{J}_{1}\left(\xi_{1}\right), \mathbf{J}_{2}\left(\xi_{2}\right), \ldots, \mathbf{J}_{n}\left(\xi_{n}\right)\right)-\varepsilon \mathbf{B}_{n} \otimes \mathbf{C}\right) \mathbf{U}- \\
-\varepsilon\left(\mathbf{D}_{n(k-1)} \otimes \mathbf{C}\right) \mathbf{U}
\end{gathered}
$$

где $\mathbf{U}=\left(\mathbf{U}_{1}, \mathbf{U}_{2} \ldots \mathbf{U}_{k-1}\right)^{T}$, а матрица $\mathbf{D}_{n(k-1)}$ :

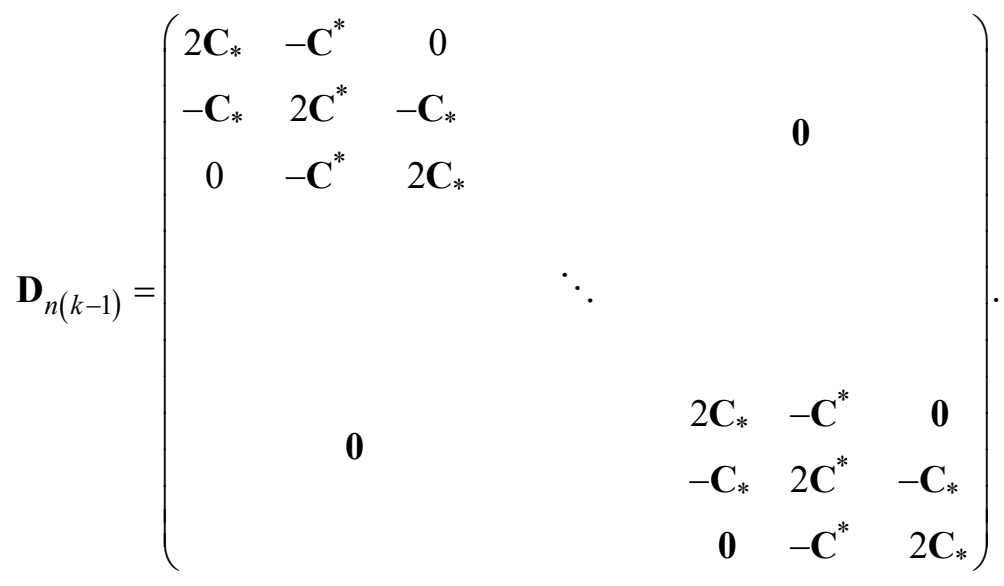

Подводя уравнение (6.23) под лемму 6.2, получаем: $\mathbf{A}(t)=$ $=\mathbf{I}_{n(k-1)} \otimes \operatorname{diag}\left(\mathbf{J}_{1}\left(\xi_{1}\right), \mathbf{J}_{2}\left(\xi_{2}\right), \ldots, \mathbf{J}_{n}\left(\xi_{n}\right)\right), \quad \mathbf{B}=\left(\mathbf{I}_{k-1} \otimes \mathbf{B}_{n}+\mathbf{D}_{n(k-1)}\right) \otimes \mathbf{C}$. Как видим, норма переменной матрицы остается прежней. Кроме того, минимальный корень матрицы $\mathbf{I}_{k-1} \otimes \mathbf{B}_{n}+\mathbf{D}_{n(k-1)} \quad$ есть $\lambda_{\min }=4 \sin ^{2} \frac{\pi}{2 n k}$. Все вместе дает условия устойчивости структуры, которые формулируются в прежнем виде.

3) Устойчивость кластерных структур на базе простых клеток $O_{a}(n)$.

Как известно, обобщенные осцилляторы типа $O_{a}(n)$ существуют парами, образуя простую клетку с определенной кластерной структурой [17] (рис. 6.52,a). 

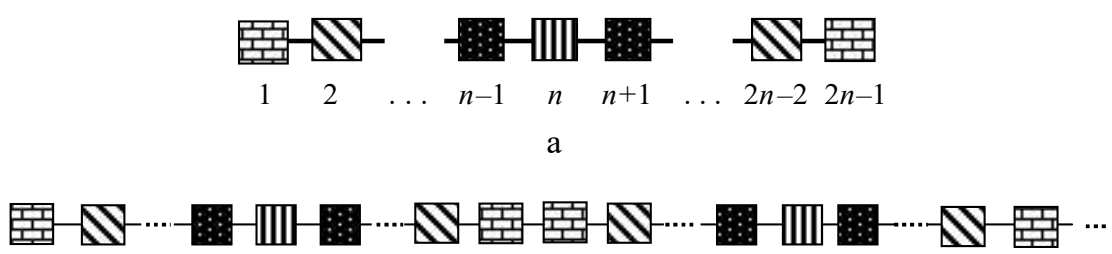

$\begin{array}{llllllllllll}1 & 2 & \ldots & n-1 & n & n+1 & \ldots & 2 n-2 & 2 n-1 & 2 n & 2 n+1 & \ldots\end{array}$

б

Рис. 6.52. Схема кластерной структуры простой клетки (а) и схема структуры при последовательном синтезе простых клеток (б).

Прежде всего, исследуем устойчивость структуры самой простой клетки, рассматривая ее уравнения как уравнения цепочки элементарных осцилляторов с числом $N=2 n-1$ :

$$
\begin{aligned}
& \dot{\mathbf{X}}_{i}=\mathbf{F}\left(\mathbf{X}_{i}\right)-\varepsilon \mathbf{C}\left(-\mathbf{X}_{i-1}+2 \mathbf{X}_{i}-\mathbf{X}_{i+1}\right), \\
& i=\overline{1, N}, \quad \mathbf{X}_{0}=\mathbf{X}_{1}, \quad \mathbf{X}_{N}=\mathbf{X}_{N+1} .
\end{aligned}
$$

В силу симметрии кластерной структуры линеаризацию (6.24) проводим по переменным $\mathbf{U}_{i}=\mathbf{X}_{i}-\mathbf{X}_{N-i+1}, i=\overline{1, n-1}$. Линеаризованная система имеет вид

$$
\begin{aligned}
& \dot{\mathbf{U}}_{i}=\mathbf{J}_{i}\left(\xi_{i}\right) \mathbf{U}_{i}-\varepsilon \mathbf{C}\left(-\mathbf{U}_{i-1}+2 \mathbf{U}_{i}-\mathbf{U}_{i+1}\right), \\
& i=\overline{1, n-1}, \quad \mathbf{U}_{0}=\mathbf{U}_{1}, \quad \mathbf{U}_{n}=0 .
\end{aligned}
$$

Система (6.25) в форме одного уравнения

$$
\dot{\mathbf{U}}=\left(\mathbf{J}^{*}(\xi)-\varepsilon \mathbf{D}_{p} \otimes \mathbf{C}\right) \mathbf{U},
$$

где $\mathbf{J}^{*}(\xi)=\operatorname{diag}\left(\mathbf{J}_{1}\left(\xi_{1}\right), \mathbf{J}_{2}\left(\xi_{2}\right), \ldots, \mathbf{J}_{n-1}\left(\xi_{n-1}\right)\right)$ - укороченный якобиан КО $O_{a}(n),\left(\xi_{1}, \xi_{2}, \ldots, \xi_{n}\right) \in A_{a}(n)$, а матрица $\mathbf{D}_{p}$ имеет вид 


$$
\mathbf{D}_{p}=\left\|\begin{array}{ccccccc}
1 & -1 & 0 & \cdots & \cdots & 0 & 0 \\
-1 & 2 & -1 & \ddots & \cdots & 0 & 0 \\
0 & -1 & 2 & \ddots & \ddots & 0 & 0 \\
\vdots & \ddots & \ddots & \ddots & \ddots & \ddots & \vdots \\
\vdots & \vdots & \ddots & \ddots & 2 & -1 & 0 \\
0 & 0 & 0 & \ddots & -1 & 2 & -1 \\
0 & 0 & 0 & \cdots & 0 & -1 & 2
\end{array}\right\| .
$$

Как видим, уравнение (6.26) полностью подпадает под лемму 6.2. Норма переменной матрицы остается прежней, а минимальный корень матрицы $\mathbf{D}_{p}$ имеет выражение $\lambda_{\min }=4 \sin ^{2} \frac{\pi}{2(2 n-1)}$. Таким образом, условия устойчивости кластерной структуры ПК имеют уже знакомый вид: $\varepsilon>\mu / \lambda_{\min }$, если $\mathbf{C}=\mathbf{I}$, и $\varepsilon>\varepsilon^{*}(\mu) / \lambda_{\min }$ в случае матрицы $\mathbf{C}$ общего вида.

Рассматриваем структуры на базе простых клеток при параллельном и последовательном синтезе. Будем предполагать, что условия устойчивости структуры самой ПК выполнены, и рассматривать устойчивость синхронизации совокупностей этих внутренне структурированных субъектов. Очевидно, что при параллельном синтезе (рис. 6.53) мы получим те же самые условия устойчивости, что и в случае КО $O_{s}(n)$, для адаптации которых следует лишь осуществить замену $\lambda_{s}(n) \rightarrow \lambda_{a}(n)$.

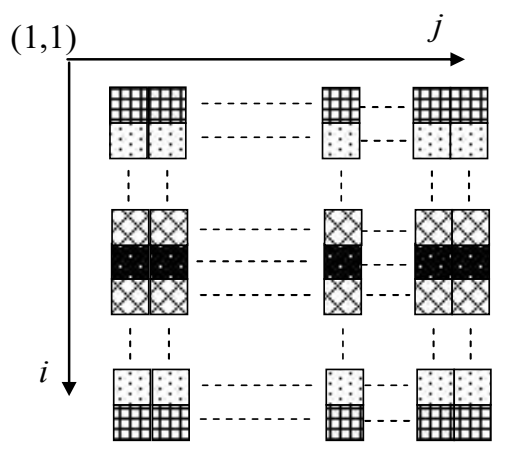

Рис. 6.53. Кластерная структура при параллельном синтезе. 
В случае последовательного синтеза (см. рис. 6.52,б) ситуация также упрощается за счет симметрии внутренней структуры ПК: матрицей последовательного синтеза может всегда являться $\mathbf{C}_{*}=\operatorname{diag}(0,0, \ldots, 0,1) \quad$ или $\mathbf{C}^{*}=\operatorname{diag}(1,0, \ldots, 0,0) \quad$ с размерностью $2 n-1$; какая из них - не имеет значения. То есть можно воспользоваться системой (6.22) после замены в ней $\mathbf{C}^{*} \rightarrow \mathbf{C}_{*}$. В этом случае матрица $\mathbf{D}_{(2 n-1)(k-1)}$ (замена $\left.n \rightarrow 2 n-1\right) \quad$ принимает $\quad$ вид $\mathbf{D}_{(2 n-1)(k-1)}=\mathbf{D}_{k-1} \otimes \mathbf{C}_{*}$, где

$$
\mathbf{D}_{k-1}=\left\|\begin{array}{|ccccccc}
2 & -1 & 0 & \cdots & \cdots & 0 & 0 \\
-1 & 2 & -1 & \ddots & \cdots & 0 & 0 \\
0 & -1 & 2 & \ddots & \ddots & 0 & 0 \\
\vdots & \ddots & \ddots & \ddots & \ddots & \ddots & \vdots \\
\vdots & \vdots & \ddots & \ddots & 2 & -1 & 0 \\
0 & 0 & 0 & \ddots & -1 & 2 & -1 \\
0 & 0 & 0 & \cdots & 0 & -1 & 2
\end{array}\right\| .
$$

С учетом сказанного уравнение (6.23) перепишется следующим образом:

$$
\dot{\mathbf{U}}=\mathbf{I}_{k-1} \otimes \mathbf{J}(\xi) \mathbf{U}-\varepsilon\left(\mathbf{D}_{k-1} \otimes \mathbf{C}_{*} \otimes \mathbf{C}\right) \mathbf{U},
$$

где $\mathbf{J}(\xi)=\operatorname{diag}\left(\mathbf{J}_{1}\left(\xi_{1}\right), \mathbf{J}_{2}\left(\xi_{2}\right), \ldots, \mathbf{J}_{2 n-1}\left(\xi_{1}\right)\right)-\varepsilon \mathbf{B}_{2 n-1} \otimes \mathbf{C}-$ якобиан ПК.

Как видим, уравнение (6.27) подпадает под лемму 5.1, и, следовательно, задача устойчивости (6.27) сводится к соответствующей задаче для уравнения вида

$$
\dot{\mathbf{U}}=\mathbf{J}(\xi) \mathbf{U}-\varepsilon \lambda_{\min } \mathbf{C}_{*} \otimes \mathbf{C U},
$$

где $\lambda_{\min }=4 \sin ^{2} \frac{\pi}{2 k}$ - минимальный корень матрицы $\mathbf{D}_{k-1}$.

Уравнение (6.28) не является конечным пунктом решения задачи, поскольку для него имеется та же проблема, что и для уравнения (6.19). Поэтому продолжаем преобразования (6.28) под лемму 6.2, проделывая те же процедуры, что и с уравнением (6.19). 
В результате получаем норму для переменной матрицы как наибольшую из норм парциальных якобианов осцилляторов ПК, а также минимальное собственное значение постоянной матрицы минимальный корень $\Lambda_{\min }$ матрицы $\mathbf{B}_{2 n-1}+\lambda_{\min } \mathbf{C}_{*}$ :

$$
\mathbf{B}_{2 n-1}+\lambda_{\min } \mathbf{C}_{*}=\left\|\begin{array}{crrrrrr||}
1 & -1 & 0 & \cdots & \cdots & 0 & 0 \\
-1 & 2 & -1 & \ddots & \cdots & 0 & 0 \\
0 & -1 & 2 & \ddots & \ddots & 0 & 0 \\
\vdots & \ddots & \ddots & \ddots & \ddots & \ddots & \vdots \\
\vdots & \vdots & \ddots & \ddots & 2 & -1 & 0 \\
0 & 0 & 0 & \ddots & -1 & 2 & -1 \\
0 & 0 & 0 & \cdots & 0 & -1 & a
\end{array}\right\|, \quad a=1+4 \sin ^{2} \frac{\pi}{2 k}
$$

В частности, если $k=2$, то $\Lambda_{\min }=4 \sin ^{2} \frac{\pi}{4(2 n-1)} ;$ если $k=3$, то $\Lambda_{\min }=4 \sin ^{2} \frac{\pi}{2(4 n-1)}$. Кластерная структура (см. рис. 6.54) устойчива при $\varepsilon>\mu / \Lambda_{\min }$, если $\mathbf{C}=\mathbf{I}$, и $\varepsilon>\varepsilon^{*}(\mu) / \Lambda_{\min }-$ в случае матрицы $\mathbf{C}$ общего вида.

Еще раз подчеркнем, что свойства кластерных аттракторов в сильной степени зависят от параметра решетки $\varepsilon$ : если какой-то из них является хаотическим при относительно небольшом $\varepsilon$, то с увеличением этого параметра аттрактор достаточно быстро становится регулярным, а далее исчезает вовсе. То есть полученные условия устойчивости для параметра $\varepsilon$ имеют ограничения сверху, связанные с типом конкретного аттрактора и его существованием. В частности, это влечет за собой ограничения на число кластеров в устойчивых структурах. В типичном случае кластерные структуры с хаотической внутренней динамикой, которые существуют при «умеренных» $\varepsilon$, являются мультистабильными - устойчивыми по одним направлениям в фазовом пространстве и неустойчивыми по другим направления. Это приводит к тому, что в численном или натурном эксперименте вместо одной кластерной структуры может наблюдаться калейдоскоп сменяющих во времени друг друга кластерных структур. 
Приложение I

\section{АЛГОРИТМЫ ПРЕОБРАЗОВАНИЯ СИСТЕМ СВЯЗАННЫХ РОТАТОРОВ К СТАНДАРТНОЙ ФОРМЕ}

\section{П.1. Системы связанных ротаторов без дополнительных нагрузок}

Рассмотрим случай, когда каждый из ротаторов не нагружен какими-либо дополнительными апериодическими или колебательными нагрузками, а система (П1) имеет вид

$$
\begin{aligned}
& I \ddot{\varphi}_{k}+\delta_{k}\left(1+F_{1 k}\left(\varphi_{k}\right)\right) \dot{\varphi}_{k}+F_{2 k}\left(\varphi_{k}\right)= \\
& =\gamma_{k}+\sum_{j=1}^{n} f_{k j}\left(\varphi_{j}\right)+\sum_{j=1}^{n} \beta_{k j} \dot{\varphi}_{j}+f_{k}(\psi)+\mu F_{k}^{*}(\varphi), \\
& \dot{\psi}=\omega_{0},
\end{aligned}
$$

где $\left\langle f_{k j}\left(\varphi_{j}\right)\right\rangle_{\varphi_{j}}=0,\left\langle f_{k}(\psi)\right\rangle_{\psi}=0, \mu=I^{-1}$.

Другими словами, рассмотрим систему ротаторов, связанных по угловым скоростям, угловым ускорениям, вследствие чего возникают связи «по периодическим функциям», а также с «прочими связями», которые учтены членами $\mu F_{k}^{*}(\varphi)$.

Решаем задачу о виде преобразования $(\varphi, \dot{\varphi}) \rightarrow(\varphi, \xi)$, приводящего (П.1.1) к системе в стандартной форме с быстровращающимися фазами

$$
\begin{aligned}
& \dot{\xi}_{k}=\mu \Xi_{k}(\varphi, \psi, \xi), \\
& \dot{\varphi}_{k}=\omega_{k}+\mu \Phi_{k}\left(\varphi, \psi, \xi_{k}\right), \\
& \dot{\psi}=\omega_{0},
\end{aligned}
$$

где $\omega_{k}, \Xi_{k}(\varphi, \psi, \xi), \Phi_{k}\left(\varphi, \psi, \xi_{k}\right)$ - неизвестные параметры и функции преобразования, подлежащие определению. 
Требованием для функций $\Xi_{k}(\varphi, \psi, \xi)$ и $\Phi_{k}\left(\varphi, \psi, \xi_{k}\right)$ является их ограниченность по всем фазовым переменным (отсутствие в этих функциях «вековых» членов по $\varphi$ ), что фактически соответствует их периодичности по этим переменным.

Подстановка второго уравнения (П.1.2) в (П.1.1) приводит к уравнению, определяющему искомые параметры и функции преобразования:

$$
\begin{gathered}
\sum_{j=1}^{n} \frac{\partial \Phi_{k}}{\partial \varphi_{j}}\left(\omega_{j}+\mu \Phi_{j}\right)+\frac{\partial \Phi_{k}}{\partial \psi} \omega_{0}+\dot{\xi}_{k}+\delta_{k}\left(1+F_{1 k}\right)\left(\omega_{k}+\mu \Phi_{k}\right)+F_{2 k}= \\
=\gamma_{k}+\sum_{j=1}^{n} f_{k j}+\sum_{j=1}^{n} \beta_{k j}\left(\omega_{j}+\mu \Phi_{j}\right)+f_{k}(\psi)+\mu F_{k}^{*}(\varphi) .
\end{gathered}
$$

Из условия ограниченности получаем уравнения для нормальных частот ротаторов:

$$
\delta_{k} \omega_{k}-\sum_{j=1}^{n} \beta_{k j} \omega_{j}=\gamma_{k}, k=\overline{1, n} .
$$

Эти уравнения - результат «изъятия» из уравнения (П.1.3) всех констант.

Принимая во внимание форму первого уравнения (П.1.2) $(\dot{\xi} \sim \mu)$, получаем уравнения, определяющие функции $\Phi_{k}$ (изъятие из уравнения членов, непропорциональных $\mu$ ):

$$
\begin{gathered}
\sum_{j=1}^{n} \frac{\partial \Phi_{k}}{\partial \varphi_{j}} \omega_{j}+\frac{\partial \Phi_{k}}{\partial \psi} \omega_{0}+\delta_{k} \omega_{k} F_{1 k}\left(\varphi_{k}\right)+F_{2 k}\left(\varphi_{k}\right)= \\
=\sum_{j=1}^{n} f_{k j}\left(\varphi_{j}\right)+f_{k}(\psi) .
\end{gathered}
$$

Линейную систему (П.1.4) решаем без труда. Уравнение (П.1.5) также простое. Нужное нам решение имеет вид

$$
\begin{gathered}
\Phi_{k}=\sum_{j=1}^{n} \frac{1}{\omega_{j}} \int f_{k j}\left(\varphi_{j}\right) d \varphi_{j}+\frac{1}{\omega_{0}} \int f_{k}(\psi) d \psi-\delta_{k} \int F_{1 k}\left(\varphi_{k}\right) d \varphi_{k}- \\
-\frac{1}{\omega_{k}} \int F_{2 k}\left(\varphi_{k}\right) d \varphi_{k}+\xi_{k} .
\end{gathered}
$$


Эти функции ограничены по фазовым переменным (как следствие «изъятия» констант).

При этом для функций $\Xi_{k}$ получаем выражения

$$
\Xi_{k}=\sum_{j=1}^{n}\left(\beta_{k j}-\frac{\partial \Phi_{k}}{\partial \varphi_{j}}\right) \Phi_{j}-\delta_{k} \Phi_{k}\left(1+F_{1 k}\right)+F_{k}^{*}
$$

Как уже говорилось, в квазилинейных системах «сильными» являются только гармонические резонансы, соответствующие целому отношению частот. В данном случае под сильным резонансом мы подразумеваем резонанс, проявляющийся в первом приближении усредненной системы. Субгармонические резонансы в этом смысле являются слабыми. Из числа гармонических резонансов, как правило, наиболее значимым является первый - главный - резонанс. В рассматриваемых системах он соответствует простой взаимной синхронизации колебаний, а все другие - кратной. То же самое относится к принудительной синхронизации автоколебательной системы внешним воздействием. Для исследования гармонических резонансов в системе (П.1.2) вводим фазовые и частотные расстройки вида $q_{k} \varphi_{k}-p_{k} \psi=\eta_{k}, \quad q_{k} \omega_{k}-p_{k} \omega_{0}=\mu \Delta_{k}, \quad$ где $p_{k} / q_{k}=n_{k}$ - целые числа ( $n_{k}=1$ - простая синхронизация). В итоге получается система с одной быстрой фазой $\psi$ вида

$$
\begin{aligned}
& \dot{\xi}_{k}=\mu \Xi_{k}(\eta, \psi, \xi), \\
& \dot{\eta}_{k}=\mu\left(\Delta_{k}+q_{k} \Phi_{k}\left(\eta, \psi, \xi_{k}\right)\right), \\
& \dot{\psi}=\omega_{0} .
\end{aligned}
$$

В результате процедуры усреднения получаем:

$$
\Xi_{k}^{*}(\eta, \mathbf{x})=\frac{1}{2 \pi} \int_{0}^{2 \pi} \Xi_{k}(\eta, \mathbf{x}, \psi) \partial \psi, \frac{1}{2 \pi} \int_{0}^{2 \pi}\left(\Delta_{k}+q_{k} \Phi_{k}\right) \partial \psi=\Delta_{k}+q_{k} \xi_{k}
$$

и соответствующую усредненную систему

$$
\begin{aligned}
& \dot{\xi}_{k}=\Xi_{k}^{*}(\eta, \xi), \\
& \dot{\eta}_{k}=\Delta_{k}+q_{k} \xi_{k} .
\end{aligned}
$$


В системе (П.1.7) за усредненными переменными сохранены старые обозначения и преобразовано время $\mu \tau=\tau_{n}$. Как результат исследования системы (П.1.7), получаем, в частности, ХВ ротаторов в резонансных зонах параметров:

$$
\left\langle\dot{\varphi}_{k}\right\rangle_{\tau}=\Omega_{k}=n_{k} \omega_{0}+\frac{\mu}{q_{k}}\left\langle\dot{\eta}_{k}^{*}\right\rangle_{\tau}
$$

При интерпретации динамических свойств усредненной системы (П.1.7) на исходную систему (П1) учитываем, что если $G$ - предельное множество траекторий усредненной системы, то $G \times S^{1}$ предельное множество траекторий исходной системы с оговорками, сделанными нами по тексту. Учитываем также связь траекторий усредненной системы с траекториями точечного отображения Пуанкаре, построенного для исходной системы.

Замечание 1. Выбор быстрой, «усредняющей» фазы в системе (П.1.2) определяется соображениями удобства. Если система неавтономна, то таковой является фаза внешнего воздействия $\psi$. Если система (П.1.1) и, соответственно, (П.1.2) автономны, то в качестве «усредняющей» фазы может быть выбрана любая. Если в таком качестве выбирается $\varphi_{1}=\varphi$, то для исследования синхронизации вводятся фазовые и частотные расстройки вида $q_{k} \varphi_{k}-p_{k} \varphi=\eta_{k}, q_{k} \omega_{k}-p_{k} \omega_{1}=\mu \Delta_{k}$, где $p, q$ - целые числа.

Замечание 2. Если система (П.1.1) неавтономна, то при выполнении преобразований технически удобнее задавать форму уравнений для фаз ротаторов в виде $\dot{\varphi}_{k}=n_{k} \omega_{0}+\mu \Phi_{k}\left(\varphi, \psi, \xi_{k}\right)$. Если система автономна, то $\dot{\varphi}_{k}=n_{k} \omega+\mu \Phi_{k}\left(\varphi, \xi_{k}\right)$, где $\omega-$ нормальная частота «усредняющего» ротатора, $n_{k}$ - целые числа. При этом частотные расстройки переходят в уравнение для переменной $\xi$. Заметим, что выбор частоты не влияет на конечный результат, но сокращает процедуру преобразований усредненных систем. 


\section{П.2. Системы связанных ротаторов с апериодическими нагрузками}

Сначала рассмотрим один ротатор с апериодической нагрузкой, уравнения которой имеют произвольную размерность. Предположим, что матрица А имеет действительные корни и, не теряя общности, считаем ее диагональной. Также предположим, что нагрузка обладает малой диссипацией, т. е. $\mathbf{A}=\mu \mathbf{B}$, где $\mathbf{B}-$ диагональная невырожденная матрица. При сделанных предположениях рассматриваем динамическую систему вида

$$
\begin{aligned}
& I \ddot{\varphi}+\delta\left(1+F_{1}(\varphi)\right) \dot{\varphi}+F_{2}(\varphi)=\gamma+\mathbf{a}^{T} \mathbf{z}+f(\psi)+\mu F^{*}(\varphi, \mathbf{z}), \\
& \dot{\mathbf{z}}=\mu\left(\mathbf{B z}+\mathbf{b} \dot{\varphi}+\mathbf{c} f_{1}(\varphi)+\mathbf{d} f_{2}(\psi)\right),
\end{aligned}
$$

где $\mathbf{a}, \mathbf{b}, \mathbf{c}, \mathbf{d}-$ постоянные векторы.

Легко видеть, что «порождающим» решением второго уравнения (решение при $\mu=0$ ) является $\mathbf{z}=$ const. Это значит, что если уравнение ротатора преобразовывать к стандартной форме исходя непосредственно из (П.2.1), то переменная $\mathbf{z}$ будет включаться в «порождающую» частоту ротатора. Это обстоятельство в немалой степени усложнило бы всю дальнейшую процедуру усреднения. Идеальным является случай, когда «порождающая» частота не зависит от «медленных» переменных, т. е. является параметром. По этой причине выполним следующее преобразование уравнения нагрузки

$$
\mathbf{z}=\boldsymbol{\beta}+\mu \Psi(\varphi, \psi)+\mu \mathbf{y},
$$

где постоянный вектор $\beta$ и вектор-функция $\Psi(\varphi, \psi)$ подлежат определению. Подставляя уравнение (П.2.2) во второе уравнение (П.2.1) и учитывая, что уравнение для фазы $\varphi$ имеет вид $\dot{\varphi}=\omega_{0}+\mu \Phi$, получаем

$$
\begin{gathered}
\frac{\partial \Psi}{\partial \varphi}\left(\omega_{0}+\mu \Phi\right)+\frac{\partial \Psi}{\partial \psi} \omega_{0}+\dot{\mathbf{y}}= \\
=\mu \mathbf{B}(\Psi+\mathbf{y})+\mathbf{B} \beta+\mathbf{b} \omega_{0}+\mu \mathbf{b} \Phi+\mathbf{c} f_{1}(\varphi)+\mathbf{d} f_{2}(\psi) .
\end{gathered}
$$


Уравнения, определяющие $\boldsymbol{\beta}$ и $\mathbf{Y}(\varphi, \psi)$, получаем из следующих простых соображений: во-первых, уравнение для переменной у должно иметь стандартную форму, во-вторых, функция $\mathbf{Y}(\varphi, \psi)$ должна быть ограниченной. При этих условиях искомые уравнения имеют вид

$$
\begin{aligned}
& \frac{\partial \Psi}{\partial \varphi} \omega_{0}+\frac{\partial \Psi}{\partial \psi} \omega_{0}=\mathbf{c} f_{1}(\varphi)+\mathbf{d} f_{2}(\psi), \\
& \mathbf{B} \beta+\mathbf{b} \omega_{0}=0 .
\end{aligned}
$$

Решение уравнений (П.2.4) не составляет труда:

$$
\begin{aligned}
& \Psi=\frac{\mathbf{c}}{\omega_{0}} \int f_{1}(\varphi) d \varphi+\frac{\mathbf{d}}{\omega_{0}} \int f_{2}(\psi) d \psi, \\
& \boldsymbol{\beta}=-\mathbf{B}^{-1} \mathbf{b} \omega_{0} .
\end{aligned}
$$

При найденных решениях уравнения (П.2.1) приобретают вид

$$
\begin{aligned}
& I \ddot{\varphi}+\delta\left(1+F_{1}(\varphi)\right) \dot{\varphi}+F_{2}(\varphi)=\gamma+\mathbf{a}^{T} \boldsymbol{\beta}+f(\psi)+\mu \mathbf{a}^{T}(\Psi+\mathbf{y})+\mu F^{*}(\varphi, \mathbf{z}), \\
& \dot{\mathbf{y}}=\mu\left(\mathbf{B y}+\mathbf{B} \Psi-\frac{\partial \Psi}{\partial \varphi} \Phi+\mathbf{b} \Phi\right) .
\end{aligned}
$$

Далее преобразуем уравнение ротатора. Подставляя в первое уравнение (П.2.5) уравнение для фазы $\dot{\varphi}=\omega_{0}+\mu \Phi(\varphi, \psi, \xi)$, получаем

$$
\begin{aligned}
& \frac{\partial \Phi}{\partial \varphi}\left(\omega_{0}+\mu \Phi\right)+\frac{\partial \Phi}{\partial \psi} \omega_{0}+\frac{\partial \Phi}{\partial \xi} \dot{\xi}+\delta\left(1+F_{1}(\varphi)\right)\left(\omega_{0}+\mu \Phi\right)+F_{2}(\varphi)= \\
& =\gamma+\mathbf{a}^{T} \beta+f(\psi)+\mu \mathbf{a}^{T}(\Psi+\mathbf{y})+\mu F^{*}(\varphi, \mathbf{z}) .
\end{aligned}
$$

Уравнение для функции $\Phi(\varphi, \psi, \xi)$ получаем из тех же условий, что и в (П1):

$$
\frac{\partial \Phi}{\partial \varphi} \omega_{0}+\frac{\partial \Phi}{\partial \psi} \omega_{0}+\delta \omega_{0} F_{1}(\varphi)+F_{2}(\varphi)=f(\psi) .
$$

Его решение $\Phi=-\delta \int F_{1}(\varphi) d \varphi-\frac{1}{\omega_{0}} \int F_{2}(\varphi) d \varphi+\frac{1}{\omega_{0}} \int f(\psi) d \psi+\xi$. 
Соотношение $\gamma+\mathbf{a}^{T} \beta-\delta \omega_{0}=\mu \Delta$ определяет резонансную зону параметров.

В итоге получаем систему уравнений в стандартной форме, эквивалентную системе (П.2.1):

$$
\begin{aligned}
& \dot{\xi}=\mu \Xi, \\
& \dot{\mathbf{y}}=\mu \mathbf{Y}, \\
& \dot{\eta}=\mu \Phi, \\
& \dot{\varphi}=\omega_{0}+\mu \Phi,
\end{aligned}
$$

где $\Xi=\Delta-\delta\left(1+F_{1}(\varphi)\right) \Phi-\frac{\partial \Phi}{\partial \varphi} \Phi+\mathbf{a}^{T}(\Psi+\mathbf{y})+F^{*}(\varphi, \mathbf{z}), \quad \mathbf{Y}=\mathbf{B y}+$ $+\mathbf{B} \Psi-\frac{\partial \Psi}{\partial \varphi} \Phi+\mathbf{b} \Phi, \eta=\varphi-\psi-$ фазовая расстройка.

Замечание 3. Мы рассмотрели случай неавтономной системы. Здесь порождающая частота ротатора была определена и являлась частотой внешнего воздействия $\omega_{0}$. В автономном случае порождающая частота ротатора заранее не определена. Она находится из резонансного соотношения: полагая $\Delta=0, \quad$ получаем $\omega_{0}=\frac{\gamma}{\left(\mathbf{a}^{T} \mathbf{B}^{-1} \mathbf{b}+\delta\right)}-$ порождающую частоту ротатора.

Рассмотрим систему произвольного числа связанных ротаторов с апериодическими нагрузками. Допустим, что уравнения всех апериодических нагрузок преобразованы по образцу, представленному выше, и система связанных ротаторов имеет вид

$$
\begin{aligned}
& I \ddot{\varphi}_{k}+\delta\left(1+F_{1 k}\left(\varphi_{k}\right)\right) \dot{\varphi}_{k}+F_{2 k}\left(\varphi_{k}\right)=\gamma_{k}+\mathbf{a}_{k}^{T} \beta_{\kappa}+f_{k}(\psi)+\sum_{j=1}^{n} f_{k j}\left(\varphi_{j}\right)+ \\
& +\sum_{j=1}^{n} \beta_{k j} \dot{\varphi}_{j}+\mu \mathbf{a}_{k}^{T}\left(\Psi_{k}+\mathbf{y}_{k}\right)+\mu F_{k}^{*}(\varphi, \mathbf{z}), \\
& \dot{\mathbf{y}}_{k}=\mu \mathbf{Y}_{k}, \\
& \dot{\psi}=\omega_{0} .
\end{aligned}
$$


Преобразование уравнений ротаторов в системе (П.2.8) не будет отличаться от соответствующих преобразований в системе (П.1.1). Более того, поскольку воздействие нагрузок на ротаторы в системе (П.2.8) «слабое», то функции $\Phi_{k}$ не будут зависеть от векторов $y_{k}$. Они будут иметь те же самые выражения, что и в (П1).

После выполнения преобразований и определения необходимых функций получаем систему в стандартной форме, эквивалентную системе (П.2.8):

$$
\begin{aligned}
& \dot{\xi}_{k}=\mu \Xi_{k}\left(\varphi, \psi, \xi, \mathbf{y}_{k}\right), \\
& \dot{\mathbf{y}}_{k}=\mu \mathbf{Y}_{k}\left(\varphi, \psi, \xi, \mathbf{y}_{k}\right), \\
& \dot{\varphi}_{k}=\omega_{k}+\mu \Phi_{k}\left(\varphi, \psi, \xi_{k}\right), \\
& \dot{\psi}=\omega_{0} .
\end{aligned}
$$

Уравнения и, соответственно, выражения для функций $\Phi_{k}$ такие же, как и в (П1):

$$
\begin{gathered}
\Phi_{k}=\sum_{j=1}^{n} \frac{1}{\omega_{j}} \int f_{k j}\left(\varphi_{j}\right) d \varphi_{j}+\frac{1}{\omega_{0}} \int f_{k}(\psi) d \psi-\delta_{k} \int F_{1 k}\left(\varphi_{k}\right) d \varphi_{k}- \\
-\frac{1}{\omega_{k}} \int F_{2 k}\left(\varphi_{k}\right) d \varphi_{k}+\xi_{k} .
\end{gathered}
$$

Выражения для функций $\Xi_{k}$ и $Y_{k}$ :

$$
\begin{gathered}
\Xi_{k}=\sum_{j=1}^{n}\left(\beta_{k j}-\frac{\partial \Phi_{k}}{\partial \varphi_{j}}\right) \Phi_{j}-\delta_{k} \Phi_{k}\left(1+F_{1 k}\right)+F_{k}^{*}+\mathbf{a}_{k}^{T}\left(\Psi_{k}+\mathbf{y}_{k}\right), \\
\mathbf{Y}_{k}=\mathbf{B}_{k} \mathbf{y}_{k}+\mathbf{B}_{k} \Psi_{k}-\frac{\partial \Psi_{k}}{\partial \varphi_{k}} \Phi_{k}+\mathbf{b}_{k} \Phi_{k}
\end{gathered}
$$

соответственно, уравнения для порождающих частот ротаторов

$$
\begin{aligned}
& \left(\delta_{k}+\mathbf{a}_{k}^{T} \mathbf{B}_{k}^{-1} \mathbf{b}_{k}\right) \omega_{k}-\sum_{j=1}^{n} \beta_{k j} \omega_{j}=\gamma_{k}, \\
& k=\overline{1, n} .
\end{aligned}
$$


Далее, в соответствии с замечаниями 1, 2, система (П.2.9) сводится к системе в стандартной форме с одной быстровращающейся фазой и осуществляется процедура усреднения.

\section{П.3. Системы связанных ротаторов с колебательными нагрузками}

Сначала рассмотрим случай одного ротатора, взаимодействующего с многочастотной колебательной нагрузкой.

Как уже говорилось, квазилинейный ротатор эффективно взаимодействует с колебательной нагрузкой только в резонансном случае и при условии, что соответствующий осциллятор будет обладать высокой добротностью (малым затуханием). Предположим, что все диссипативные члены осцилляторов системы отнесены к функции $\mathbf{Z}(\mathbf{z}, \varphi, \dot{\varphi}, \psi)$ системы (П1). Будем также считать, что все частоты осцилляторов находятся в отношении к наименьшей из частот (или к частоте внешнего воздействия), близком к целочисленному, т. е. все частоты представляются в виде $\omega_{j}^{2}=n_{j}^{2} \omega_{0}^{2}+\mu v_{j}, n_{j}$ - целые числа. Полагаем, что все члены уравнения, связанные с расстройками частот $\left(\mu \nu_{j}\right)$, отнесены к функции $\mathbf{Z}(\mathbf{z}, \varphi, \dot{\varphi}, \psi)$. Кроме того, полагаем, что векторное уравнение колебательной системы преобразовано к состоянию равновесия в начале координат. Названные условия легко удовлетворяются элементарными преобразованиями уравнений колебательной системы, и мы считаем эти преобразования выполненными.

С учетом сказанного рассмотрим динамическую систему вида

$$
\begin{aligned}
& I \ddot{\varphi}+\delta\left(1+F_{1}(\varphi)\right) \dot{\varphi}+F_{2}(\varphi)=\gamma+\mathbf{a}^{T} \mathbf{z}+f(\psi)+\mu F^{*}(\varphi, \mathbf{z}), \\
& \dot{z}=\mathbf{A z}+\mu \mathbf{Z}(\mathbf{z}, \varphi), \\
& \dot{\psi}=\omega_{0},
\end{aligned}
$$


где $\mathbf{a}$ - постоянный вектор, матрица А имеет мнимые собственные значения и жорданову форму с клетками вида $\mathbf{A}_{j}=\left(\begin{array}{cc}0 & 1 \\ -n_{j}^{2} \omega_{0}^{2} & 0\end{array}\right)$, $j=\overline{1, m}, \operatorname{dim}(\mathbf{z}, \mathbf{Z})=2 m$.

Нетрудно видеть, что порождающее векторное уравнение колебательной системы (уравнение при $\mu=0$ ) является системой независимых гармонических осцилляторов. Переобозначим переменные: $z_{2 j-1}=x_{j}, Z_{2 j-1}=X_{j}, z_{2 j}=y_{j}, Z_{2 j}=Y_{j}$.

В соответствии с замечанием 2 определим форму уравнения для фазы ротатора в виде $\dot{\varphi}=\omega_{0}+\mu \Phi$ и преобразуем все квазилинейные уравнения осцилляторов при помощи модифицированной замены Ван дер Поля по единому образцу: в каждой системе вида

$$
\dot{x}=y+\mu X, \quad \dot{y}=-n^{2} \omega_{0}^{2} x+\mu Y
$$

сделаем замену

$$
x=\theta \sin n \varphi+\eta \cos n \varphi, \quad y=(\theta \cos n \varphi-\eta \sin n \varphi) n \omega_{0},
$$

в результате чего получаем систему осцилляторов в виде

$$
\dot{\theta}=\mu \Theta, \dot{\eta}=\mu T,
$$

где

$$
\begin{aligned}
& \Theta=n \eta \Phi+X \sin n \varphi+\frac{1}{n \omega_{0}} Y \cos n \varphi, \\
& T=-n \theta \Phi+X \cos n \varphi-\frac{1}{n \omega_{0}} Y \sin n \varphi .
\end{aligned}
$$

В новых переменных уравнения колебательной системы имеют стандартную форму относительно $\theta=\left(\theta_{1}, \ldots, \theta_{m}\right)^{T}$, и $\eta=\left(\eta_{1}, \ldots, \eta_{m}\right)^{T}$, а сама система (П.3.1) приобретает вид

$$
\begin{aligned}
& I \ddot{\varphi}+\delta\left(1+F_{1}(\varphi)\right) \dot{\varphi}+F_{2}(\varphi)=\gamma+b^{T} x+c^{T} y+f(\psi)+\mu F^{*}(\varphi, z), \\
& \dot{\theta}=\mu \Theta, \\
& \dot{\eta}=\mu \mathbf{T}, \\
& \dot{\psi}=\omega_{0},
\end{aligned}
$$


где $\quad \mathbf{b}^{T} \mathbf{x}=\sum_{i=1}^{m} b_{i}\left(\theta_{i} \sin n_{i} \varphi+\eta_{i} \cos n_{i} \varphi\right), \quad \mathbf{c}^{T} \mathbf{y}=\sum_{i=1}^{m} c_{i}\left(\theta_{i} \cos n_{i} \varphi-\right.$ $\left.-\eta_{i} \sin n_{i} \varphi\right) n_{i} \omega_{0} ; b_{i}, c_{i}$ - нечетные и четные координаты вектора $\mathbf{a}$.

Далее преобразуем уравнение ротатора. Подставляя выражение для фазы $\dot{\varphi}=\omega_{0}+\mu \Phi(\varphi, \psi, \xi, \theta, \eta)$ в первое уравнение (П.3.2), получаем

$$
\begin{array}{r}
\frac{\partial \Phi}{\partial \varphi}\left(\omega_{0}+\mu \Phi\right)+\frac{\partial \Phi}{\partial \psi} \omega_{0}+\sum_{j=1}^{m} \frac{\partial \Phi}{\partial \theta_{j}} \mu \Theta_{j}+\sum_{j=1}^{m} \frac{\partial \Phi}{\partial \eta_{j}} \mu \mathbf{T}_{j}+\dot{\xi}+ \\
+\delta\left(1+F_{1}\right)\left(\omega_{0}+\mu \Phi\right)+F_{2}=\gamma+\mathbf{b}^{T} \mathbf{x}+\mathbf{c}^{T} \mathbf{y}+f(\psi)+\mu F^{*}(\varphi, \mathbf{z}) .
\end{array}
$$

При известных требованиях для функции Ф из (П.3.3) определяем ее уравнение

$$
\frac{\partial \Phi}{\partial \varphi} \omega_{0}+\frac{\partial \Phi}{\partial \psi} \omega_{0}+\delta \omega_{0} F_{1}+F_{2}=\mathbf{b}^{T} \mathbf{x}+\mathbf{c}^{T} \mathbf{y}+f(\psi)
$$

и резонансную зону параметров $\gamma-\delta \omega_{0}=\mu \Delta$.

Решение уравнения (П.3.4) имеет вид:

$$
\begin{gathered}
\Phi=- \\
\delta \int F_{1}(\varphi) d \varphi-\frac{1}{\omega_{0}} \int F_{2}(\varphi) d \varphi+\frac{1}{\omega_{0}} \int f(\psi) d \varphi+ \\
+\frac{1}{\omega_{0}} \int \mathbf{b}^{T} \mathbf{x}(\varphi) d \varphi+\frac{1}{\omega_{0}} \int \mathbf{c}^{T} \mathbf{y}(\varphi) d \varphi+\xi,
\end{gathered}
$$

где $\quad \frac{1}{\omega_{0}} \int \mathbf{b}^{T} \mathbf{x}(\varphi) d \varphi=\frac{1}{\omega_{0}} \sum_{i=1}^{m} \frac{b_{i}}{n_{i}}\left(-\theta_{i} \cos n_{i} \varphi+\eta_{i} \sin n_{i} \varphi\right) \frac{1}{\omega_{0}} \int \mathbf{c}^{T} \mathbf{y}(\varphi) d \varphi=$ $=\sum_{i=1}^{m} c_{i}\left(\theta_{i} \sin n_{i} \varphi+\eta_{i} \cos n_{i} \varphi\right)$.

Выражение для функции $\Xi$ :

$$
\Xi=\Delta-\frac{\partial \Phi}{\partial \varphi} \Phi-\delta\left(1+F_{1}\right) \Phi-\sum_{j=1}^{m} \frac{\partial \Phi}{\partial \theta_{j}} \Theta_{j}-\sum_{j=1}^{m} \frac{\partial \Phi}{\partial \eta_{j}} T_{j}+F^{*}(\varphi, \mathbf{z}) .
$$

В итоге получаем эквивалентную (П.3.1) систему в стандартной форме: 


$$
\begin{aligned}
& \dot{\xi}=\mu \Xi, \\
& \dot{\theta}=\mu \Theta, \\
& \dot{\eta}=\mu \mathbf{T}, \\
& \dot{\chi}=\mu \Phi, \\
& \dot{\varphi}=\omega_{0}+\mu \Phi,
\end{aligned}
$$

где $\chi=\varphi-\psi-$ фазовая расстройка.

Рассмотрим систему произвольного числа связанных ротаторов, каждый из которых нагружен колебательной нагрузкой. Уравнения нагрузок могут иметь различные размерности и спектры частот. Будем предполагать, что выполнены упомянутые выше условия для каждой из них и их уравнения преобразованы к стандартной форме по указанному выше образцу. После всех процедур приходим к этапу преобразования системы вида

$$
\begin{aligned}
& I \ddot{\varphi}_{k}+\delta_{k}\left(1+F_{1 k}\left(\varphi_{k}\right)\right) \dot{\varphi}_{k}+F_{2 k}\left(\varphi_{k}\right)=\gamma_{k}+f_{k}(\psi)+ \\
& +\sum_{j=1}^{n} f_{k j}\left(\varphi_{j}\right)+\sum_{j=1}^{n} \beta_{k j} \dot{\varphi}_{j}+\mathbf{b}_{k}^{T} \mathbf{x}_{k}+\mathbf{c}_{k}^{T} \mathbf{y}_{k}+\mu F_{k}^{*}(\varphi, \mathbf{z}), \\
& \dot{\theta}_{k}=\mu \Theta_{k}, \\
& \dot{\eta}_{k}=\mu \mathbf{T}_{k}, \\
& \dot{\psi}=\omega_{0},
\end{aligned}
$$

где $\mathbf{b}_{k}^{T} \mathbf{x}_{k}=\sum_{i=1}^{m_{k}} b_{k i}\left(\theta_{k i} \sin n_{k i} \varphi+\eta_{k i} \cos n_{k i} \varphi\right), \mathbf{c}_{k}^{T} \mathbf{y}_{k}=\sum_{i=1}^{m_{k}} c_{k i}\left(\theta_{k i} \cos n_{k i} \varphi-\right.$ $\left.-\eta_{k i} \sin n_{k i} \varphi\right) n_{k i} \omega_{0}, \varphi-$ одна из фаз ротаторов.

Определяя форму уравнений для фаз в виде $\dot{\varphi}_{k}=\omega_{0}+$ $+\mu \Phi_{k}\left(\varphi, \psi, \theta_{k}, \eta_{k}, \xi_{k}\right)$ и подставляя эти выражения в уравнения ротаторов, получаем уравнения для функций $\Phi_{k}\left(\varphi, \psi, \theta_{k}, \eta_{k}, \xi_{k}\right)$, выражения для функций $\Xi_{k}$ и резонансные зоны параметров 


$$
\begin{aligned}
& \sum_{j=1}^{n} \frac{\partial \Phi_{k}}{\partial \varphi_{j}} \omega_{0}+\frac{\partial \Phi_{k}}{\partial \psi} \omega_{0}+\delta_{k} \omega_{0} F_{1 k}\left(\varphi_{k}\right)+F_{2 k}\left(\varphi_{k}\right)= \\
& =\sum_{j=1}^{n} f_{k j}\left(\varphi_{j}\right)+f_{k}(\psi)+\mathbf{b}_{k}^{T} \mathbf{x}_{k}+\mathbf{c}_{k}^{T} \mathbf{y}_{k}, \\
& \gamma_{k}-\left(\delta_{k}+\mathbf{a}_{k}^{T} \mathbf{B}_{k}^{-1} \mathbf{b}_{k}-\sum_{j=1}^{n} \beta_{k j}\right) \omega_{0}=\mu \Delta_{k}, k=\overline{1, n} .
\end{aligned}
$$

Интересующее нас решение уравнения (П.3.7) находится без труда:

$$
\begin{aligned}
& \Phi_{k}=\frac{1}{\omega_{0}} \sum_{j=1}^{n} \int f_{k j}\left(\varphi_{j}\right) d \varphi_{j}+\frac{1}{\omega_{0}} \int f_{k}(\psi) d \psi-\delta_{k} \int F_{1 k}\left(\varphi_{k}\right) d \varphi_{k}- \\
& -\frac{1}{\omega_{0}} \int F_{2 k}\left(\varphi_{k}\right) d \varphi_{k}+\frac{1}{\omega_{0}} \int \mathbf{b}_{k}^{T} \mathbf{x}_{k}(\varphi) d \varphi+\frac{1}{\omega_{0}} \int \mathbf{c}_{k}^{T} \mathbf{y}_{k}(\varphi) d \varphi+\xi_{k} .
\end{aligned}
$$

При этом имеем следующие выражения для функций $\Xi_{k}$ :

$$
\begin{aligned}
& \Xi_{k}=\Delta_{k}+\sum_{j=1}^{n}\left(\beta_{k j}-\frac{\partial \Phi_{k}}{\partial \varphi_{j}}\right) \Phi_{j}-\delta_{k} \Phi_{k}\left(1+F_{1 k}\right)- \\
& -\sum_{j=1}^{m} \frac{\partial \Phi_{k}}{\partial \theta_{k j}} \Theta_{k j}-\sum_{j=1}^{m} \frac{\partial \Phi_{k}}{\partial \eta_{k j}} T_{k j}+F_{k}^{*} .
\end{aligned}
$$

В итоге получаем эквивалентную уравнениям (П.3.6) систему в стандартной форме с быстровращающейся фазой:

$$
\begin{aligned}
& \dot{\xi}_{k}=\mu \Xi_{k}, \\
& \dot{\theta}_{k}=\mu \Theta_{k}, \\
& \dot{\eta}_{k}=\mu \mathbf{T}_{k}, \\
& \dot{\chi}_{k}=\mu\left(\Phi-\Phi_{k}\right), \\
& \dot{\varphi}=\omega_{0}+\mu \Phi,
\end{aligned}
$$

где $\chi_{k}=\varphi-\varphi_{k}-$ фазовые расстройки, $\varphi-$ одна из фаз ротаторов. 
Приложение II

\section{ВЫЧИСЛЕНИЕ СОБСТВЕННЫХ ЗНАЧЕНИЙ МАТРИЦ}

Рассмотрим матрицу $\mathbf{A}_{p}$ и определитель $\Delta_{p}$ вида

$$
\mathbf{A}_{p}=\left(\begin{array}{ccccccccc}
2 v+\alpha & -1 & 0 & 0 & & & & & \\
-1 & 2 v & -1 & 0 & & & & & \\
0 & -1 & 2 v & -1 & & & & 0 & \\
0 & 0 & -1 & 2 v & & & & & \\
& 0 & & & \ddots & & & 0 & \\
& & & & & 2 v & -1 & 0 & 0 \\
& & & & & -1 & 2 v & -1 & 0 \\
& & & & & 0 & -1 & 2 v & -1 \\
& & & & & 0 & 0 & -1 & 2 v+\beta
\end{array}\right),
$$

$$
\Delta_{p}=\left|\begin{array}{ccccccccc}
2 v & -1 & 0 & 0 & & & & & \\
-1 & 2 v & -1 & 0 & & & & & \\
0 & -1 & 2 v & -1 & & & & & \\
0 & 0 & -1 & 2 v & & & & & \\
& 0 & & \ddots & & 0 & & \\
& & & & & 2 v & -1 & 0 & 0 \\
& 0 & & 0 & -1 & 2 v & -1 & 0 \\
& & & & & 0 & -1 & 2 v & -1 \\
& & & & & 0 & 0 & -1 & 2 v
\end{array}\right| .
$$

Можно установить, что для главных миноров определителя имеет место рекуррентное уравнение вида [66]

$$
\Delta_{k}=2 v \Delta_{k-1}-\Delta_{k-2}, \quad k=\overline{1, p},
$$


с начальными условиями $\Delta_{-1}=0, \Delta_{0}=1$. В интересующих нас случаях $|v|<1$. При этом условии уравнение имеет следующее решение

$$
\Delta_{k}=\frac{\sin (k+1) \theta}{\sin \theta}, \theta=\arccos v .
$$

Рассмотрим многочлен $P(\boldsymbol{v})=\operatorname{det} \mathbf{A}_{n}$. Раскладывая определитель матрицы по первой, а затем по последней строке, получаем выражение искомого многочлена:

$$
P(v)=(2 v+\alpha)(2 v+\beta) \Delta_{p-2}-(4 v+\alpha+\beta) \Delta_{p-3}+\Delta_{p-4} .
$$

С учетом рекуррентного уравнения многочлен упрощается:

$$
P(v)=\Delta_{p}+(\alpha+\beta) \Delta_{p-1}+\alpha \beta \Delta_{p-2} .
$$

Если в этом уравнении положить, что $v=1-\lambda / 2$, то $P(v) \equiv P(\lambda)$ есть характеристический многочлен матрицы, а $P(\lambda)=0$ - ее характеристическое уравнение. Рассмотрим некоторые случаи значений параметров.

1) $\alpha=-1, \beta=1$. В этом случае

$$
P(v)=\Delta_{p}-\Delta_{p-2}=\frac{\sin (p+1) \theta-\sin (p-1) \theta}{\sin \theta}=2 \cos p \theta .
$$

Характеристическое уравнение имеет вид

$$
\cos p \theta=0 \text {. }
$$

Решая его, получаем собственные значения матрицы:

$$
\lambda_{j}=4 \sin ^{2} \frac{\pi+2 \pi j}{4 p}, j=\overline{0, p-1} .
$$

2) $\alpha=-1, \beta=0$. В этом случае получаем следующее характеристическое уравнение:

$$
P(v)=\Delta_{p}-\Delta_{p-1}=\frac{\cos (p+1 / 2) \theta}{\cos \theta / 2}=0 .
$$


Решая его, получим корни матрицы:

$$
\lambda_{j}=\frac{4 \sin ^{2}(\pi+2 \pi j)}{2(2 p+1)}, \quad j=\overline{0, p-1} .
$$

3) $\alpha=0, \beta=0$. В этом случае $P(v)=\Delta_{p}=\frac{\sin (p+1) \theta}{\sin \theta}$. Решая характеристическое уравнение, получаем корни:

$$
\lambda_{j}=\frac{4 \sin ^{2} \pi j}{2(p+1)}, \quad j=\overline{1, p} .
$$




\section{СПИСОК ЛИТЕРАТУРЫ}

1. Андронов А. А., Витт А. А., Хайкин С. Э. Теория колебаний. М.: Физматгиз, 1959.

2. Барбашин Е. А., Табуева В. А. Динамические системы с цилиндрическим фазовым пространством. - М.: Наука, 1969.

3. Josephson B. D. The discovery of tunnely supercurrents // Rev. Mod. Phys. 1974. Vol. 46. P. 252-254.

4. Barone A., Paterno G. Physics and Applications of the Josephson Effect. - New York: Wiley, 1982.

5. Лихарев К. К. Введение в динамику джозефсоновских переходов. - М.: Наука, 1985.

6. Горев А. А. Избранные труды по вопросам устойчивости электрических систем. - М.: Госэнергоиздат, 1960.

7. Блехман И. И. Синхронизация динамических систем. - М.: Наука, 1971.

8. Алифов А. А., Фролов К.В. Взаимодействие нелинейных колебательных систем с источниками энергии. - М.: Наука, 1985.

9. Adams M. L. Rotating machinery vibration: from analysis to troubleshooting. - Marcel Dekker, 2000.

10. Акимов В. Н., Белюстина Л. Н., Белых. В. Н. и др. Системы фазовой синхронизации / Под ред. В. В. Шахгильдяна и Л. Н. Белюстиной. - М.: Радио и связь, 1982.

11. Lindsey W. C. Synchronization systems in communication control. - New Jersey: Prentice-Hall, Inc., Englewood Cliffs, 1972.

12. Баутин Н. Н., Леонтович Е. А. Методы и приемы качественного исследования динамических систем на плоскости. - М.: Наука, 1990.

13. Van der Pol B. Forced oscillation in circuit with non-linear resistance // Philos. Mag. Ser. 7. 1927. Vol. 3, N 13. P. 65-80.

14. Крылов Н. М., Боголюбов Н. Н. Введение в нелинейную механику. - Киев: Изд-во АН УССР, 1937.

15. Hale J. K. Oscillations in nonlinear systems. - New York: McGrawHill, 1963.

16. Митропольский Ю. А., Лыкова О. Б. Интегральные многообразия в нелинейной механике. - М. Наука, 1973. 
17. Неймарк Ю. И. Метод точечных отображений в теории нелинейных колебаний. - М.: Наука, 1972.

18. Волосов В. М., Моргунов Б. И. Метод осреднения в теории нелинейных колебательных систем. - М.: Изд-во Моск. ун-та, 1971.

19. Андронов А. А., Леонтович Е. А., Гордон И. И., Майер А. Г. Качественная теория динамических систем на плоскости. - М.: Наука, 1966.

20. Magnus K. Schwingungen. - Stuttgart: Teubner, 1976.

21. Королев В. И. Фуфаев Н. А., Чеснокова Р. А. О корректности приближенного исследования качания ротора синхронной машины // Прикладная математика и механика. 1973. Т. 37, № 6. C. $1007-1014$.

22. McCumber D. E. Effect of AC impedance on DC voltagecurrent characteristics of superconductor weak-link junctions // J. of Appl. Phys. 1968. Vol. 39. P. 3113.

23. Afraimovich V. S., Shilnikov L. P. Invariant tori, their breakdown and stochastisity // Amer. Math. Soc. Transl. 1991. Vol. 149. P. 201-211.

24. Итоги науки и техники. Современные проблемы математики. Фундаментальные направления / Под ред. Р. В. Гамкрелидзе, Д. В. Аносова, В. И. Арнольда. Т. 5. - М.: ВИНИТИ, 1985.

25. Abramowitz M., Stegun I. A. Handbook of mathematical functions. - New York: Dover. Publications Inc., 1964.

26. Anishchenko V. S. Dynamical chaos basic concepts. - Leipzig: Teubner-Texte zur Physik, 1987.

27. Belykh V. N., Pedersen N. F., Soerensen O. H. Shunted Josephson junction model. Part 1. Autonomous case. Part 2. Nonautonomous case // Phys. Rev. B. 1977. Vol. 16, N 11. P. 4853-4871.

28. Биргер И. А., Пановко Я. Г. Прочность. Устойчивость. Колебания: Справочник в 3-х т. - М.: Машиностроение, 1968.

29. Белых В. Н., Некоркин В. И. Качественное исследование системы трех дифференциальных уравнений из теории фазовой синхронизации // Прикладная математика и механика. 1975. Т. 39, № 4. C. $642-649$.

30. Shilnikov L. P. Theory of bifurcations of dynamical systems with homoclinic Poincare curves // VII Int. Conf. for Nonlinear Oscillations. Bd. 12. - Berlin: Akad-Verlag, 1977. 
31. Shilnikov L. P., Turaev D. V. A new simple bifurcation of a periodic orbit of blue sky catastrophe type // Amer. Math. Soc. Transl. Ser. II «Methods of qualitative theory of differential equations and related topics». 2000. Vol. 200. P. 165-188.

32. Белых В. Н. Веричев Н. Н. О динамике взаимодействующих ротатора и осциллятора // Изв. вузов. Сер. Радиофизика. 1988. № 7.

33. Бутенин Н. В., Неймарк Ю. И., Фуфаев Н. А. Введение в теорию нелинейных колебаний. - М.: Наука, 1976.

34. Блехман И. И. Вибрации в технике. Т. 2. Колебания нелинейных механических систем. - М.: Машиностроение, 1979.

35. Веричев Н. Н., Веричев С. Н., Ерофеев В. И. Хаотическая динамика вибрационных механизмов с источниками энергии ограниченной мощности // Прикладная математика и механика. 2007. Т. 71, № 3. С. 439-450.

36. Веричев Н.Н. Исследование систем с джозефсоновскими контактами методом быстро вращающейся фазы // Радиотехника и электроника. 1986. Т. 31, № 11. С. 2267-2274.

37. Афраймович В. С., Быков В. В., Шильников Л. П. О притягивающих негрубых предельных множествах типа аттрактора Лоренца // Тр. Моск. матем. общ-ва. 1982. Т. 44. С. 180-212.

38. Feigenbaum M. J. Quantitative universaliti for a class nonlinear trasformations // J. Stat. Phys. 1978. Vol. 19, N 1. P. 25-52.

39. Минц Р. М. Исследование траекторий системы трех дифференциальных уравнений в бесконечности // Памяти А. А. Андронова. - М.: Изд-во АН СССР, 1955. С. 499-534.

40. Белых В. Н., Веричев Н. Н. О динамике взаимосвязанных ротаторов // Изв. вузов. Сер. Радиофизика. 1988. Т. XXXI, № 6. C. $688-697$.

41. Белых В. Н., Веричев Н. Н. О сложной динамике одной автономной системы с джозефсоновскими контактами // Радиотехника и электроника. 1987. Т. 31, № 1. С. 140-147.

42. Lorenz E. Deterministic nonperiodic flow // J. Atmos. Sci. 1963. Vol. 20. P. 130-141.

43. Sommerfeld A. // VDI. 1904. Vol. 18.

44. Калищук А. К. Элементарный способ изучения динамических свойств систем // ЖТФ. 1936. Вып. 9, № 8. С. 687-696. 
45. Мартышкин В. С. Установка для изучения динамических характеристик строительных материалов // Динамические свойства строительных материалов. - М.: Стройиздат, 1940.

46. Блехман И. И. Самосинхронизация вибраторов некоторых вибрационных машин // Инженерный сборник. 1953. Т. 16. С. 49-72.

47. Кононенко В. О. Колебательные системы с ограниченным возбуждением. - М.: Наука, 1964.

48. Филиппов А. П. Колебания деформируемых систем. - М.: Машиностроение, 1970.

49. Пат. РФ № 2456481 C2, МПК F04D15/00, F04D13/10. Подавление поперечных вибраций электроцентробежного насоса посредством модулирования скорости вращения двигателя / Н. Н. Веричев, Н. С. Веричев // Бюл. изобр. 20.07.2012.

50. Веричев Н. Н., Веричев С. Н., Ерофеев В. И. Гашение изгибных колебаний вращающегося вала // Вестник машиностроения. 2012. № 8. C. $26-30$.

51. Verichev N. N. Chaotic torsional vibration of imbalanced shaft driven by a limited power supply // J. of Sound and Vibration. 2012. Vol. 331, Iss. 2. P. 384-393.

52. Гапонов-Грехов А. В., Рабинович М. И., Старобинец И. М. // Письма в ЖЭТФ. 1982. Т. 39. С. 561.

53. Афраймович В. С., Рабинович М. И., Сбитнев В. И. // Письма в ЖТФ. 1984. Т. 6. С. 338.

54. Арансон И. С., Гапонов-Грехов А. В., Рабинович М. И., Старобинец И. М. // ЖЭТФ. 1986. Т. 90. С. 1707.

55. Анищенко В. С., Арансон И. С., Постнов Д. Э., Рабинович М. И. // ДАН СССР. 1986. Т. 286, № 5. С. 628.

56. Арансон И. В., Веричев Н. Н. Динамика квазипериодических волновых движений в однонаправленных цепочках генераторов // Изв. вузов. Сер. Радиофизика. 1988. Т. 31, № 1. С. 29-40.

57. Афраймович В. С., Некоркин В. И., Осипов Г. В., Шалфеев В. Д. Устойчивость, структуры и хаос в нелинейных сетях синхронизации / Под ред. А. В. Гапонова-Грехова и М. И. Рабиновича. Горький: ИПФ АН СССР, 1989.

58. Абарбанель Г. Д., Рабинович М. И., Селверстон А. и др. Синхронизация в нейронных ансамблях // УФН. 1996. Т. 166, № 4. C. $363-390$. 
59. Борисюк Г. Н., Борисюк Р. М., Казанович Я. Б., Селверстон А. и др. Моделирование динамики нейронной активности и обработка информации в мозге - итоги «десятилетия» // УФН. 2002. Т. 172, № 10. C. $1189-1214$.

60. Carpenter G. A. Neural network models for pattern recognition and associative memory // Neural Networks. 1989. Vol. 2. P. 243-257.

61. Kohonen T. Self-organizing maps. - Berlin: Shpringer, 1997.

62. Tan Z., Ali M. K. Pattern recognition in a neural network with chaos // Phys. Rev. E. 1998. Vol. 58. P. 36-49.

63. Веричев Н. Н. Устойчивость структур в неравновесных системах // Вычислительная механика сплошных сред. 2013. Т. 6, № 1. C. $23-33$.

64. Pecora L. M., Carroll T. L. Synchronization in chaotic systems // Phys. Rev. Lett. 1990. Vol. 64. P. 821.

65. Ланкастер П. Теория матриц. - М.: Наука, 1982.

66. Белых В. Н., Веричев Н. Н. Пространственно-однородные автоволновые процессы в системах с переносом и диффузией // Изв. вузов. Сер. Радиофизика. 1996. Т. 39, № 5. С. $588-596$.

67. Веричев Н. Н., Веричев С. Н., Ерофеев В. И. Кластерная динамика однородной цепочки диссипативно связанных ротаторов // Прикладная математика и механика. 2008. Т. 72, № 6. С. 882-897.

68. Афраймович В. С., Веричев Н. Н., Рабинович М. И. Стохастическая синхронизация колебаний в диссипативных системах // Изв. вузов. Сер. Радиофизика. 1986. Т. 29. С. 795.

69. Белых В. Н., Белых И. В., Веричев Н. Н. Регулярные и хаотические пространственно-однородные колебания в цепочке взаимосвязанных сверхпроводящих переходов // Изв. вузов. Сер. Радиофизика. 1997. Т. 15, № 7. С. $912-924$.

70. Белых И. В., Веричев Н. Н. Глобальная синхронизация и странные аттракторы в связанных маятниковых системах // Вестник ННГУ Нелинейная динамика и хаос. 1997. Т. 2. С. 93-102.

71. Josic K. Invariant manifolds and synchronization of coupled dynamical systems // Phys. Rev. Lett. 1998. Vol. 80. P. 3053-3056.

72. Kaneko K. Relevance of clustering to biological networks // Phys. D. 1994. Vol. 75. P. 55-73.

73. Kaneko K. Clustering, coding, switching, hierarchical ordering, and control in a network of chaotic elements // Phys. D. 1990. Vol. 41. P. $137-172$. 
74. Georgiou I. T., Bajaj A. K., Corless M. Invariant manifolds and chaotic vibrations in singularly perturbed nonlinear oscillators // Int. J. Eng. Sci. 1998. Vol. 36. P. 431-458.

75. Belykh V. N., Belykh I. V., Hasler M. Hierarchy and stability of partially synchronous oscillations of diffusively coupled dynamical systems // Phys. Rev. E. 2000. Vol. 62. P. 6332-6345.

76. Belykh V. N., Belykh I. V., Mosekilde E. Cluster synchronization modes in an ensemble of coupled chaotic oscillators // Phys. Rev. E. 2001. Vol. 63. P. 036216.

77. Belykh V., Belykh I., Hasler M., Nevidin K. Cluster synchronization in three-dimensional lattices of diffusively coupled oscillators // Int. J. Bifurc. Chaos. 2003. Vol. 13. P. 755-779.

78. Okuda K. Variety and generality of clustering in globally coupled oscillators // Phys. D. 1993. Vol. 63. P. 424-436.

79. Xie F., Hu G. // Phys. Rev. E. 1997. Vol. 55. P. 79.

80. Hasler M., Maistrenko Yu., Popovich O. // Phys. Rev. E. 1998. Vol. 58. P. 6843.

81. Zanette D. H., Mikailov A. S. // Phys. Rev. E. 1998. Vol. 57. P. 276.

82. Verichev N. N. C-oscillators and new outlook on cluster dynamics // J. of Phys. Conf. Ser. 23. 2005. P. 23-46.

83. Verichev N. N., Verichev S. N., Wiercigroch M. Physical interpretation and theory of existence of cluster structures in lattices of dynamical systems // Chaos, Solitons and Fractals. 2007. Vol. 34, Iss. 4. P. $1082-1104$.

84. Веричев Н. Н. Физика, существование и синтез кластерных структур связанных динамических систем // Нелинейный мир. 2009. T. 7, № 1. C. $28-45$.

85. Chua L. O., Komuro M., Matsumoto T. The double scroll family // IEEE Transaction on Circuits \& Systems. 1986. Vol. CAS-33, N 11. P. $1073-1118$.

86. Special Issue on Chua's Circuit // J. of Circuit, Systems, and Computers. 1993. Vol. 3, N 2.

87. Потапов А. А. Фракталы в радиофизике и радиолокации. М.: Логос, 2002.

88. Mandelbrot B. B. The fractal geometry of nature. - New York: Freeman, 1982. 
89. Потапов А. А., Гильмутдинов А. Х., Ушаков П. А. Фрактальные элементы и радиосистемы. Физические аспекты. - М.: Радиотехника, 2009.

90. Вонсовский С. В. Современная естественнонаучная картина мира. - Изд-во РХД, 2006.

91. Агол В. И., Богданов А. А., Гвоздев В. А. и др. Молекулярная биология. Структура и биосинтез нуклеиновых кислот / Под ред. А. С. Спирина. - М.: Высшая школа, 1990.

92. Osipov G. V., Sushchik M. M. Synchronized clusters and multistability in arrays of oscillators with different natural frequencies // Phys. Rev. E. 1998. Vol. 58. P. 7198.

93. Kanakov O. I, Osipov G. V., Chan C.-K., Kurths J. Cluster synchronization and spatio-temporal dynamics in networks of oscillatory and excitable Luo-Rudy cells // Chaos, Solitons and Fractals. 2007. Vol. 17. P. 015111.

94. Pecora L. M., Carroll T. L. // Phys. Rev. Lett. 1998. Vol. 80. P. 2109-2112.

95. Рабинович М. И., Трубецков Д. И. Введение в теорию колебаний и волн. - М: Наука, 1984.

96. Mosekilde E., Maistrenko Y., Postnov D. Chaotic synchronization: applications to living systems. - Singapure: World Scientific, 2002.

97. Wang W., Kiss I. Z., Hudson J. L. // Chaos. 2000. Vol. 10. P. 248.

98. Roy R., Thornburg K. S. // Phys. Rev. Lett. 2000. Vol. 72. P. 2009.

99. Cuomo K. M., Oppenheim A. V. // Phys. Rev. Lett. 1993. Vol. 71. P. 65.

100. Веричев Н. Н., Веричев С. Н., Ерофеев В. И. К-осцилляторы в однородном кольце диффузионно-связанных динамических систем: существование, устойчивость и синтез кластерных структур // Нелинейный мир. 2008. Т. 6, № 56. С. 398-423.

101. Гантмахер Ф. Р. Теория матриц. - М.: Наука, 1966.

102. Былов Ф., Виноград Р. Э., Гробман Д. М. Теория показателей Ляпунова и ее приложения к вопросам устойчивости. - М.: Наука, 1966.

103. Мышкис А. Д. Математика. Специальные курсы. - М.: Наука, 1971. 
Научное издание

Н. Н. Веричев, С. Н. Веричев, С. И. Герасимов, В. И. Ерофеев

\section{Хаос, синхронизация и структуры в динамике ротаторов}

Редактор, корректор Н. Ю. Зимакова

Компьютерная подготовка оригинала-макета С. Н. Фролова Дизайн обложки T. B. Андреева

Подписано в печать 08.12.2016 Формат 60×90/16 Печать офсетная Усл. печ. л. 16,7 Уч.-изд. л. 15,3 Тираж 200 экз. Зак. тип. 36-2016

Отпечатано в Издательско-полиграфическом комплексе ФГУП «РФЯЦ-ВНИИЭФ» 607188 , г. Саров Нижегородской обл., ул. Силкина, 23 